\title{
Problemas de Sincronismo em uma Família de Campos Vetoriais
}

\author{
Jerusa Mendonça Megale
}

\author{
TESE APRESENTADA \\ $\mathrm{AO}$ \\ Instituto de MATEMÁticA E EsTAtísticA \\ DA \\ UNIVERSIDADE DE SÃo PAUlo \\ PARA \\ OBTENÇÃO DO TÍTULO \\ DOUTORA EM CIÊNCIAS
}

Programa: Matemática Aplicada

Orientador: Prof. Dr. Manuel Valentim de Pera Garcia

O presente trabalho foi realizado com apoio da Coordenação de Aperfeiçoamento de Pessoal de Nível Superior - Brasil (CAPES) - Código de Financiamento 001

São Paulo, novembro de 2020 


\section{PROBLEMAS DE SINCRONISMO EM UMA FAMÍLIA DE CAMPOS VETORIAS}

Esta versão da tese contém as correções e alterações sugeridas pela Comissão Julgadora durante a defesa da versão original do trabalho, realizada em 10/12/2020. Uma cópia da versão original está disponível no Instituto de Matemática e Estatástica da Universidade de São Paulo.

Comissão Julgadora:

- Prof. Dr. Manuel Valentim de Pera Garcia - IME-USP

- Prof. Dr. Diego Cólon - EP-USP

- Prof. Dr. Fábio Armando Tal - IME-USP

- Prof. Dr. Ricardo dos Santos Freire Junior - IME-USP

- Prof. Dr. Ricardo Miranda Martins- UNICAMP-Externo 


\section{Agradecimentos}

Primeiro de tudo a Deus por me guiar e dar tranquilidade para atingir meus objetivos.

Aos meus pais e ao meu irmão que sempre me incentivaram e acreditaram que eu era capaz. Obrigada pela paciência e por estarem sempre presentes. Tudo que consegui só foi possível graças ao amor e dedicação que sempre tiveram por mim.

Ao Silas pela paciência e sempre me incentivar a correr atrás dos meus sonhos.

Ao meu orientador Prof. Manuel Valentim que me apoiou ao longo desses anos e nunca desistiu de mim. Obrigada por compartilhar seus conhecimentos comigo, pela compreensão e pelas palavras de incentivo quando tudo parecia perdido. Foi muito gratificante ser sua aluna.

A todos os professores do IME-USP que contribuíram para minha formação. Em especial, a Profa. Sônia Regina, pelo carinho, por sempre estar disponível para me ajudar e pela participação nos seminários. Suas orientações foram muito importantes para a realização deste trabalho.

Aos meus amigos Sueni, Pollyanna e Gregório que tornaram essa jornada mais divertida. Vocês são pessoas incríveis, foi maravilhoso conviver com vocês. Levo vocês no meu coração.

Ao meu colega de orientação Marcelo que me ajudou diversas vezes. Muito obrigada!

A todos os amigos que o doutorado me proporcionou e que torceram por mim. Gostaria de mencionar alguns: Larissa, Vanessa, Bruna, Ana Cecília, Raquel, Marisa, Daiana, João, Guilherme e Caio. Obrigada por todas as conversas e risadas.

Aos professores da banca pela leitura e considerações sobre este trabalho.

Finalmente, agradeço à CAPES pelo apoio financeiro. 


\section{Resumo}

Megale, J. M. Problemas de Sincronismo em uma Família de Campos Vetoriais. 2020. 111 f. Tese (Doutorado) - Instituto de Matemática e Estatística, Universidade de São Paulo, São Paulo, 2020 .

Esta tese é dedicada ao estudo de sistemas de forças quase-centrais tais que as funções $f$ e $g$ dependem apenas da variável $x$, isto é,

$$
\ddot{x}=-x f(x), \quad \ddot{y}=-y g(x), \quad f, g \in C^{\omega} .
$$

Fixada $f$ encontramos condições sobre $g$ para existir $\delta>0$ tal que, as soluções do sistema com condições inicias $x(0)=x_{0}, \dot{x}(0)=0, y(0)=y_{0}, \dot{y}(0)=\dot{y}_{0}$ sejam periódicas de menor período $\tau\left(x_{0}\right), \forall\left(x_{0}, y_{0}, \dot{y}_{0}\right) \neq 0$ com $0 \leq x_{0}<\delta$, em que $\tau\left(x_{0}\right)$ é o menor período da solução da primeira equação do sistema com condições iniciais $x(0)=x_{0}, \dot{x}(0)=0$. Denotamos esse fenômeno por $\tau\left(x_{0}\right)$-isocronismo.

Mostramos que a existência de $g$ satisfazendo essa propriedade é determinada pelo jato de ordem 2 de $f$ em 0.

Nosso resultado principal estabelece que para $f$ analítica real, definida e positiva numa vizinhança da origem, $I$, com $f(0)=1$ e satisfazendo $3 f^{\prime \prime}(0)>4 f^{\prime}(0)^{2}$, existem no máximo duas funções $g$, analíticas reais, definidas e positivas em $I$, tal que o sistema possui $\tau\left(x_{0}\right)$-isocronismo. Além disso, exibimos como será o resultado em série de potências das $g$ com essa propriedade, isso permite determinar explicitamente os possíveis jatos de ordem $k$ de $g$ em 0 . Esses ficam completamente determinados por $j^{(k)} g(0)$ e $j^{(k+1)} f(0)$.

Quando $3 f^{\prime \prime}(0)=4 f^{\prime}(0)^{2}$ não se tem o mesmo tipo de resultado, pois o sistema é degenerado. Neste caso, conseguimos determinar apenas os jatos pares de $g$ em 0 . O caso $f=g$ está contido nessa classe de sistemas.

Para $3 f^{\prime \prime}(0)<4 f^{\prime}(0)^{2}$ mostramos que não existe $g$ analítica real tal que o sistema possui $\tau\left(x_{0}\right)$ isocronismo.

Palavras-chave: equações de segunda ordem, campo de vetores, equações de Hill, sincronismo. 


\section{Abstract}

Megale, J.M Synchronism problems in a family of vector fields 2020. $111 \mathrm{f}$. Tese (Doutorado) - Instituto de Matemática e Estatística, Universidade de São Paulo, São Paulo, 2020.

This thesis is dedicated to the study of quasi-central force systems where the functions $f$ and $g$ depend only on the variable $x$, that is,

$$
\ddot{x}=-x f(x), \quad \ddot{y}=-y g(x), \quad f, g \in C^{\omega} .
$$

Fixing $f$, we find conditions on $g$ to exist $\delta>0$ such as the solutions of the system with initial conditions $x(0)=x_{0}, \dot{x}(0)=0, y(0)=y_{0}, \dot{y}(0)=\dot{y}_{0}$ are periodic with smallest period $\tau\left(x_{0}\right), \forall\left(x_{0}, y_{0}, \dot{y}_{0}\right) \neq(0,0,0)$ with $0 \leq x_{0}<\delta$, where $\tau\left(x_{0}\right)$ is the smallest period of the solution of the system's first equation with initial conditions $x(0)=x_{0}, \dot{x}=0$. We denote this phenomenon by $\tau\left(x_{0}\right)$-isochronism. We show that the existence of $g$ satisfying this property if defined by the order 2 jet of $f$ in 0 .

Our principal result establishes that for real analytic $f$, positive and defined in a neighborhood of the origin, $I$, with $f(0)=1$ and satisfying $3 f^{\prime \prime}(0)>4 f^{\prime}(0)^{2}$, there are at most two functions $g$, real analytic, positive and defined on $I$, such as the system has a $\tau\left(x_{0}\right)$-isochronism. Furthermore, we present how the result behaves in power series of $g$ carrying this property and this allows us to explicitly determine the possible order $k$ jets of $g(x)$ in 0 . These are completely determined by $j^{(k)} g(0)$ and $j^{(k+1)} f(0)$.

When $3 f^{\prime \prime}(0)=4 f^{\prime}(0)^{2}$ we do not have the same result since the system is degenerate. In this case, we could only determine the even jets of $g$ in 0 . This class of systems contains the case $f=g$.

For $3 f^{\prime \prime}(0)<4 f^{\prime}(0)^{2}$ we prove that there is no real analytic $g$ such that the system has $\tau\left(x_{0}\right)$ isochronism.

key-words: Second order equations, vector fields, Hill equations, synchronism; 


\section{Sumário}

1 Introdução $\quad 1$

1.1 Organização do Trabalho . . . . . . . . . . . . . . . . . . . . . . 4

2 Preliminares e fatos básicos $\quad 5$

2.1 Observações sobre equações de Hill . . . . . . . . . . . . . . . . . . . . 5

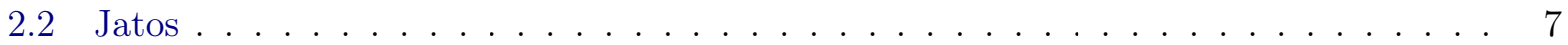

3 Alguns resultados de estabilidade para sistemas posicionais $\quad 9$

3.1 Dinâmica do sistema $\ddot{x}=-x f(x) \ldots \ldots \ldots \ldots \ldots$

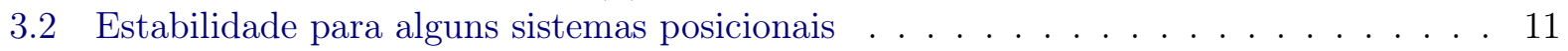

3.2.1 Sistemas do tipo: $X(x, y)=-x f(x), Y(x, y)=-y f(x) \ldots \ldots \ldots \ldots$

3.2.2 Sistemas do tipo: $X(x, y)=-x f(x), Y(x, y)=-y g(x) \ldots \ldots \ldots$

4 Condições computáveis para estabilidade da origem de sistemas posicionais e isocronismo $\quad 19$

4.1 Um método computacional para a estabilidade na origem . . . . . . . . . . . . 19

4.1 .1 As funções tempo de parada . . . . . . . . . . . . . . . . . . . . . 20

4.1 .2 Resultados de estabilidade . . . . . . . . . . . . . . . . . 21

4.2 Isocronismo para uma classe de sistemas hamiltonianos . . . . . . . . . . . . . . . 22

4.2 .1 Isocronismo no plano . . . . . . . . . . . . . . . . . . . . 23

4.2 .2 Resultados de Estabilidade . . . . . . . . . . . . . . . . . . . . 24

5 Condições necessárias e suficientes para o $\tau\left(x_{0}\right)$-isocronismo $\quad \mathbf{2 7}$

5.1 Resultados preliminares . . . . . . . . . . . . . . . . 27

5.1 .1 Introdução . . . . . . . . . . . . . . . . . . . . . . . . 27

5.2 Cálculo das derivadas de $\tau, t_{1}$ e $t_{2} \ldots \ldots \ldots \ldots \ldots \ldots \ldots \ldots \ldots \ldots \ldots \ldots$

5.2 .1 Equação das variações para $x$ e cálculo de $\tau^{(k)}(0) \ldots \ldots \ldots \ldots$. . . . . 32

5.2 .2 Equação das variações para $y_{1}$ e cálculo $t_{1}^{k}(0) \ldots \ldots$. . . . . . . . . . 37

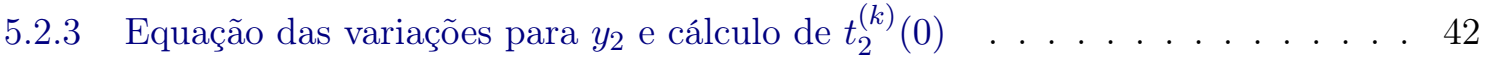

5.2 .4 Fórmulas das Derivadas de $\tau, t_{1}, t_{2} \ldots \ldots \ldots \ldots \ldots$

5.3 Propriedades das derivadas de $\tau, t_{1}, t_{2} \ldots \ldots \ldots \ldots \ldots \ldots$

6 Resultado Principal $\quad 53$

6.1 Resultados . . . . . . . . . . . . . . . . . . . . 54

6.1 .1 Demonstração do Lema $6.1 .1 \ldots \ldots \ldots$. . . . . . . . . . . . . 56

6.1 .2 Demonstração do Lema $6.1 .2 \ldots \ldots$. . . . . . . . . . . . . . . 59

7 Comentários e Aplicações $\quad 69$

7.1 Condições necessárias para o $\tau\left(x_{0}\right)$-isocronismo . . . . . . . . . . . . . . 69

7.2 Aplicações . . . . . . . . . . . . . . . . . . . . . . 72

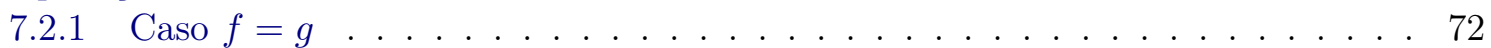

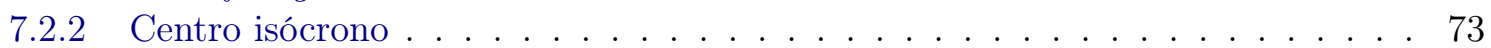

7.2 .3 Paridade da função $f(x)$ e $\tau\left(x_{0}\right)$-isocronismo $\ldots \ldots \ldots \ldots$. . . . . . . . 74 
7.3 Alguns resultados de estabilidade . . . . . . . . . . . . . . . . . 76

8 Considerações finais $\quad 81$

A Derivadas de $\tau, t_{1}$ e $t_{2}$ em zero até a quarta ordem

A.1 Algumas contas referentes ao cálculo das derivadas de $\tau$ em zero até a quarta ordem 83

A.2 Algumas contas referentes ao cálculo das derivadas de $t_{1}$ em zero até a quarta ordem 84

A.3 Algumas contas referentes ao cálculo das derivadas de $t_{2}$ em zero até a quarta ordem 86

B Demonstrações dos lemas auxiliares $\quad \mathbf{8 9}$

B.1 Demonstração do Lema $6.1 .3 \ldots \ldots \ldots$. . . . . . . . . . . . . . . . . 89

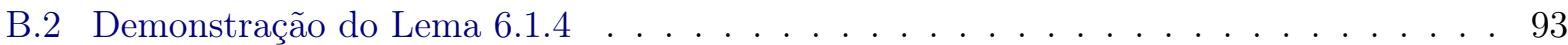

B.3 Demonstração do Lema $6.1 .5 \ldots \ldots \ldots$. . . . . . . . . . . . . . . . 97

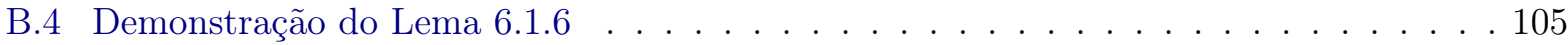

$\begin{array}{ll}\text { Referências Bibliográficas } & 111\end{array}$ 


\section{Capítulo 1}

\section{Introdução}

Considere o movimento de uma partícula de massa unitária no plano sujeito à ação de um campo de forças posicional, ou seja, a força depende apenas da posição da partícula.

Em linguagem matemática, de acordo com a linguagem Newtoniana, esse movimento é representado pela equação diferencial linear de segunda ordem

$$
\ddot{q}=F(q), \quad q=(x, y) \in \mathbb{R}^{2},
$$

em que $F: \mathbb{R}^{2} \mapsto \mathbb{R}^{2}$ é o campo de forças considerado.

Sejam $X(x, y)$ e $Y(x, y)$ as componentes da força imposta sobre a partícula, com $X, Y: \Omega=$ $\stackrel{\Omega}{\Omega} \mathbb{R}^{2} \mapsto \mathbb{R}, X, Y \in C^{1}$ e $X(0,0)=Y(0,0)=0$, as equações diferenciais são

$$
\left\{\begin{array}{l}
\ddot{x}=X(x, y) \\
\ddot{y}=Y(x, y), \quad(x, y) \in \Omega=\Omega \in \mathbb{R}^{2}, \quad 0 \in \Omega,
\end{array}\right.
$$

Definição 1.0.1. A força $F=(X ; Y)$ é dita conservativa se existe $\Pi: \Omega \mapsto \mathbb{R}, \Pi \in C^{2}$, tal que $\nabla \Pi,=-F$. Se não existe $\Pi$ satisfazendo essas condições a força é nãa-conservativa.

A teoria sobre a estabilidade de Liapunov para sistemas do tipo (1.1) sujeito à ação de forças atrativas, isto é, $F(q)$ do tipo $-f(q) q$, com $f(q)>0$, e conservativas é simples. Nestes casos, utilizando o Teorema de Dirichlet-Lagrange mostra-se que a estabilidade sempre ocorre.

Uma questão interessante é estudar a estabilidade segundo Liapunov dos equilíbrios de (1.1) quando a força $F=(X ; Y)$ é "quase central"no sentido descrito a seguir.

Definição 1.0.2. O sistema

$$
\left\{\begin{array}{l}
\ddot{x}=-x f(x, y) \\
\ddot{y}=-y g(x, y), \quad(x, y) \in \Omega=\Omega \in \mathbb{R}^{2}, \quad 0 \in \Omega,
\end{array}\right.
$$

com $f(x, y)>0$ e $g(x, y)>0$ para todo $(x, y) \in \Omega$, é chamado de sistema de forças quasecentral.

Note que o campo não é central, mas aponta para dentro das circunferências de centro na origem, no sentido que, se $q=(x, y)$, vale que o produto escalar $q . F(q)<0$, para todo $q \neq 0$. 
Nesta linha as principais referências são os trabalhos de A. Barone, M. O. Cesar, G. Zampieri e G. Gorni. Os estudos começaram no início dos anos 80, com um caso particularmente interesse que ocorre quando $f=g$, neste caso os sistemas são conhecidos como sistemas de forças-centrais:

$$
\left\{\begin{array}{l}
\ddot{x}=-x f(x, y) \\
\ddot{y}=-y f(x, y), \quad(x, y) \in \Omega=\Omega \in \mathbb{R}^{2}, \quad 0 \in \Omega
\end{array}\right.
$$

$\operatorname{com} f(x, y)>0$ para todo $(x, y) \in \Omega$,

Claro que a origem é o único ponto de equilíbrio dessa equação e o campo $F$ é radial e aponta para o centro (na direção da origem, em virtude do sinal negativo).

Se (1.4) é conservativo é simples ver que $f(x, y)$ é constante e há estabilidade.

O problema abordado por A.Barone e M.O.Cesar consistia em estudar a estabilidade da origem para estes sistemas no caso não conservativo e ficou conhecido informalmente, ao menos no IMEUSP, como "Problema da força que aponta". Durante anos A.Barone e M.O.Cesar estudaram esse problema, sem muito sucesso.

Em 2005, voltaram a estudá-lo e publicaram o artigo: [BNdOC05], no qual supondo $f$ analítica e a existência de uma integral primeira a mais do tipo energia para (1.4) foram obtidos alguns resultados de estabilidade para origem.

No final dos anos 80, em parceria com G. Zampieri, passaram a estudar a estabilidade da origem de sistemas não conservativos do tipo

$$
\ddot{x}=-x f(x), \quad \ddot{y}=-y f(x) .
$$

$\operatorname{com} f: U \mapsto \mathbb{R}, U$ aberto, $0 \in U, f(x)>0$ e $f$ contínua.

O objetivo era encontrar todos os casos estáveis, ou seja, encontrar todas as funções $f(x)$ para as quais a origem do sistema (1.5) é estável.

No artigo [Zam88], publicado em 1988, G.Zampiere construiu a classe de todos os casos estáveis, o que praticamente esgotou o problema da estabilidade para sistemas do tipo (1.5).

Assim, A.Barone, M.O.Cesar e G.Zampiere começaram a estudar a estabilidade da origem para sistemas (1.3) tais que as funções $f$ e $g$ dependem apenas da variável $x$, isto é,

$$
\ddot{x}=-x f(x), \quad \ddot{y}=-y g(x),
$$

com $f, g: U=\stackrel{\circ}{U} \subset \mathbb{R} \mapsto \mathbb{R}, 0 \in U, f, g \in C^{1}$ (no mínimo) e $f(x)>0, g(x)>0$.

A meta era encontrar condições sobre as funções $f$ e $g$ que implicassem na estabilidade da origem. Destaca-se o trabalho [BNdOCG02], em que A.Barone, M.O.Cesar e G. Gorni apresentam, para alguns casos, condições computáveis em termos das derivadas de $f(x)$ e $g(x)$ em 0 até a segunda ordem para a origem ser estável ou instável.

Além da estabilidade, os sistemas (1.6) foram estudados sobre outros aspectos. Dentre eles destaca-se o da coexistência de soluções periódicas.

Como $f(0)>0$, a origem $(x, \dot{x})=(0,0)$ é um centro para a equação $(1.6)_{1}$, logo as soluções são periódicas (para condições iniciais suficientemente perto de 0 ). Para $x_{0}>0$ fixado, considere a solução de $(1.6)_{1}$ com condições iniciais $\dot{x}(0)=x_{0}, \dot{x}(0)=0$. Seja $x\left(t ; x_{0}\right)$ essa solução, com menor período $\tau\left(x_{0}\right)$. 
Substituindo $x\left(t ; x_{0}\right)$ na segunda equação do sistema, obtemos a equação de Hill

$$
\ddot{y}=-y g\left(x\left(t ; x_{0}\right)\right)
$$

Dizemos que ocorre a coexistência de soluções periódicas se a solução de (??) com condições iniciais $y(0)=y_{0}$ e $\dot{y}(0)=\dot{y_{0}}$ é periódica para todo par $\left(y_{0}, \dot{y}_{0}\right)$.

Existem várias questões relacionadas a essa problema. Por exemplo:

Quais são as condições que as funções $f(x)$ e $g(x)$ devem satisfazer para que o sistema (1.6) apresente coexistência de soluções periódicas para cada $x_{0}$ suficientemente pequeno numa vizinhança de zero?

Surgiu-nos a seguinte questão: Dada $f(x)$ analítica, definida e positiva numa vizinhança de zero, $I$, existem funções $g(x)$ analíticas, definidas e positivas em $I$ tais que, para cada $x_{0}$ suficientemente pequeno numa vizinhança de zero, ocorra a coexistência de soluções periódicas e, além disso, todas as soluções sejam periódicas de menor período $\tau\left(x_{0}\right)$ ?

Se sim, é possível estabelecer condições sobre os $k$-jatos de $g(x)$ em zero que garantam que isso ocorra?

Nosso objetivo principal neste trabalho é responder essas perguntas.

O termo coexistência pode estar, neste tipo de problema, relacionado a outros aspectos, por isso nesta tese chamamos de $\tau\left(x_{0}\right)$ - isocronismo a propriedade: para cada $x_{0}$ suficientemente pequeno numa vizinhança de zero, ocorre a coexistência de soluções periódicas e, além disso, todas as soluções são periódicas de menor período $\tau\left(x_{0}\right)$.

Uma maneira equivalente de enunciar o problema é: Dada $f(x)$ satisfazendo as condições acima, existem funções $g(x)$ satisfazendo as mesmas condições tais que, para cada $x_{0}$ suficientemente pequeno numa vizinhança de zero, a solução de (1.7) com condições inicias $y(0)=y_{0}$ e $\dot{y}(0)=\dot{y}_{0}$ é periódica de menor período $\tau\left(x_{0}\right)$, para todo par $\left(y_{0}, \dot{y}_{0}\right)$ ?

Neste contexto, $\tau\left(x_{0}\right)$-isocronismo denota a seguinte situação: para cada $x_{0}$ suficientemente pequeno numa vizinhança de zero, a solução de (1.7) com condições inicias $y(0)=y_{0}$ e $\dot{y}(0)=\dot{y}_{0}$ é periódica de menor período $\tau\left(x_{0}\right)$, para todo par $\left(y_{0}, \dot{y}_{0}\right)$.

Mostraremos que se a função $f(x)$ satisfizer algumas condições sobre suas derivadas em zero, a resposta para as perguntas é positiva. Mais especificamente, mostraremos o Teorema A. Aqui e no resto do trabalho $j^{(k)} f(0)$ representa o polinômio de Taylor de ordem $k$ de $f$ em 0 (ou, em outro jargão, o $k$ - jato de $f$ em 0 , veja a Seção 2.2 para essa nomenclatura).

Teorema A: Considere o sistema (1.6), com $f(x)$ analítica real, definida e positiva numa vizinhança de $0 \in \mathbb{R}, I$, com $f(0)=1$.

i) Se $f^{\prime \prime}(0)>\frac{4}{3} f^{\prime}(0)^{2}$, então existem no máximo duas funções $g(x)$ analíticas reais, definidas e positivas em I, com $g(0)=1$, tal que o sistema possui $\tau\left(x_{0}\right)$-isocronismo. Uma delas tem

$$
g^{\prime}(0)=f^{\prime}(0)-\frac{1}{4} \sqrt{-24 f^{\prime}(0)^{2}+18 f^{\prime \prime}(0)}
$$

e a outra

$$
g^{\prime}(0)=f^{\prime}(0)+\frac{1}{4} \sqrt{-24 f^{\prime}(0)^{2}+18 f^{\prime \prime}(0)} .
$$

Além disso, se $g(x)$ é uma dessas funções e $k$ é um natural não nulo, $g^{(k+1)}(0)$ fica completa- 
mente determinado por $j^{(k)} g(0)$ e $j^{(k+1)} f(0)$.

ii) Se $f^{\prime \prime}(0)<\frac{4}{3} f^{\prime}(0)^{2}$, não existe $g(x)$ analítica real, definida e positiva em I, tal que o sistema possui $\tau\left(x_{0}\right)$-isocronismo.

iii) Se $f^{\prime \prime}(0)=\frac{4}{3} f^{\prime}(0)^{2}$ e g é analítica real, definida e positiva em $I$, tal que o sistema possui $\tau\left(x_{0}\right)$-isocronismo e $k$ natural impar não nulo, $g^{(k+1)}(0)$ fica completamente determinado por $j^{(k)} g(0)$ e $j^{(k+1)} f(0)$.

Também obtemos resultados para o caso de (1.6) ter $\tau\left(x_{0}\right)$-isocronismo e $f$ e $g$ serem de classe $C^{k}, k \geq 2$. Nesta situação determinamos, a partir de $j^{(k)} f(0)$ dado quais os possíveis $j^{(k)} g(0)$ para os quais existe $\tau\left(x_{0}\right)$-isocronismo (no Capítulo 7 exibi-se explicitamente essas possibilidades para o caso $k=4)$.

\subsection{Organização do Trabalho}

O texto está organizado da seguinte maneira. No Capítulo 2 faremos a revisão de alguns aspectos da Teoria de Equações de Hill que estarão presentes ao longo do texto.

Iniciaremos o Capítulo 3 com algumas considerações sobre a dinâmica do sistema planar $\ddot{x}=-x f(x)$, apresentaremos a função período $\tau\left(x_{0}\right)$ e suas principais propriedades.

A seguir, iremos enunciar os principais resultados de estabilidade obtidos por A. Barone, G. Zampieri e M .O. Cesar para sistemas do tipo (1.5) e (1.6). Mostraremos como nosso problema está relacionado com esses resultados de estabilidade.

Dedicaremos o Capítulo 4 à exposição dos artigos [BNdOCG02] e [Zam88]. O primeiro é de extrema importância para nós. A partir dele obtivemos ferramentas importantes para construção dos nossos resultados. Destacam-se as definições e propriedades das funções $t_{1}$ e $t_{2}$ e um método que permite calcular as derivadas dessas funções em zero.

O segundo apresenta resultados interessantes sobre isocronismo no plano, o que motivou algumas questões que são tratadas no texto.

No Capítulo 5 estão os nossos primeiros resultados. Demonstraremos o Teorema 5.1.2, que fornece condições necessárias e/ou suficientes, em termos das derivadas de ordem $k$ das funções $\tau, t_{1} \mathrm{e}$ $t_{2}$ em zero, para a ocorrência de $\tau\left(x_{0}\right)$-isocronismo em sistemas do tipo (1.6). Além disso, obteremos expressões para $\tau^{(k)}(0), t_{1}^{(k)}(0)$ e $t_{2}^{(k)}(0)$ e provaremos algumas propriedades dessas derivadas. Parte dos cálculos desse capítulo estão no Apêndice A.

No Capítulo 6, utilizando os resultados obtidos no capítulo anterior, demonstraremos o Teorema A supramencionado. Os cálculos com todos os detalhes estão no Apêndice B.

Para finalizar, no Capítulo 7 apresentaremos algumas aplicações interessantes do Teorema 5.1.2 e mostraremos como os resultados obtidos nestas aplicações estão relacionados com resultados já conhecidos de estabilidade. 


\section{Capítulo 2}

\section{Preliminares e fatos básicos}

Neste capítulo iremos relembrar alguns resultados básicos da Teoria de Equações de Hill , que utilizaremos ao longo do texto. Para mais informações sobre o assunto sugerimos a leitura de [MS66]. Apresentaremos também uma breve definição de jato de uma função na origem. Para uma visão mais aprofundada do assunto recomendamos a leitura de [Sai11].

No decorrer da tese utilizaremos vários resultados de Análise e Teoria de Equações Diferenciais Lineares. Dentre eles destacamos: o Teorema da depêndencia contínua das soluções com respeito às condições iniciais e o Método da Variação dos Parâmetros para resolução de sistemas lineares. Não iremos enunciar esses resultados. Como leitura complementar sugerimos [MSR04].

\subsection{Observações sobre equações de Hill}

Como é destacado no clássico texto de Magnus e Wilker [MS66], o termo "equação de Hill" é uma maneira conveniente de denominar equações diferenciais lineares de segunda ordem homogêneas com coeficientes periódicos.

Assim, "equação de Hill"denotaria uma equação

$$
\ddot{z}+a(t) \dot{z}+b(t) z=0
$$

em que $a(t)$ e $b(t)$ são funções periódicas de menor período $T>0$.

É bem conhecido que, no caso de $a(t)$ ser $C^{1}$, a mudança de variáveis

$$
y=\exp \left(\frac{A(t)}{2}\right) z
$$

em que $A(t)$ é uma primitiva de $a(t)$, transforma (2.1) em uma equação do tipo

$$
\ddot{y}+Q(t) y=0
$$

$\operatorname{com} Q(t)=b(t)-\frac{a(t)^{2}}{4}-\frac{\dot{a}(t)}{2}$.

Portanto, se $a(t)$ e $b(t)$ forem periódicas de menor período $T>0$, então $Q(t)$ também é periódica de período $T$, embora aqui nada garante que este é o menor período desta função (pode até ser constante).

Essa considerações justificam, apesar da ressalva feita sobre o menor período, a tendência atual 
em usar o termo "Equação de Hill" apenas para denotar equações do tipo (2.2).

Neste trabalho seguimos essa convenção da literatura atual.

Uma questão importante da Teoria de Equações de Hill é determinar a existência de soluções periódicas.

O valor do período da solução (que é um múltiplo do período $T$ dos coeficientes da Equação de Hill) é fundamental para caracterização das soluções periódicas. Na verdade, somente as soluções de período $T$ ou $2 T$ são interessantes.

A equação (2.2), com as condições acima, tem duas soluções continuamente diferenciáveis $y_{1}(t)$ e $y_{2}(t)$ determinadas pelas condições iniciais

$$
y_{1}(0)=1, \dot{y}_{1}(0)=0, y_{2}(0)=0, \dot{y}_{2}(0)=1 \text {. }
$$

Essas soluções são conhecidas como soluções normalizadas. E pela propriedade do Wronskiano

$$
y_{1}(t) \dot{y}_{2}(t)-y_{2}(t) \dot{y}_{1}(t)=1, \forall t
$$

É válido o seguinte teste de estabilidade:

Teorema 2.1.1. As soluções de (2.2) são estáveis se e somente se $\dot{y}_{1}(T)+y_{2}(T)$ é real e vale

$$
\left|\dot{y}_{1}(T)+y_{2}(T)\right|<2
$$

ou

$$
y_{1}(T)+\dot{y}_{2}(T)= \pm 2 \quad \text { e } \quad y_{2}(T)=\dot{y}_{1}(T)=0 .
$$

Se em (2.2) a função $Q(t)$ é par é possível estabelecer relações entre os valores de $y_{1}, \dot{y}_{1}, y_{2}$ e $\dot{y}_{2}$ em $T / 2$ e $T$, as quais nos fornecem mais informações sobre as soluções periódicas de período $T$ e $2 T$.

Teorema 2.1.2. Sejam $y_{1}(t)$ e $y_{2}(t)$ as soluções normalizadas de (2.2), com $Q(t)$ par. As seguintes relações ocorrem:

1. $y_{1}(T)=2 y_{1}(T / 2) \dot{y}_{2}(T / 2)-1=2 \dot{y}_{1}(T / 2) y_{2}(T / 2)+1$;

2. $y_{2}(T)=2 y_{2}(T / 2) \dot{y}_{2}(T / 2)$;

3. $\dot{y}_{1}(T)=2 y_{1}(T / 2) \dot{y}_{1}(T / 2)$;

4. $\dot{y}_{2}(T)=y_{1}(T)$.

Em todos os casos, $y_{1}(t)=y_{1}(-t)$, isto é, $y_{1}(t)$ é uma função par. Similarmente, $y_{2}(t)=-y_{2}(-t), y_{2}(t)$ é uma função impar em relação a $t$.

Sempre que existir uma solução não trivial de perído $T$ ou $2 T$, também existirá uma solução periódica não trivial que é par ou ímpar. Portanto, estas soluções periódicas são necessariamente múltiplas de uma das soluções normalizadas $y_{1}(t)$ ou $y_{2}(t)$, a menos que todas as soluções sejam periódicas (com período $T$ ou $2 T$ ).

O resultado a seguir exibe condições necessárias e suficientes para a existência de soluções periódicas de (2.2). 
Teorema 2.1.3. Supondo que as condições do Teorema 2.1 .2 são satisfeitas, se existe solução periódica não trivial de (2.2), então também existe uma solução não trivial que é par ou ímpar (podem existir ambas), de período $T$ ou $2 T$, e além disso, essa solução é:

1. par e de período $T$ se e semente se $\dot{y}_{1}(T / 2)=0$;

2. impar e de periodo $T$ se e somente se $y_{2}(T / 2)=0$;

3. par e de periodo $2 T$ se e somente se $y_{2}(T / 2)=0$;

4. impar e de periodo $2 T$ se e somente se $\dot{y}_{2}(t / 2)=0$.

Soluções periódicas pares ou ímpares de perído $T$ ou $2 T$ são necessariamente múltiplas das soluções normalizadas $y_{1}(t)$ ou $y_{2}(t)$.

\subsection{Jatos}

Nesta tese consideraremos funções diferenciáveis de abertos de $\mathbb{R}^{n}$ em $\mathbb{R}^{p}$ e neste contexto a noção de jato mostra-se uma ferramenta conveniente.

Denote por $J_{k}(n, p)$ o espaço vetorial real dos polinômios $g: \mathbb{R}^{n} \mapsto \mathbb{R}^{p} \operatorname{com} g(0)=0$.

Considere $U$ um subconjunto aberto de $\mathbb{R}^{n}$ e $f: U \mapsto \mathbb{R}^{p}$ de classe $C^{\infty}$.

Para $a \in U$ denote por $U_{a}=U-a=\left\{x \in \mathbb{R}^{n}: x+a \in U\right\}$ e por $j^{k} f(a)$ o polinômio de Taylor de ordem $k$ na origem de $h_{a}(x)=f(x+a)-f(a), x \in U_{a}$.

Como $h_{a}(0)=0$, tem-se que $j^{k} f(a) \in J_{k}(n, a)$.

Definição 2.2.1. Nestas condições, a função $j^{k} f: U \mapsto J^{k}(n, p)$ que a cada a associa $j^{k} f(a)$ chama-se jato $k$ de $f$ e o polinômio $j^{k} f(a)$ é denominado jato $k$ de $f$ em a (ou $k$-jato de $f$ em a).

Neste trabalho estamos interessados nas funções $f: I \mapsto \mathbb{R}$, em que $I$ é um intervalo aberto que contém o zero, e mais especial no seu $k$-jato que é $j^{k} f(a)=f^{\prime}(a) x+\frac{f^{\prime \prime}(a)}{2 !} x^{2}+\ldots \frac{f^{(k)}(a)}{k !} x^{k}$.

Devido ao nosso problema dar especial destaque ao $k$-jato de $f$ na origem, vai-se neste trabalho empregar como sinônimos esse jato $k$ e o próprio polinômio de Taylor de ordem $k$ de $f$ em zero (de fato esse último polinômio é, neste jargão, $\left.f(0)+j^{k} f(0)\right)$. 
PRELIMINARES E FATOS BÁSICOS 


\section{Capítulo 3}

\section{Alguns resultados de estabilidade para sistemas posicionais}

\subsection{Dinâmica do sistema $\ddot{x}=-x f(x)$}

Nesta seção faremos algumas considerações sobre a dinâmica do sistema planar $\ddot{x}=-x f(x)$. As principais referências são [Zam88] e [Zam11].

Apresentaremos a função período do sistema e suas propriedades. Como veremos ao longo do texto, a classe de diferenciabilidade dessa função será de grande importância para obtenção dos nossos resultados.

Para mais informações sobre a função período $\tau\left(x_{0}\right)$ e suas propriedades sugerimos a leitura do trabalho [dS02].

Considere o sistema

$$
\ddot{x}=-x f(x), \quad f: U \mapsto \mathbb{R}, \quad 0 \in U=\stackrel{\circ}{U} \subset \mathbb{R}, \quad f(x)>0
$$

com $f$ contínua, o que garante a unicidade de soluções.

Este sistema admite a integral primeira da energia

$$
E=\frac{\dot{x}^{2}}{2}+V(x), \quad V(x)=\int_{0}^{x} \sigma f(\sigma) d \sigma
$$

A energia potencial $V$ é de classe $C^{1} \mathrm{e}$

$$
V^{\prime \prime}(0)=\lim _{x \rightarrow 0} \frac{V^{\prime \prime}(x)}{x}=f(0)>0
$$

Como consequência a origem $x=0, \dot{x}=0$ é um centro para (3.1), isto é, existe uma vizinhança aberta $\Omega \subset \mathbb{R}^{2}$ da origem, invariante por (3.1), tal que todas as soluções de (3.1) com condições iniciais em $\Omega \backslash\{0,0\}$ são periódicas.

Além disso, cada uma dessas soluções corta a semi reta $(x, 0), x>0$ em um único ponto.

Assim, existe $\bar{x}_{0}$ tal que, o retrato de fase de (3.1) em $\Omega$ fica determinado pelas soluções $x(t)$ desta equação com condições iniciais $x(0)=x_{0}, \dot{x}(0)=0$, com $0<x_{0}<\bar{x}_{0}$ e, ademais, estas soluções são periódicas, p ortanto definidas em $\mathbb{R}$.

Denotaremos esta solução por $x\left(t, x_{0}\right)$. 
Note que, como $\tilde{x}(t)=x\left(-t, x_{0}\right)$ também é solução de $(3.1)$ e $\tilde{x}(0)=x_{0}, \dot{\tilde{x}}_{0}=0$, tem-se que, $\tilde{x}(t)=x(t)$ para todo $t$, ou seja, $x\left(t, x_{0}\right)$ é par.

Denotaremos por $\tau\left(x_{0}\right)$ o menor período de $t \mapsto x\left(t ; x_{0}\right)$, ou seja, se $0<\rho<\tau\left(x_{0}\right)$, então existe ao menos um intervalo real $I$ tal que $x\left(t+\rho ; x_{0}\right) \neq x\left(t ; x_{0}\right), \quad \forall t \in I$.

A partir de agora, sempre que dizermos que a solução é $\tau\left(x_{0}\right)$-periódica estamos admitindo que $\tau\left(x_{0}\right)$ é o menor período da solução.

Considere a função

$$
\bar{u}: x \mapsto \operatorname{sgn}(x) \sqrt{2 V(x)}, \quad \text { então } \quad \bar{u}(x)^{2}=2 V(x) .
$$

em que $\operatorname{sgn}(x)$ é o sinal de $x$.

Como

$$
\lim _{x \rightarrow 0} \frac{2 V(x)}{x^{2}}=f(0), \quad \text { então } \quad \bar{u}^{\prime}(0)=[f(0)]^{1 / 2}>0 .
$$

Além disso,

$$
\bar{u}^{\prime}(x)=\frac{x f(x)}{\bar{u}(x)} \mapsto \bar{u}^{\prime}(0)>0 \quad \text { quando } \quad x \mapsto 0 .
$$

Portanto, existe um intervalo maximal $J$ aberto, com $0 \in J$, tal que $u$ definida por $u=\bar{u} \mid J$ é um difeomorfismo de classe $C^{1}$ sobre um intervalo simétrico $I=u(J)$.

Definindo $h(x)=u^{-1}(-u(x))$, temos que $h$ também é um difeomorfismo:

$$
\begin{gathered}
h \in \operatorname{Diff}^{1}(J, J), \quad h(h(x))=x, \quad h(0)=0, \quad h^{\prime}(0)=-1 . \\
V(h(x))=V(x), \quad \operatorname{sgn}(h(x))=-\operatorname{sgn}(x) .
\end{gathered}
$$

A função $h$ é chamada de involução associada a $V$. Como $h^{-1}=h$, gráfico de $h$ é simétrico com respeito à diagonal dos quadrantes ímpares, ou seja, $(x, h(x))$ tem $(h(x), x)$ como ponto simétrico e coincide com o ponto $(h(x), h(h(x))$ no gráfico.

Considere a solução periódica que intersecta o eixo $x$ no ponto $x_{0} \in J$. Como $x_{0}>0$ então $h\left(x_{0}\right)<0$ é outra intersecção. Pela conservação de energia, temos que o período dessa solução é

$$
\tau\left(x_{0}\right)=2 \int_{h\left(x_{0}\right)}^{x_{0}} \frac{d x}{\sqrt{2\left(V\left(x_{0}\right)-V(x)\right)}} .
$$

A função $\tau\left(x_{0}\right)$ é tal que $\tau\left(x_{0}\right) \mapsto 2 \pi(f(0))^{1 / 2}$ quando $x_{0} \mapsto 0^{+}$.

De fato, considere (3.5). Se $y_{0} \in I, y_{0}>0, x_{0}=u^{-1}\left(y_{0}\right)$, então $h\left(x_{0}\right)=u^{-1}\left(-y_{0}\right)$, assim

$$
\begin{aligned}
T\left(y_{0}\right) & :=2 \int_{u^{-1}\left(-y_{0}\right)}^{u^{-1}\left(y_{0}\right)} \frac{d x}{\sqrt{2\left(V\left(x_{0}\right)-V(x)\right)}}=2 \int_{-y_{0}}^{y_{0}} \frac{\left(u^{-1}\right)^{\prime}(r) d r}{\sqrt{y_{0}^{2}-r^{2}}} \\
& =2 \int_{0}^{y_{0}} \frac{\left(\left(u^{-1}\right)^{\prime}(r)+\left(u^{-1}\right)^{\prime}(-r)\right) d r}{\sqrt{y_{0}^{2}-r^{2}}} .
\end{aligned}
$$

Fazendo a mudança de variável $s=\arcsin \left(\frac{r}{y_{0}}\right)$ para $y_{0} \in I, y_{0}>0$,

$$
T\left(y_{0}\right)=2 \int_{0}^{\frac{\pi}{2}}\left(\left(u^{-1}\right)^{\prime}\left(y_{0} \sin (s)\right)+\left(u^{-1}\right)^{\prime}\left(-y_{0} \sin (s)\right) d s .\right.
$$


Então,

$$
\lim _{x_{0} \mapsto 0_{+}} T\left(y_{0}\right)=2 \pi\left(u^{-1}\right)^{\prime}(0)=\frac{2 \pi}{\sqrt{V^{\prime \prime}(0)}}=\frac{2 \pi}{\sqrt{f(0)}} .
$$

Da fórmula (3.6), para $x_{0} \in J, x_{0}>0$, temos

$$
\tau\left(x_{0}\right)=2 \int_{0}^{\frac{\pi}{2}}\left(\left(u^{-1}\right)^{\prime}\left(u\left(x_{0}\right) \sin (s)\right)+\left(u^{-1}\right)^{\prime}\left(u\left(x_{0}\right) \sin (s)\right) d s .\right.
$$

Proposição 3.1.1. O período $\tau\left(x_{0}\right)$ de $t \mapsto x\left(t ; x_{0}\right)$ é uma função $C^{1}$ em $\left\{x_{0} \in J: x_{0}>0\right\}$.

Demonstração. Para $x_{0} \in J, x_{0}>0$, temos

$$
\tau\left(x_{0}\right)=2 \int_{0}^{\frac{\pi}{2}}\left(\left(u^{-1}\right)^{\prime}\left(u\left(x_{0}\right) \sin (s)\right)+\left(u^{-1}\right)^{\prime}\left(u\left(x_{0}\right) \sin (s)\right) d s .\right.
$$

A função $u$ é um $C^{1}$ difeomorfismo. Como $f \in C^{1}$, temos que $u(x)=\sqrt{V(x)}$, para $x>0$ é de classe $C^{2}$, bem como $V=f^{\prime}$ e a inversa $u^{-1}(y)$ para $y>0$. Isto prova o resultado.

A Proposição 3.1.1 nos fornece informações sobre a classe de diferenciabilidade da função período. Nesse caso, temos que $f$ é de classe $C^{1}$ o que implica $\tau\left(x_{0}\right)$ de classe $C^{1}$

É conhecido que a funções $f$ e $\tau\left(x_{0}\right)$ possuem a mesma classe de diferenciabilidade, ou seja, se $f$ e de classe $C^{r}$ então $\tau\left(x_{0}\right)$ também o é. Em particular, se $f$ é de classe $C^{\infty}$ a função $\tau\left(x_{0}\right)$ também é $C^{\infty}$, o que garante a existência das derivadas de $\tau\left(x_{0}\right)$ de todas as ordens.

No caso da função $f$ ser analítica o mesmo resultado é válido. Para prova desse resultado, sugerimos a leitura de [dS02].

\subsection{Estabilidade para alguns sistemas posicionais}

$\mathrm{Na}$ literatura, os principais resultados de estabilidade da origem para sistemas de forças quasicentrais são recorrentes dos trabalhos de A. Barone, M.O Cesar, G.Zampieri e G.Gorni.

Nesta seção apresentaremos alguns desses trabalhos. Aqui, restringimo-nos a enunciar os principais resultados obtidos e abordar algumas ideias envolvidas em suas demonstrações.

No Capítulo 7, utilizando os nossos teoremas, obteremos uma prova diferente de alguns desses resultados.

\subsubsection{Sistemas do tipo: $X(x, y)=-x f(x), Y(x, y)=-y f(x)$}

As referências para o estudo de sistemas desse tipo são os artigos [BNZ86],[BNdOC88] e [Zam88]. O objetivo principal desses é encontrar as funções $f(x)$ tais que a origem do sistema é estável.

Considere o sistema

$$
\ddot{x}=-x f(x), \quad \ddot{y}=-y f(x),
$$

$f: U=\stackrel{\circ}{U} \rightarrow \mathbb{R}, 0 \in U$ e $f$ contínua, o que garante a existência, unicidade e dependência contínua de soluções.

Note que se $f(0) \leq 0$, então a origem é instável. Para ver isto é suficiente considerar os movimentos ao longo do eixo $y$. Portanto, vamos assumir $f(0)>0$.

O sistema admite duas integrais primeiras. Uma delas é a integral da energia de $(3.7)_{1}$ e a outra é a integral das áreas, dadas, respectivamente, por 


$$
E(x, \dot{x})=\frac{\dot{x}^{2}}{2}+V(x), \quad \text { com } \quad V(x)=\int_{0}^{x} \sigma f(\sigma) d \sigma
$$

$\mathrm{e}$

$$
L(x, y, \dot{x}, \dot{y})=\dot{y} x-\dot{x} y=c .
$$

Como vimos na Seção 3.1, a origem no plano $x, \dot{x}$ é um centro, ou seja, todas as soluções, numa vizinhança da origem no plano $x, \dot{x}$, são periódicas e possuem a origem em seu interior.

Considere a solução $x\left(t ; x_{0} ; \dot{x}_{0}\right)$, satisfazendo $x(0)=x_{0}>0$ e $\dot{x}(0)=0$. Seja $T\left(x_{0}\right)$ o menor período dessa solução. Por simplicidade, denotaremos $T\left(x_{0}\right)$ por $T$.

Substituindo $x\left(t ; x_{0} ; 0\right)$ em $(3.7)_{1}$, obtemos a equação de Hill com coeficientes de período $T$ :

$$
\ddot{y}=-y f\left(x\left(t ; x_{0} ; 0\right)\right) \text {. }
$$

Da Teoria de Floquet (veja [MS66]) o sistema possui duas soluções normalizadas $y_{1}$ e $y_{2}$, satisfazendo as condições iniciais (2.3).

Note que $x\left(t, x_{0}, 0\right)=x\left(-t, x_{0}, 0\right), \operatorname{logo} y_{1}: t \mapsto x\left(t, x_{0}, 0\right) / x_{0}$ é a solução par de $(3.8)$ e

$$
y_{1}(0)=1, \quad \dot{y}_{1}(0)=0, \quad y_{1}(t+T)=y_{1}(t), \quad y_{1}(t)=y_{1}(-t) .
$$

Da integral de energia do sistema, segue que, se $h(x)$ é a função definida em na Seção 3.1, então

$$
t_{1}=\min \left\{t \in \mathbb{R}_{+}: y_{1}=0\right\} \quad \text { e } \quad h\left(x_{0}\right)=\max \left\{x \in \mathbb{R}_{-}: V(x)=V\left(x_{0}\right)\right\}
$$

e

$$
E=V\left(x_{0}\right), \quad y_{1}\left( \pm t_{1}\right)=0, \quad y_{1}\left( \pm t_{1}\right)=\mp \sqrt{2 V\left(x_{0}\right)}, \quad y_{1}(T / 2)=h\left(x_{0}\right) / x_{0}, \quad y_{1}(T / 2)=0,
$$

Consideremos agora a solução ímpar do sistema, $y_{2}(t)$. Da integral das áreas (que ao longo de $y_{2}(t)$ vale $x_{0}$, temos

$$
y_{2}\left( \pm t_{1}+n T\right)= \pm\left(2 V\left(x_{0}\right)\right)^{-1 / 2}, \quad \dot{y}_{2}(T / 2+n T)=x_{0} / h\left(x_{0}\right), \quad \dot{y}_{2}(n T)=1
$$

para cada $n \in \mathbb{Z}$.

Seja $t \mapsto \phi(t)$ solução de (3.8), temos que

$$
\phi(t)=\phi(0) y_{1}(t)+\dot{\phi}(0) y_{2}(t)
$$

A função $y_{2}(t+T)$ também é solução, portanto

$$
y_{2}(t+T)=y_{2}(T) y_{1}(t)+y_{2}(t)
$$

usamos que $\dot{y}_{2}(n T)=1$.

Para $t=n T$, como $y_{1}(n T)=y_{1}(0)=1$ temos

$$
y_{2}((n+1) T)-y_{2}(n T)=y_{2}(T) .
$$


Portanto,

$$
y_{2}(n T)=n y_{2}(T), \quad \text { para cada } n \in \mathbb{Z} .
$$

Donde segue que

$$
\phi(n T)=\phi(0)+n \phi^{\prime}(0) y_{2}(T), \quad \text { para cada } \quad n \in \mathbb{Z} .
$$

Fazendo $t=-T / 2$ em $y_{2}(t+T)$, temos que $y_{2}(T)=0$ se e somente se $y_{2}(T / 2)=0$.

Assim, segue o resultado:

Proposição 3.2.1. Ou todas as soluções da equação (3.8) são periódicas de período $T$, ou cada solução $t \mapsto \phi(t)$, com $\phi^{\prime}(0) \neq 0$ é ilimitada. O primeiro caso ocorre se, e somente, se $y_{2}(T)=0$ ou, equivalentemente, $y_{2}(T / 2)=0$.

Esta proposição foi enunciada e demonstrada originalmente em [BNZ86]. Ela mostra que a estabilidade da origem do sistema (3.7) é equivalente à total periodicidade, ou seja, cada solução numa vizinhança da origem é periódica de período $T$.

Utilizando a integral das áreas, concluí-se que a origem do sistema é estável se e somente se cada solução numa vizinhança da origem é periódica de menor período $T$.

Além da Proposição 3.2.1, os autores encontram uma classe de funções em que a instabilidade ocorre. Em particular, o equilíbrio é instável se a função $g$ definida por

$$
g(0)=\frac{f(0)}{2}, g(x)=\frac{V(x)}{x^{2}}, \quad \text { para } x \neq 0 .
$$

tem um mínimo isolado extremo em $x=0$.

O resultado principal do artigo é obtido no caso em que $f$ é par.

Teorema 3.2.1. Seja $f$ par. A origem do sistema (3.7) é estável se e somente se $f$ é localmente constante em zero, ou seja, somente no caso trivial.

Em [BNdOC88] é obtido um caso de estabilidade não trivial. Para isso, usou-se conceito de força pseudo-conservativa.

Definição 3.2.1. Considere o sistema (1.2). Se existe $\Pi: \Omega \mapsto \mathbb{R}, \Pi \in C^{2}$, tal que

$$
-\Pi_{x}=\alpha X+\gamma Y, \quad-\Pi_{y}=\gamma X+\beta Y
$$

então a força $F(X, Y)$ é chamada de pseudo-conservativa. A função $\Pi$ é chamada de pseudopotencial.

A partir da generalização de alguns teoremas conhecidos de mecânica, os autores mostram o seguinte:

Proposição 3.2.2. Os sistemas do tipo (1.2), tais que a força $F$ é pseudo-conservativa possuem integrais primeiras do tipo:

$$
V(x, \dot{x}, y, \dot{y})=\alpha(x, y) \dot{x}^{2}+2 \gamma(x, y) \dot{x} y+\beta(x, y) \dot{y}^{2}+\Pi(x, y) .
$$


em que

$$
\alpha=a+b y+c y^{2}, \quad \beta=d+e x+c x^{2}, \quad \gamma=k-\frac{1}{2} b x-\frac{1}{2} e y-c x y
$$

com $a, b, c, d, e, k$ constantes.

Além disso, vale que

Proposição 3.2.3. Se a função $V$ é uma integral primeira de (1.2), então existem $a, b, c, d, e, k \in \mathbb{R}$, tais que

$$
(b+2 c y) X-(e+2 c x) Y+\left(k-\frac{1}{2} b x-\frac{1}{2} e y-c x y\right)\left(Y_{y}-X_{x}\right)+\left(a+b y+c y^{2}\right) X_{y}-\left(d+e x+c x^{2}\right) Y_{x}=0 \text {. }
$$

Para sistemas do tipo (3.7), a igualdade (3.9) é

$$
\left[\frac{3}{2} e f+\left(d+\frac{1}{2} e x\right) f^{\prime}\right] y+\left[-\frac{3}{2} b f+\left(k-\frac{1}{2} b x\right) f^{\prime}\right] x=0
$$

Em particular, para $y=0$,

$$
-\frac{3}{2} b f\left(k-\frac{1}{2} b x\right) f^{\prime}=0
$$

o que implica

$$
-\frac{3}{2} e f\left(d+\frac{1}{2} e x\right) f^{\prime}=0
$$

Das equações (3.10) e (3.11), temos, respectivamente

$$
f(x)=\frac{c_{1}^{3}}{(2 k-b x)^{3}}, \quad f(x)=\frac{c_{2}^{3}}{(2 d+e x)^{3}},
$$

o que implica em

$$
b p+2 q k=0, \quad e p-2 d q=0, \quad f(x)=\frac{1}{(p+q x)^{3}} .
$$

Neste caso, o sistema (3.7) possui três integrais primeiras distintas.

Por exemplo, para $(a=1, b=c=d=e=k=0)$,

$$
\frac{1}{2} \dot{x}^{2}+\frac{x^{2}}{(2 p)(p+q x)^{2}}
$$

Utilizando essas integrais primeiras é provado que:

Proposição 3.2.4. A origem para o sistema (3.7) com $f(x)$ como em (3.12) é estável para qualquer p positivo.

A proposição acima apresenta um caso estabilidade não trivial. Observe que a classe de sistemas dada por $f(x)$ da forma (3.12) é muito especial, pois somente nesses casos ocorre a conservação da pseudo-energia.

O resultado principal de [BNdOC88] apresenta uma condição necessária para estabilidade da origem.

Proposição 3.2.5. Se $f \in C^{1}$ e $f^{\prime \prime}(0)$ existe, a condição

$$
3 f(0) f^{\prime \prime}(0)=4 f^{\prime}(0)^{2}
$$


é necessária para estabilidade da origem de (3.7).

Isto mostra que para esses sistemas a instabilidade é genérica.

Surge a pergunta: Para que funções, além da função constante e das funções da forma (3.12), a estabilidade da origem de (3.7) ocorre?

Em [Zam88], G. Zampieri construiu a classe de todos os casos estáveis. Esta é bastante restrita, dado arbitrariamente $\bar{f} \in C^{0}\left(\mathbb{R}_{+}, \mathbb{R}\right)$ com $\bar{f}(0)>0$, existe uma extensão contínua, de $\bar{f}, f: \mathbb{R} \mapsto$ $\mathbb{R}$, para qual a estabilidade ocorre. Além disso, quaisquer duas dessas extensões coincidem numa vizinhança da origem.

Esta construção envolve a utilização de uma "função chave", $k$, definida em termos das funções $u$ e $h$ (veja Seção 3.1) e alguns resultados de [BNZ86]. Não iremos reproduzí-la aqui, uma vez que esta não será utilizada na obtenção dos nossos resultados.

Para finalizar, ressaltamos que para sistemas (3.7) a origem é estável se e somente se cada solução numa vizinhança da origem é periódica com menor período T. Em outras palavras, a origem do sistema é estavél se e somente se o sistema possui $\tau\left(x_{0}\right)$-isocronismo.

\subsubsection{Sistemas do tipo: $X(x, y)=-x f(x), Y(x, y)=-y g(x)$}

Os sistemas deste tipo foram estudados sob dois principais aspectos: estabilidade e coexistência de soluções periódicas.

Começaremos com a estabilidade. Iremos apresentar os principais resultados dos artigos [BNdOC91] e [BNdOC92]. O objetivo desses é encontrar condições sobre a funções $f$ e $g$ que impliquem na estabilidade da origem do sistema.

Neste sentido, tem-se também os artigos [BNdOCG02] e [Zam11]. Devido à importância desses para o desenvolvimento da tese, reservamos o próximo capítulo somente para exposição dos mesmos. Considere o sistema

$$
\begin{gathered}
\left\{\begin{array}{l}
\ddot{x}=-x f(x), \\
\ddot{y}=-y g(x),
\end{array}\right. \\
f, g: U \mapsto \mathbb{R}, 0 \in U=\stackrel{\circ}{U} \subset \mathbb{R}, f, g \in C^{2}, f(0)>0, g(0)>0 .
\end{gathered}
$$

Esse sistema escrito como equação de primeira ordem em $\mathbb{R}^{4}$ fica dado por:

$$
\left\{\begin{array}{l}
\dot{x}=z_{1} \\
\dot{y}=z_{2} \\
\dot{z}_{1}=-x f(x) \\
\dot{z}_{2}=-y g(x) .
\end{array}\right.
$$

Como é tradicional na literatura, veja os artigos citados e o livro [MS66], iremos tratá-lo como sistema de segunda ordem, ou seja, da forma (3.13).

Como $f(0)>0$, então existe $\bar{x}_{0}$ tal que $\forall x_{0}$, com $0<x_{0}<\bar{x}_{0}$, a solução $x\left(t ; x_{0}\right)$ de $(3.13)_{1}$, com $x\left(0 ; x_{0}\right)=x_{0}$ e $\dot{x}\left(0 ; x_{0}\right)=0$ é $\tau\left(x_{0}\right)$ - periódica e par.

Substituindo $x\left(t ; x_{0}\right)$ em $(3.13)_{2}$, obtemos uma família de equações de Hill,

$$
\ddot{y}=-y g\left(x\left(t ; x_{0}\right)\right),
$$


indexada por $x_{0}$.

Como $x\left(t, x_{0}\right)$ é par, estamos no caso simétrico das Equações de Hill.

Sejam $y_{1}\left(t ; x_{0}\right)$ e $y_{2}\left(t ; x_{0}\right)$ as soluções normalizadas de $(3.14)$ satisfazendo as condições $(2.3)$. Essas funções $y_{1}\left(t ; x_{0}\right)$ e $y_{2}\left(t ; x_{0}\right)$ são continuamente diferenciáveis e satisfazem a igualdade do Wronskiano

$$
y_{1}\left(t ; x_{0}\right) \dot{y}_{2}\left(t ; x_{0}\right)-y_{2}\left(t ; x_{0}\right) \dot{y}_{1}\left(t ; x_{0}\right)=1, \quad \forall t \in \mathbb{R} .
$$

No caso $f=g$ o sistema possui duas integrais primeiras, a integral da energia mecânica de (3.13) e a integral das áreas. No caso $f \neq g$ não ocorre a conservação do movimento angular, isto é, o sistema não possui a integral das áreas.

Em [BNdOC91] é introduzido o conceito de integral das áreas generalizadas.

Definição 3.2.2. Dizemos que o sistema (3.13) admite uma integral das áreas generalizada se existem uma vizinhança aberta da origem $W \subset U$ e uma função de classe $C^{2}, s$,

$$
s: W \backslash\{(0,0)\} \mapsto \mathbb{R},
$$

$s(x, \dot{x})$ não nula em cada vizinhança de $(0,0)$, e tal que

$$
V=s(x, \dot{x}) \dot{y}-y \dot{s}(x ; \dot{x})
$$

é uma integral primeira (3.13), onde $\dot{s}(x ; \dot{x})=s_{x}(x, \dot{x}) \dot{x}-x f(x) s_{\dot{x}}(x, \dot{x})$.

A seguir os autores apresentam condições necessárias e suficientes para existência de integral das áreas generalizadas para (3.13). Destacamos a seguinte:

Teorema 3.2.2. Supondo $f(0)>0, g(0)>0$ e $0<\delta<\bar{x}_{0}$. O sistema (3.13) possui integral das áreas generalizadas, $s$, se e somente se, para todo $\epsilon>0$, existe $\left(a_{0}, b_{0}\right) \subset(0, \delta), a_{0}<\epsilon$, e $i \in\{1 ; 2\}$ tal que $\forall x_{0} \in\left(a_{0}, b_{0}\right), y_{i}\left(t ; x_{0}\right)$ é periódica de período $\tau\left(x_{0}\right)$.

Utilizando essas condições são construídas funções de Liapunov para o sistema (3.13), nos casos de total periodicidade e quando $4 g(0) \neq n^{2} f(0)$, para todo natural $n$. Mais precisamente, são obtidos os resultados:

Teorema 3.2.3. Se $f(0)>0, g(0)>0$ e $y_{i}\left(t ; x_{0}\right), i \in\{1,2\}$ são periódicas de período $\tau\left(x_{0}\right)$ para todo $x_{0}, 0<x_{0}<\delta$, com $\delta<\bar{x}_{0}$, então o sistema (3.13) admite uma função de Liapunov para estabilidade da origem.

Teorema 3.2.4. Se $f(0)>0, g(0)>0$ e $4 g(0) \neq n^{2} f(0)$, para todo $n \in \mathbb{N}$, então o sistema (3.13) admite uma função de Liapunov para estabilidade da origem. Estes casos são aperiódicos, isto é, existem soluções não periódicas de perído $\tau\left(x_{0}\right)$ perto da origem. Em particular $y_{1}\left(t, x_{0}\right)$ e $y_{2}\left(t, x_{0}\right)$ não são periódicas.

O Teorema 3.2.4 mostra que a estabilidade da origem é genérica para (3.13).

Como vimos, (3.14) é uma família de equações de Hill indexada por $x_{0}$. Da Teoria de Floquet para o caso simétrico (veja [MS66]), temos que $\dot{y}_{2}\left(\tau\left(x_{0}\right) ; x_{0}\right)=y_{1}\left(\tau\left(x_{0}, x_{0}\right)\right)$ e também são válidos os resultados de estabilidade da origem $y=0, \dot{y}=0$ para $x_{0}$-seção:

a) se $y_{1}^{2}\left(\tau\left(x_{0}\right) ; x_{0}\right)>1$, então a origem é instável; 
b) se $y_{1}^{2}\left(\tau\left(x_{0}\right) ; x_{0}\right)<1$, então a origem é estável.

c) se $y_{1}^{2}\left(\tau\left(x_{0}\right) ; x_{0}\right)=1$ e $y_{2}\left(\tau\left(x_{0}\right) ; x_{0}\right)=\dot{y}_{1}\left(\tau\left(x_{0}\right), x_{0}\right)=0$, então a origem é estável.

d) se $y_{1}^{2}\left(\tau\left(x_{0}\right) ; x_{0}\right)=1$ e $y_{2}^{2}\left(\tau\left(x_{0}\right) ; x_{0}\right)+\dot{y}_{1}^{2}\left(\tau\left(x_{0}\right), x_{0}\right)>0$, então a orgem em instável.

Esses resultados seguem do Teorema 2.1.1.

Em [BNdOC92], utilizando a identidade do Wronskiano, os resultados de estabilidade acima são reescritos em termos da função à valores reais:

$$
l\left(x_{0}\right)= \begin{cases}-\frac{\dot{y}_{1}\left(\tau\left(x_{0}\right) ; x_{0}\right)}{y_{2}\left(\tau\left(x_{0}\right) ; x_{0}\right)} & \text { se } \quad y_{2}\left(\tau\left(x_{0}\right) ; x_{0}\right) \neq 0 \\ g\left(x_{0}\right) & \text { se } \quad y_{2}\left(\tau\left(x_{0}\right) ; x_{0}\right)=\dot{y}_{1}\left(\tau\left(x_{0}\right), x_{0}\right)=0 \\ +\infty & \text { se } \quad y_{2}\left(\tau\left(x_{0}\right) ; x_{0}\right) \neq \dot{y}_{1}\left(\tau\left(x_{0}\right), x_{0}\right)=0 .\end{cases}
$$

E são obtidos os critérios de estabilidade:

Teorema 3.2.5 (Critério de Estabilidade para $x_{0}$ Seção). Uma condição necessária e suficiente para estabilidade da $x_{0}$ seção é:

$$
0<l\left(x_{0}\right)<+\infty
$$

Teorema 3.2.6 (Critério Fundamental de Estabilidade). Uma condição necessária e suficiente para a estabilidade da solução $x=y=\dot{x}=\dot{y}=0$ de (4.1) é

$$
0<\liminf _{x_{0} \rightarrow 0^{+}} l\left(x_{0}\right) \leq \limsup _{x_{0} \rightarrow 0^{+}} l\left(x_{0}\right)<+\infty
$$

O Teorema 3.2.6 permite obter informações sobre a estabilidade da origem a partir da análise do comportamento da função $l$ numa vizinhança de zero.

Em [BNdOCG02], os Teoremas 3.2.5 e 3.2.6 são utilizados para obtenção de um novo Critério de Estabilidade, do qual são extraídas condições necessárias e/ou suficientes para estabilidade da origem em termos de $f(0), g(0), f^{\prime}(0), f^{\prime \prime}(0), g^{\prime}(0), g^{\prime \prime}(0)$.

No aspecto de coexistência de soluções periódicas destacam-se os trabalhos de G. Zampieri: [Zam89] e [Zam99]. Iremos apresentá-los sem muitos detalhes, o que a primeira vista parece estranho, uma vez que estamos interessados em um tipo especial de coexistência de soluções periódicas. O motivo para isso é que a abordagem utilizada nesses trabalhos é diferente da que utilizamos na tese.

Definição 3.2.3. Considere (3.14), com $x_{0}$ fixado. Dizemos que ocorre a coexistência de soluções periódicas se todas as soluções de (3.14) são periódicas de mesmo período $\tau\left(x_{0}\right)$.

Como consequência dos Teoremas 3.2.2 e 3.2.3, concluí-se que: Se o sistema admite integral das áreas generalizadas (veja Definição 3.2.2), então estabilidade é equivalente a coexistência de soluções periódicas para cada equação de Hill (3.14). Neste caso, a tripla $(f, g, s)$ é denotada de coexistence-like.

Sempre que $(f, g, s)$ é coexistence-like, dadas $f$ e $s$ existe uma relação entre $f, s$ e $g$ que determina $g$ unicamente.

Algumas funções $s$ são tais que para cada $f$ existe $g$, unicamente determinada por $f$ e $s$, tal que $(f, g, s)$ é coexistence-like. 
Cada uma dessas $s$ determina o seguinte problema, conhecido como free coexistence-like: Determinar todas as funções $f$ de classe $C^{1}$, definidas numa vizinhança de $0 \in \mathbb{R}$ e com $f(0)>0$, tal que a origem é um equilibrio estável para (3.13) (com g determinada unicamente por $f$ e $s$ ).

Existem quatro famílias de free coexistence-like:

1. $s(x, \dot{x})=x+\sigma, \quad g(x)=\frac{x f(x)}{x+\sigma}, \quad \sigma \in \mathbb{R} \backslash\{0\} ;$

2. $s(x, \dot{x})=x, \quad g(x)=f(x)$;

3. $s(x, \dot{x})=(1+\alpha x) \dot{x}, \quad g(x)=\left(1+\frac{3 \alpha x}{1+\alpha x}\right) f(x)+x f^{\prime}(x), \quad \alpha \in \mathbb{R} ;$

4. $s(x, \dot{x})=x \dot{x}, \quad g(x)=4 f(x)+x f^{\prime}(x)$.

G.Zampieri resolveu todos os casos de free coexistence-like, ou seja, para cada caso acima encontrou todas as funções $f(x)$ para as quais a origem é estável.

No caso 1) é fácil ver que ocorre instabilidade para toda função $f$. O problema para os caso 2),3) e 4) são não-triviais e foram resolvidos, respectivamente em [BNdOC88],[Zam89] e [Zam99].

Para mais informações sobre a coexistência de soluções periódicas, sugerimos a leitura do Capítulo 7 de [MS66]. 


\section{Capítulo 4}

\section{Condições computáveis para estabilidade da origem de sistemas posicionais e isocronismo}

Este capítulo é dedicado à apresentação dos artigos: [BNdOCG02] e [Zam11]. O primeiro forneceu ferramentas essenciais para o desenvolvimento desta tese. Os resultados que obtemos, no Capítulo 5, são dados em termos das derivadas de ordem $k$ em zero das funções $\tau, t_{1}$ e $t_{2}$, as duas últimas definidas nesse artigo. Além disso, é apresentado um método que permite calcular as derivadas até a segunda ordem dessas funções em zero. A partir da aplicação sucessiva do método, obtivemos informações importantes sobre as derivadas de ordem mais alta dessas funções, que foram fundamentais para a demonstração dos nossos principais resultados.

Em [Zam11], Zampieri apresenta uma classe de sistemas hamiltonianos autônomos de 2 graus de liberdade e descreve toda a dinâmica desses sistemas. Estamos interessados nas condições necessárias de estabilidade obtidas, as quais mostramos, no Capítulo 7, serem iguais às condições necessárias para ocorrência de $\tau\left(x_{0}\right)$ - isocronismo que obtemos ao aplicar nossos resultados.

\subsection{Um método computacional para a estabilidade na origem}

Vamos, nesta seção, apresentar os resultados do artigo "A Computational Method for the Stability of a Class of Mechanical Systems" dos autores Angelo Barone- Netto, Mauro de Oliveira César e Gianluca Gorni [BNdOCG02], que serão úteis em nosso trabalho.

O objetivo deste artigo é estudar a estabilidade da origem para sistemas do tipo

$$
\left\{\begin{array}{l}
\ddot{x}=-x f(x), \\
\ddot{y}=-y g(x),
\end{array}\right.
$$

com $f$ e $g$ de classe $C^{2}$, definidas numa vizinhança aberta de $0 \in \mathbb{R}$, tais que $f(0)>0, g(0)>0$ e $4 g(0)=n^{2} f(0)$, para algum $n \in \mathbb{N}$.

Lembramos que o caso $4 g(0) \neq n^{2} f(0)$, para todo $n \in \mathbb{N}$, foi estudado em [BNdOC88], no qual mostrou-se que estes casos são estáveis.

O resultado principal é chamado pelos autores de Novo Critério de Estabilidade (variante do 
Teorema 3.1 de [BNdOC91]), são dadas condições suficientes de estabilidade ou instabilidade para a origem, a partir da análise do valor assumido por uma função que caracteriza a estabilidade, $\tilde{l}$. Esta é definida em termos da função período $\tau$ e das funções $t_{1}$ e $t_{2}$, apresentadas pelos autores no texto, chamadas de tempos de parada.

Com a aplicação do Novo Critério de Estabilidade são obtidas condições necessárias ou suficientes para estabilidade da origem em termos de um sistema de desigualdades em $f(0), g(0) f^{\prime}(0), g^{\prime}(0)$, $f^{\prime \prime}(0)$ e $g^{\prime \prime}(0)$.

Como veremos, para a obtenção dessas condições de estabilidade é preciso calcular as derivadas $\tau, t_{1}$ e $t_{2}$ em 0 até a segunda ordem. Os autores apresentam um método para estes cálculos, utilizando equações variacionais. Deixamos a apresentação desse método para o próximo capítulo, em que fazemos explicitamente os cálculos para as derivadas dessas funções na origem até a ordem 4 .

Além das condições para estabilidade, são apresentadas várias aplicações interessantes do Novo Critério de Estabilidade. Dentre elas é obtida novamente a condição suficiente de instabilidade para o caso $f=g$, apresentada por Barone e Mauro em [BNdOC88].

Daremos ênfase ao resultado principal do artigo e as definições que utilizaremos para construção dos nossos resultados. Destacamos em especial às definições das funções $\tau, t_{1}$ e $t_{2}$, bem como a suas propriedades.

\subsubsection{As funções tempo de parada}

O resultado principal de [BNdOCG02] é baseado em duas funções, $t_{1}$ e $t_{2}$. Nesta subseção apresentaremos as definições e algumas propriedades dessas funções.

Sem perda de generalidade, toma-se $f(0)=1$ e são estudados os casos em que $4 g(0)=n^{2}$, para algum $n \in \mathbb{N}$.

As funções $t_{1}$ e $t_{2}$ são definidas como:

$$
\begin{aligned}
& t_{1}\left(x_{0}\right)=n \text {-ésimo zero estritamente positivo de } \dot{y}_{1}\left(., x_{0}\right) . \\
& t_{2}\left(x_{0}\right)=n \text {-ésimo zero estritamente positivo de } y_{2}\left(., x_{0}\right) .
\end{aligned}
$$

A Proposição provada a seguir corresponde à Proposição 3.1 de [BNdOCG02].

Proposição 4.1.1. Para cada $x_{0}$ suficientemente pequeno, $t_{1}\left(x_{0}\right)$ e $t_{2}\left(x_{0}\right)$ estão bem definidas e são suaves com respeito a $x_{0}$, e $t_{1}(0)=t_{2}(0)=2 \pi$.

Demonstração. As soluções fundamentais $y_{1}\left(t ; x_{0}\right)$ e $y_{2}\left(t ; x_{0}\right)$ de $(3.14)$ são suaves com respeito a $\left(t ; x_{0}\right)$. Em particular, para $x_{0}=0$

$$
y_{1}(t, 0)=\cos \left(\frac{n t}{2}\right) \quad \text { e } \quad y_{2}(t, 0)=\frac{2}{n} \sin \left(\frac{n t}{2}\right) .
$$

Portanto, $t_{1}(0)=t_{2}(0)=2 \pi$. Como $\dot{y}_{1}(t, 0)=0$ então $\ddot{y}_{1}(t, 0) \neq 0$, seus zeros são isolados e, além disso, pode-se aplicar o Teorema da Função Implícita para equação $\dot{y}_{1}\left(t ; x_{0}\right)=0$ na incógnita. Concluí-se assim que, para $x_{0}$ suficientemente pequeno, a função $t \mapsto \dot{y}_{1}\left(t ; x_{0}\right)$ também tem o $n$ ésimo zero no tempo $t_{1}\left(x_{0}\right)$ perto de $2 \pi$, dependendo suavemente (pelo menos $C^{1}$ ) de $x_{0}$. A prova para $t_{2}\left(x_{0}\right)$ é análoga. 


\subsubsection{Resultados de estabilidade}

Usando as funções $t_{1}$ e $t_{2}$ os autores definem, para $x_{0}$ suficientemente pequeno, uma nova função que caracteriza a estabilidade:

$$
\tilde{l}\left(x_{0}\right)= \begin{cases}-\frac{\tau\left(x_{0}\right)-t_{1}\left(x_{0}\right)}{\tau\left(x_{0}\right)-t_{2}\left(x_{0}\right)} & \text { se } t_{2}\left(x_{0}\right) \neq \tau\left(x_{0}\right) \\ 1 & \text { se } t_{1}\left(x_{0}\right)=t_{2}\left(x_{0}\right)=\tau\left(x_{0}\right) \\ +\infty & \text { se } t_{1}\left(x_{0}\right) \neq t_{2}\left(x_{0}\right)=\tau\left(x_{0}\right)\end{cases}
$$

Utilizando está função é obtido o principal resultado do artigo:

Teorema 4.1.1. (Novo Critério de Estabilidade) A origem $(y, \dot{y})=(0,0)$ é um equilíbrio estável para a família de equações de Hill (3.14) se e somente se:

$$
0<\tilde{l}\left(x_{0}\right)<+\infty
$$

A origem $(x, \dot{x}, y, \dot{y})=(0,0,0,0)$ é um equilibrio estável de (4.1) se e somente se

$$
0<\liminf _{x_{0} \rightarrow 0^{+}} \tilde{l}\left(x_{0}\right) \leq \limsup _{x_{0} \rightarrow 0^{+}} \tilde{l}\left(x_{0}\right)<+\infty
$$

O quociente que define $\tilde{l}$ tem, no caso principal, a forma indeterminada $0 / 0$ quando $x_{0} \mapsto 0$. Para aplicar a Regra de l'Hôpital é preciso calcular as derivadas de $\tau, t_{1}$ e $t_{2}$ em $x_{0}=0$. Como dito anteriormente, deixaremos esses cálculos para o próximo capítulo.

Os resultados do Teorema 4.1.1 são análogos aos já enunciados no Capítulo 3, Teoremas 3.2.5 e 3.2.6, trocando-se $l$ por $\tilde{l}$. Contudo, o Teorema 4.1.1 nos fornece condições de estabilidade menos abstratas que dos outros teoremas, uma vez que conhecidas as derivadas de $\tau, t_{1}$ e $t_{2}$ em 0 , as condições ficam dadas em função das derivadas de $f$ e $g$ em 0 , como obtém-se a seguir:

Teorema 4.1.2. Suponha que $f$ e $g$ são de classe $C^{2}$ e $g(0)=n^{2} f(0) / 4>0$ para algum $n \in \mathbb{N}$. Sejam

$$
\begin{aligned}
p_{1} & :=20 f^{\prime}(0)^{2}-9 f^{\prime \prime}(0)+24 g^{\prime \prime}(0), \\
p_{2} & :=20 f^{\prime}(0)^{2}-10 f^{\prime}(0) g^{\prime}(0)-10 g^{\prime}(0)^{2}+9\left(g^{\prime \prime}(0)-f^{\prime \prime}(0)\right), \\
p_{3} & :=20 f^{\prime}(0)^{2}-14 f^{\prime}(0) g^{\prime}(0)+2 g^{\prime}(0)^{2}+3\left(g^{\prime \prime}(0)-3 f^{\prime \prime}(0)\right), \\
p_{4} & :=n^{4}\left(20 f^{\prime}(0)^{2}-9 f^{\prime \prime}(0)\right)+24\left(2 f^{\prime}(0) g^{\prime}(0)-2 g^{\prime}(0)^{2}\right)-g^{\prime \prime}(0) \\
& +n^{2}\left(9 f^{\prime \prime}(0)+24 g^{\prime \prime}(0)-20 f^{\prime}(0)^{2}-48 f^{\prime}(0) g^{\prime}(0)\right) .
\end{aligned}
$$

\section{Então}

a) Caso $n=1$ : Se $g^{\prime}(0) \neq 0$, então a origem é um equilíbrio estável; se $g^{\prime}(0)=0$ e $p_{1} \neq 0$, então a origem é estável.

b) Caso $n=2$ : Se $p_{2} p_{3}>0$, então a origem é estável; se ou $p_{2} p_{3}<0$ ou somente um dos dois é nulo, então a origem é instável.

c) Caso $n \geq 3:$ Se $p_{4} \neq 0$, então a origem é estável. 
Apresentaremos a demonstração do caso $n=2$, o qual futuramente será do nosso interesse. Para as fórmulas das derivadas de $\tau, t_{1}$ e $t_{2}$ veja o Teorema 5.2.1 desta tese.

Demonstração. Para $n=2$, temos $\tau^{\prime}(0)-t_{1}^{\prime}(0)=\tau^{\prime}(0)-t_{2}^{\prime}(0)=0 \mathrm{e}$

$$
\tau^{\prime \prime}(0)-t_{1}^{\prime \prime}(0)=\frac{\pi p_{2}}{12}, \quad \tau^{\prime \prime}(0)-t_{2}^{\prime \prime}(0)=\frac{\pi p_{3}}{12} .
$$

Então, se $p_{3} \neq 0, t_{2}\left(x_{0}\right) \neq \tau\left(x_{0}\right)$ para $x_{0} \neq 0$ suficientemente pequeno e

$$
\lim _{x_{0} \rightarrow 0^{+}} \bar{l}\left(x_{0}\right)=\frac{\tau^{\prime \prime}(0)-t_{1}^{\prime \prime}(0)}{\tau^{\prime \prime}(0)-t_{2}^{\prime \prime}(0)}=\frac{\pi p_{2} / 12}{\pi p_{3} / 12} .
$$

Utilizando o Novo Critério de Estabilidade, tem-se que: se $p_{2} p_{3}>0$, então a origem é estável, e se $p_{2} p_{3} \leq 0$ a origem é instável.

Se $p_{2} \neq 0$ e $p_{3}=0$ observe que:

- Se $t_{2}\left(x_{0}\right) \neq \tau\left(x_{0}\right)$ em um intervalo $(0, \delta)$, para algum $\delta>0$, então é claro $\lim _{x_{0} \rightarrow \infty}\left|\bar{l}\left(x_{0}\right)\right|$ é $+\infty$, o que garante a instabilidade.

- Se $t_{2}$ e $\tau$ são iguais em uma sequência $x_{0}^{n} \mapsto 0^{+}$, como $p_{2} \neq 0$, tem-se $\tau\left(x_{0}^{n}\right) \neq t_{1}\left(x_{0}^{n}\right)$, portanto $\bar{l}\left(x_{0}^{n}\right)=+\infty$ e outra vez há instabilidade.

\subsection{Isocronismo para uma classe de sistemas hamiltonianos}

Nesta seção apresentaremos alguns resultados obtidos por Gaetano Zampieri no artigo: "Completely Integrable Hamiltonian Systems with Weak Lyapunov Instability or Isocrony" [Zam11].

Nesse notável trabalho o autor introduz a classe de sistemas hamiltonianos autônomos de dois graus de liberdade:

$$
\left\{\begin{array}{l}
\dot{q}_{1}=p_{2} \\
\dot{q}_{2}=p_{1} \\
\dot{p}_{1}=-F^{\prime}\left(q_{1}\right) q_{2} \\
\dot{p}_{2}=-F\left(q_{1}\right) .
\end{array}\right.
$$

A função Hamiltoniana do sistema é:

$$
H(q, p)=p_{1} p_{2}+F\left(q_{1}\right) q_{2}
$$

Zampieri mostra que estes sistemas são completamente integráveis e descreve sua dinâmica em todos os detalhes.

Para estes sistemas a instabilidade é genérica. Tem-se um tipo especial de instabilidade, todas as órbitas do sistema linearizado de (4.2) na origem em $\mathbb{R}^{4}$ são limitadas e não existem movimentos assintóticos para o equilíbrio, ou seja, nenhuma solução não-constante tem a origem como ponto limite quando $t \mapsto-\infty$. Este tipo de instabilidade foi denominada por Zampieri de Instabilidade Fraca. 
Por outro lado, a estabilidade é rara e caracterizada pela presença de um centro isócrono no plano $q_{1}, p_{2}$. Isto é, uma condição necessária e suficiente para estabilidade da origem de (4.2) é que todas as órbitas numa vizinhança de $(0,0)$ no plano $q_{1}, p_{2}$ tenham o mesmo período. Neste caso, todas as órbitas de $(4.2)$ em $\mathbb{R}^{4}$, com $\left(q_{1}, p_{2}\right)$ perto de $(0,0)$, são periódicas e têm o mesmo período. Lembramos que um centro isócrono é aquele cuja função período é constante. Assim, dizemos que um sistema é isócrono se sua função período for constante.

Restringimo-nos a apresentar Seção 2 de [Zam11] e alguns resultados de estabilidade. Na Subseção 4.2.2 são obtidos resultados para centros isócronos bidimensionais. Dentre estes resultados são dadas condições necessárias para que o centro no plano $q_{1}, p_{2}$ seja isócrono, e consequentemente, a origem do sistema (4.2) seja estável.

\subsubsection{Isocronismo no plano}

Considere a equação

$$
\ddot{x}=-F(x), \quad F(0)=0, \quad F^{\prime}(0)>0,
$$

com $F$ contínua numa vizinhança de 0 em $\mathbb{R}$ e diferenciável em 0 .

O sistema (4.3) é conservativo, logo possui a integral de Energia:

$$
G(x, \dot{x})=\frac{\dot{x}^{2}}{2}+V(x), \quad V(x)=\int_{0}^{x} F(s) d s .
$$

Como vimos na Seção 3.1, a função período é

$$
\tau\left(x_{0}\right)=2 \int_{h\left(x_{0}\right)}^{x_{0}} \frac{d x}{\sqrt{2\left(V\left(x_{0}\right)\right)-V(x)}},
$$

em que $h$ é a involução associada com $V$ (veja a Seção 3.1).

Utilizando a função $\tau\left(x_{0}\right)$, são provados os resultados:

Teorema 4.2.1. (Determinando centros isócronos). Seja $V$ de classe $C^{1}$ perto de $0 \in \mathbb{R} ;$ assuma que exista $V^{\prime \prime}(0)>0$. Seja $J$ um intervalo aberto (como na Seção 3.1) e $h \in \operatorname{Diff}^{1}(J ; J)$ a involução associada com $V$. Então todas as órbitas de $\ddot{x}=-V^{\prime}(x)$ que intersectam o intervalo $J$ do eixo $x$, no plano $x, \dot{x}$, são periódicas e englobam a origem. Além disso, possuem um mesmo período se, e somente se,

$$
V(x)=\frac{V^{\prime \prime}(0)}{8}(x-h(x))^{2}, \quad x \in J .
$$

Neste caso, dizemos que a origem é um centro isócrono para $\ddot{x}=-V^{\prime}(x)$.

A partir de (4.5) e de propriedades da involução $h$ são obtidas algumas condições necessárias para a função período ser constante. Estas podem ser usadas para verificar quando uma função $F$ gera localmente um centro isócrono.

Corolário 4.2.1 (Condições necessárias). Seja $V$ tal que $V^{(6)}(0)$ exista, satisfazendo $V(0)=$ $V^{\prime}(0)=0, V^{\prime \prime}(0)>0$. Se, além disso, a origem é uma centro isócrono para $\ddot{x}=-V^{\prime}(x)$, então

$$
V^{(4)}(0)=\frac{5 V^{(3)}(0)}{3 V^{\prime \prime}(0)} \quad V^{(6)}(0)=\frac{7 V^{(3)}(0) V^{(5)}(0)}{V^{\prime \prime}(0)}-\frac{140 V^{(3)}(0)^{4}}{9 V^{(3)}(0)^{3}} .
$$


No nosso caso $F(x)=-x f(x)$, com $f$ definida e positiva numa vizinhança de 0 , de classe $C^{\infty}, f(0)=1 \mathrm{e}$

$$
G(x, \dot{x})=\frac{\dot{x}^{2}}{2}+V(x), \quad V(x)=\int_{0}^{x} s f(s) d s .
$$

Note que $x f(x)$ satisfaz todas as hipóteses do Corolário 4.2.1, assim seguem as condições necessárias para existência de um centro isócrono para $\ddot{x}=-x f(x)$ :

$$
f^{\prime \prime}(0)=\frac{20 f^{\prime}(0)^{2}}{9}, \quad f^{(4)}(0)=\frac{56 f^{(3)}(0) f^{\prime}(0)}{5}-\frac{448 f^{\prime}(0)^{4}}{9} .
$$

Uma outra maneira de se obter as condições (4.6) é derivar a função $\tau$ com respeito a $x_{0}$, calcular em $x_{0}=0$ e igualar a zero. Para isso, podemos usar as derivadas da função $\tau$ em 0 até a quarta ordem, Teorema 5.2.1.

\subsubsection{Resultados de Estabilidade}

Em [Zam11] são demonstrados alguns resultados de estabilidade para o sistema (4.2). Apresentaremos somente o enunciado destes resultados, provados por Zampiere naquele trabalho.

Considere o sistema de dois graus de liberdade definido pela função Langragiana

$$
\begin{gathered}
L(x, y, \dot{x}, \dot{y})=\dot{x} \dot{y}-F(x) y, \\
\ddot{x}=-F(x), \quad \ddot{y}=-y F^{\prime}(x) y, \quad F(0)=0, \quad F^{\prime}(0)>0,
\end{gathered}
$$

com $F \in C^{1}$ perto de 0 em $\mathbb{R}$. Este sistema possui duas integrais primeiras $G(x, \dot{x})$ e $F(x, y, \dot{x}, \dot{y})$

$$
\begin{gathered}
G(x, y, \dot{x}, \dot{y})=\frac{\dot{x}^{2}}{2}+V(x), \quad V(x)=\int_{0}^{x} F(s) d s, \\
F(x, y, \dot{x}, \dot{y})=\dot{y} \dot{x}+g(x) y .
\end{gathered}
$$

Fixando $x_{0} \in J, x_{0}>0$, seja $t \mapsto X\left(t ; x_{0}\right)$ a solução periódica de $(4.7)_{1}$ com condições iniciais $\left(x_{0}, 0\right)$. Substituindo $X\left(t ; x_{0}\right)$ em $(4.7)_{2}$, obtemos a equação de Hill,

$$
\ddot{y}=-F^{\prime}\left(X\left(t, x_{0}\right)\right) y
$$

Proposição 4.2.1. Seja $x_{0} \in J$, com $x_{0}>0$, então as soluções de (4.8) são todas $\tau\left(x_{0}\right)$ periódicas ou são todas ilimitadas, com exceção das que são proporcionais a $t \mapsto \partial_{t} X\left(t, x_{0}\right)$. Uma condição necessária e suficiente para que todas sejam periódicas de mesmo período $\tau\left(x_{0}\right)$ é que $\tau^{\prime}\left(x_{0}\right)=0$.

Zampieri concluiu que a origem em $\mathbb{R}^{4}$ é um equilíbrio instável de (4.7) se, e somente se, é possível encontrar $x_{0}>0$ arbitrariamente perto de 0 tal que a equação (4.8) tem soluções ilimitadas. Da Proposição 4.2.1, é o obtido o principal resultado de estabilidade para (4.7).

Teorema 4.2.2 (Estabilidade e Instabilidade Fraca). Seja $F$ uma função $C^{1}$ perto de 0 em $\mathbb{R}$ com $F(0)=0, F^{\prime}(0)>0$, então a origem em $\mathbb{R}^{4}$ é um equilíbrio estável para o sistema (4.7), se e somente se a origem em $\mathbb{R}^{2}$ é localmente um centro isócrono para $\ddot{x}=-F(x)$, neste caso todas as órbitas de $(4.7)$ com $(x, \dot{x})$ perto de $(0,0)$ são periódicas e possuem o mesmo período. Se o equilíbrio é instável, então existe uma sequência de condições inicias que convergem para a origem em $\mathbb{R}^{4}$, 
cujas soluções correspondentes são ilimitadas. A instabilidade é fraca, isto significa que não existe nenhum movimento assintótico para o equílibrio, ou seja, nenhuma solução não-constante tem a origem como ponto limite quando $t \rightarrow-\infty$. 
26 CONDIÇÕES COMPUTÁVEIS PARA ESTABILIDADE DA ORIGEM DE SISTEMAS POSICIONAIS E ISOCRONISMO 


\section{Capítulo 5}

\section{Condições necessárias e suficientes para o $\tau\left(x_{0}\right)$-isocronismo}

Estudaremos neste capítulo sistemas do tipo:

$$
\left\{\begin{array}{l}
\ddot{x}=-x f(x), \\
\ddot{y}=-y g(x),
\end{array}\right.
$$

$\operatorname{com}(f, g) \in C^{k}(J) \times C^{k}(J), J \subset \mathbb{R}$ intervalo aberto satisfazendo (3.4), $f(x)>0, g(x)>0$.

Nosso objetivo é encontrar condições para o $\tau\left(x_{0}\right)$-isocronismo, ou seja, fixada $f(x)$ procurar condições sobre $g(x)$ para existir $\delta>0$, tal que as soluções de (5.1) com condições iniciais: $x(0)=$ $x_{0}, \dot{x}(0)=0, y(0)=y_{0}, \dot{y}(0)=\dot{y}_{0}$ sejam $\tau\left(x_{0}\right)$ - periódicas, $\forall\left(x_{0}, y_{0}, \dot{y}_{0}\right) \neq(0,0,0)$, com $0 \leq x_{0}<\delta$.

Lembrando que $\tau\left(x_{0}\right)$-periódica significa que a solução é periódica de menor período $\tau\left(x_{0}\right)$.

Como estamos interessados em sistemas que possuam soluções periódicas numa vizinhança da origem, vamos nos concentrar em sistemas com $4 g(0)=n^{2} f(0)$, para algum $n \in \mathbb{N}$.

Na Seção 5.1 obtemos o resultado principal deste capítulo, o Teorema 5.1.2. Supondo $f$ e $g$ analíticas o teorema apresenta condições necessárias e suficientes, em termos das derivadas das funções $\tau, t_{1}$ e $t_{2}$ em zero, que garantem a ocorrência de $\tau\left(x_{0}\right)$-isocronismo para o sistema (5.1). Se $f$ e $g$ são funções de classe $C^{m}$ essas condições são apenas necessárias.

A seguir, na Seção 5.2 exibimos um método que permite obter as fórmulas para as derivadas das funções $\tau, t_{1}$ e $t_{2}$ em zero. Aplicando esse método sucessivamente, deduzimos expressões para $\tau^{(k)}(0), t_{1}^{(k)}(0)$ e $t_{2}^{(k)}(0), k$ natural.

Para finalizar, na Seção 5.3 apresentamos algumas propriedades dessas expressões, que serão importantes para demonstração do nosso resultado principal.

\subsection{Resultados preliminares}

\subsubsection{Introdução}

Nesta seção apresentamos o resultado principal deste capítulo.

Considere o sistema (5.1) satisfazendo as condições iniciais: $x(0)=x_{0} \geq 0, \dot{x}(0)=0, y(0)=y_{0}$ e $\dot{y}(0)=\dot{y}_{0}$. .

Para cada $x_{0} \in J$, seja $x\left(t ; x_{0}\right)$ a solução de $(5.1)_{1}$, com condições iniciais $x(0)=x_{0}, \dot{x}(0)=0$. 
Sabemos que $x\left(t ; x_{0}\right)$ está definida para todo $t \in \mathbb{R}$ e é periódica. Se $x_{0} \neq 0$, então $\tau\left(x_{0}\right)$ é o menor período de $x\left(t, x_{0}\right)$. Se $x_{0}=0$, então $\tau(0)=\lim _{x_{0} \rightarrow 0^{+}}=2 \pi / \sqrt{f(0)}$.

Substituindo $x\left(t, x_{0}\right)$ na segunda equação de $(5.1)$, obtemos uma família de equações de Hill

$$
\ddot{y}=-y g\left(x\left(t, x_{0}\right)\right),
$$

indexada por $x_{0}$.

Sejam $y_{1}\left(t, x_{0}\right)$ e $y_{2}\left(t, x_{0}\right)$ as soluções normalizadas de $(5.2)$, respectivamente, com

$$
\begin{array}{ll}
y_{1}\left(0 ; x_{0}\right)=1, & \dot{y}_{1}\left(0 ; x_{0}\right)=0 \\
y_{2}\left(0 ; x_{0}\right)=0 & \dot{y}_{2}\left(t ; x_{0}\right)=1 .
\end{array}
$$

Para cada $x_{0} \geq 0,(5.2)$ é uma equação de Hill, simétrica, pois $x\left(t, x_{0}\right)$ é par.

Na proposição a seguir, obtemos uma primeira condição necessária sobre a função $g(x)$ para que (5.1) apresente $\tau\left(x_{0}\right)$-isocronismo.

Proposição 5.1.1. Considere o sistema (5.1). Se ocorre $\tau\left(x_{0}\right)$-isocronismo, então $g(0)=f(0)$.

Demonstração. Para cada $x_{0} \in J$, a solução $x\left(t ; x_{0}\right)$ é $\tau\left(x_{0}\right)$ - periódica. A função período $\tau$ é suave e satisfaz: $\lim _{x_{0} \rightarrow 0^{+}} \tau\left(x_{0}\right)=2 \pi / \sqrt{f(0)}$. Considere a equação de Hill correspondente com condições inicias $y(0)=y_{0}, \dot{y}(0)=\dot{y}_{0}$.

Por hipótese, a solução $y\left(t ; x_{0}, 0, y_{0}, \dot{y}_{0}\right)$ é $\tau\left(x_{0}\right)$-periódica, ou seja,

$$
y\left(t+\tau\left(x_{0}\right), x_{0}, 0, y_{0}, \dot{y}_{0}\right)=y\left(t, x_{0}, 0, y_{0}, \dot{y}_{0}\right), \quad \forall t \in \mathbb{R} .
$$

Como (5.3) é válida para todo $x_{0} \in J$, vale quando fazemos $x_{0} \mapsto 0^{+}$, logo

$$
y\left(t+\lim _{x_{0} \rightarrow 0^{+}} \tau\left(x_{0}\right), 0,0, y_{0}, \dot{y}_{0}\right)=y\left(t, 0,0, y_{0}, \dot{y}_{0}\right)
$$

Usando que $\lim _{x_{0} \rightarrow 0^{+}} \tau\left(x_{0}\right)=2 \pi / \sqrt{f(0)}$, temos que o menor período de $y\left(t, 0,0, y_{0}, \dot{y}_{0}\right)$, se $\left(y_{0}, \dot{y}_{0}\right) \neq$ $(0,0)$, deve ser $2 \pi / \sqrt{f(0)}$.

Para $x_{0}=0$, temos que (5.2) é

$$
\ddot{y}=-y(t, 0) g(0)
$$

e as soluções fundamentais são

$$
y_{1}(t, 0)=\cos (\sqrt{g(0)} t) \quad \text { e } \quad y_{2}(t, 0)=\frac{1}{\sqrt{g(0)}} \sin (\sqrt{g(0)} t)
$$

cujo menor período é $\frac{2 \pi}{\sqrt{g(0)}}$. Portanto, $g(0)=f(0)$.

Sem perda de generalidade, consideraremos $f(0)=1$. Assim, uma condição necessária para (5.1) possuir $\tau\left(x_{0}\right)$-isocronismo é $g(0)=1$.

Da Teoria de Floquet, caso simétrico, veja o Capítulo 2, Teoremas 2.1.2 e 2.1.3, segue o resultado.

Teorema 5.1.1. Seja $w: \mathbb{R} \mapsto \mathbb{R}$ de classe $C^{k}, k \geq 2$, par e periódica de menor período $p>0$. Se $y_{1}(t)$ e $y_{2}(t)$ são as soluções normalizadas da equação de Hill $\ddot{y}=-w(t) y$, então $\dot{y}_{2}(p)=y_{1}(p)$ e, além disso: 
a) pelo menos uma das soluções normalizadas é periódica de menor período $p$ ou $2 p$;

b) $y_{1}(t)$ será periódica de menor período $p$ se, e só se, $\dot{y}_{1}\left(\frac{p}{2}\right)=0$;

c) $y_{1}(t)$ será periódica de menor período $2 p$ se, e só se, $y_{1}\left(\frac{p}{2}\right)=0$;

d) $y_{2}(t)$ será periódica de menor período $p$ se, e só se, $y_{2}\left(\frac{p}{2}\right)=0$;

e) $y_{2}(t)$ será periódica de menor período $2 p$ se, e só se, $\dot{y}_{2}\left(\frac{p}{2}\right)=0$;

f) todas as soluções periódicas pares dessa equação de Hill são múltiplas de $y_{1}$ e todas as soluções periódicas impares dessa equação são múltiplas de $y_{2}$;

g) as soluções de $\ddot{y}=-w(t) y$ serão todas periódicas, se, e só se, $y_{1}$ e $y_{2}$ forem periódicas. Nesta situação todas as soluções não triviais dessa equação terão o mesmo menor período.

A seguir expomos um resultado importante para a sequência do trabalho, pois esclarece um ponto que nos preocupava. Como $x\left(t ; x_{0}\right)$ é periódica de menor período $\tau\left(x_{0}\right)$, é claro que a função $g\left(x\left(t, x_{0}\right)\right)$ tem $\tau\left(x_{0}\right)$ como período, mas, a princípio, a única coisa que se pode afirmar sobre o menor período dessa função é que, se $g(x)$ é não constante, o menor período é $\frac{\tau\left(x_{0}\right)}{n}$ para algum natural $n$.

Entretanto, como $g(0)=1$, vai-se poder concluir em razão do Teorema da dependência contínua e diferenciável das soluções de e.d.o em relação a parâmetros e condições iniciais, e ao supracitado Teorema 5.1.1, que $n$ será 1 ou 2, como segue na Proposição 5.1.2.

Enfatizamos aqui que nessa proposição não se faz menção a $\tau\left(x_{0}\right)$-isocronismo, ela decorre apenas da hipótese $g(0)=1$.

Proposição 5.1.2. Se $g(0)=1$ e $g(x)$ não é constante em nenhuma vizinhança da origem, então existe $\delta>0$ tal que, para todo $0 \leq x_{0}<\delta$ o menor período de $g\left(x\left(t, x_{0}\right)\right)$ é $\tau\left(x_{0}\right)$ ou $\frac{\tau\left(x_{0}\right)}{2}$.

Demonstração. Suponha, como antes, $f(0)=1$. Assim, $g(0)=1$ e $\tau(0)=2 \pi$.

Como visto na prova da Proposição 5.1.1, $y_{1}(t, 0)=\cos (t)$ e $y_{2}(t, 0)=\sin (t)$. Pela definição de $y_{1}\left(t, x_{0}\right)$ e $y_{2}\left(t, x_{0}\right)$ tem-se $\dot{y}_{1}\left(0, x_{0}\right)=y_{2}\left(0, x_{0}\right)=0$. Segue-se do teorema da dependência contínua e diferenciável das soluções de equações diferenciáveis em relação a parâmetros e da continuidade de $\tau\left(x_{0}\right)$, que existe $\delta>0$ tal que, para $x_{0} \in[0, \delta)$, tem-se:

i) $\tau\left(x_{0}\right) \in\left(2 \pi-\frac{1}{2}, 2 \pi+\frac{1}{2}\right)$.

ii) $\dot{y}_{1}\left(t, x_{0}\right)$ e $y_{2}\left(t, x_{0}\right)$ anulam-se exatamente duas vezes em $\left(0,2 \pi+\frac{1}{2}\right)$, uma vez em $(\pi-1, \pi+1)$ e outra em $\left(2 \pi-\frac{1}{2}, 2 \pi+\frac{1}{2}\right)$.

iii) Como $\frac{4 \pi+1}{12}<\frac{\pi}{2}$, então pode-se escolher $\delta$ para que se $0 \leq x_{0}<\delta$, então $y_{1}\left(t, x_{0}\right) \dot{y}_{2}\left(t, x_{0}\right) \neq 0$ no intervalo $\left[0, \frac{4 \pi+1}{12}\right]$.

Agora veja que $\tau\left(x_{0}\right)$ é um período de $g\left(x\left(t, x_{0}\right)\right)$, assim, se $g$ não é constante então o menor período dessa função é $p_{x_{0}}=\frac{\tau\left(x_{0}\right)}{n\left(x_{0}\right)}$, com $n\left(x_{0}\right) \geq 1$ natural.

Por fim, vai-se provar que, se $0 \leq x_{0}<\delta$, então $n\left(x_{0}\right) \leq 2$. Suponha, por absurdo, que existe $x_{0} \in[0, \delta)$ para o qual $n\left(x_{0}\right) \geq 3$. Então $p_{x_{0}}=\frac{\tau\left(x_{0}\right)}{n\left(x_{0}\right)}<\frac{2 \pi+\frac{1}{2}}{3}=\frac{4 \pi+1}{6}$.

Como $g\left(x\left(t, x_{0}\right)\right)$ é par, o Teorema 5.1 .1 garante que pelo menos uma das soluções normalizadas de $\ddot{y}=-g\left(x\left(t, x_{0}\right) y\right), y_{1}$ ou $y_{2}$, é períódica com menor período $p_{x_{0}}$ ou $2 p_{x_{0}}$ e, dos itens $(b),(c),(d),(e)$ 
daquele resultado vem que pelo menos uma das funções $y_{1}\left(t, x_{0}\right), \dot{y}_{1}\left(t, x_{0}\right), y_{2}\left(t, x_{0}\right), \dot{y}_{2}\left(t, x_{0}\right)$ anulase para $t=\frac{p_{x_{0}}}{2}$.

Mas $\frac{p_{x_{0}}}{2}<\frac{4 \pi+1}{12}$ e isso contraria uma das conclusões $(i i)$ e $(i i i)$.

Gostaríamos de enfatizar as conclusões $(i),(i i)$ e (iii) que foram obtidas aqui. Elas decorrem apenas de $g(0)=1$, e valem sempre nessa situação (seja $g$ constante ou não). Faremos uso delas nas demonstrações a seguir várias vezes, em particular nas Proposições 5.1.3 e 5.1.4.

Em [BNdOCG02], para todo $x_{0}$ suficientemente pequeno próximo da origem são definidos os tempos de parada.

Considere $n=\sqrt{4 g(0) / f(0)}$,

$$
\begin{aligned}
& t_{1}\left(x_{0}\right)=n \text {-ésimo zero estritamente positivo de } \dot{y}_{1}\left(., x_{0}\right) . \\
& t_{2}\left(x_{0}\right)=n \text {-ésimo zero estritamente positivo de } y_{2}\left(., x_{0}\right) .
\end{aligned}
$$

Como estamos interessados somente no caso $g(0)=f(0)$, ou seja, $n=2$, iremos considerar:

Definição 5.1.1. Para todo $x_{0}$ suficientemente pequeno

$$
\begin{aligned}
& t_{1}\left(x_{0}\right)=2^{o} \text { zero estritamente positivo de } \dot{y}_{1}\left(., x_{0}\right) \\
& t_{2}\left(x_{0}\right)=2^{o} \text { zero estritamente positivo de } y_{2}\left(., x_{0}\right) .
\end{aligned}
$$

A próxima proposição nos permite relacionar a ocorrência do $\tau\left(x_{0}\right)$-isocronismo com as funções $t_{1}, t_{2}$ e $\tau$.

Proposição 5.1.3. Se há $\tau\left(x_{0}\right)$-isocronismo em (5.1), então existe $\delta>0$ tal que $t_{1}\left(x_{0}\right)=t_{2}\left(x_{0}\right)=$ $\tau\left(x_{0}\right)$, para todo $0 \leq x_{0}<\delta$.

Demonstração. Como o sistema possui $\tau\left(x_{0}\right)$-isocronismo, então $y_{1}\left(t, x_{0}\right)$ e $y_{2}\left(t, x_{0}\right)$ são periódicas de menor período $\tau\left(x_{0}\right)$.

Seja $\delta>0$ da prova da Proposição 5.1.2. Sabemos que, para todo $0 \leq x_{0}<\delta, \tau\left(x_{0}\right) \in(2 \pi-$ $\left.\frac{1}{2}, 2 \pi+\frac{1}{2}\right)$ e $\dot{y}_{1}\left(t, x_{0}\right), y_{2}\left(t, x_{0}\right)$ anulam-se em $\left(0,2 \pi+\frac{1}{2}\right)$ exatamente duas vezes, uma em $(\pi-1, \pi+1)$ e outra em $\left(2 \pi-\frac{1}{2}, 2 \pi+\frac{1}{2}\right)$. Concluímos na demonstração da Proposição 5.1.2 que o primeiro zero estritamente positivo de $\dot{y}_{1}\left(t, x_{0}\right)$ e $y_{2}\left(t, x_{0}\right)$ pertence ao intervalo $(\pi-1, \pi+1)$ e o segundo zero estritamente positivo percente a $\left(2 \pi-\frac{1}{2}, 2 \pi+\frac{1}{2}\right)$. Como $\tau\left(x_{0}\right) \in\left(2 \pi-\frac{1}{2}, 2 \pi+\frac{1}{2}\right)$ e, por periodicidade, $\dot{y}_{1}\left(\tau\left(x_{0}\right), x_{0}\right)=0$ e $y_{2}\left(\tau\left(x_{0}\right), x_{0}\right)=0$, concluí-se que $t_{1}\left(x_{0}\right)=t_{2}\left(x_{0}\right)=\tau\left(x_{0}\right)$.

Na proposição a seguir obtemos uma condição suficiente para ocorrência de $\tau\left(x_{0}\right)$-isocronismo.

Proposição 5.1.4. Se $g(0)=f(0)$ e $t_{1}\left(x_{0}\right)=t_{2}\left(x_{0}\right)=\tau\left(x_{0}\right)$, para $x_{0} \geq 0$, então (5.1) tem $\tau\left(x_{0}\right)$-isocronismo.

Demonstração. Suponha, como antes, $f(0)=1$. Assim, $g(0)=1$.

Sejam $y_{1}\left(t, x_{0}\right)$ e $y_{2}\left(t, x_{0}\right)$ as soluções normalizadas de $(5.1)$ e considere $\delta>0$ definido na demonstração da Proposição 5.1.2.

Pela propriedade do Wronskiano,

$$
y_{1}\left(t, x_{0}\right) \dot{y}_{2}\left(t, x_{0}\right)-\dot{y}_{1}\left(t, x_{0}\right) y_{2}\left(t, x_{0}\right)=1, \quad \forall t \in \mathbb{R} .
$$


Note ainda que, como observado após a Proposição 5.1.2, se $0 \leq x_{0}<\delta$ então, caso $y_{1}\left(t, x_{0}\right)$ e $y_{2}\left(t, x_{0}\right)$ seja periódicas, seu menor período terá de ser maior que $2 \pi-\frac{1}{2}$ e $\tau\left(x_{0}\right) \in I_{1}=$ $\left(2 \pi-\frac{1}{2}, 2 \pi+\frac{1}{2}\right)$.

Como $t_{1}\left(x_{0}\right)=t_{2}\left(x_{0}\right)=\tau\left(x_{0}\right)$, tem-se $\dot{y}_{1}\left(\tau\left(x_{0}\right), x_{0}\right)=y_{2}\left(\tau\left(x_{0}\right), x_{0}\right)=0$.

Agora vamos analisar a situação conforme o menor período de $g\left(x\left(t, x_{0}\right)\right)$ seja $\tau\left(x_{0}\right)$ ou $\frac{\tau\left(x_{0}\right)}{2}$.

Caso 1: Se o menor período de $g\left(x\left(t, x_{0}\right)\right)$ é $\tau\left(x_{0}\right)$. Então, pelo Teorema 5.1.1, $y_{1}\left(\tau\left(x_{0}\right), x_{0}\right)=$ $\dot{y}_{2}\left(\tau\left(x_{0}\right), x_{0}\right)$ e assim, por (5.4) com $t=\tau\left(x_{0}\right)$, tem-se $y_{1}\left(\tau\left(x_{0}\right), x_{0}\right)^{2}=\dot{y}_{2}\left(\tau\left(x_{0}\right), x_{0}\right)^{2}=1$. Como foi destacado na demonstração da Proposição 5.1.2, $y_{1}\left(t, x_{0}\right)>0$ em $I_{2}$, portanto $y_{1}\left(\tau\left(x_{0}\right), x_{0}\right)=1$ e, por (5.4), tem-se $\dot{y}_{2}\left(\tau\left(x_{0}\right), x_{0}\right)=1$.

Assim, $\tau\left(x_{0}\right)$ é um período tanto de $y_{1}\left(t, x_{0}\right)$ como de $y_{2}\left(t, x_{0}\right)$ e, como $\tau\left(x_{0}\right) \in I_{2}$, este é o menor período.

Caso 2: Se o menor período de $g\left(x\left(t, x_{0}\right)\right)$ é $p=\frac{\tau\left(x_{0}\right)}{2}$.

Como já comentado, nesse caso não há como $p$ ser menor período de $y_{1}\left(t, x_{0}\right)$ ou $y_{2}\left(t, x_{0}\right)$.

Por outro lado, o Teorema 5.1.1 garante que pelo menos uma dessas duas funções é periódica com menor período $p$ ou $2 p$. Resulta daqui que $y_{1}\left(t, x_{0}\right)$ ou $y_{2}\left(t, x_{0}\right)$ é periódica com menor período $2 p=\tau\left(x_{0}\right)$.

Isso mostra que $y_{1}\left(\tau\left(x_{0}\right), x_{0}\right)=1$ ou $\dot{y}_{2}\left(\tau\left(x_{0}\right), x_{0}\right)=1$.

Como $\dot{y}_{1}\left(\tau\left(x_{0}\right), x_{0}\right)=y_{2}\left(\tau\left(x_{0}\right), x_{0}\right)=0$, usa-se (5.4) e, da mesma forma como no caso $I$, concluise que ambas as funções $y_{1}\left(t, x_{0}\right)$ e $y_{2}\left(t, x_{0}\right)$ são periódicas de menor período $2 p=\tau\left(x_{0}\right)$.

Teorema 5.1.2. Se há $\tau\left(x_{0}\right)$-isocronismo em (5.1) e $f$ e g são de classe $C^{k}$, então

$$
\tau^{(j)}(0)=t_{1}^{(j)}(0)=t_{2}^{(j)}(0) \quad \text { para todo } \quad 0 \leq j \leq k .
$$

Além disso, se $f$ e g são funções analíticas (5.1), tem $\tau\left(x_{0}\right)$-isocronismo se, e somente se,

$$
\tau^{(k)}(0)=t_{1}^{(k)}(0)=t_{2}^{(k)}(0) \quad \text { para todo } \quad k \geq 0 .
$$

Demonstração. Da demonstração da Proposição (4.1.1), concluímos que as funções $t_{1}$ e $t_{2}$ pertencem à mesma classe de diferenciabilidade das funções $\dot{y}_{1}\left(. ; x_{0}\right)$ e $y_{2}\left(; x_{0}\right)$, como essas são de classe $C^{k}$, segue que $t_{1}$ e $t_{2}$ também são de classe $C^{k}$. O mesmo vale para a função período $\tau\left(x_{0}\right)$, como $f$ é $C^{k}$, então $\tau\left(x_{0}\right)$ também é de classe $C^{k}$. Um resultado análogo é válido quando $f$ e $g$ são funções analíticas. Disso, e da Proposição (5.1.3), segue que para $f$ e $g$ de classe $C^{k}$ ou analíticas, se o sistema possui $\tau\left(x_{0}\right)$-isocronismo, são satisfeitas, respectivamente, (5.5) e (5.6).

Para a condição suficiente, como $f$ e $g$ são analíticas, temos que $t_{1}\left(x_{0}\right), t_{2}\left(x_{0}\right)$ e $\tau\left(x_{0}\right)$ são analíticas, logo admitem expansão em série de Taylor em torno da origem que convergem para elas. Disso, e de (5.6) segue que $t_{1}\left(x_{0}\right)=t_{2}\left(x_{0}\right)=\tau\left(x_{0}\right)$. O resultado segue da proposição 5.1.4.

Do teorema acima, concluímos que se $f(x)$ é analítica, caso existam, as funções $g(x)$ analíticas tal que o sistema (5.1) possui $\tau\left(x_{0}\right)$-isocronismo são aquelas que a igualdade (5.6) é satisfeita. Por outro lado, se $f(x)$ é de classe $C^{k}$ as condições (5.5) não são suficientes para garantir a existência de funções $g(x)$ de classe $C^{k}$, de modo que ocorra $\tau\left(x_{0}\right)$-isocronismo, porém podemos garantir que se essas funções existirem elas são tais que satisfazem a igualdade (5.5).

Esse teorema será de extrema importância para demonstração dos resultados dos próximos capítulos. Em particular, no Capítulo 7, obtemos várias aplicações interessantes desse teorema para 
o caso $C^{k}$.

\subsection{Cálculo das derivadas de $\tau, t_{1}$ e $t_{2}$}

Esta seção é dedicada à apresentação de um método que permite calcular $\tau^{(k)}(0), t_{1}^{(k)}(0)$ e $t_{2}^{(k)}(0)$, para todo $k$ natural. Este método foi apresentado originalmente em [BNdOCG02], no qual os autores estudaram os casos em que $4 g(0)=n^{2} f(0)$, para algum $n \in \mathbb{N}$, e apresentaram as fórmulas das derivadas de $\tau, t_{1}$ e $t_{2}$ em zero até a segunda ordem.

Aqui, estudamos o nosso caso de interesse, ou seja, $n=2$. Calculamos explicitamente as fórmulas das derivadas de $\tau, t_{1}$ e $t_{2}$ em 0 até a quarta ordem e aplicando sucessivamente o método deduzimos expressões para $\tau^{(k)}(0), t_{1}^{(k)}(0)$ e $t_{2}^{(k)}(0)$, para todo $k$ natural.

No que segue, suporemos as funções $f$ e $g$ analíticas. As mesmas contas são válidas se as funções forem suaves.

\subsubsection{Equação das variações para $x$ e cálculo de $\tau^{(k)}(0)$}

Estamos interessados em calcular as derivadas $\tau^{k}(0), \forall k \in \mathbb{N}$.

Considere a igualdade

$$
\dot{x}\left(\tau\left(x_{0}\right), x_{0}\right)=0 \quad \text { e } \quad \tau(0)=2 \pi,
$$

válida para todo $x_{0}$ suficientemente pequeno.

Derivando (5.7) repetidamente com respeito à $x_{0}$ e fazendo $x_{0}=0$, obtemos expressões para o cálculo das derivadas de $\tau^{k}(0)$.

De fato, derivando (5.7) com respeito à $x_{0}$

$$
\ddot{x}\left(\tau\left(x_{0}\right) ; x_{0}\right) \tau^{\prime}\left(x_{0}\right)+\frac{\partial \dot{x}}{\partial x_{0}}\left(\tau\left(x_{0}\right) ; x_{0}\right)=0
$$

e fazendo $x_{0}=0$, temos

$$
\ddot{x}(2 \pi ; 0) \tau^{\prime}(0)+\frac{\partial \dot{x}}{\partial x_{0}}(2 \pi ; 0)=0 .
$$

Assim, obtemos uma expressão para o cálculo de $\tau^{\prime}(0)$ (se $\frac{\partial \dot{x}}{\partial x_{0}}(2 \pi ; 0)=0$, derivamos (5.8) novamente com respeito à $x_{0}$ e assim sucessivamente até ser possível calcular $\tau^{\prime}(0)$ ).

Agora, derivando (5.8) com respeito à $x_{0}$

$$
\dddot{x}\left(\tau\left(x_{0}\right) ; x_{0}\right)\left(\tau^{\prime}\left(x_{0}\right)\right)^{2}+2 \frac{\partial \ddot{x}}{\partial x_{0}}\left(\tau\left(x_{0}\right) ; x_{0}\right) \tau^{\prime}\left(x_{0}\right)+\ddot{x}\left(\tau\left(x_{0}\right) ; x_{0}\right) \tau^{\prime \prime}\left(x_{0}\right)+\frac{\partial^{2} \dot{x}}{\partial x_{0}^{2}}\left(\tau\left(x_{0}\right) ; x_{0}\right)=0 .
$$

Fazendo $x_{0}=0$ em (5.9) e usando o valor de $\tau^{\prime}(0)$, obtemos uma expressão para o cálculo de $\tau^{\prime \prime}(0)$.

Assim, de maneira recorrente, para todo $k \in \mathbb{N}$, fazendo $\frac{\partial^{k} \dot{x}}{\partial x_{0}^{k}}\left(\tau\left(x_{0}\right) ; x_{0}\right)=0$ e tomando $x_{0}=0$, obtemos uma expressão para o cálculo de $\tau^{(k)}(0)$.

Observe que para o cálculo de $\tau^{k}(0)$ precisamos conhecer as derivadas com respeito ao tempo 
das funções

$$
\frac{\partial^{p} x}{\partial x_{0}^{p}}\left(\tau\left(x_{0}\right) ; x_{0}\right), \quad \text { com } \quad 0 \leq p \leq k
$$

calculadas em $t=2 \pi$.

No que segue, iremos utilizar o método das equações variacionais para calcular estas funções.

Seja $x\left(t, x_{0}\right)$ a solução do problema de Cauchy

$$
\ddot{x}=-x f(x) \quad x(0)=x_{0}, \quad \dot{x}(0)=0 .
$$

Lembremos que como $f$ é uma função analítica, as funções $x\left(t ; x_{0}\right)$ e $\ddot{x}\left(t, x_{0}\right)$ também são analíticas com respeito a $\left(t ; x_{0}\right)$.

Derivando $x\left(t ; x_{0}\right)$ e $\ddot{x}\left(t, x_{0}\right)$ repetidamente com respeito à $x_{0}$ e tomando $x_{0}=0$, obtemos um sistema de equações diferenciais para as funções:

$$
\mu_{k}(t)=\frac{\partial^{k} x}{\partial x_{0}^{k}}(t ; 0)
$$

De fato, seja

$$
\mu_{k}\left(t ; x_{0}\right)=\frac{\partial^{k} x}{\partial x_{0}^{k}}\left(t ; x_{0}\right)
$$

Temos que

$$
\ddot{\mu_{k}}\left(t ; x_{0}\right)=\frac{\partial^{k} \ddot{x}}{\partial x_{0}^{k}}\left(t ; x_{0}\right)=\frac{\partial^{k}}{\partial x_{0}^{k}}\left(-x\left(t ; x_{0}\right) f\left(x\left(t ; x_{0}\right)\right)\right) .
$$

Aqui, $\ddot{\mu}_{k}\left(t ; x_{0}\right)=\frac{\partial^{2} \mu_{k}}{\partial t^{2}}\left(t ; x_{0}\right)$.

- $k=0$

$$
\ddot{\mu}_{0}\left(t ; x_{0}\right)=-\left(x\left(t, x_{0}\right) f\left(x\left(t ; x_{0}\right)\right)\right) .
$$

- $k=1$

$$
\begin{aligned}
\ddot{\mu}_{1}\left(t ; x_{0}\right) & =\frac{\partial}{\partial x_{0}}\left(-x\left(t, x_{0}\right) f\left(x\left(t ; x_{0}\right)\right)\right) \\
& =-\frac{\partial x}{\partial x_{0}}\left(t ; x_{0}\right) f\left(x\left(t ; x_{0}\right)\right)-x\left(t ; x_{0}\right) f^{\prime}\left(x\left(t ; x_{0}\right)\right) \frac{\partial x}{\partial x_{0}}\left(t ; x_{0}\right) .
\end{aligned}
$$

- $k=2$

$$
\begin{aligned}
\ddot{\mu}_{2}\left(t ; x_{0}\right) & =\frac{\partial^{2}}{\partial x_{0}^{2}}\left(-x\left(t, x_{0}\right) f\left(x\left(t ; x_{0}\right)\right)\right) \\
& =\frac{\partial}{\partial x_{0}}\left(\frac{\partial}{\partial x_{0}}\left(-x\left(t, x_{0}\right) f\left(x\left(t ; x_{0}\right)\right)\right)\right) \\
& =\frac{\partial}{\partial x_{0}}\left(\ddot{\mu}_{1}\left(t ; x_{0}\right)\right) \\
& =-\left(\frac{\partial^{2} x}{\partial x_{0}^{2}}\left(t ; x_{0}\right) f\left(x\left(t ; x_{0}\right)\right)+2\left(\frac{\partial x}{\partial x_{0}}\left(t ; x_{0}\right)\right)^{2} f^{\prime}\left(x\left(t ; x_{0}\right)\right)\right. \\
& \left.+x\left(t ; x_{0}\right) f^{\prime \prime}\left(x\left(t ; x_{0}\right)\right)\left(\frac{\partial x}{\partial x_{0}}\left(t ; x_{0}\right)\right)^{2}+x\left(t ; x_{0}\right) f^{\prime}\left(x\left(t ; x_{0}\right)\right) \frac{\partial^{2} x}{\partial x_{0}^{2}}\left(t ; x_{0}\right)\right)
\end{aligned}
$$


Em geral, para todo $k \in \mathbb{N}$, segue do Teorema de Schwarz que dado $\ddot{\mu}_{k-1}\left(t ; x_{0}\right)$ determinamos $\ddot{\mu_{k}}\left(t ; x_{0}\right)$ fazendo

$$
\ddot{\mu}_{k}\left(t ; x_{0}\right)=\frac{\partial}{\partial x_{0}}\left(\ddot{\mu}_{k-1}\left(t ; x_{0}\right)\right) .
$$

A expressão de $\ddot{\mu_{k}}\left(t ; x_{0}\right)$ é dada em função das derivadas da função $x\left(t ; x_{0}\right)$ com respeito à $x_{0}$ até a ordem $k$ e de derivadas de $f$ até a ordem $k$, calculadas em $x\left(t ; x_{0}\right)$.

Tomando $x_{0}=0$ nessas expressões, para cada natural $k$ obtemos uma equação diferencial de segunda ordem que $\mu_{k}(t ; 0)$ deve resolver.

Lembrando que $f(0)=1$ e denotando $\mu_{k}(t ; 0)$ por $\mu_{k}(t)$, temos com relação as condições iniciais dessa equação:

- para $k=0$ :

$$
\ddot{\mu}_{0}(t)+\mu_{0}(t) f\left(\mu_{0}(t)\right)=0, \quad \mu_{0}(0)=0, \quad \dot{\mu}_{0}(0)=0 .
$$

O problema de Cauchy não é linear, mas é trivialmente resolvido pela função nula. Usando que $\mu_{0}(t)=0$, segue que

- para $k=1$ :

$$
\ddot{\mu}_{1}(t)+\mu_{1}(t)=0, \quad \mu_{1}(0)=1, \quad \dot{\mu}_{1}(0)=0 .
$$

- para $k>1$ :

$$
\begin{gathered}
\ddot{\mu}_{k}(t)+\mu_{k}(t)=F^{k}\left[\mu_{1}(t), \cdots, \mu_{k-1}(t), f^{\prime}(0), f^{\prime \prime}(0), \cdots f^{(k-1)}(0)\right], \\
\mu_{k}(0)=0, \dot{\mu_{k}}(0)=0 .
\end{gathered}
$$

No lado direito da expressão do sistema não aparece $f^{(k)}(0)$, pois o termo $f^{(k)}(0), \forall k \in \mathbb{N}$, é múltiplo de $\mu_{0}(t)$ que é identicamente nulo.

Para ilustrar o caso $k>1$ seguem as equações para $k=2,3,4$

- $k=2$ :

$$
\ddot{\mu}_{2}(t)+\mu_{2}(t)=-2 f^{\prime}(0) \mu_{1}^{2}(t), \quad \mu_{2}(0)=0, \quad \dot{\mu}_{2}(0)=0 .
$$

- $k=3$ :

$$
\ddot{\mu}_{3}(t)+\mu_{3}(t)=-3 f^{\prime \prime}(0) \mu_{1}^{3}(t)-6 f^{\prime}(0) \mu_{1}(t) \mu_{2}(t), \quad \mu_{3}(0)=0, \quad \dot{\mu_{3}}(0)=0 .
$$

- $k=4$ :

$$
\begin{gathered}
\ddot{\mu}_{4}(t)+\mu_{4}(t)=-4 f^{\prime}(0) \mu_{1}(t) \mu_{3}(t)-6 \mu_{2}(t)\left[f^{\prime \prime}(0)\left(\mu_{1}(t)\right)^{2}+\mu_{2}(t) f^{\prime}(0)\right] \\
-4 \mu_{1}(t)\left[f^{(3)}(0)\left(\mu_{1}(t)\right)^{3}+3 \mu_{2}(t) \mu_{1}(t) f^{\prime \prime}(0)+\mu_{3}(t) f^{\prime}(0)\right] . \\
\mu_{4}(0)=0, \quad \dot{\mu}_{4}(0)=0
\end{gathered}
$$

Para $k=1$, a solução é

$$
\mu_{1}(t)=\cos (t) .
$$

Para $k>1$, os sistemas são triangulares lineares e podem ser resolvidos com funções elementares. 
Substituindo os valores de $\mu_{0}(t)$ e $\mu_{1}(t)$ na equação do sistema com $k=2$, obtemos

$$
\ddot{\mu}_{2}(t)+\mu_{2}(t)=-2 f^{\prime}(0) \cos ^{2}(t), \quad \mu_{2}(0)=0, \quad \dot{\mu_{2}}(0)=0,
$$

cuja solução é

$$
\mu_{2}(t)=\frac{f^{\prime}(0)}{3}(2 \cos (t)+2 \cos (2 t)-3) .
$$

Fazendo o mesmo processo para $k=3$ e $k=4$, temos

$$
\begin{aligned}
\mu_{3}(t)=\frac{1}{24} f^{\prime}(0)^{2}(29 \cos (t)+16 \cos (2 t)+3 \cos (3 t)+60 t \sin (t)-48) \\
-\frac{3}{16} f^{\prime \prime}(0)(6 t \sin (t)+\sin (2 t) \sin (t)) . \\
\mu_{4}(t)=-\frac{21}{2} f^{\prime \prime}(0) f^{\prime}(0) \cos (t)^{2}-\frac{5}{2} f^{\prime \prime \prime}(0)+6 f^{\prime}(0)^{3} \cos (t) \cos (2 t)-\frac{31}{36} f^{\prime}(0)^{3} \cos (t) \cos (3 t) \\
+\quad 10 f^{\prime}(0)^{3} \cos (t) t \sin (t)-\frac{113}{6} f^{\prime}(0)^{3}+\frac{38}{3} f^{\prime}(0)^{3} \cos (t)^{2}+\frac{29}{18} f^{\prime}(0)^{3} \cos (t) \\
-\frac{9}{2} f^{\prime}(0) \cos (t) f^{\prime \prime}(0) t \sin (t)+\frac{105}{8} f^{\prime \prime}(0) f^{\prime}(0)-\frac{9}{2} f^{\prime \prime}(0) f^{\prime}(0) t \sin (t)+10 f^{\prime}(0)^{3} t \sin (t) \\
-\frac{17}{8} \cos (t) f^{\prime \prime}(0) f^{\prime}(0)-\frac{1}{20} \cos (t) \cos (5 t) f^{\prime \prime \prime}(0)-\frac{1}{12} \cos (t) \cos (5 t) f^{\prime}(0)^{3} \\
-\frac{1}{2} \cos (t) \cos (4 t) f^{\prime}(0)^{3}-\frac{1}{4} \cos (t) \cos (3 t) f^{\prime \prime \prime}(0)+\ldots
\end{aligned}
$$

(Devido à extensão de $\mu_{4}(t)$, colocamos aqui apenas os primeiros termos de sua expressão, no Apêndice A a expressão está completa).

Assim, de maneira recorrente, conhecidas as soluções $\mu_{p}(t), 0 \leq p \leq k-1$, substituímos essas soluções no sistema

$$
\begin{gathered}
\ddot{\mu}_{k}(t)+\mu_{k}(t)=F^{k}\left[\mu_{0}(t), \mu_{1}(t), \ldots, \mu_{k-1}(t), f^{\prime}(0), f^{\prime \prime}(0), \ldots f^{(k-1)}(0)\right] \\
\mu_{k}(0)=0, \mu_{k}(0)=0
\end{gathered}
$$

e obtemos a solução

$$
\mu_{k}(t)=F_{1}^{k}\left[\text { funções elementares, } f^{\prime}(0) \ldots, f^{(k-1)}(0)\right]
$$

Voltemos ao cálculo das derivadas da função período. Temos que:

$$
\ddot{x}(2 \pi ; 0)) \tau^{\prime}(0)+\frac{\partial \dot{x}}{\partial x_{0}}(2 \pi ; 0)=0,
$$

está relação nos dá $0=0$. Então, derivando a expressão acima com respeito à $x_{0}$, obtém-se

$$
\dddot{x}\left(\tau\left(x_{0}\right) ; x_{0}\right)\left(\tau^{\prime}\left(x_{0}\right)\right)^{2}+2 \frac{\partial \ddot{x}}{\partial x_{0}}\left(\tau\left(x_{0}\right) ; x_{0}\right) \tau^{\prime}\left(x_{0}\right)+\ddot{x}\left(\tau\left(x_{0}\right) ; x_{0}\right) \tau^{\prime \prime}\left(x_{0}\right)+\frac{\partial^{2} \dot{x}}{\partial x_{0}^{2}}\left(\tau\left(x_{0}\right) ; x_{0}\right)=0 \text {. }
$$


Agora, tomando $x_{0}=0$ em (5.12),

$$
\dddot{x}(2 \pi, 0) \tau^{\prime}(0)^{2}+2 \frac{\partial \ddot{x}}{\partial x_{0}}(2 \pi, 0) \tau^{\prime}(0)+\ddot{x}(2 \pi, 0) \tau^{\prime \prime}(0)+\frac{\partial^{2} \dot{x}}{\partial x_{0}^{2}}(2 \pi, 0)=0,
$$

isto é,

$$
0+2 \ddot{\mu}_{1}(2 \pi) \tau^{\prime}(0)+0+\dot{\mu}_{2}(2 \pi)=0
$$

Como

$$
\ddot{\mu}_{1}(2 \pi)=-\cos (2 \pi)=-1 \neq 0,
$$

obtemos o valor de $\tau^{\prime}(0)$ :

$$
\tau^{\prime}(0)=\frac{\dot{\mu}_{2}(2 \pi)}{2}
$$

Usando a expressão de $\mu_{2}(t)$, temos que

$$
\tau^{\prime}(0)=0
$$

Tomando a derivada de (5.12) com respeito a $x_{0}$, fazendo $x_{0}=0$ e usando que $\tau^{\prime}(0)=0$, obtemos:

$$
3 \frac{\partial \ddot{x}}{\partial x_{0}}(2 \pi, 0) \tau^{\prime \prime}(0)+\ddot{x}(2 \pi, 0) \tau^{(3)}(0)+\frac{\partial^{3} \dot{x}}{\partial x_{0}^{3}}(2 \pi, 0)=0,
$$

isto é,

$$
3 \ddot{\mu}_{1}(2 \pi) \tau^{\prime \prime}(0)+\ddot{\mu}_{0}(2 \pi) \tau^{(3)}(0)+\dot{\mu}_{3}(2 \pi)=0
$$

Usando as fórmulas de $\mu_{0}(t), \mu_{1}(t)$ e $\mu_{3}(t)$ o valor de $\tau^{\prime \prime}(0)$ é:

$$
\tau^{\prime \prime}(0)=\frac{\dot{\mu}_{3}(2 \pi)}{3}=\pi\left(\frac{5}{3} f^{\prime}(0)^{2}-\frac{3}{4} f^{\prime \prime}(0)\right) .
$$

Repetindo o processo acima duas vezes, obtemos para $\tau^{(3)}(0)$ e $\tau^{(4)}(0)$ :

$$
\tau^{(3)}(0)=\frac{6 \ddot{\mu}_{2}(2 \pi) \tau^{\prime \prime}(0)+\dot{\mu}_{4}(2 \pi)}{4} ;
$$

$\mathrm{e}$

$$
\tau^{(4)}(0)=\frac{10 \ddot{\mu}_{3}(2 \pi) \tau^{\prime \prime}(0)+10 \ddot{\mu}_{2}(2 \pi) \tau^{(3)}(0)+\dot{\mu}_{5}(2 \pi)}{5} .
$$

Utilizando as expressões de $\mu_{2}(t), \mu_{3}(t), \mu_{5}(t), \tau^{\prime \prime}(0)$ e $\tau^{(3)}(0)$ :

$$
\begin{gathered}
\tau^{(3)}(0)=\pi\left(-\frac{3}{2} f^{\prime \prime}(0) f^{\prime}(0)+\frac{10}{3} f^{\prime}(0)^{3}\right) \\
\tau^{(4)}(0)=\pi\left(7 f^{\prime}(0) f^{\prime \prime \prime}(0)-\frac{275}{8} f^{\prime}(0)^{2} f^{\prime \prime}(0)+\frac{171}{64} f^{\prime \prime}(0)^{2}+\frac{385}{12} f^{\prime}(0)^{4}-\frac{5}{8} f^{(4)}(0)\right) .
\end{gathered}
$$

O cálculo de $\mu_{5}(t)$ está no Apêndice.

Seja

$$
r_{k}\left(\tau\left(x_{0}\right) ; x_{0}\right)=\frac{\partial^{k} \dot{x}}{\partial x_{0}^{k}}\left(\tau\left(x_{0}\right) ; x_{0}\right)
$$


temos que

$$
\begin{aligned}
r_{0}\left(\tau\left(x_{0}\right) ; x_{0}\right) & =\dot{x}\left(\tau\left(x_{0}\right) ; x_{0}\right) \\
r_{1}\left(\tau\left(x_{0}\right) ; x_{0}\right) & =\frac{\partial \dot{x}}{\partial x_{0}}\left(\tau\left(x_{0}\right) ; x_{0}\right)=\frac{\partial}{\partial x_{0}}\left(r_{0}\left(\tau\left(x_{0}\right) ; x_{0}\right)\right) ; \\
r_{2}\left(\tau\left(x_{0}\right) ; x_{0}\right) & =\frac{\partial^{2} \dot{x}}{\partial x_{0}^{2}}\left(\tau\left(x_{0}\right), x_{0}\right)=\frac{\partial}{\partial x_{0}}\left(r_{1}\left(\tau\left(x_{0}\right) ; x_{0}\right)\right) .
\end{aligned}
$$

Assim, para todo $k \in \mathbb{N}$, derivando $r_{k-1}\left(\tau\left(x_{0}\right) ; x_{0}\right)$ com respeito a $x_{0}$, obtemos $r_{k}\left(\tau\left(x_{0}\right) ; x_{0}\right)$.

Como mostramos anteriormente, igualando $r_{1}\left(\tau\left(x_{0}\right) ; x_{0}\right)$ a zero e fazendo $x_{0}=0$, obtemos $0=0$ (usando que $\tau(0)=2 \pi$ ).

Fazendo o mesmo para $r_{2}\left(\tau\left(x_{0}\right) ; x_{0}\right)$ achamos uma expressão para o cálculo de $\tau^{\prime}(0)$, e assim sucessivamente. Logo, fazendo $r_{k+1}\left(\tau\left(x_{0}\right), x_{0}\right)=0$ e tomando $x_{0}=0$ encontramos uma fórmula para o cálculo de $\tau^{(k)}(0)$.

Note que para $k>1$, ao aplicarmos a regra da cadeia, o termo $\tau^{(k)}(0)$ sempre será múltiplo de $\ddot{\mu}_{1}(2 \pi)$ que é não nulo. Logo, $\tau^{(k)}(0)$ está bem definido.

Usando que $\tau^{\prime}(0)=0$ e $\tau(0)=2 \pi$, da Regra da Cadeia para funções de duas variáveis, segue que a expressão de $\tau^{(k)}(0)$ pode ser escrita da seguinte maneira:

$$
\tau^{(k)}(0)=\frac{1}{k+1} \dot{\mu}_{k+1}(2 \pi)+G^{k}\left[\tau^{(2)}(0), \ldots, \tau^{(2 k-1)}(0), \ddot{\mu_{2}}(2 \pi), \ldots, \ddot{\mu}_{k-1}(2 \pi)\right] .
$$

\subsubsection{Equação das variações para $y_{1}$ e cálculo $t_{1}^{k}(0)$}

A equação abaixo é satisfeita implicitamente por $t_{1}\left(x_{0}\right)$ para $x_{0}$ perto de zero:

$$
\dot{y}_{1}\left(t_{1}\left(x_{0}\right) ; x_{0}\right)=0 \quad \text { e } \quad t_{1}(0)=2 \pi \text {. }
$$

Derivando (5.14) repetidamente com respeito à $x_{0}$ e fazendo $x_{0}=0$, obtemos expressões para o cálculo das derivadas de $t_{1}^{k}(0)$.

De fato, derivando (5.14) com respeito à $x_{0}$, temos

$$
\ddot{y}_{1}\left(t_{1}\left(x_{0}\right) ; x_{0}\right) t_{1}^{\prime}\left(x_{0}\right)+\frac{\partial \dot{y}_{1}}{\partial x_{0}}\left(t_{1}\left(x_{0}\right) ; x_{0}\right)=0 .
$$

E fazendo $x_{0}=0$,

$$
\ddot{y}_{1}(2 \pi ; 0) t_{1}^{\prime}(0)+\frac{\partial \dot{y}_{1}}{\partial x_{0}}(2 \pi ; 0)=0 .
$$

Assim, obtemos uma expressão para o cálculo de $t_{1}^{\prime}(0)$.

Derivando (5.15) com respeito à $x_{0}$,

$$
\begin{aligned}
& \dddot{y}_{1}\left(t_{1}\left(x_{0}\right), x_{0}\right) t_{1}^{\prime}\left(x_{0}\right)^{2}+2 \frac{\partial \ddot{y}_{1}}{\partial x_{0}}\left(t_{1}\left(x_{0}\right) ; x_{0}\right) t_{1}^{\prime}\left(x_{0}\right) \\
& +\ddot{y}_{1}\left(t_{1}\left(x_{0}\right) ; x_{0}\right) t_{1}^{\prime \prime}\left(x_{0}\right)+\frac{\partial^{2} \dot{y}_{1}}{\partial x_{0}^{2}}\left(t_{1}\left(x_{0}\right) ; x_{0}\right)=0
\end{aligned}
$$


fazendo $x_{0}=0$ e usando $t_{1}^{\prime}(0)$,

$$
\begin{aligned}
& \dddot{y}_{1}(2 \pi, 0) t^{\prime}(0)^{2}+2 \frac{\partial \ddot{y}_{1}}{\partial x_{0}}(2 \pi ; 0) t_{1}^{\prime}(0) \\
& +\ddot{y}_{1}(2 \pi, 0) t_{1}^{\prime \prime}(0)+\frac{\partial^{2} \dot{y}_{1}}{\partial x_{0}^{2}}(2 \pi ; 0)=0 .
\end{aligned}
$$

Obtemos uma expressão para o cálculo de $t_{1}^{\prime \prime}(0)$.

Assim, de maneira recorrente, para todo $k \in \mathbb{N}$, fazendo $\frac{\partial^{k} \dot{y}_{1}}{\partial x_{0}^{k}}\left(t_{1}\left(x_{0}\right), x_{0}\right)=0$ e tomando $x_{0}=0$, obtemos uma expressão para o cálculo de $t_{1}^{(k)}(0)$.

Observe que para o calcular $t_{1}^{(k)}(0)$ precisamos conhecer as derivadas com respeito ao tempo das funções

$$
\frac{\partial^{p} y_{1}}{\partial x_{0}^{p}}\left(\tau\left(x_{0}\right), x_{0}\right), \quad \text { com } \quad 0 \leq p \leq k
$$

calculadas em $t=2 \pi$.

No que segue, iremos utilizar o método das equações variacionais para calcular estas funções.

A função $t \mapsto y_{1}\left(t ; x_{0}\right)$ é solução do Problema de Cauchy

$$
\ddot{y_{1}}=-g\left(x\left(t ; x_{0}\right)\right) y_{1}, \quad y_{1}(0)=1, \quad \dot{y}_{1}(0)=0,
$$

Derivando $\ddot{y_{1}}=-g\left(x\left(t ; x_{0}\right)\right) y_{1}$ com respeito à $x_{0}$ e fazendo $x_{0}=0$, obtemos sistemas de equações para as funções desconhecidas

$$
v_{k}(t)=\frac{\partial^{k} y_{1}}{\partial x_{0}^{k}}(t ; 0)
$$

contendo também as funções $\mu_{k}(t, 0)$.

De fato, seja

$$
v_{k}\left(t ; x_{0}\right)=\frac{\partial^{k} y_{1}}{\partial x_{0}^{k}}\left(t ; x_{0}\right)
$$

Temos que

$$
\ddot{v_{k}}\left(t ; x_{0}\right)=\frac{\partial^{k} \ddot{y}_{1}}{\partial x_{0}^{k}}\left(t ; x_{0}\right)=\frac{\partial^{k}}{\partial x_{0}^{k}}\left(-g\left(x\left(t ; x_{0}\right)\right) y_{1}\left(t ; x_{0}\right)\right) .
$$

Aqui, $\ddot{v_{k}}\left(t ; x_{0}\right)=\frac{\partial^{2} v_{k}}{\partial t^{2}}\left(t ; x_{0}\right)$.

- Para $k=0$ :

$$
\ddot{v}_{0}\left(t ; x_{0}\right)=-g\left(x\left(t ; x_{0}\right)\right) y_{1}\left(t ; x_{0}\right) .
$$

- Para $k=1$ :

$$
\begin{aligned}
\ddot{v}_{1}\left(t ; x_{0}\right) & \left.=\frac{\partial}{\partial x_{0}}\left(-g\left(x\left(t ; x_{0}\right)\right) y_{1}\left(t ; x_{0}\right)\right)\right)=\frac{\partial}{\partial x_{0}}\left(\ddot{v}_{0}\left(t ; x_{0}\right)\right) \\
& =-\frac{\partial y_{1}}{\partial x_{0}}\left(t ; x_{0}\right) g\left(x\left(t ; x_{0}\right)\right)-y_{1}\left(t ; x_{0}\right) \frac{\partial x}{\partial x_{0}}\left(t ; x_{0}\right) g^{\prime}\left(x\left(t ; x_{0}\right)\right) .
\end{aligned}
$$


- Para $k=2$ :

$$
\begin{aligned}
\ddot{v}_{2}\left(t ; x_{0}\right) & =\frac{\partial^{2}}{\partial x_{0}^{2}}\left(-g\left(x\left(t ; x_{0}\right)\right) y_{1}\left(t ; x_{0}\right)\right) \\
& =-\frac{\partial}{\partial x_{0}}\left(\frac{\partial}{\partial x_{0}}\left(-g\left(x\left(t ; x_{0}\right)\right) y_{1}\left(t ; x_{0}\right)\right)\right) \\
& =-\frac{\partial}{\partial x_{0}}\left(\ddot{v}_{1}\left(t ; x_{0}\right)\right) \\
& =-\frac{\partial^{2} y_{1}}{\partial x_{0}^{2}}\left(t ; x_{0}\right) g\left(x\left(t ; x_{0}\right)\right)-2 \frac{\partial y_{1}}{\partial x_{0}}\left(t ; x_{0}\right) \frac{\partial x}{\partial x_{0}}\left(t ; x_{0}\right) g^{\prime}\left(x\left(t ; x_{0}\right)\right) \\
& -y_{1}\left(t ; x_{0}\right) \frac{\partial^{2} x}{\partial x_{0}^{2}}\left(t, x_{0}\right) g^{\prime}\left(x\left(t ; x_{0}\right)\right)-y_{1}\left(t ; x_{0}\right)\left(\frac{\partial x}{\partial x_{0}}\left(t ; x_{0}\right)\right)^{2} g^{\prime \prime}\left(x\left(t ; x_{0}\right)\right) .
\end{aligned}
$$

Em geral, para $k \in \mathbb{N}$, segue do Teorema de Schwarz que dado $\ddot{v}_{k-1}\left(t ; x_{0}\right)$ determinamos $\ddot{v}_{k}\left(t ; x_{0}\right)$ fazendo

$$
\ddot{v_{k}}\left(t ; x_{0}\right)=\frac{\partial}{\partial x_{0}}\left(v_{k-1}\left(t ; x_{0}\right)\right) .
$$

A expressão de $\ddot{v}_{k}\left(t ; x_{0}\right)$ será dada em função das derivadas parciais de $x\left(t ; x_{0}\right)$ e $y_{1}\left(t ; x_{0}\right)$ com respeito a $x_{0}$ até a ordem $k$ e de derivadas de $g$ até a ordem $k$ calculadas em $x\left(t ; x_{0}\right)$.

Tomando $x_{0}=0$ nessas expressões, para cada $k$ natural, obtemos uma equação diferencial de segunda ordem que $v_{k}(t, 0)$ deve resolver.

Lembrando que $f(0)=g(0)=1, \mu_{0}(t)=0$ e denotando $v_{k}(t, 0)$ por $v_{k}(t)$ temos, com relação as condições iniciais dessa equação:

- Para $k=0$ :

$$
\ddot{v}_{0}(t)+v_{0}(t)=0 \quad v_{0}(0)=1 \quad \dot{v}_{0}(0)=0
$$

- Para $k \neq 0$ :

$$
\begin{gathered}
\ddot{v}_{k}+v_{k}=F^{k}\left[\mu_{1}(t), \ldots, \mu_{k}(t), v_{0}(t), \ldots, v_{k-1}(t), g^{\prime}(0), \ldots, g^{(k)}(0)\right], \\
v_{k}(0)=0, \quad \dot{v}_{k}(0)=0 .
\end{gathered}
$$

Para ilustrar o caso $k>0$, seguem as equações para $k=1,2,3,4$.

- $k=1$ :

$$
\ddot{v}_{1}(t)+v_{1}(t)=-v_{0}(t) \mu_{1}(t) g^{\prime}(0) \quad v_{1}(0)=0 \quad \dot{v}_{1}(0)=0 .
$$

- $k=2$ :

$$
\begin{gathered}
\ddot{v}_{2}(t)+v_{2}(t)=-g^{\prime}(0) \mu_{2}(t) v_{0}(t)-2 g^{\prime}(0) \mu_{1}(t) v_{1}(t)-g^{\prime \prime}(0) \mu_{1}(t)^{2} v_{0}(t) \\
v_{2}(0)=0 \quad \dot{v}_{2}(0)=0 .
\end{gathered}
$$

- $k=3$ :

$$
\begin{aligned}
\ddot{v}_{3}(t)+v_{3}(t) & =-3 v_{2}(t) \mu_{1}(t) g^{\prime}(0)-3 v_{1}(t)\left(\mu_{1}^{2}(t) g^{\prime \prime}(0)+\mu_{2}(t) g^{\prime}(0)\right) \\
& -v_{0}(t)\left(\mu_{1}^{3}(t) g^{\prime \prime \prime}(0)+3 \mu_{2}(t) \mu_{1}(t) g^{\prime \prime}(0)+\mu_{3}(t) g^{\prime}(0)\right)
\end{aligned}
$$




$$
v_{3}(0)=0 \quad \dot{v}_{3}(0)=0
$$

- $k=4$ :

$$
\begin{gathered}
\ddot{v}_{4}(t)+v_{4}(t)=-4 \mu_{1}(t) v_{3}(t) g^{\prime}(0)-6 v_{2}(t)\left[g^{\prime \prime}(0)\left(\mu_{1}(t)\right)^{2}+\mu_{2}(t) g^{\prime}(0)\right] \\
-4 v_{1}(t)\left[\left(\mu_{1}(t)\right)^{3} g^{(3)}(0)+3 \mu_{2}(t) \mu_{1}(t) g^{\prime \prime}(0)+\mu_{3}(t) g^{\prime}(0)\right] \\
-\quad v_{0}(t)\left[\left(\mu_{1}(t)\right)^{4} g^{(4)}(0)+6 \mu_{2}(t)\left(\mu_{1}(t)\right)^{2} g^{(3)}(0)\right. \\
\left.+\quad 4 \mu_{3}(t) \mu_{1}(t) g^{\prime \prime}(0)+3\left(\mu_{2}(t)\right)^{2} g^{\prime \prime}(0)+\mu_{4}(t) g^{\prime}(0)\right] . \\
v_{4}(0)=0 \quad \dot{v}_{4}(0)=0 .
\end{gathered}
$$

Os sistemas são triangulares lineares e podem ser resolvidos por funções elementares.Para $k=$ $0, v_{0}(t)=\cos (t)$.

Substituindo $v_{0}(t)$ e $\mu_{1}(t)$ no sistema para $k=1$, temos

$$
\ddot{v}_{1}(t)+v_{1}(t)=-\cos ^{2}(t) g^{\prime}(0) \quad v_{1}(0)=0 \quad \dot{v}_{1}(0)=0 .
$$

cuja solução é

$$
v_{1}(t)=\frac{2 g^{\prime}(0)}{3}\left(\cos (t) \sin ^{2}\left(\frac{t}{2}\right)-\sin ^{2}(t)\right) .
$$

Substituindo os valores de $\mu_{1}(t), \mu_{2}(t), v_{0}(t)$ e $v_{1}(t)$ no sistema para $k=3$, e resolvendo esse sistema obtemos

$$
\begin{aligned}
v_{2}(t) & =2(-48+29 \cos (t)+16 \cos (2 t)+3 \cos (3 t)+60 t \sin (t)) f^{\prime}(0) g^{\prime}(0) \\
& +2(-48+29 \cos (t)+16 \cos (2 t)+3 \cos (3 t)+60 t \sin (t)) g^{\prime}(0)^{2} \\
& -18 \sin (t)(6 t+\sin (2 t)) g^{\prime \prime}(0)
\end{aligned}
$$

Os cálculos de $v_{3}(t)$ e $v_{4}(t)$ estão no Apêndice A.

Assim, de maneira recorrente, conhecidas as soluções $\mu_{p}$ e $v_{p-1}(t), 0 \leq p \leq k$, obtemos a solução $v_{k}(t)$.

Note que a expressão de $v_{k}(t)$ será da forma

$$
v_{k}(t)=F_{1}^{k}\left[\text { funções elementares, } f^{\prime}(0), \ldots, f^{(k-1)}(0), g^{\prime}(0), \ldots, g^{(k)}(0) .\right]
$$

Voltemos ao cálculo das derivadas de $t_{1}\left(x_{0}\right)$. Temos que

$$
\ddot{y}_{1}\left(t_{1}\left(x_{0}\right) ; x_{0}\right) t_{1}^{\prime}\left(x_{0}\right)+\frac{\partial \dot{y}_{1}}{\partial x_{0}}\left(t_{1}\left(x_{0}\right) ; x_{0}\right)=0,
$$

tomando $x_{0}=0$ em (5.17),

$$
\ddot{v}_{0}(2 \pi) t_{1}^{\prime}(0)+\dot{v}_{1}(2 \pi)=0 .
$$

Usando as expressões de $v_{0}$ e $v_{1}, \ddot{v}_{0}(2 \pi)=-1 \neq 0$ e

$$
t_{1}^{\prime}(0)=0 \text {. }
$$


Derivando (5.17) com respeito à $x_{0}$

$$
\begin{aligned}
& \dddot{y}_{1}\left(t_{1}\left(x_{0}\right), x_{0}\right) t^{\prime}\left(x_{0}\right)^{2}+2 \frac{\partial \ddot{y}_{1}}{\partial x_{0}}\left(t_{1}\left(x_{0}\right) ; x_{0}\right) t_{1}^{\prime}\left(x_{0}\right) \\
& +\ddot{y}_{1}\left(t_{1}\left(x_{0}\right) ; x_{0}\right) \tau^{\prime \prime}\left(x_{0}\right)+\frac{\partial^{2} \dot{y}_{1}}{\partial x_{0}^{2}}\left(t_{1}\left(x_{0}\right) ; x_{0}\right)=0
\end{aligned}
$$

e fazendo $x_{0}=0$, temos

$$
\dddot{v}_{0}(2 \pi) t_{1}^{\prime}(0)^{2}+2 \ddot{v}_{1}(2 \pi) t_{1}^{\prime}(0)+\ddot{v}_{0}(2 \pi) t_{1}^{\prime \prime}(0)+\dot{v}_{2}(2 \pi)=0
$$

Como $t_{1}^{\prime}(0)=0$, usando as expressões de $v_{0}(t)$ e $v_{2}(t)$, temos:

$$
t_{1}^{\prime \prime}(0)=\frac{\pi}{12}\left(10 f^{\prime}(0) g^{\prime}(0)+10 g^{\prime}(0)^{2}-9 g^{\prime \prime}(0)\right) \text {. }
$$

Repetindo o processo acima duas vezes, obtemos para $t_{1}^{(3)}(0)$ e $t_{1}^{(4)}(0)$ :

$$
t_{1}^{(3)}(0)=\frac{-\dot{v}_{3}(2 \pi)-3 \ddot{v}_{1}(2 \pi) t_{1}^{\prime \prime}(0)}{\ddot{v}_{0}(2 \pi)}
$$

e

$$
t_{1}^{(4)}(0)=\frac{-\dot{v}_{4}(2 \pi)-6 v_{2}(\ddot{2} \pi) t_{1}^{\prime \prime}(0)-4 \ddot{v}_{1}(2 \pi) \tau^{(3)}(0)}{\ddot{v}_{0}(2 \pi)}
$$

Utilizando as expressões de $v_{0}(t), v_{1}(t), v_{3}(t), v_{4}(t), t_{1}^{\prime \prime}(0)$ e $t_{1}^{(3)}(0)$, obtemos

$$
\begin{aligned}
t_{1}^{(3)}(0)= & \pi\left(\frac{5}{6} g^{\prime}(0)^{2} f^{\prime}(0)-\frac{5}{6} g^{\prime}(0)^{3}+\frac{3}{4} g^{\prime}(0) g^{\prime \prime}(0)-\frac{3}{4} g^{\prime}(0) f^{\prime \prime}(0)-\frac{3}{2} g^{\prime \prime}(0) f^{\prime}(0)\right. \\
+ & \left.\frac{10}{3} g^{\prime}(0) f^{\prime}(0)^{2}\right) . \\
t_{1}^{(4)}(0)= & \pi\left(-\frac{5}{8} g^{(4)}(0)+\frac{249}{64} g^{\prime \prime}(0)^{2}-\frac{39}{32} g^{\prime \prime}(0) f^{\prime \prime}(0)-\frac{575}{48} g^{\prime}(0) g^{\prime \prime}(0) f^{\prime}(0)\right. \\
& -\frac{157}{16} g^{\prime}(0) f^{\prime \prime}(0) f^{\prime}(0)+\frac{259}{48} g^{\prime}(0)^{4}+\frac{7}{3} g^{\prime}(0) g^{\prime \prime \prime}(0)+\frac{7}{2} g^{\prime \prime \prime}(0) f^{\prime}(0) \\
& +\frac{7}{6} g^{\prime}(0) f^{\prime \prime \prime}(0)-\frac{91}{24} g^{\prime \prime}(0) f^{\prime}(0)^{2}+\frac{223}{12} g^{\prime}(0) f^{\prime}(0)^{3}+\frac{43}{16} g^{\prime}(0)^{2} f^{\prime \prime}(0) \\
& \left.+\frac{89}{8} g^{\prime}(0)^{3} f^{\prime}(0)-\frac{145}{48} g^{\prime}(0)^{2} f^{\prime}(0)^{2}-\frac{551}{48} g^{\prime}(0)^{2} g^{\prime \prime}(0)\right) .
\end{aligned}
$$

Seja

$$
s_{k}\left(t_{1}\left(x_{0}\right) ; x_{0}\right)=\frac{\partial^{k} \dot{y}_{1}}{\partial x_{0}^{k}}\left(t_{1}\left(x_{0}\right) ; x_{0}\right),
$$

Para todo $k \in \mathbb{N}$, derivando $s_{k-1}\left(t_{1}\left(x_{0}\right) ; x_{0}\right)$ com respeito à $x_{0}$, obtemos $s_{k}\left(t_{1}\left(x_{0}\right) ; x_{0}\right)$.

Fazendo $s_{k}\left(t_{1}\left(x_{0}\right) ; x_{0}\right)=0$ e tomando $x_{0}=0$ encontramos uma fórmula para o cálculo de $t_{1}^{(k)}(0)$.

Note que para todo natural $k$, ao aplicarmos a Regra da Cadeia em (5.14), o termo $t_{1}^{(k)}(0)$ sempre será múltiplo de $\ddot{v}_{0}(2 \pi)$ que é não nulo. Logo, $t_{1}^{(k)}(0)$ está bem definido.

Usando que $t_{1}^{\prime}(0)=0$ e $t_{1}(0)=2 \pi$, da Regra da Cadeia para funções de duas variáveis segue 
que a expressão de $t_{1}^{k}(0)$ pode ser escrita da seguinte maneira:

$$
t_{1}^{(k)}(0)=\dot{v}_{k}(2 \pi)+G_{1}^{k}\left[t_{1}^{(2)}(0), \ldots, t_{1}^{(k-1)}(0), \ddot{v}_{1}(2 \pi), \ldots, \ddot{v}_{k-1}(2 \pi)\right] .
$$

\subsubsection{Equação das variações para $y_{2}$ e cálculo de $t_{2}^{(k)}(0)$}

A equação abaixo é satisfeita implicitamente por $t_{2}\left(x_{0}\right)$ para $x_{0}$ perto de zero:

$$
y_{2}\left(t_{2}\left(x_{0}\right) ; x_{0}\right)=0 \quad \text { e } \quad t_{2}(0)=2 \pi .
$$

Derivando (5.19) repetidamente com respeito à $x_{0}$ e fazendo $x_{0}=0$, obtemos expressões para $t_{2}^{(k)}(0)$.

De fato, derivando (5.19) com respeito à $x_{0}$

$$
\dot{y}_{2}\left(t_{2}\left(x_{0}\right) ; x_{0}\right) t_{2}^{\prime}\left(x_{0}\right)+\frac{\partial y_{2}}{\partial x_{0}}\left(t_{2}\left(x_{0}\right) ; x_{0}\right)=0
$$

e fazendo $x_{0}=0$

$$
\dot{y}_{2}(2 \pi ; 0) t_{2}^{\prime}(0)+\frac{\partial y_{2}}{\partial x_{0}}(2 \pi ; 0)=0 .
$$

Assim, obtemos uma expressão para o cálculo de $t_{2}^{\prime}(0)$.

Agora, derivando (5.20) com respeito à $x_{0}$, temos

$$
\begin{aligned}
& \ddot{y}_{2}\left(t_{2}\left(x_{0}\right), x_{0}\right) t_{2}^{\prime}\left(x_{0}\right)^{2}+2 \frac{\partial \dot{y}_{2}}{\partial x_{0}}\left(t_{2}\left(x_{0}\right) ; x_{0}\right) t_{2}^{\prime}\left(x_{0}\right) \\
& +\dot{y}_{2}\left(t_{2}\left(x_{0}\right) ; x_{0}\right) t_{2}^{\prime \prime}\left(x_{0}\right)+\frac{\partial^{2} y_{2}}{\partial x_{0}^{2}}\left(t_{2}\left(x_{0}\right) ; x_{0}\right)=0
\end{aligned}
$$

e fazendo $x_{0}=0$, temos

$$
\begin{aligned}
& \ddot{y}_{2}(2 \pi, 0) t_{2}^{\prime}(0)^{2}+2 \frac{\partial \dot{y}_{2}}{\partial x_{0}}(2 \pi ; 0) t_{2}^{\prime}(0) \\
& +\dot{y}_{2}(2 \pi ; 0) t_{2}^{\prime \prime}(0)+\frac{\partial^{2} y_{2}}{\partial x_{0}^{2}}(2 \pi ; 0)=0 .
\end{aligned}
$$

Usando $t_{2}^{\prime}(0)$, obtemos uma fórmula para o cálculo de $t_{2}^{\prime \prime}(0)$.

Assim, de maneira recorrente, para todo $k \in \mathbb{N}$, fazendo $\frac{\partial^{k} y_{2}}{\partial x_{0}^{k}}\left(t_{2}\left(x_{0}\right), x_{0}\right)=0$ e tomando $x_{0}=0$, obtemos uma expressão para o cálculo de $t_{2}^{(k)}(0)$.

Observe que para calcular $t_{2}^{(k)}(0)$, precisamos conhecer as derivadas com respeito ao tempo das funções

$$
\frac{\partial^{p} y_{2}}{\partial x_{0}^{p}}\left(t_{2}\left(x_{0}\right), x_{0}\right), \quad \text { com } \quad 0 \leq p \leq k
$$

calculadas em $t=2 \pi$.

No que segue, iremos utilizar o método das equações varicionais para calcular estas funções.

A função $t \mapsto y_{1}\left(t ; x_{0}\right)$ é solução do Problema de Cauchy

$$
\ddot{y}_{2}=-g\left(x\left(t ; x_{0}\right)\right) y_{2}, \quad y_{2}(0)=1, \quad \dot{y}_{2}(0)=0 .
$$

Derivando $\ddot{y}_{2}=-g\left(x\left(t ; x_{0}\right)\right) y_{2}$ com respeito à $x_{0}$ e fazendo $x_{0}=0$, obtemos sistemas de equações 
diferenciais para as funções desconhecidas

$$
u_{k}(t):=\frac{\partial^{k} y_{2}}{\partial x_{0}^{k}}(t ; 0)
$$

contendo também as funções $\mu_{k}(t)$.

Note que, para todo $k$ natural, a equação diferencial de segunda ordem cuja $u_{k}$ é solução é análoga a equação diferencial de segunda ordem cuja $v_{k}$ é solução, trocando $v_{k}$ por $u_{k}$ e mudando as condições iniciais do sistema para $u_{0}(t)$ por $u_{0}(0)=0, \dot{u}_{0}(0)=1$.

Assim, para

- $k=0$ :

$$
\ddot{u}_{0}(t)+u_{0}(t)=0 \quad u_{0}(0)=0 \quad \dot{u}_{0}(0)=1 ;
$$

- $k \neq 0$ :

$$
\begin{gathered}
\ddot{u}_{k}+u_{k}=F^{k}\left[\mu_{1}(t), \ldots, \mu_{k}(t), u_{0}(t), \ldots, u_{k-1}(t) ; g^{\prime}(0), \ldots, g^{(k)}(0)\right] . \\
u_{k}(0)=0, \quad \dot{u}_{k}(0)=0 .
\end{gathered}
$$

Para $k=0$, a solução do sistema é $u_{0}(t)=\sin (t)$.

Substituindo $u_{0}(t)$ e $\mu_{1}(t)$ na equação do sistema com $k=1$, temos

$$
\ddot{u}_{1}(t)+u_{1}(t)=-\cos (t) \sin (t) g^{\prime}(0) \quad u_{1}(0)=0 \quad \dot{u}_{1}(0)=0 .
$$

cuja solução é

$$
u_{1}(t)=\frac{2 g^{\prime}(0)}{3}\left(\cos (t) \sin (t)-\sin (t) \cos ^{2}\left(\frac{t}{2}\right)\right)
$$

Agora, substituindo $u_{0}(t), u_{1}(t), \mu_{1}(t)$ e $\mu_{2}(t)$ na equação do sistema com $k=2$, obtemos

$$
\begin{aligned}
288 u_{2}(t) & =(-168 t \cos (t)+86 \sin (t)+32 \sin (2 t)+6 \sin (3 t)) f^{\prime}(0) g^{\prime}(0) \\
& +(24 t \cos (t)+22 \sin (t)-32 \sin (2 t)+6 \sin (3 t)) g^{\prime}(0)^{2} \\
& +9(4 t \cos (t)-t \sin (t)+\sin (3 t)) g^{\prime \prime}(0)
\end{aligned}
$$

Assim, de maneira recorrente, conhecidas as $\mu_{p}$ e $u_{p-1}(t)$ soluções, $0 \leq p \leq k$, obtemos a solução $u_{k}(t)$. A expressão de $u_{k}(t)$ será da forma

$$
u_{k}(t)=F_{1}^{k}\left[\text { funções elementares, } f^{\prime}(0), \ldots, f^{(k-1)}(0), g^{\prime}(0), \ldots, g^{(k)}(0)\right] \text {. }
$$

Voltemos ao cálculo de $t_{2}^{(k)}(0)$. Temos que:

$$
\dot{y}_{2}\left(t_{2}\left(x_{0}\right) ; x_{0}\right) t_{2}^{\prime}\left(x_{0}\right)+\frac{\partial y_{2}}{\partial x_{0}}\left(t_{2}\left(x_{0}\right) ; x_{0}\right)=0,
$$

tomando $x_{0}=0$ :

$$
\dot{u}_{0}(2 \pi) t_{2}^{\prime}(0)+u_{1}(2 \pi)=0 .
$$

Como $\dot{u}_{0}(2 \pi)=\cos (2 \pi)=1 \neq 0$,

$$
t_{2}^{\prime}(0)=-u_{1}(2 \pi)
$$


Usando $u_{1}(t)$, temos

$$
t_{2}^{\prime}(0)=0
$$

Agora, derivando (5.21) com respeito a $x_{0}$

$$
\begin{aligned}
& \ddot{y}_{2}\left(t_{2}\left(x_{0}\right), x_{0}\right) t_{2}^{\prime}\left(x_{0}\right)^{2}+2 \frac{\partial \dot{y}_{2}}{\partial x_{0}}\left(t_{2}\left(x_{0}\right) ; x_{0}\right) t_{2}^{\prime}\left(x_{0}\right) \\
& +\dot{y}_{2}\left(t_{2}\left(x_{0}\right) ; x_{0}\right) t_{2}^{\prime \prime}\left(x_{0}\right)+\frac{\partial^{2} y_{2}}{\partial x_{0}^{2}}\left(t_{2}\left(x_{0}\right) ; x_{0}\right)=0,
\end{aligned}
$$

e fazendo $x_{0}=0$, temos

$$
\ddot{u}_{0}(2 \pi) t_{2}^{\prime}(0)+2 \dot{u}_{1}(2 \pi) t_{2}^{\prime}(0)+\dot{u}_{0}(2 \pi) t_{2}^{\prime \prime}(0)+u_{2}(2 \pi)=0 .
$$

Como $t_{2}^{\prime}(0)=0$ e $\dot{u}_{0}(2 \pi)=1$

$$
t_{2}^{\prime \prime}(0)=-u_{2}(2 \pi)
$$

Usando $u_{2}(t)$,

$$
t_{2}^{\prime \prime}(0)=\frac{\pi}{12}\left(14 f^{\prime}(0) g^{\prime}(0)-2 g^{\prime}(0)^{2}-3 g^{\prime \prime}(0)\right) .
$$

Repetindo o processo acima duas vezes, obtemos as fórmulas de $t_{2}^{(3)}(0)$ e $t_{2}^{(4)}(0)$ :

$$
t_{2}^{(3)}(0)=\frac{-u_{3}(2 \pi)-3 \dot{u}_{1}(2 \pi) t_{2}^{\prime \prime}(0)}{u_{0}(2 \pi)}
$$

e

$$
t_{2}^{(4)}(0)=\frac{-u_{4}(2 \pi)-4 \dot{u}_{2}(2 \pi) t_{2}^{\prime \prime}(0)-4 \dot{u}_{1}(2 \pi) t_{2}^{(3)}(0)}{\dot{u}_{0}(2 \pi)} .
$$

Utilizando as expressões de $u_{0}(t), u_{1}(t), u_{2}(t), t_{2}^{\prime \prime}(0)$ e $t_{2}^{(3)}(0)$,

$$
\begin{aligned}
t_{2}^{(3)}(0) & =\pi\left(\frac{1}{2} g^{\prime}(0) g^{\prime \prime}(0)-\frac{3}{2} g^{\prime}(0) f^{\prime \prime}(0)+\frac{17}{3} g^{\prime}(0) f^{\prime}(0)^{2}-\frac{8}{3} g^{\prime}(0)^{2} f^{\prime}(0)\right. \\
& \left.-\frac{1}{2} g^{\prime \prime}(0) f^{\prime}(0)+\frac{1}{3} g^{\prime}(0)^{3}\right) ;
\end{aligned}
$$

e

$$
\begin{aligned}
t_{2}^{(4)}(0) & =\frac{33}{64} g^{\prime \prime}(0)^{2}-\frac{7}{16} g^{\prime}(0)^{4}-\frac{1}{8} g^{(4)}(0)-\frac{1}{3} g^{\prime}(0) g^{\prime \prime \prime}(0)+\frac{57}{16} g^{\prime}(0)^{2} f^{\prime \prime}(0) \\
& -\frac{21}{32} g^{\prime \prime}(0) f^{\prime \prime}(0)+\frac{119}{4} g^{\prime}(0) f^{\prime}(0)^{3}-\frac{203}{16} g^{\prime}(0)^{2} f^{\prime}(0)^{2}+\frac{91}{24} g^{\prime}(0)^{3} f^{\prime}(0) \\
& -\frac{89}{24} g^{\prime \prime}(0) f^{\prime}(0)^{2}-\frac{23}{48} g^{\prime}(0)^{2} g^{\prime \prime}(0)+\frac{11}{6} g^{\prime}(0) f^{\prime \prime \prime}(0)+\frac{3}{2} g^{\prime \prime \prime}(0) f^{\prime}(0) \\
& -\frac{263}{16} g^{\prime}(0) f^{\prime \prime}(0) f^{\prime}(0)+\frac{3}{16} g^{\prime}(0) g^{\prime \prime}(0) f^{\prime}(0) .
\end{aligned}
$$

Seja

$$
q_{k}\left(t_{2}\left(x_{0}\right), x_{0}\right)=\frac{\partial^{k} y_{2}}{\partial x_{0}^{k}}\left(t_{2}\left(x_{0}\right) ; x_{0}\right)
$$

Para todo $k \in \mathbb{N}$, derivando $q_{k-1}\left(t_{2}\left(x_{0}\right) ; x_{0}\right)$ com respeito à $x_{0}$ obtemos $q_{k}\left(t_{1}\left(x_{0}\right) ; x_{0}\right)$.

Fazendo $q_{k}\left(t_{2}\left(x_{0}\right), x_{0}\right)=0$ e tomando $x_{0}=0$, encontramos uma fórmula para o cálculo de 
$t_{2}^{(k)}(0)$

Note que, para todo natural $k$, ao aplicarmos a Regra da Cadeia em (5.19), o termo $t_{2}^{(k)}(0)$ sempre será múltiplo de $\dot{u}_{0}(2 \pi)$ que é não nulo. Logo, $t_{2}^{(k)}(0)$ está bem definido.

Usando que $t_{2}^{\prime}(0)=0$ e $t_{2}(0)=2 \pi$, da Regra da Cadeia para funções de duas variáveis, segue que

$$
t_{2}^{(k)}(0)=-u_{k}(2 \pi)+G_{2}^{k}\left[t_{2}^{2}(0), \ldots, t_{2}^{(k-1)}(0), \dot{u}_{1}(2 \pi), \ldots, \dot{u}_{k-1}(2 \pi)\right] .
$$

\subsubsection{Fórmulas das Derivadas de $\tau, t_{1}, t_{2}$}

Para facilitar futuros cálculos, os resultados das derivadas de $\tau, t_{1}$ e $t_{2}$ em zero até a quarta ordem seguem no teorema.

Teorema 5.2.1 (Fórmula das Derivadas de $\tau, t_{1}, t_{2}$ ). Supondo que $f$ e $g$ são funções de ordem $C^{4}$ (no minimo), $f(0)=1$ e $g(0)=1$. Então,

$$
\begin{aligned}
& \tau^{\prime}(0)=0 ; \\
& \tau^{\prime \prime}(0)=\pi\left(\frac{5}{3} f^{\prime}(0)^{2}-\frac{3}{4} f^{\prime \prime}(0)\right) \\
& \tau^{(3)}(0)=\pi\left(-\frac{3}{2} f^{\prime \prime}(0) f^{\prime}(0)+\frac{10}{3} f^{\prime}(0)^{3}\right), \\
& \tau^{(4)}(0)=\pi\left(7 f^{\prime}(0) f^{\prime \prime \prime}(0)-\frac{275}{8} f^{\prime}(0)^{2} f^{\prime \prime}(0)+\frac{171}{64} f^{\prime \prime}(0)^{2}+\frac{385}{12} f^{\prime}(0)^{4}-\frac{5}{8} f^{(4)}(0)\right), \\
& t_{1}^{\prime}(0)=0, \\
& t_{1}^{\prime \prime}(0)=\frac{\pi}{12}\left(10 f^{\prime}(0) g^{\prime}(0)+10 g^{\prime}(0)^{2}-9 g^{\prime \prime}(0)\right) \text {, } \\
& t_{1}^{(3)}(0)=\pi\left(\frac{5}{6} g^{\prime}(0)^{2} f^{\prime}(0)-\frac{5}{6} g^{\prime}(0)^{3}+\frac{3}{4} g^{\prime}(0) g^{\prime \prime}(0)-\frac{3}{4} g^{\prime}(0) f^{\prime \prime}(0)-\frac{3}{2} g^{\prime \prime}(0) f^{\prime}(0)+\frac{10}{3} g^{\prime}(0) f^{\prime}(0)^{2}\right), \\
& t_{1}^{(4)}(0)=\pi\left(-\frac{5}{8} g^{(4)}(0)+\frac{249}{64} g^{\prime \prime}(0)^{2}-\frac{39}{32} g^{\prime \prime}(0) f^{\prime \prime}(0)-\frac{575}{48} g^{\prime}(0) g^{\prime \prime}(0) f^{\prime}(0)-\frac{157}{16} g^{\prime}(0) f^{\prime \prime}(0) f^{\prime}(0)\right. \\
& +\frac{259}{48} g^{\prime}(0)^{4}+\frac{7}{3} g^{\prime}(0) g^{\prime \prime \prime}(0)+\frac{7}{2} g^{\prime \prime \prime}(0) f^{\prime}(0)+\frac{7}{6} g^{\prime}(0) f^{\prime \prime \prime}(0)-\frac{91}{24} g^{\prime \prime}(0) f^{\prime}(0)^{2}+\frac{223}{12} g^{\prime}(0) f^{\prime}(0)^{3} \\
& \left.+\frac{43}{16} g^{\prime}(0)^{2} f^{\prime \prime}(0)+\frac{89}{8} g^{\prime}(0)^{3} f^{\prime}(0)-\frac{145}{48} g^{\prime}(0)^{2} f^{\prime}(0)^{2}-\frac{551}{48} g^{\prime}(0)^{2} g^{\prime \prime}(0)\right) \text {. } \\
& t_{2}^{\prime}(0)=0 \\
& t_{2}^{\prime \prime}(0)=\frac{\pi}{12}\left(14 f^{\prime}(0) g^{\prime}(0)-2 g^{\prime}(0)^{2}-3 g^{\prime \prime}(0)\right), \\
& t_{2}^{(3)}(0)=\pi\left(\frac{1}{2} g^{\prime}(0) g^{\prime \prime}(0)-\frac{3}{2} g^{\prime}(0) f^{\prime \prime}(0)+\frac{17}{3} g^{\prime}(0) f^{\prime}(0)^{2}-\frac{8}{3} g^{\prime}(0)^{2} f^{\prime}(0)-\frac{1}{2} g^{\prime \prime}(0) f^{\prime}(0)+\frac{1}{3} g^{\prime}(0)^{3}\right),
\end{aligned}
$$




$$
\begin{aligned}
t_{2}^{(4)}(0) & =\frac{33}{64} g^{\prime \prime}(0)^{2}-\frac{7}{16} g^{\prime}(0)^{4}-\frac{1}{8} g^{(4)}(0)-\frac{1}{3} g^{\prime}(0) g^{\prime \prime \prime}(0)+\frac{57}{16} g^{\prime}(0)^{2} f^{\prime \prime}(0) \\
& -\frac{21}{32} g^{\prime \prime}(0) f^{\prime \prime}(0)+\frac{119}{4} g^{\prime}(0) f^{\prime}(0)^{3}-\frac{203}{16} g^{\prime}(0)^{2} f^{\prime}(0)^{2}+\frac{91}{24} g^{\prime}(0)^{3} f^{\prime}(0) \\
& -\frac{89}{24} g^{\prime \prime}(0) f^{\prime}(0)^{2}-\frac{23}{48} g^{\prime}(0)^{2} g^{\prime \prime}(0)+\frac{11}{6} g^{\prime}(0) f^{\prime \prime \prime}(0)+\frac{3}{2} g^{\prime \prime \prime}(0) f^{\prime}(0) \\
& -\frac{263}{16} g^{\prime}(0) f^{\prime \prime}(0) f^{\prime}(0)+\frac{3}{16} g^{\prime}(0) g^{\prime \prime}(0) f^{\prime}(0) .
\end{aligned}
$$

\subsection{Propriedades das derivadas de $\tau, t_{1}, t_{2}$}

Esta seção é dedicada a enunciar e demonstrar alguns lemas que nos fornecem propriedades importantes das derivadas das funções $\tau, t_{1}$ e $t_{2}$ em zero.

Nos cálculos que seguem utilizaremos as seguintes fórmulas:

- Para $k=2 q$,

$$
\int \cos (t)^{k} d t=\frac{(2 q-1) ! !}{(2 q) ! !}\left[\sin (t) \sum_{p=0}^{q-1}\left(\frac{(2 p) ! !}{(2 p+1) ! !}\right) \cos (t)^{(2 p+1)}+t\right]
$$

- Para $k=2 q+1$

$$
\int \cos (t)^{k} d t=\frac{(2 q) ! !}{(2 q+1) ! !} \sin (t) \sum_{p=0}^{q} \frac{(2 p-1) ! !}{(2 p) ! !} \cos (t)^{(2 p)},
$$

em que o duplo fatorial ( $l$ )!! é definido da seguinte maneira: se $l$ é ímpar, é o produto dos naturais ímpares menores ou iguais a $l$. Se $l$ é par, é o produto dos naturais pares menores ou iguais a $l$.

Para demonstração das fórmulas veja [Wei].

Lema 5.3.1. Para todo $q \in \mathbb{N}, \tau^{(2 q)}(0)$ depende de $f^{(2 q)}(0)$ e $\tau^{(2 q+1)}(0)$ não depende de $f^{(2 q+1)}(0)$.

Demonstração. Na Subseção (5.2.1), obtivemos a expressão (5.13) para o cálculo de $\tau^{(k)}(0)$, ou seja, para todo natural $k$

$$
\tau^{(k)}(0)=\frac{1}{k+1} \dot{\mu}_{k+1}(2 \pi)+G^{k}\left[\tau^{(2)}(0), \ldots, \tau^{(2 k-1)}(0), \ddot{\mu}_{2}(2 \pi), \ldots, \ddot{\mu}_{k-1}(2 \pi)\right] .
$$

Como

$$
\mu_{k}(t)=F_{1}^{k}\left[\text { funções elementares, } f^{\prime}(0) \ldots, f^{(k-1)}(0)\right],
$$

$\mu_{k}(t)$ não depende $f^{(k)}(0)$ e o mesmo se mantém para as derivadas de $\mu_{k}(t)$ com respeito $t$. Portanto, na expressão de $\tau^{(k)}(0)$ o termo $f^{(k)}(0)$ só aparece em $\dot{\mu}_{k+1}(2 \pi)$.

A expressão de $\mu_{k+1}(t)$, para $k \neq 0$, é obtido resolvendo o sistema :

$$
\begin{gathered}
\ddot{\mu}_{k+1}(t)+\mu_{k+1}(t)=F^{k}\left[\mu_{1}(t), \ldots, \mu_{k}(t), f^{\prime}(0), f^{\prime \prime}(0), \ldots f^{(k)}(0)\right] \\
\mu_{k+1}(0)=0, \quad \dot{\mu}_{k+1}(0)=0 .
\end{gathered}
$$


O lado direito do sistema é obtido fazendo

$$
\mu_{k+1}\left(t ; x_{0}\right)=\frac{\partial^{k+1}}{\partial x_{0}^{k+1}}\left(-x\left(t ; x_{0}\right) f\left(x\left(t ; x_{0}\right)\right),\right.
$$

e tomando $x_{0}=0$.

Aplicando a Regra da Cadeia sucessivamente e fazendo $x_{0}=0$, observamos que, para todo natural $k$, o termo de $\ddot{\mu}_{k+1}\left(t ; x_{0}\right)$ que depende de $f^{(k)}(0)$ é da forma $-(k+1)\left(\mu_{1}(t)\right)^{k+1} f^{(k)}(0)$.

Usando que $\mu_{1}(t)=\cos (t)$ o termo é $(k+1)(\cos (t))^{k+1} f^{(k)}(0)$.

Note que na expressão de $\tau^{k}(0)$ o termo $\dot{\mu}_{k+1}(2 \pi)$ está multiplicado por $A_{k}=\frac{1}{k+1}$. Logo, o sistema pode ser reescrito como

$$
\begin{gathered}
\ddot{\mu}_{k+1}(t)+\mu_{k+1}(t)=-\cos (t)^{k+1} f^{(k)}(0)-\bar{F}\left[\mu_{1}(t), \ldots, \mu_{k}(t), f^{\prime}(0), f^{\prime \prime}(0), \ldots f^{(k-1)}(0)\right] \\
\mu_{k+1}(0)=0, \quad \dot{\mu}_{k+1}(0)=0 .
\end{gathered}
$$

Note que podemos resolver os seguintes sistemas separadamente e depois somar suas soluções para obter $\mu_{k+1}(t)$

$$
\begin{gathered}
\ddot{\mu}_{k+1}(t)+\mu_{k+1}(t)=-\left(\cos (t)^{k+1}\right) f^{(k)}(0) \\
\mu_{k+1}(0)=0, \quad \dot{\mu}_{k+1}(0)=0
\end{gathered}
$$

e

$$
\begin{gathered}
\ddot{\mu}_{k+1}(t)+\mu_{k+1}(t)=-\bar{F}_{1}\left[\mu_{1}(t), \ldots, \mu_{k}(t), f^{\prime}(0), f^{\prime \prime}(0), \ldots f^{(k-1)}(0)\right] \\
\mu_{k+1}(0)=0, \quad \dot{\mu}_{k+1}(0)=0 .
\end{gathered}
$$

Como $f^{(k)}(0)$ aparece somente nas equações do primeiro sistema, resolveremos apenas esse sistema.

Estudaremos separadamente os casos em que $k$ é par e $k$ é ímpar.

- Caso $k=2 q$, para algum $q \in \mathbb{N}$.

$$
\begin{gathered}
\ddot{\mu}_{2 q+1}(t)+\mu_{2 q+1}(t)=-(\cos (t))^{2 q+1} f^{(2 q)}(0) \\
\mu_{2 q+1}(0)=0, \quad \dot{\mu}_{2 q+1}(0)=0
\end{gathered}
$$

Utilizando o Método da Variação dos Parâmetros a solução do sistema é:

$$
\begin{aligned}
\mu_{2 q+1}(t) & =f^{(2 q)}(0)\left[\left(\int \sin (t)(\cos (t))^{2 q+1} d t\right) \cos (t)-\left(\int(\cos (t))^{2 q+2} d t\right) \sin (t)\right. \\
& \left.+c_{1} \cos (t)+c_{2} \sin (t)\right]
\end{aligned}
$$

$\operatorname{com} c_{1}=-\left.\left(\int \sin (t)(\cos (t))^{2 q+1}\right)\right|_{t=0}$ e $c_{2}=+\left(\int \cos (t)^{2 q+2} d t\right)_{t=0}$.

Como, $\forall q \in \mathbb{N}$

$$
\int \sin (t)(\cos (t))^{2 q+1} d t=-\frac{1}{2 q+2} \cos ^{(2 q+2)}(t)
$$

$\mathrm{e}$

$$
\int(\cos (t))^{2 q+2} d t=\frac{(2 q+1) ! !}{(2 q+2) ! !}\left[\sin (t) \sum_{p=0}^{q} \frac{(2 p) ! !}{(2 p+1) ! !}(\cos (t))^{2 p+1}+t\right]
$$


então

$$
\begin{aligned}
\mu_{2 q+1}(t) & =f^{(2 q)}(0)\left(-\frac{1}{2 q+2}(\cos (t))^{2 k+3}+\frac{1}{2 q+2} \cos (t)\right) \\
& -f^{(2 q)}(0) \frac{(2 q+1) ! !}{(2 q+2) ! !}\left[(\sin (t))^{2} \sum_{p=0}^{q} \frac{(2 p) ! !}{(2 p+1) ! !}(\cos (t))^{2 p+1}+t \sin (t)\right]
\end{aligned}
$$

Derivando $\mu_{2 q+1}(t)$ com respeito a $t$ e calculando em $t=2 \pi$, temos

$$
\dot{\mu}_{2 q+1}(2 \pi)=-2 \pi\left(\frac{(2 q+1) ! !}{(2 q+2) ! !}\right) f^{(2 q)}(0)
$$

Como, para todo q natural, $-2 \pi\left(\frac{(2 q+1) ! !}{(2 q+2) ! !}\right) \neq 0$, concluímos que $\tau^{(2 q)}(0)$ depende de $f^{(2 q)}(0)$.

- Caso $k=2 q+1$, para algum $q \in \mathbb{N}$.

$$
\begin{gathered}
\ddot{\mu}_{2 q+2}(t)+\mu_{2 q+2}(t)=-(\cos (t))^{2 q+2} f^{(2 q+1)}(0) \\
\mu_{2 q+2}(0)=0, \quad \dot{\mu}_{2 q+2}(0)=0 .
\end{gathered}
$$

Utilizando o Método da Variação dos Parâmetros a solução do sistema é:

$$
\begin{aligned}
\mu_{2 q+2}(t) & =f^{(2 q+1)}(0)\left[\left(\int \sin (t)(\cos (t))^{2 q+2} d t\right) \cos (t)-\left(\int(\cos (t))^{2 q+3} d t\right) \sin (t)\right. \\
& \left.+c_{1} \cos (t)+c_{2} \sin (t)\right]
\end{aligned}
$$

$\operatorname{com} c_{1}=-\left.\left(\int \sin (t)(\cos (t))^{2 q+2} d t\right)\right|_{t=0}$ e $c_{2}=+\left(\int(\cos (t))^{2 q+3} d t\right)_{t=0}$.

Como, $\forall q \in \mathbb{N}$

$$
\int \sin (t)(\cos (t))^{2 q+2} d t=-\frac{1}{2 q+3} \cos (t)^{(2 q+3)}
$$

e

$$
\int(\cos (t))^{2 q+3} d t=\frac{(2 q+2) ! !}{(2 q+3) ! !}\left[\sin (t) \sum_{p=0}^{q+1} \frac{(2 p-1) ! !}{(2 p) ! !}(\cos (t))^{2 p}\right]
$$

então

$$
\begin{aligned}
\mu_{2 q+2}(t) & =f^{(2 q+1)}(0)\left[\left(-\frac{1}{2 q+3}(\cos (t))^{2 k+4}+\frac{1}{2 q+3} \cos (t)\right)\right] \\
& -f^{(2 q+1)}(0) \frac{(2 q+2) ! !}{(2 q+3) ! !}\left[(\sin (t))^{2} \sum_{p=0}^{q+1} \frac{(2 p-1) ! !}{(2 p) ! !}(\cos (t))^{2 p}\right]
\end{aligned}
$$

Derivando com respeito a $t$ e calculando em $t=2 \pi$, temos

$$
\dot{\mu}_{2 q+2}(2 \pi)=-f^{(2 q+1)}(0) \times 0=0, \forall q \in \mathbb{N} .
$$

Concluímos que, $\tau^{(2 q+1)}(0)$ não depende de $f^{(2 q+1)}(0)$. 
Lema 5.3.2. Para todo $q \in \mathbb{N}, t_{1}^{(2 q)}(0)$ depende de $g^{(2 q)}(0)$ e $t_{1}^{(2 q+1)}(0)$ não depende de $g^{(2 q+1)}(0)$. Demonstração. Na Seção (5.2.1), obtivemos a expressão (5.18) para o cálculo de $t_{1}^{(k)}(0)$, ou seja, para todo natural $k$

$$
t_{1}^{(k)}(0)=\dot{v}_{k}(2 \pi)+G_{1}^{k}\left[t_{1}^{(2)}(0), \ldots, t_{1}^{(k-1)}(0), \ddot{v}_{1}(2 \pi), \ldots, \ddot{v}_{k-1}(2 \pi)\right] .
$$

Como

$$
v_{k}(t)=F_{1}^{(k)}\left[\text { funções elementares; } f^{\prime}(0), \ldots, f^{(k-1)}(0), g^{\prime}(0), \ldots, g^{(k)}(0)\right] \text {. }
$$

O mesmo se mantém para as derivadas de $v_{k}(t)$ em relação a $t$, logo em $t_{1}^{k}(0)$ o termo que depende $g^{(k)}(0)$ só aparece em $\dot{v}_{k}(2 \pi)$.

A expressão de $v_{k}(t)$, para $k \neq 0$, é obtida resolvendo o sistema:

$$
\begin{gathered}
\ddot{v}_{k}+v_{k}=F^{k}\left[\mu_{1}, \ldots, \mu_{k}, v_{0}, \ldots, v_{k-1}, g^{\prime}(0), \ldots, g^{(k)}(0)\right], \\
v_{k}(0)=0, \quad \dot{v}_{k}(0)=0
\end{gathered}
$$

em que o lado direito da equação é obtido fazendo

$$
\ddot{v}_{k}\left(t ; x_{0}\right)=\frac{\partial^{k}}{\partial x_{0}^{k}}\left(-y_{1}\left(t ; x_{0}\right) g\left(x\left(t ; x_{0}\right)\right)\right), \quad \forall k \in \mathbb{N},
$$

e tomando $x_{0}=0$.

Aplicando a Regra da Cadeia e fazendo $x_{0}=0$, observamos que o termo de $\ddot{v}_{k}(t ; 0)$ que depende $g^{(k)}(0)$ é da forma $-v_{0}(t)\left(\mu_{1}(t)\right)^{k} g^{(k)}(0)$. Usando que $\mu_{1}(t)=\cos (t)$ e $v_{0}(t)=\cos (t)$, o sistema pode ser reescrito como

$$
\begin{gathered}
\ddot{v}_{k}+v_{k}=-g^{(k)}(0) \cos (t)^{(k+1)}-\bar{F}\left[\mu_{1}(t), \ldots, \mu_{k-1}(t), v_{1}(t), \ldots, v_{k-2}, g^{\prime}(0), \ldots, g^{(k-1)}(0)\right], \\
v_{k}(0)=0, \quad \dot{v}_{k}(0)=0 .
\end{gathered}
$$

Utilizando o mesmo raciocínio da demonstração anterior, resolveremos apenas o sistema

$$
\begin{gathered}
\ddot{v}_{k}+v_{k}=-g^{(k)}(0) \cos (t)^{(k+1)} \\
v_{k}(0)=0, \quad \dot{v}_{k}(0)=0 .
\end{gathered}
$$

Note que este sistema coincide com o sistema que resolvemos na demonstração do Lema (5.3.2). Assim, concluímos os mesmos resultados trocando $\tau$ por $t_{1}$ e $g$ por $f$. Ou seja, se $k=2 q$ então $t_{1}^{(2 q)}(0)$ depende de $g^{(2 q)}(0)$, e se $k=2 q+1$, então $t_{1}^{(2 q+1)}(0)$ não depende de $g^{(2 q+1)}(0)$.

Lema 5.3.3. Para todo $q \in \mathbb{N}, t_{2}^{(2 q)}(0)$ depende de $g^{(2 q)}(0)$ e $t_{2}^{(2 q+1)}(0)$ não depende $g^{(2 q+1)}(0)$.

Demonstração. Na Seção 5.2.1, obtivemos a expressão (5.22) para o cálculo de $t_{2}^{k}(0)$, ou seja, para todo $k$ natural

$$
t_{2}^{(k)}(0)=-u_{k}(2 \pi)+G_{2}^{k}\left[t_{2}^{2}(0), \ldots, t_{2}^{(k-1)}(0), \dot{u}_{1}(2 \pi), \ldots, \dot{u}_{k-1}(2 \pi)\right] .
$$


A expressão de $u_{k}(t)$ é da forma

$$
u_{k}(t)=F_{1}^{k}\left[\text { funções elementares, } f^{\prime}(0), \ldots, f^{(k-1)}(0), g^{\prime}(0), \ldots, g^{k}(0)\right] \text {. }
$$

Logo, em $t_{2}^{k}(0)$ o termo $g^{(k)}(0)$ só aparece em $u_{k}(t)$, que para $k \neq 0$ é obtido resolvendo o sistema

$$
\begin{gathered}
\ddot{u}_{k}+u_{k}=F^{k}\left[\mu_{1}(t), \ldots, \mu_{k}(t), u_{1}(t), \ldots, u_{k-1}(t), g^{\prime}(0) \ldots g^{(k)}(0)\right], \\
u_{k}(0)=0, \quad \dot{u}_{k}(0)=0,
\end{gathered}
$$

onde o lado direito é obtido fazendo

$$
\ddot{u}_{k}\left(t ; x_{0}\right)=\frac{\partial^{k}}{\partial x_{0}^{k}}\left(-y_{2}\left(t ; x_{0}\right) g\left(x\left(t ; x_{0}\right)\right)\right)
$$

e tomando $x_{0}=0$.

Aplicando a Regra da Cadeia e fazendo $x_{0}=0, \forall k \in \mathbb{N}$, o termo de $\ddot{u}_{k}(t ; 0)$ que depende de $g^{(k)}(0)$ é $u_{0}(t)\left(\mu_{1}(t)\right)^{k} g^{(k)}(0)$.

Usando que $u_{0}(t)=\sin (t)$ e $\mu_{1}(t)=\cos (t)$ o sistema pode ser reescrito na forma:

$$
\begin{gathered}
\ddot{u}_{k}+u_{k}=-g^{(k)}(0) \sin (t)(\cos (t))^{k}-\bar{F}^{k}\left[\mu_{1}(t), \ldots, \mu_{k}(t), u_{1}(t), \ldots, u_{k-1}, g^{\prime}(0), \ldots, g^{(k-1)}(0)\right], \\
u_{k}(0)=0, \quad \dot{u}_{k}(0)=0 .
\end{gathered}
$$

Como estamos interessados somente na parte da solução do sistema que depende de $g^{(k)}(0)$, resolveremos apenas o sistema:

$$
\begin{gathered}
\ddot{u}_{k}+u_{k}=-g^{(k)}(0) \sin (t)(\cos (t))^{k} \\
u_{k}(0)=0, \quad \dot{u}_{k}(0)=0 .
\end{gathered}
$$

- Caso $k=2 q$, para algum $q \in \mathbb{N}$.

O sistema é:

$$
\begin{gathered}
\ddot{u}_{2 q}+u_{2 q}=-g^{(2 q)}(0) \sin (t)(\cos (t))^{2 q} \\
u_{2 q}(0)=0, \quad \dot{u}_{2 q}(0)=0 .
\end{gathered}
$$

Utilizando o Método da Variação dos Parâmetros a solução é

$$
\begin{aligned}
u_{2 q}(t) & =g^{(2 q)}(0)\left[\left(\int(\sin (t))^{2}(\cos (t))^{2 q} d t\right) \cos (t)-\left(\int \sin (t)(\cos (t))^{2 q+1} d t\right) \sin (t)\right. \\
& \left.+c_{1} \cos (t)+c_{2} \sin (t)\right],
\end{aligned}
$$


e

$$
\begin{aligned}
\int \sin (t)^{2}(\cos (t))^{2 q} d t & =\int \cos (t)^{2 q} d t-\int \cos (t)^{2 q+2} d t \\
& =\left(\frac{(2 q-1) ! !}{(2 q) ! !}-\frac{(2 q+1) ! !}{(2 q+2) ! !}\right)\left[\sin (t) \sum_{p=0}^{q-1} \frac{(2 p) ! !}{(2 p+1) ! !}(\cos (t))^{2 p+1}+t\right] \\
& -\frac{1}{2 q+2} \sin (t)(\cos (t))^{(2 q+1)}
\end{aligned}
$$

então

$$
\begin{aligned}
u_{2 q}(t) & =g^{(2 q)}(0)\left(\frac{(2 q-1) ! !}{(2 q) ! !}-\frac{(2 q+1) ! !}{(2 q+2) ! !}\right)\left[\sin (t) \cos (t) \sum_{p=0}^{q-1} \frac{(2 p) ! !}{(2 p+1) ! !}(\cos (t))^{2 p+1}\right] \\
& +g^{(2 q)}(0)\left(\frac{(2 q-1) ! !}{(2 q) ! !}-\frac{(2 q+1) ! !}{(2 q+2) ! !}\right)(t \cos (t))-\frac{1}{2 q+2} \sin (t)
\end{aligned}
$$

e

$$
u_{2 q}(2 \pi)=+2 \pi\left(\frac{(2 q-1) ! !}{(2 q) ! !}-\frac{(2 q+1) ! !}{(2 q+2) ! !}\right) g^{(2 q)}(0) \neq 0, \quad \forall q \in \mathbb{N} \text {. }
$$

Concluímos que, $\forall q \in \mathbb{N}, t_{2}^{(2 q)}(0)$ depende de $g^{(2 q)}(0)$.

- Caso $k=2 q+1$, para algum $q \in \mathbb{N}$.

O sistema é:

$$
\begin{aligned}
\ddot{u}_{2 q+1}(t)+u_{2 q+1}(t) & =-\sin (t)(\cos (t))^{2 q+1} g^{(2 q+1)}(0) \\
u_{2 q+1}(0) & =0, \quad \dot{u}_{2 q+1}(0)=0
\end{aligned}
$$

Utilizando o Método da Variação dos Parâmetros a solução do sistema é:

$$
\begin{aligned}
u_{2 q+1}(t) & =g^{(2 q+1)}(0)\left[\left(\int(\sin (t))^{2}(\cos (t))^{2 q+1} d t\right) \cos (t)\right. \\
& \left.-\left(\int \sin (t)(\cos (t))^{2 q+2} d t\right) \sin (t)+c_{1} \cos (t)+c_{2} \sin (t)\right]
\end{aligned}
$$

$\operatorname{com} c_{1}=-\left.\left(\int(\sin (t))^{2}(\cos (t))^{2 q+1} d t\right)\right|_{t=0}$ e $c_{2}=\left.\left(\int \sin (t)(\cos (t))^{2 q+2} d t\right)\right|_{t=0}$.

Como $\forall q \in \mathbb{N}$,

$$
\int \sin (t)(\cos (t))^{2 q+2} d t=-\frac{1}{2 q+3} \cos (t)^{(2 q+3)}
$$

e

$$
\begin{aligned}
\int(\sin (t))^{2}(\cos (t))^{2 q+1} d t & =\int(\cos (t))^{2 q+1} d t-\int(\cos (t))^{2 q+3} d t \\
& =\left(\frac{(2 q) ! !}{(2 q+1) ! !}-\frac{(2 q+2) ! !}{(2 q+3) ! !}\right)\left(\sin (t) \sum_{p=0}^{q+1} \frac{(2 p-1) ! !}{(2 p) ! !}(\cos (t))^{2 p}\right) \\
& -\frac{1}{2 q+3} \cos (t)^{2 q+2} \sin (t)
\end{aligned}
$$


então

$$
\begin{aligned}
u_{2 q+1}(t) & =g^{(2 q+1)}(0)\left(\frac{(2 q) ! !}{(2 q+1) ! !}-\frac{(2 q+2) ! !}{(2 q+3) ! !}\right)\left[\sin (t) \sum_{p=0}^{q} \frac{(2 p-1) ! !}{(2 p) ! !}(\cos (t))^{2 p}\right] \\
& -g^{(2 q+1)}(0) \frac{1}{2 q+3} \sin (t)
\end{aligned}
$$

e

$$
u_{2 q+1}(2 \pi)=0 \times g^{(2 q+1)}(0) .
$$

Logo, $\forall q \in \mathbb{N}, t_{2}^{(2 q+1)}(0)$ não depende de $g^{(2 q+1)}(0)$. 


\section{Capítulo 6}

\section{Resultado Principal}

Neste capítulo provaremos o nosso resultado principal:

Teorema Principal: Considere o sistema (5.1). Suponha $f(x)$ analítica real, definida e positiva numa vizinhança da origem $I$, com $f(0)=1$.

i) $S e 3 f^{\prime \prime}(0)>4 f^{\prime}(0)^{2}$, então existem no máximo duas funções $g(x)$ analíticas reais, definidas e positivas em $I$, com $g(0)=1$, tal que o sistema possui $\tau\left(x_{0}\right)$-isocronismo. Uma delas tem

$$
\begin{aligned}
& g^{\prime}(0)=f^{\prime}(0)-\frac{1}{4} \sqrt{-24 f^{\prime}(0)^{2}+18 f^{\prime \prime}(0)} \\
& g^{\prime \prime}(0)=\frac{9 f^{\prime \prime}(0)}{4}+\frac{5 f^{\prime}(0)}{6} \sqrt{-24 f^{\prime}(0)^{2}+18 f^{\prime \prime}(0)}-\frac{5 f^{\prime}(0)^{2}}{3}
\end{aligned}
$$

e a outra:

$$
\begin{aligned}
& g^{\prime}(0)=f^{\prime}(0)+\frac{1}{4} \sqrt{-24 f^{\prime}(0)^{2}+18 f^{\prime \prime}(0)} \\
& g^{\prime \prime}(0)=\frac{9 f^{\prime \prime}(0)}{4}+\frac{5 f^{\prime}(0)}{6} \sqrt{-24 f^{\prime}(0)^{2}+18 f^{\prime \prime}(0)}-\frac{5 f^{\prime}(0)^{2}}{3} .
\end{aligned}
$$

Além disso, se $g(x)$ é uma dessas funções e $k$ é um natural não nulo, $g^{(k+1)}(0)$ fica completamente determinada por $j^{(k)} g(0)$ e $j^{(k+1)} f(0)$.

ii) Se $3 f^{\prime \prime}(0)<4 f^{\prime}(0)^{2}$, então não existe $g(x)$ analítica real, positiva e definida em $I$, tal que o sistema possui $\tau\left(x_{0}\right)$-isocronismo.

iii) Se $3 f^{\prime \prime}(0)=4 f^{\prime}(0)^{2}$ e g é analítica real, positiva e definida em $I$, com $g(0)=1$, tal que o sistema possui $\tau\left(x_{0}\right)$-isocronismo, então para $k$ natural impar não nulo, $g^{(k+1)}(0)$ fica completamente determinado por $j^{(k)} g(0)$ e $j^{(k+1)} f(0)$.

Para faciltar a demonstração dividimos o teorema em três partes: Teoremas 6.1.1, 6.1.2 e 6.1.3, e a desmonstração do Teorema 6.1.2 em dois Lemas: 6.1.1 e 6.1.2, os quais desmonstramos nas Subseções 6.1.1 e 6.1.2, respectivamente.

Os cálculos da prova do Lema 6.1.2 são extensos e repetitivos. Aqui, apresentaremos as principais ideias envolvidas e algumas contas. Os cálculos completos podem ser verificados no Apêndice B. 


\subsection{Resultados}

Teorema 6.1.1. Considere o sistema (5.1). São dadas $f(x)$ e $g(x)$ analíticas reais, definidas e positivas numa vizinhança da origem, $I$, com $f(0)=g(0)=1$ e $3 f^{\prime \prime}(0) \geq 4 f^{\prime}(0)^{2}$. Se $g(x)$ é tal que o sistema (5.1) possui $\tau\left(x_{0}\right)$-isocronismo, então as seguintes condições são satisfeitas:

$$
\begin{gathered}
g^{\prime}(0)=f^{\prime}(0)-\frac{1}{4} \sqrt{-24 f^{\prime}(0)^{2}+18 f^{\prime \prime}(0)}, \\
g^{\prime \prime}(0)=\frac{9 f^{\prime \prime}(0)}{4}-\frac{5 f^{\prime}(0)}{6} \sqrt{-24 f^{\prime}(0)^{2}+18 f^{\prime \prime}(0)}-\frac{5 f^{\prime}(0)^{2}}{3}
\end{gathered}
$$

ou

$$
\begin{gathered}
g^{\prime}(0)=f^{\prime}(0)+\frac{1}{4} \sqrt{-24 f^{\prime}(0)^{2}+18 f^{\prime \prime}(0)}, \\
g^{\prime \prime}(0)=\frac{9 f^{\prime \prime}(0)}{4}+\frac{5 f^{\prime}(0)}{6} \sqrt{-24 f^{\prime}(0)^{2}+18 f^{\prime \prime}(0)}-\frac{5 f^{\prime}(0)^{2}}{3} .
\end{gathered}
$$

Demonstração. Aplicando o Teorema 5.1.2 para as derivadas pares de $\tau, t_{1}$ e $t_{2}$ calculadas em 0, temos

$$
\tau^{(2 q)}(0)=t_{1}^{(2 q)}(0)=t_{2}^{(2 q)}(0), \quad \forall q \in \mathbb{N} .
$$

Para $q=1$, fazendo $\tau^{(2)}(0)-t_{1}^{(2)}(0)=0, \tau^{(2)}(0)-t_{2}^{(2)}(0)=0$ e utilizando as fórmulas dadas no Teorema 5.2.1, obtemos, respectivamente, as seguintes expressões para o cálculo de $g^{\prime \prime}(0)$

$$
g^{\prime \prime}(0)=-\frac{20}{9} f^{\prime}(0)^{2}+f^{\prime \prime}(0)+\frac{10}{9} g^{\prime}(0) f^{\prime}(0)+\frac{10}{9} g^{\prime}(0)^{2} .
$$

$\mathrm{e}$

$$
g^{\prime \prime}(0)=-\frac{20}{3} f^{\prime}(0)^{2}+3 f^{\prime \prime}(0)+\frac{14}{3} f^{\prime}(0) g^{\prime}(0)-\frac{2}{3} g^{\prime}(0)^{2} .
$$

Como as duas expressões devem ser iguais:

$$
\frac{8}{9} g^{\prime}(0)^{2}-\frac{16}{9} g^{\prime}(0) f^{\prime}(0)+\frac{20}{9} f^{\prime}(0)^{2}-f^{\prime \prime}(0)=0
$$

Resolvendo a equação do segundo grau em $g^{\prime}(0)$, obtemos as duas possibilidades (6.1) e (6.3)

$$
g^{\prime}(0)=f^{\prime}(0)-\frac{1}{4} \sqrt{-24 f^{\prime}(0)^{2}+18 f^{\prime \prime}(0)}
$$

$\mathrm{Ou}$

$$
g^{\prime}(0)=f^{\prime}(0)+\frac{1}{4} \sqrt{-24 f^{\prime}(0)^{2}+18 f^{\prime \prime}(0)} .
$$

Para o cálculo de $g^{\prime \prime}(0)$ em cada um desses casos, obtemos, respectivamente, (6.3) e (6.4)

$$
g^{\prime \prime}(0)=\frac{9}{4} f^{\prime \prime}(0)-\frac{5}{6} f^{\prime}(0) \sqrt{-24 f^{\prime}(0)^{2}+18 f^{\prime \prime}(0)}-\frac{5}{3} f^{\prime}(0)^{2}
$$

$\mathrm{e}$

$$
g^{\prime \prime}(0)=\frac{9}{4} f^{\prime \prime}(0)+\frac{5}{6} f^{\prime}(0) \sqrt{-24 f^{\prime}(0)^{2}+18 f^{\prime \prime}(0)}-\frac{5}{3} f^{\prime}(0)^{2} .
$$


Corolário 6.1.1. Seja $f(x)$ analítica real, definida e positiva numa vizinhança da origem, I, com $f(0)=1$. Se $3 f^{\prime \prime}(0)<4 f^{\prime}(0)^{2}$, então não existe função $g(x)$ analítica real, definida e positiva em $I$, com $g(0)=1$ tal que o sistema (5.1) possua $\tau\left(x_{0}\right)$-isocronismo.

Demonstração. No Teorema 6.1.1, mostramos que se existe $g(x)$ analítica real tal que o sistema (5.1) possui $\tau\left(x_{0}\right)$-isocronismo, então

$$
g^{\prime}(0)=f^{\prime}(0) \pm \frac{1}{4} \sqrt{-24 f^{\prime}(0)^{2}+18 f^{\prime \prime}(0)}
$$

Se $f(0)<\frac{4 f^{\prime}(0)^{2}}{3}$, a expressão de $g^{\prime}(0)$ não está definida em $\mathbb{R}$. Portanto, não existe $g(x)$ real tal que (5.1) possua $\tau\left(x_{0}\right)$-isocronismo.

Teorema 6.1.2. Suponha que $f(x)$ é uma função analítica real e positiva em uma vizinhança da origem, $I$, com $f(0)=1$ e $3 f^{\prime \prime}(0)>4 f^{\prime}(0)^{2}$. Então existem no máximo duas funções analíticas reais $g(x)$ definidas em $I$, com $g(0)=1$, tais que o sistema (5.1) tenha $\tau\left(x_{0}\right)$-isocronismo. Além disso, se $g(x)$ for uma dessas funções e $k$ é um natural não nulo, $g^{(k+1)}(0)$ fica completamente determinado por $j^{(k)} g(0)$ e $j^{(k+1)} f(0)$.

Demonstração. Aplicando o Teorema 5.1.2 para as derivadas pares de $\tau, t_{1}$ e $t_{2}$ calculadas em 0 , temos

$$
\tau^{(2 q)}(0)=t_{1}^{(2 q)}(0)=t_{2}^{(2 q)}(0) \quad \forall q \in \mathbb{N} .
$$

Para $q>1$, dos Lemas (5.3.2) e (5.3.3) segue, respectivamente, que $t_{1}^{(2 q)}(0)$ e $t_{2}^{(2 q)}(0)$ possuem $g^{(2 q)}(0)$ em suas expressões.

Na demonstração desses lemas calculamos os coeficientes de $g^{(2 q)}(0)$ e mostramos que são não nulos. Assim, fazendo $\tau^{(2 q)}(0)-t_{1}^{(2 q)}(0)=0$ e isolando $g^{(2 q)}(0)$, obtemos:

$$
g^{(2 q)}(0)=\frac{H_{1}^{2 q}\left[f^{\prime}(0), \ldots, f^{(2 q)}(0), g^{\prime}(0), \ldots, g^{(2 q-1)}(0)\right]}{-2 \pi\left(\frac{(2 q+1) ! !}{(2 q+2) ! !}\right)} .
$$

Fazendo o mesmo para $\tau^{(2 q)}(0)-t_{2}^{(2 q)}(0)=0$, temos

$$
g^{(2 q)}(0)=\frac{H_{2}^{2 q}\left[f^{\prime}(0), \ldots, f^{(2 q)}(0), g^{\prime}(0), \ldots, g^{(2 q-1)}(0)\right]}{-2 \pi\left(\frac{(2 q-1) ! !}{(2 q) ! !}-\frac{(2 q+1) ! !}{(2 q+2) ! !}\right)}
$$

Note que o termo $f^{(2 q)}(0)$ está presente nas duas expressões (Lema 5.3.1).

Utilizaremos os lemas abaixo para conclusão da demonstração do resultado. As provas dos lemas serão feitas, respectivamente, nas Subseções 6.1.1 e 6.1.2.

Lema 6.1.1. Para todo natural $q>1$, igualando as expressões $(6.5)$ e (6.6), obtemos que $g^{(2 q-1)}(0)$ é dado pela expressão:

$$
\Delta(2 q-1) g^{(2 q-1)}(0)=\bar{H}^{2 q}\left[f^{\prime}(0), \ldots, f^{(2 q)}(0), g^{\prime}(0), \ldots, g^{(2 q-2)}(0)\right],
$$

em que $\Delta(2 q-1)=C^{2 q} g^{\prime}(0)+D^{2 q} f^{\prime}(0), \quad C^{2 q}, D^{2 q} \in \mathbb{R}$, não nulos.

Aqui, apesar da notação $C^{2 q}$ e $D^{2 q}$, estes coeficientes não dependem de $g^{(2 q)}(0)$. 
Lema 6.1.2. $\operatorname{Em}(6.7), C^{2 q}=-D^{2 q}$, para todo $q$ natural.

Dos lemas acima, concluímos que $\Delta(2 q-1)=0$ se, e somente se, $g^{\prime}(0)=f^{\prime}(0)$.

Dado o jato $k$ de $g(x)$ em 0 no caso em que (5.1) possui tal $\tau\left(x_{0}\right)$-isocronismo, segue do Teorema (6.1.1) que

$$
g^{\prime}(0)=f^{\prime}(0) \pm \frac{1}{4} \sqrt{-24 f^{\prime}(0)^{2}+18 f^{\prime \prime}(0)}
$$

ou seja,

$$
g^{\prime}(0)-f^{\prime}(0)= \pm \frac{1}{4} \sqrt{-24 f^{\prime}(0)^{2}+18 f^{\prime \prime}(0)} .
$$

Como $3 f^{\prime \prime}(0)>4 f^{\prime}(0)^{2}$, então $g^{\prime}(0)-f^{\prime}(0) \neq 0$. Assim, para todo $q$ natural, a partir de $j^{(2 q-2)} g(0)$ no caso em que (5.1) possui $\tau\left(x_{0}\right)$-isocronismo, a expressão (6.7) para o cálculo de $g^{(2 q-1)}$ está bem definida. $\mathrm{E} j^{(2 q-1)} g(0)$ fica unicamente determinado.

A partir de $j^{(2 q-1)} g(0)$, utilizando a expressão (6.5) ou a expressão (6.6) calcula-se $g^{(2 q)}(0)$ e $j^{(2 q)} g(0)$ fica unicamente determinado.

No Teorema 6.1.1 são obtidas duas condições distintas sobre as derivadas $g^{\prime}(0)$ e $g^{\prime \prime}(0)$. Portanto, temos dois jatos distintos de ordem $k=2$ para $g(x)$ em zero, um dado pelas condições (6.1) e (6.2) e o outro dado pelas condições (6.3) e (6.4). Ao calcularmos $g^{(3)}(0)$, obtemos dois valores, um obtido ao utilizarmos (6.1) e (6.2) e o outro obtido ao utilizarmos (6.3) e (6.4). Logo, temos duas possibilidades para jatos distintos de ordem $k=3$ de $g(x)$ em zero. O mesmo vale para as derivadas de $g(x)$ em zero de ordem maior que 3 .

Concluímos que existem no máximo duas funções $g(x)$ tais que o sistema (5.1) possui $\tau\left(x_{0}\right)$ isocronismo.

Teorema 6.1.3. Seja $f(x)$ analítica real e definida positiva em uma vizinhança da origem, $I$, com $f(0)=1$ e $3 f^{\prime \prime}(0)=4 f^{\prime}(0)^{2}$. Seja $j^{(k)} g(0)$, com $k$ impar, tal que $g(x)$ é analítica real e positiva $I$, com $g(0)=1$. Se $g(x)$ é tal que o sistema (5.1) possui $\tau\left(x_{0}\right)$-isocronismo, então $g^{(k+1)}(0)$ fica completamente determinado por $j^{(k)} g(0)$ e $j^{(k+1)} f(0)$.

Demonstração. Como $3 f^{\prime \prime}(0)=4 f^{\prime}(0)^{2}$ e $g(x)$ é tal que o sistema (5.1) possui $\tau\left(x_{0}\right)$-isocronismo, então $g^{\prime}(0)=f^{\prime}(0)$. Assim, para $q$ natural, não conseguimos determinar $j^{(2 q-1)}(0)$ usando $j^{(2 q-2)} g(0)$ em (6.7), pois $\Delta(2 q-1)$ é nulo.

Por outro lado, dado $j^{2 q-1} g(0)$, utilizando (6.5) ou (6.6) calcula-se $g^{(2 q)}(0)$. E $j^{2 q} g(0)$ fica unicamente determinado.

No que segue, faremos as demonstrações dos Lemas 6.1.1 e 6.1.2.

\subsubsection{Demonstração do Lema 6.1.1}

Demonstração. Vamos calcular os coeficientes de $g^{(2 q-1)}(0)$ nas expressões de (6.5) e (6.6).

Para cada $q$ natural, a expressão para o cálculo de $t_{1}^{(2 q)}(0)$ é

$$
t_{1}^{(2 q)}(0)=\dot{v}_{2 q}(2 \pi)+G_{1}^{2 q}\left[t_{1}^{\prime}(0), \ldots, t_{1}^{(2 q-1)}(0), \ddot{v}_{1}(2 \pi), \ldots, \ddot{v}_{2 q-1}(2 \pi)\right] .
$$

Como, para todo natural $l$,

$$
v_{l}(t)=F_{1}\left[\text { funções elementares, } f^{\prime}(0), \ldots, f^{(l-1)}(0), g^{\prime}(0) \ldots, g^{(l)}(0)\right]
$$


e $t_{1}^{(2 q-1)}(0)$ não possui $g^{2 q-1}(0)$ na sua expressão (Lema 5.3.2), então os termos de (6.8) que dependem de $g^{2 q-1}(0)$ provêm de $\dot{v}_{2 q}(2 \pi)$ ou $\ddot{v}_{2 q-1}(2 \pi)$.

A expressão (6.8) foi obtida derivando a igualdade

$$
\dot{y}_{1}\left(t_{1}\left(x_{0}\right), x_{0}\right)=0, \quad t_{1}(2 \pi)=0
$$

repetidamente com respeito a $x_{0}$ e fazendo $x_{0}=0$.

Para todo natural $q$, ao aplicarmos a regra da cadeia na igualdade acima, $\ddot{v}_{2 q-1}(2 \pi)$ sempre será multiplo de $t_{1}^{\prime}(0)$ que é nulo. Portanto, $g^{2 q-1}(0)$ na expressão 6.8 provêm somente de $\dot{v}_{2 q}(2 \pi)$.

A expressão de $v_{2 q}(t)$ é obtida resolvendo o sistema

$$
\begin{gathered}
\ddot{v}_{2 q}(t)+v_{2 q}(t)=F^{2 q}\left[\mu_{1}(t), \ldots \mu_{2 q}(t), v_{0}(t), \ldots, v_{2 q-1}(t), g^{\prime}(0), \ldots, g^{(2 q)}(0)\right], \\
v_{2 q}(0)=0, \quad \dot{v}_{2 q}(0)=0
\end{gathered}
$$

que é obtido fazendo

$$
\ddot{v}_{2 q}\left(t ; x_{0}\right)=\frac{\partial^{2 q}}{\partial x_{0}^{2 q}}\left(-y_{1}\left(t ; x_{0}\right) g\left(x\left(t ; x_{0}\right)\right)\right)
$$

e tomando $x_{0}=0$.

Aplicando o Teorema de Schwarz sucessivamente, observamos que os termos do lado direito do sistema que dependem $g^{(2 q-1)}(0)$ são da forma:

$$
\begin{gathered}
2 q \frac{\partial y_{1}}{\partial x_{0}}\left(t ; x_{0}\right)\left(\frac{\partial x}{\partial x_{0}}\left(t ; x_{0}\right)\right)^{2 q-1} g^{(2 q-1)}(0), \quad a_{2 q} y_{1}\left(t ; x_{0}\right) \frac{\partial^{2} x}{\partial x_{0}^{2}}\left(t ; x_{0}\right)\left(\frac{\partial x}{\partial x_{0}}\left(t ; x_{0}\right)\right)^{2 q-2} g^{(2 q-1)}(0), \\
2 q \frac{\partial x}{\partial x_{0}}\left(t ; x_{0}\right)\left(\frac{\partial^{2 q-1} y_{1}}{\partial x_{0}^{2 q-1}}\left(t ; x_{0}\right)\right) g^{\prime}(0),
\end{gathered}
$$

em que $a_{2 q}=q(2 q-1)$.

Tomando $x_{0}=0$, os termos ficam:

$$
2 q v_{1}(t) \mu_{1}(t)^{2 q-1} g^{(2 q-1)}(0), \quad a_{2 q} v_{0}(t) \mu_{1}(t)^{2 q-2}(t) \mu_{2}(t) g^{(2 q-1)(0)}, \quad 2 q \mu_{1}(t) v_{2 q-1}(t) g^{\prime}(0) .
$$

Só estamos interessados na parte de $v_{2 q-1}(t)$ que depende $g^{(2 q-1)}(0)$, ou seja, a parte da dada pela resolução do sistema:

$$
\begin{gathered}
\ddot{\bar{v}}_{2 q-1}+\bar{v}_{2 q-1}=-v_{0}(t) \mu_{1}(t)^{2 q-1}(t) g^{(2 q-1)}(0) \\
\bar{v}_{2 q-1}(0)=0, \quad \dot{\bar{v}}_{2 q-1}(0)=0 .
\end{gathered}
$$

Logo, os termos que dependem de $g^{(2 q-1)}(0)$ a expressão de $t_{1}^{(2 q)}(0)$ são obtidos resolvendo o sistema

$$
\begin{aligned}
\ddot{v}_{2 q}+v_{2 q} & =-2 q \mu_{1}(t) v_{1}(t)^{2 q-1} g^{(2 q-1)}(0) \\
& -a_{2 q} v_{0}(t) \mu_{1}(t)^{2 q-2}(t) \mu_{2}(t) g^{(2 q-1)}(0) \\
- & 2 q \mu_{1}(t) \bar{v}_{2 q-1}(t) g^{\prime}(0) . \\
& v_{2 q}(0)=0, \quad \dot{v}_{2 q}(0)=0 .
\end{aligned}
$$

Note que $v_{1}(t), \mu_{2}(t)$ e $\bar{v}_{2 q-1}(t)$ dependem, respectivamente, somente de $g^{\prime}(0), f^{\prime}(0)$ e $g^{(2 q-1)}(0)$. 
Assim, a solução desse sistema será da forma:

$$
v_{2 q}(t)=I_{1}^{2 q}\left[\text { funções elementares; } g^{\prime}(0) g^{2 q-1}(0) ; f^{\prime}(0) g^{(2 q-1)}(0)\right] .
$$

Derivando $v_{2 q}(t)$ com respeito a $t$ e calculando em $t=2 \pi$ :

$$
\dot{v}_{2 q}(2 \pi)=\bar{I}_{1}^{2 q}\left[g^{\prime}(0) g^{2 q-1}(0) ; f^{\prime}(0) g^{(2 q-1)}(0)\right] .
$$

Analogamente, para todo natural $q$, os termos que dependem $g^{(2 q-1)}(0)$ na expressão de $t_{2}^{(2 q)}(0)$ são obtidos resolvendo o sistema:

$$
\begin{aligned}
\ddot{u}_{2 q}+u_{2 q} & =-2 q \mu_{1}(t) u_{1}(t)^{2 q-1} g^{(2 q-1)}(0) \\
& -a_{2 q} u_{0}(t) \mu_{1}(t)^{2 q-2}(t) \mu_{2}(t) g^{(2 q-1)(0)} \\
& -2 q \mu_{1}(t) \bar{u}_{2 q-1}(t) g^{\prime}(0) \\
& u_{2 q}(0)=0, \quad \dot{u}_{2 q}(0)=0
\end{aligned}
$$

em que $\bar{u}_{2 q-1}(t)$ é a solução do sistema

$$
\begin{gathered}
\ddot{\bar{u}}_{2 q-1}+\bar{u}_{2 q-1}=-u_{0}(t) \mu_{1}(t)^{2 q-1}(t) g^{(2 q-1)(0)} \\
\bar{u}_{2 q-1}(0)=0, \quad \dot{\bar{u}}_{2 q-1}(0)=0 .
\end{gathered}
$$

Note que $u_{1}(t), \mu_{2}(t)$ e $\bar{u}_{2 q-1}(t)$ dependem, respectivamente, somente de $g^{\prime}(0), f^{\prime}(0)$ e $g^{(2 q-1)}(0)$. Assim, a solução de (6.9) é da forma:

$$
u_{2 q}(t)=I_{2}^{2 q}\left[\text { funções elementares; } g^{\prime}(0) g^{2 q-1}(0) ; f^{\prime}(0) g^{(2 q-1)}(0)\right] \text {. }
$$

Calculando em $t=2 \pi$

$$
u_{2 q}(2 \pi)=\bar{I}_{2}^{2 q}\left[g^{\prime}(0) g^{(2 q-1)}(0) ; f^{\prime}(0) g^{(2 q-1)}(0)\right] .
$$

Portanto,

$$
g^{(2 q)}(0)=\frac{{\overline{H_{1}}}^{2 q}\left[f^{\prime}(0), \ldots, f^{(2 q)}(0) ; g^{\prime}(0), \ldots, g^{(2 q-2)}(0)\right]+\bar{I}_{1}^{2 q}\left[f^{\prime}(0) g^{(2 q-1)}(0), g^{\prime}(0) g^{(2 q-1)}(0)\right]}{-2 \pi\left(\frac{(2 q+1) ! !}{(2 q+2) ! !}\right)}
$$

e

$$
g^{(2 q)}(0)=\frac{{\overline{H_{2}}}^{2 q}\left[f^{\prime}(0), \ldots, f^{(2 q)}(0), g^{\prime}(0), \ldots, g^{(2 q-2)}(0)\right]+\bar{I}_{2}^{2 q}\left[f^{\prime}(0) g^{(2 q-1)}(0), g^{\prime}(0) g^{(2 q-1)}(0)\right]}{-2 \pi\left(\frac{(2 q-1) ! !}{(2 q) ! !}-\frac{(2 q+1) ! !}{(2 q+2)}\right)}
$$

Igualando as duas expressões, temos

$$
\frac{I_{1}^{2 q}\left[f^{\prime}(0) g^{(2 q-1)}(0), g^{\prime}(0) g^{(2 q-1)}(0)\right]}{2 \pi\left(\frac{(2 q+1) ! !}{(2 q+2) ! !}\right)}+\frac{I_{2}^{2 q}\left[f^{\prime}(0) g^{(2 q-1)}(0), g^{\prime}(0) g^{(2 q-1)}(0)\right]}{2 \pi\left(\frac{(2 q-1) ! !}{(2 q) ! !}-\frac{(2 q+1) ! !}{(2 q+2) ! !}\right)}
$$




$$
=\frac{{\overline{H_{1}}}^{2 q}\left[f^{\prime}(0), \ldots, f^{(2 q)}(0), g^{\prime}(0), \ldots, g^{(2 q-2)}(0)\right]}{-2 \pi\left(\frac{(2 q+1) ! !}{(2 q+2) ! !}\right)}+\frac{{\overline{H_{2}}}^{2 q}\left[f^{\prime}(0), \ldots, f^{(q)}(0), g^{\prime}(0), \ldots, g^{(2 q-2)}(0)\right]}{-2 \pi\left(\frac{(2 q-1) ! !}{(2 q) ! !}-\frac{(2 q+1) ! !}{(2 q+2) ! !}\right)}
$$

que pode ser reescrito como:

$$
\Delta(2 q-1) g^{(2 q-1)}(0)=\bar{H}^{2 q}\left[f^{\prime}(0), \ldots, f^{(2 q)}(0), g^{\prime}(0), \ldots, g^{2 q-2}(0)\right]
$$

em que

$$
\begin{aligned}
\Delta(2 q-1) & =\left(\frac{A_{1}^{2 q}}{2 \pi\left(\frac{(2 q+1) ! !}{(2 q+2) ! !}\right)}+\frac{B_{1}^{2 q}}{2 \pi\left(\frac{(2 q-1) ! !}{(2 q) ! !}-\frac{(2 q+1) ! !}{(2 q+2) ! !}\right)}\right) f^{\prime}(0) \\
& +\left(\frac{A_{2}^{2 q}}{2 \pi\left(\frac{(2 q+1) ! !}{(2 q+2) ! !}\right)}+\frac{B_{2}^{2 q}}{2 \pi\left(\frac{(2 q-1) ! !}{(2 q) ! !}-\frac{(2 q+1) ! !}{(2 q+2) ! !}\right)}\right) g^{\prime}(0),
\end{aligned}
$$

$\operatorname{com} A_{1}^{2 q}, A_{2}^{2 q}, B_{1}^{2 q}, B_{2}^{2 q} \in \mathbb{R}$

Seja

$$
C^{2 q}=\frac{A_{1}^{2 q}}{2 \pi\left(\frac{(2 q+1) ! !}{(2 q+2) ! !}\right)}+\frac{B_{1}^{2 q}}{2 \pi\left(\frac{(2 q-1) ! !}{(2 q) ! !}-\frac{(2 q+1) ! !}{(2 q+2) ! !}\right)}
$$

$\mathrm{e}$

$$
D^{2 q}=\frac{A_{2}^{2 q}}{2 \pi\left(\frac{(2 q+1) ! !}{(2 q+2) ! !}\right)}+\frac{B_{2}^{2 q}}{2 \pi\left(\frac{(2 q-1) ! !}{(2 q) ! !}-\frac{(2 q+1) ! !}{(2 q+2) ! !}\right)}
$$

Assim, obtemos a expressão (6.7).

\subsubsection{Demonstração do Lema 6.1.2}

Devemos calcular $A_{1}^{2 q}, B_{1}^{2 q}, A_{2}^{2 q}$ e $B_{2}^{2 q}$ e mostrar que $C^{2 q}=-D^{2 q}$, para todo $q$ natural. Para isso, iremos enunciar e provar alguns lemas auxiliares. Nas demonstrações não iremos explicitar todas as contas, os detalhes das contas encontram-se no Apêndice B.

Lema 6.1.3. Para todo q natural, vale

$$
A_{1}^{2 q}=-\frac{4}{3} a_{2 q} \pi(2 q+3)\left(\frac{(2 q-1) ! !}{(2 q+2) ! !}\right)
$$

Demonstração. Devemos resolver o sistema:

$$
\begin{gathered}
\ddot{v}_{2 q}+v_{2 q}=-a_{2 q} v_{0}(t) \mu_{1}(t)^{2 q-2}(t) \mu_{2}(t) g^{(2 q-1)}(0) \\
v_{2 q}(0)=0, \quad \dot{v}_{2 q}(0)=0
\end{gathered}
$$

derivar a solução obtida com respeito a $t$ e calcular em $t=2 \pi$. 
Substituindo $v_{0}(t), \mu_{1}(t)$ e $\mu_{2}(t)$, temos

$$
\begin{gathered}
\ddot{v}_{2 q}+v_{2 q}=-a_{2 q} \cos (t)^{2 q-1}\left(\frac{2}{3} \cos (t)+\frac{1}{3} \cos (2 t)-1\right) \\
v_{2 q}(0)=0, \quad \dot{v}_{2 q}(0)=0
\end{gathered}
$$

Vamos reescrever o sistema (6.14) em sistemas independentes e depois encontrar as soluções destes sistemas.

- Resolução do sistema $(S 1)$

$$
\begin{gathered}
\ddot{v}_{2 q}^{(1)}+v_{2 q}^{(1)}=-\frac{2}{3} a_{2 q} \cos (t)^{2 q} \\
v_{2 q}^{(1)}(0)=0, \quad \dot{v}_{2 q}^{(1)}(0)=0
\end{gathered}
$$

Seja $K_{1}=\frac{2}{3} a_{2 q}$.

Aplicando o Método da Variação dos Parâmetros a solução do sistema é

$$
\begin{aligned}
v_{2 q}^{(1)}(t) & =K_{1}\left(-\frac{1}{2 q+1}(\cos (t))^{2 q+2}+\frac{1}{2 q+1} \cos (t)\right) \\
& -K_{1} \frac{(2 q) ! !}{(2 q+1) ! !}\left((\sin (t))^{2} \sum_{p=0}^{q} \frac{(2 p-1) ! !}{(2 p) ! !}(\cos (t))^{2 p}\right) .
\end{aligned}
$$

- Resolução do sistema $(S 2)$

$$
\begin{gathered}
\ddot{v}_{2 q}^{(2)}+v_{2 q}^{(2)}=-\left(-a_{2 q} \cos (t)^{2 q-1}\right) \\
v_{2 q}^{(2)}(0)=0, \quad \dot{v}_{2 q}^{(2)}(0)=0
\end{gathered}
$$

Seja $k_{2}=-a_{2 q}$.

Aplicando o Método da Variação dos Parâmetros a solução do sistema é

$$
\begin{aligned}
v_{2 q}^{(2)}(t) & =K_{2}\left(-\frac{1}{2 q}(\cos (t))^{2 q+1}+\frac{1}{2 q} \cos (t)\right) \\
& -K_{2} \frac{(2 q-1) ! !}{(2 q) ! !}\left(\sin (t)^{2} \sum_{p=0}^{q-1} \frac{(2 p) ! !}{(2 p+1) ! !}(\cos (t))^{2 p+1}+t \sin (t)\right)
\end{aligned}
$$

- Resolução do sistema (S3)

$$
\begin{gathered}
\ddot{v}_{2 q}^{(3)}+v_{2 q}^{(3)}=-\frac{1}{3} a_{2 q} \cos (t)^{2 q-1} \cos (2 t) \\
v_{2 q}^{(3)}(0)=0, \quad \dot{v}_{2 q}^{(3)}(0)=0,
\end{gathered}
$$

Para todo $q$ natural,

$$
\cos (t)^{2 q-1} \cos (2 t)=2 \cos (t)^{2 q+1}-\cos (t)^{2 q-1} .
$$


Assim, $(S 3)$ pode ser reescrito como

$$
\begin{gathered}
\ddot{v}_{2 q}^{(3)}+v_{2 q}^{(3)}=-\frac{1}{3} a_{2 q}\left(2 \cos (t)^{2 q+1}-\cos (t)^{2 q-1}\right) \\
v_{2 q}^{(3)}(0)=0, \quad \dot{v}_{2 q}^{(3)}(0)=0
\end{gathered}
$$

- Resolução do sistema $(S 3)_{1}$

$$
\begin{gathered}
\ddot{v}_{2 q}^{(3)_{1}}+v_{2 q}^{(3)_{1}}=-\frac{2}{3} a_{2 q} \cos (t)^{2 q+1} \\
v_{2 q}^{(3)_{1}}(0)=0, \quad \dot{v}_{2 q}^{(3)_{1}}(0)=0
\end{gathered}
$$

Seja $\left(K_{3}\right)_{1}=\frac{2}{3} a_{2 q}$.

Aplicando o Método da Variação dos Parâmetros a solução do sistema é

$$
\begin{aligned}
v_{2 q}^{(3)_{1}}(t) & =\left(K_{3}\right)_{1}\left(-\frac{1}{2 q+2}(\cos (t))^{2 q+3}+\frac{1}{2 q+2} \cos (t)\right) \\
& -\left(K_{3}\right)_{1} \frac{(2 q+1) ! !}{(2 q+2) ! !}\left(\sin (t)^{2} \sum_{p=0}^{q} \frac{(2 p-1) ! !}{(2 p) ! !}(\cos (t))^{2 p}+t \sin (t)\right)
\end{aligned}
$$

- Resolução do sistema $(S 3)_{2}$

$$
\begin{gathered}
\ddot{v}_{2 q}^{(3)_{2}}+v_{2 q}^{(3)_{2}}=-\left(-\frac{1}{3} a_{2 q}\right) \cos (t)^{2 q-1} \\
v_{2 q}^{(3)_{2}}(0)=0, \quad \dot{v}_{2 q}^{(3)_{2}}(0)=0
\end{gathered}
$$

$\operatorname{Seja}\left(K_{3}\right)_{2}=-\frac{1}{3} a_{2 q}$

Aplicando o Método da Variação dos Parâmetros a solução do sistema é

$$
\begin{aligned}
v_{2 q}^{(3)_{2}}(t) & =\left(K_{3}\right)_{2}\left(-\frac{1}{2 q}(\cos (t))^{2 q+1}+\frac{1}{2 q} \cos (t)\right) \\
& -\left(K_{3}\right)_{2} \frac{(2 q-1) ! !}{(2 q) ! !}\left(\sin (t)^{2} \sum_{p=0}^{q-1} \frac{(2 p-1) ! !}{(2 p) ! !}(\cos (t))^{2 p}+t \sin (t)\right)
\end{aligned}
$$

A solução do sistema (6.14), $v_{2 q}(t)$, é a soma das soluções dos sistemas $S 1, S 2,(S 3)_{1}$ e $(S 3)_{2}$. Derivando $v_{2 q}(t)$ com respeito a $t$ e calculando em $t=2 \pi$, temos

$$
A_{1}^{2 q}=-2 \pi K_{2} \frac{(2 q-1) ! !}{(2 q) ! !}-2 \pi\left(K_{3}\right)_{1} \frac{(2 q+1) ! !}{(2 q+2) ! !}-2 \pi\left(K_{3}\right)_{2} \frac{(2 q-1) ! !}{(2 q) ! !}
$$

Substituindo os valores de $K_{2},\left(K_{3}\right)_{1}$ e $\left(K_{3}\right)_{2}$,

$$
A_{1}^{2 q}=-\frac{4 \pi}{3} a_{2 q} \frac{(2 q+1) ! !}{(2 q+2) ! !}+\frac{8 \pi}{3} a_{2 q} \frac{(2 q-1) ! !}{(2 q) ! !}
$$


Simplificando,

$$
A_{1}^{(2 q)}=\frac{4 \pi a_{2 q}}{3}(2 q+3)\left(\frac{(2 q-1) ! !}{(2 q+2) ! !}\right) .
$$

Lema 6.1.4. Para todo natural $q$, vale

$$
B_{1}^{2 q}=-\frac{4 \pi a_{2 q}}{3}(2 q+5)\left(\frac{(2 q-3) ! !}{(2 q+2) ! !}\right) .
$$

Demonstração. Devemos encontrar a solução do sistema:

$$
\begin{gathered}
\ddot{u}_{2 q}+u_{2 q}=-a_{2 q} u_{0}(t) \mu_{1}(t)^{2 q-2}(t) \mu_{2}(t) \\
u_{2 q}(0)=0, \quad \dot{u}_{2 q}(0)=0
\end{gathered}
$$

e calcular em $t=2 \pi$.

Substituindo $u_{0}(t), \mu_{1}(t)$ e $\mu_{2}(t)$, temos

$$
\begin{gathered}
\ddot{u}_{2 q}+u_{2 q}=-a_{2 q} \sin (t) \cos (t)^{2 q-2}\left(\frac{2}{3} \cos (t)+\frac{1}{3} \cos (2 t)-1\right) \\
u_{2 q}(0)=0, \quad \dot{u}_{2 q}(0)=0,
\end{gathered}
$$

Procedendo de maneira análoga a demonstração do lema anterior, obtemos $u_{2 q}(t)$.

Calculando em $t=2 \pi$, temos

$$
B_{1}^{2 q}=-\frac{8 \pi}{3} a_{2 q} \frac{(2 q-3) ! !}{(2 q-2) ! !}+4 \pi a_{2 q} \frac{(2 q-1) ! !}{(2 q) ! !}-\frac{4 \pi}{3} a_{2 q} \frac{(2 q+1) ! !}{(2 q+2) ! !} .
$$

Simplificando,

$$
B_{1}^{2 q}=-\frac{4 \pi a_{2 q}}{3}(2 q+5)\left(\frac{(2 q-3) ! !}{(2 q+2) ! !}\right)
$$

Lema 6.1.5. Para todo q natural, vale

$$
A_{2}^{2 q}=\frac{8 q(2 q+3)}{3}\left(\frac{(2 q-1) ! !}{(2 q+2) ! !}\right) .
$$

Demonstração. Devemos resolver o sistema:

$$
\begin{gathered}
\ddot{v}_{2 q}+v_{2 q}=-2 q \mu_{1}(t) v_{1}(t)^{2 q-1} g^{(2 q-1)}(0) \\
-2 q \mu_{1}(t) \bar{v}_{2 q-1}(t) g^{\prime}(0) \\
v_{2 q}(0)=0, \quad \dot{v}_{2 q}(0)=0
\end{gathered}
$$

em que $\bar{v}_{2 q-1}(t)$ é a solução do sistema:

$$
\begin{gathered}
\ddot{\bar{v}}_{2 q-1}+\bar{v}_{2 q-1}=-\cos (t)^{2 q} g^{(2 q-1)}(0) \\
\bar{v}_{2 q-1}(0)=0, \quad \dot{\bar{v}}_{2 q-1}(0)=0 .
\end{gathered}
$$


Substituindo os valores de $\mu_{1}(t), v_{1}(t)$ e $\bar{v}_{2 q-1}(t)$ em (6.19), temos

$$
\begin{gathered}
\ddot{\bar{v}}_{2 q-1}+\bar{v}_{2 q-1}=-\cos (t)^{2 q} g^{(2 q-1)}(0) \\
\bar{v}_{2 q-1}(0)=0, \quad \dot{\bar{v}}_{2 q-1}(0)=0
\end{gathered}
$$

cuja solução é

$$
\begin{aligned}
\bar{v}_{2 q-1}(t) & =g^{(2 q-1)}(0)\left[-\frac{1}{2 q+1} \cos (t)^{2 q+2}+\frac{1}{(2 q+1)} \cos (t)\right. \\
& \left.-\frac{(2 q) ! !}{(2 q+1) ! !}\left(\sin (t)^{2} \sum_{p=0}^{q} \frac{(2 p-1) ! !}{(2 p) ! !}(\cos (t))^{2 p}\right)\right] .
\end{aligned}
$$

Substituindo os valores de $\mu_{1}(t), v_{1}(t)$ e $\bar{v}_{2 q-1}(t)$ em (6.18), temos

$$
\begin{aligned}
\ddot{v}_{2 q}+v_{2 q} & =-2 q \cos (t)^{2 q-1}\left(\frac{1}{3} \cos (t)^{2}-\frac{2}{3}+\frac{1}{3} \cos (t)\right) g^{\prime}(0) g^{(2 q-1)}(0) \\
& -2 q \cos (t)\left(-\frac{1}{2 q+1} \cos (t)^{2 q+2}+\frac{1}{(2 q+1)} \cos (t)\right) g^{\prime}(0) g^{(2 q-1)}(0) \\
& -2 q \cos (t)\left[\frac{(2 q) ! !}{(2 q+1) ! !}\left(\sin (t)^{2} \sum_{p=0}^{q} \frac{(2 p-1) ! !}{(2 p) ! !}(\cos (t))^{2 p}\right)\right] g^{\prime}(0) g^{(2 q-1)}(0) .
\end{aligned}
$$

Utilizando o Método da Variação dos Paramêtros calculamos $v_{2 q}(t)$. Derivando com respeito $t$ e fazendo $t=2 \pi$,

$$
\begin{aligned}
A_{2}^{2 q} & =-\frac{4 q \pi}{3}\left(\frac{(2 q+1) ! !}{(2 q+2) ! !}\right)+\frac{8 q \pi}{3}\left(\frac{(2 q-1) ! !}{(2 q) ! !}\right)+\frac{4 q \pi}{2 q+1}\left(\frac{(2 q+3) ! !}{(2 q+4) ! !}\right) \\
& -4 q \pi\left(\frac{(2 q) ! !}{(2 q+1) ! !}\right) \sum_{p=0}^{q} \frac{(2 p-1) ! !}{(2 p) ! !}\left(\frac{(2 p+3) ! !}{(2 p+4) ! !}-\frac{(2 p+1) ! !}{(2 p+2) ! !}\right)
\end{aligned}
$$

Simplificando,

$$
A_{2}^{2 q}=\frac{4 q \pi}{3}(2 q+6)\left(\frac{(2 q-1) ! !}{(2 q+2) ! !}\right)+4 \pi q\left(\frac{(2 q) ! !}{(2 q+1) ! !}\right) \sum_{p=0}^{q-1} \frac{(2 p-1) ! !}{(2 p) ! !}\left(\frac{(2 p+1) ! !}{(2 p+4) ! !}\right) .
$$

Para completar a prova, utilizaremos a seguinte afirmação:

Afirmação 6.1.1. Para todo natural $q \geq 2$,

$$
\frac{2 q}{3}\left(\frac{(2 q-1) ! !}{(2 q+2) ! !}\right)=\frac{(2 q) ! !}{(2 q+1) ! !} \sum_{p=0}^{q-1} \frac{(2 p-1) ! !}{(2 p) ! !}\left(\frac{(2 p+1) ! !}{(2 p+4) ! !}\right) .
$$

De (6.20) e da Afirmação 6.1.1,

$$
A_{2}^{2 q}=\frac{8 q(2 q+3)}{3}\left(\frac{(2 q-1) ! !}{(2 q+2) ! !}\right) .
$$




\section{Prova da Afirmação 6.1.1}

Demonstração. Provaremos usando indução em $q$. Para $q=2$ a igualdade é verificada,

$$
\begin{gathered}
\frac{2 q}{3}\left(\frac{(2 q-1) ! !}{(2 q+2) ! !}\right)=\frac{4}{3} \frac{3}{6 ! !}=\frac{4}{48}=\frac{1}{12} \\
\frac{(2 q) ! !}{(2 q+1) ! !} \sum_{p=0}^{q-1} \frac{(2 p-1) ! !}{(2 p) ! !}\left(\frac{(2 p+1) ! !}{(2 p+4) ! !}\right)=\frac{4 ! !}{5 ! !}\left(\frac{1}{4 ! !}+\frac{1}{2} \frac{3}{(6) ! !}\right)=\frac{8}{15}\left(\frac{1}{8}+\frac{3}{96}\right)=\frac{8}{15} \frac{5}{32}=\frac{1}{12}
\end{gathered}
$$

Supondo que vale para que $q=k$,

$$
\frac{2 k}{3}\left(\frac{(2 k-1) ! !}{(2 k+2) ! !}\right)=\frac{(2 k) ! !}{(2 k+1) ! !} \sum_{p=0}^{k-1} \frac{(2 p-1) ! !}{(2 p) ! !}\left(\frac{(2 p+1) ! !}{(2 p+4) ! !}\right)
$$

vamos mostrar que vale para $q=k+1$, ou seja,

$$
\frac{2(k+1)}{3}\left(\frac{(2 k+1) ! !}{(2 k+4) ! !}\right)=\frac{(2 k+2) ! !}{(2 k+3) ! !} \sum_{p=0}^{k} \frac{(2 p-1) ! !}{(2 p) ! !}\left(\frac{(2 p+1) ! !}{(2 p+4) ! !}\right) .
$$

De fato,

$$
\begin{aligned}
\frac{(2 k+2) ! !}{(2 k+3) ! !} \sum_{p=0}^{k} \frac{(2 p-1) ! !}{(2 p) ! !}\left(\frac{(2 p+1) ! !}{(2 p+4) ! !}\right) & =\frac{(2 k+2)(2 k) ! !}{(2 k+3)(2 k+1) ! !} \sum_{p=0}^{k-1} \frac{(2 p-1) ! !}{(2 p) ! !}\left(\frac{(2 p+1) ! !}{(2 p+4) ! !}\right) \\
& +\frac{(2 k+2)}{(2 k+3)} \frac{(2 k) ! !}{(2 k+1) ! !} \frac{(2 k-1) ! !}{(2 k) ! !} \frac{(2 k+1) ! !}{(2 k+4) ! !}
\end{aligned}
$$

Usando a hipótese de indução,

$$
\begin{aligned}
\sum_{p=0}^{k} \frac{(2 p-1) ! !}{(2 p) ! !}\left(\frac{(2 p+1) ! !}{(2 p+4) ! !}\right) & =\frac{(2 k+2)}{(2 k+3)}\left(\frac{2 k}{3} \frac{(2 k-1) ! !}{(2 k+2) ! !}\right)+\frac{(2 k+2)}{(2 k+3)}\left(\frac{(2 k-1) ! !}{(2 k+4) ! !}\right) \\
& =\frac{(2 k+2)}{(2 k+3)} \frac{(2 k-1) ! !}{3(2 k+4) ! !}[(2 k+3)(2 k+1)] \\
& =\frac{2(k+1)}{3}\left(\frac{(2 k+1) ! !}{(2 k+4) ! !}\right) .
\end{aligned}
$$

Lema 6.1.6. Para todo $q$ natural, vale

$$
B_{2}^{2 q}=+\frac{8 q \pi}{3}\left(\frac{(2 q-1) ! !}{(2 q+2) ! !}\right)
$$

Demonstração. Devemos resolver o sistema:

$$
\begin{aligned}
\ddot{u}_{2 q}(t)+u_{2 q}(t) & =-2 q \mu_{1}(t)^{2 q-1} u_{1}(t) g^{(2 q-1)}(0) \\
& -2 q \mu_{1}(t) \bar{u}_{2 q-1}(t) g^{\prime}(0) .
\end{aligned}
$$




$$
u_{2 q}(0)=0, \quad \dot{u}_{2 q}(0)=0,
$$

em que $\bar{u}_{2 q-1}(t)$ é a solução do sistema

$$
\begin{gathered}
\ddot{\bar{u}}_{2 q-1}(t)+\bar{u}_{2 q-1}(t)=u_{0}(t) \mu_{1}(t)^{2 q-1} g^{(2 q-1)}(0) \\
\bar{u}_{2 q-1}(0)=0, \quad \dot{\bar{u}}_{2 q-1}(0)=0
\end{gathered}
$$

Substituindo os valores de $u_{0}(t)$ e $\mu_{1}(t)$ em (6.23), obtemos

$$
\begin{aligned}
\ddot{\bar{u}}_{2 q-1}(t)+u_{2 q-1}(t) & =-\sin (t) \cos (t)^{2 q-1}(t) g^{(2 q-1)}(0) \\
\bar{u}_{2 q-1}(0) & =0, \quad \dot{\bar{u}}_{2 q-1}(0)=0
\end{aligned}
$$

cuja solução é

$$
\bar{u}_{2 q-1}(t)=g^{(2 q-1)}(0)\left[\left(\frac{(2 q-2) ! !}{(2 q-1) ! !}-\frac{(2 q) ! !}{(2 q+1) ! !}\right) \sin (t) \sum_{p=0}^{q-1} \frac{(2 p-1) ! !}{(2 p) ! !} \cos (t)^{2 p+1}-\frac{1}{q+1} \sin (t)\right] .
$$

Substituindo os valores de $u_{1}(t), \mu_{1}(t)$ e $\bar{u}_{2 q-1}(t)$ em (6.22)

$$
\begin{aligned}
\ddot{u}_{2 q}(t)+u_{2 q}(t) & =-2 q \cos (t)^{(2 q-1)}\left(\frac{1}{3} \cos (t) \sin (t)-\frac{1}{3} \sin (t)\right) g^{\prime}(0) g^{(2 q-1)}(0) \\
& -2 q \cos (t)\left[\left(\frac{(2 q-2) ! !}{(2 q-1) ! !}-\frac{(2 q) ! !}{(2 q+1) ! !}\right) \sin (t) \sum_{p=0}^{q-1} \frac{(2 p-1) ! !}{(2 p) ! !} \cos (t)^{2 k+1}\right. \\
& \left.-\frac{1}{q+1} \sin (t)\right] g^{\prime}(0) g^{(2 q-1)}(0) .
\end{aligned}
$$

Utilizando o Método da Variação dos Parâmetros, obtemos $u_{2 q}(t)$ e fazendo $t=2 \pi$,

$$
\begin{aligned}
B_{2}^{2 q} & =4 q \pi\left(\frac{(2 q-2) ! !}{(2 q-1) ! !}-\frac{(2 q) ! !}{(2 q+1) ! !}\right) \sum_{p=0}^{q-1} \frac{(2 p-1) ! !}{(2 p) ! !}\left(\frac{(2 p+1) ! !}{(2 p+2) ! !}-\frac{(2 p+3) ! !}{(2 p+4) ! !}\right) \\
& +\frac{4 q \pi}{3}\left(\frac{(2 q-1) ! !}{(2 q) ! !}-\frac{(2 q+1) ! !}{(2 q+2) ! !}\right)
\end{aligned}
$$

Simplificando,

$$
B_{2}^{2 q}=4 q \pi\left(\frac{(2 q-2) ! !}{(2 q+1) ! !}\right) \sum_{p=0}^{q-1} \frac{(2 p-1) ! !}{(2 p) ! !} \frac{(2 p+1) ! !}{(2 p+4) ! !}+\frac{4 q \pi}{3}\left(\frac{(2 q-1) ! !}{(2 q+2) ! !}\right) .
$$

Para completar a prova do lema, utilizaremos:

Afirmação 6.1.2. Para todo natural $q \geq 2$, vale

$$
\frac{1}{3}\left(\frac{(2 q-1) ! !}{(2 q+2) ! !}\right)=\frac{(2 q-2) ! !}{(2 q+1) ! !} \sum_{p=0}^{q-1} \frac{(2 p-1) ! !}{(2 p) ! !}\left(\frac{(2 p+1) ! !}{(2 p+4) ! !}\right)
$$


Da Afirmação (6.1.2) e de (6.24), segue que

$$
B_{2}^{2 q}=+\frac{8 q \pi}{3}\left(\frac{(2 q-1) ! !}{(2 q+2) ! !}\right) .
$$

\section{Prova da Afirmação B.4.1}

Demonstração. Provaremos por indução. Para $q=2$ a igualdade é verificada,

$$
\begin{gathered}
\frac{1}{3}\left(\frac{(2 q-1) ! !}{(2 q+2) ! !}\right)=\frac{1}{3} \frac{3}{6 ! !}=\frac{1}{48} \\
\frac{(2 q-2) ! !}{(2 q+1) ! !} \sum_{p=0}^{q-1} \frac{(2 p-1) ! !}{(2 p) ! !}\left(\frac{(2 p+1) ! !}{(2 p+4) ! !}\right)=\frac{2 ! !}{5 ! !}\left(\frac{1}{4 ! !}+\frac{1}{2} \frac{3}{(6) ! !}\right)=\frac{2}{15}\left(\frac{1}{8}+\frac{3}{96}\right)=\frac{2}{15} \frac{5}{32}=\frac{1}{48}
\end{gathered}
$$

Supondo que vale para que $q=k$,

$$
\frac{1}{3}\left(\frac{(2 k-1) ! !}{(2 k+2) ! !}\right)=\frac{(2 k-2) ! !}{(2 k+1) ! !} \sum_{p=0}^{k-1} \frac{(2 p-1) ! !}{(2 p) ! !}\left(\frac{(2 p+1) ! !}{(2 p+4) ! !}\right),
$$

vamos mostrar que vale para $q=k+1$, ou seja,

$$
\frac{1}{3}\left(\frac{(2 k+1) ! !}{(2 k+4) ! !}\right)=\frac{(2 k) ! !}{(2 k+3) ! !} \sum_{p=0}^{k} \frac{(2 p-1) ! !}{(2 p) ! !}\left(\frac{(2 p+1) ! !}{(2 p+4) ! !}\right) .
$$

De fato,

$$
\begin{aligned}
\frac{(2 k) ! !}{(2 k+3) ! !} \sum_{p=0}^{k} \frac{(2 p-1) ! !}{(2 p) ! !}\left(\frac{(2 p+1) ! !}{(2 p+4) ! !}\right) & =\frac{(2 k)(2 k-2) ! !}{(2 k+3)(2 k+1) ! !} \sum_{p=0}^{k-1} \frac{(2 p-1) ! !}{(2 p) ! !}\left(\frac{(2 p+1) ! !}{(2 p+4) ! !}\right) \\
& +\frac{(2 k) ! !(2 k-1) ! !(2 k+1) ! !}{(2 k+3) ! !(2 k) ! !(2 k+4) ! !}
\end{aligned}
$$

Usando a hipótese de indução,

$$
\begin{aligned}
\frac{(2 k) ! !}{(2 k+3) ! !} \sum_{p=0}^{k} \frac{(2 p-1) ! !}{(2 p) ! !}\left(\frac{(2 p+1) ! !}{(2 p+4) ! !}\right) & =\frac{(2 k)}{(2 k+3)}\left(\frac{1}{3} \frac{(2 k-1) ! !}{(2 k+2) ! !}\right)+\left(\frac{(2 k-2) ! !(2 k-1) ! !}{(2 k) ! !(2 k+4) ! !}\right) \\
& =\frac{(2)}{(2 k+3)}\left(\frac{(2 k-1) ! !}{3(2 k+2) ! !}+\frac{(2 k-1) ! !}{2 k(2 k+4) ! !}\right) \\
& =\frac{(2 k-1) ! !}{3(2 k+3)(2 k+4) ! !}[(2 k+3)(2 k+1)] \\
& =\frac{1}{3} \frac{(2 k+1) ! !}{(2 k+4) ! !}
\end{aligned}
$$


Demonstração. Substituindo (6.13) e (6.15) na expressão de $C^{2 q}$ dada por (6.11), temos

$$
\begin{aligned}
C^{(2 q)} & =\frac{\frac{4 \pi}{3} a_{2 q}(2 q+3)\left(\frac{(2 q-1) ! !}{(2 q+2) ! !}\right)}{2 \pi \frac{(2 q+1) ! !}{(2 q+2) ! !}}-\frac{\frac{4}{3} a_{2 q}(2 q+5)\left(\frac{(2 q-3) ! !}{(2 q+2) ! !}\right)}{2 \pi \frac{(2 q-1) ! !}{(2 q+2) ! !}} \\
& =\frac{2}{3} a_{2 q}(2 q+3) \frac{(2 q-1) ! !}{(2 q+2) ! !} \frac{(2 q+2) ! !}{(2 q+1) ! !}-\frac{2}{3} a_{2 q}(2 q+5) \frac{(2 q-3) ! !}{(2 q+2) ! !} \frac{(2 q+2) ! !}{(2 q-1) ! !} \\
& -\frac{2}{3} a_{2 q}(2 q+3) \frac{(2 q-1) ! !}{(2 q+1) ! !}-\frac{2}{3} a_{2 q}(2 q+5) \frac{(2 q-3) ! !}{(2 q-1) ! !} \\
& =\frac{2}{3} a_{2 q}\left(\frac{(2 q+3)}{(2 q+1)}-\frac{(2 q+5)}{(2 q-1)}\right) \\
& =-\frac{16}{3} a_{2 q} \frac{(q+1)}{(2 q+1)(2 q-1)} .
\end{aligned}
$$

Agora, substituindo (6.17) e (6.21) na expressão de $D^{2 q}$ dada por (6.12), temos

$$
\begin{aligned}
D^{2 q} & =\frac{\frac{8 q \pi}{3}(2 q+3)\left(\frac{(2 q-1) ! !}{(2 q+2) ! !}\right)}{2 \pi\left(\frac{(2 q+1) ! !}{(2 q+2) ! !}\right)}+\frac{\frac{8 q \pi}{3}\left(\frac{(2 q-1) ! !}{(2 q+2) ! !}\right)}{2 \pi\left(\frac{(2 q-1) ! !}{(2 q+2) ! !}\right)} \\
& =\frac{4 q}{3}(2 q+3)\left(\frac{(2 q-1) ! !}{(2 q+1) ! !}\right)+\frac{4 q}{3} \\
& =\frac{16 q}{3}\left(\frac{(q+1)}{(2 q+1)}\right) .
\end{aligned}
$$

Como $a_{2 q}=q(2 q-1)$, concluímos que

$$
D^{(2 q)}=-C^{(2 q)}=\frac{16 q}{3}\left(\frac{(q+1)}{(2 q+1)}\right), \quad \forall q \geq 2 \in \mathbb{N} .
$$


RESULTADO PRINCIPAL 


\section{Capítulo 7}

\section{Comentários e Aplicações}

Neste capítulo apresentaremos algumas aplicações da teoria desenvolvida nos capítulos anteriores. Inicialmente, na Seção 7.1, provamos dois corolários do Teorema 5.1.2, que fornecem condições necessárias para que o sistema (5.1) possua $\tau\left(x_{0}\right)$-isocronismo.

Na Seção 7.2, utilizando esses corolários, reobtemos alguns dos resultados para estabilidade de sistemas quase-centrais apresentados no Capítulo 3.

Para finalizar, na Seção 7.3, obtemos uma extensão do Teorema 4.1.2, para o caso $n=2$.

Ao menos de menção explícita ao contrário, estamos supondo $f$ e $g$ positivas e definidas numa vizinhança de $0 \in \mathbb{R}$.

\subsection{Condições necessárias para o $\tau\left(x_{0}\right)$-isocronismo}

Nesta seção, supondo as funções $f$ e $g$ de classe $C^{4}$, obtemos condições necessárias em função das derivadas de $g$ e $f$ em zero até a quarta ordem para que o sistema 5.1 possua $\tau\left(x_{0}\right)$-isocronismo.

Dividiremos em dois casos, quando $3 f^{\prime \prime}(0)>4 f^{\prime}(0)^{2}$ e $3 f^{\prime}(0)=4 f^{\prime}(0)^{2}$.

Corolário 7.1.1. Supondo $f$ e $g$ de classe $C^{4}$ satisfazendo $f(0)=g(0)=1$ e $3 f^{\prime \prime}(0)>4 f^{\prime}(0)^{2}$. Se (5.1) possui $\tau\left(x_{0}\right)$-isocronismo, então

$$
\begin{aligned}
& g^{\prime}(0)=f^{\prime}(0) \pm \frac{1}{4} \sqrt{-24 f^{\prime}(0)^{2}+18 f^{\prime \prime}(0)}, \\
& g^{\prime \prime}(0)=-\frac{20}{9} f^{\prime}(0)^{2}+f^{\prime \prime}(0)+\frac{10}{9} f^{\prime}(0) g^{\prime}(0)+\frac{10}{9} g^{\prime}(0)^{2}, \\
& g^{(3)}(0)\left(f^{\prime}(0)-g^{\prime}(0)\right)=-\frac{94}{48} g^{\prime}(0)^{4}+2 g^{\prime}(0) f^{(3)}(0)+\frac{781}{24} g^{\prime}(0) f^{\prime}(0)^{3}+\frac{47}{24} g^{\prime}(0)^{3} f^{\prime}(0)-\frac{725}{48} g^{\prime}(0)^{2} f^{\prime}(0)^{2} \\
& \text { - } 7 f^{\prime}(0) f^{(3)}(0)+\frac{5}{8} f^{(4)}(0)-\frac{395}{4} f^{\prime}(0)^{4}+\frac{515}{8} f^{\prime}(0)^{2} f^{\prime \prime}(0)-\frac{171}{64} f^{\prime \prime}(0)^{2}-\frac{21}{64} g^{\prime \prime}(0)^{2} \\
& +\frac{155}{48} g^{\prime}(0) g^{\prime \prime}(0) f^{\prime}(0)-\frac{579}{32} g^{\prime}(0) f^{\prime \prime}(0) f^{\prime}(0)-\frac{59}{16} g^{\prime \prime}(0) f^{\prime}(0)^{2}+\frac{121}{32} g^{\prime}(0)^{2} f^{\prime \prime}(0) \\
& -\frac{33}{64} g^{\prime \prime}(0) f^{\prime \prime}(0)+\frac{109}{48} g^{\prime}(0)^{2} g^{\prime \prime}(0) \text {, }
\end{aligned}
$$




$$
\begin{aligned}
g^{(4)}(0) & =-\frac{56}{5} f^{\prime}(0) f^{(3)}(0)+55 f^{\prime}(0)^{2} f^{\prime \prime}(0)-\frac{171}{40} f^{\prime \prime}(0)^{2}-\frac{154}{3} f^{\prime}(0)^{4}+f^{(4)}(0) \\
& -\frac{171}{40} f^{\prime \prime}(0)^{2}+\frac{28}{5} g^{(3)}(0) f^{\prime}(0)+\frac{28}{15} g^{\prime}(0) f^{(3)}(0)-\frac{39}{20} g^{\prime \prime}(0) f^{\prime \prime}(0)+\frac{89}{5} g^{\prime}(0)^{3} f^{\prime}(0) \\
& -\frac{91}{15} g^{\prime \prime}(0) f^{\prime}(0)^{2}+\frac{56}{15} g^{\prime}(0) g^{(3)}(0)+\frac{43}{10} g^{\prime}(0)^{2} f^{\prime \prime}(0)-\frac{29}{6} g^{\prime}(0)^{2} f^{\prime}(0)^{2}-\frac{446}{15} g^{\prime}(0) f^{\prime}(0)^{3} \\
& -\frac{551}{30} g^{\prime}(0)^{2} g^{\prime \prime}(0)-\frac{115}{6} g^{\prime}(0) g^{\prime \prime}(0) f^{\prime}(0)-\frac{157}{10} g^{\prime}(0) f^{\prime \prime}(0) f^{\prime}(0)+\frac{259}{30} g^{\prime}(0)^{4}-\frac{249}{40} g^{\prime \prime}(0)^{2} .
\end{aligned}
$$

Demonstração. Vamos aplicar o Teorema 5.1.2.

Para $k=2$, os valores de $g^{\prime}(0)$ e $g^{\prime \prime}(0)$ são dados no Teorema 6.1.1, ou seja,

$$
\begin{gathered}
g^{\prime}(0)=f^{\prime}(0) \pm \frac{1}{4} \sqrt{-24 f^{\prime}(0)^{2}+18 f^{\prime \prime}(0)}, \\
g^{\prime \prime}(0)=\frac{9 f^{\prime \prime}(0)}{4} \pm \frac{5 f^{\prime}(0)}{6} \sqrt{-24 f^{\prime}(0)^{2}+18 f^{\prime \prime}(0)}-\frac{5 f^{\prime}(0)^{2}}{3} .
\end{gathered}
$$

Como $3 f^{\prime \prime}(0)>4 f^{\prime}(0)^{2}$, então $g^{\prime}(0)$ e $g^{\prime \prime}(0)$ estão bem definidos.

Para $k$ ímpar, ao aplicarmos o Teorema 5.1.2 não obtemos informaçoes sobre $g^{k}(0)$. Portanto, aplicaremos somente para as derivadas de ordem par.

Para $k=4$, utilizaremos as fórmulas dadas no Teorema 5.2.1.

Calculando $\tau^{(4)}(0)-t_{1}^{(4)}(0)=0$ e isolando $g^{(4)}(0)$, obtemos

$$
\begin{aligned}
g^{(4)}(0) & =-\frac{56}{5} f^{\prime}(0) f^{(3)}(0)+55 f^{\prime}(0)^{2} f^{\prime \prime}(0)-\frac{171}{40} f^{\prime \prime}(0)^{2}-\frac{154}{3} f^{\prime}(0)^{4}+f^{(4)}(0) \\
& -\frac{171}{40} f^{\prime \prime}(0)^{2}+\frac{28}{5} g^{(3)}(0) f^{\prime}(0)+\frac{28}{15} g^{\prime}(0) f^{(3)}(0)-\frac{39}{20} g^{\prime \prime}(0) f^{\prime \prime}(0)+\frac{89}{5} g^{\prime}(0)^{3} f^{\prime}(0) \\
& -\frac{91}{15} g^{\prime \prime}(0) f^{\prime}(0)^{2}+\frac{56}{15} g^{\prime}(0) g^{(3)}(0)+\frac{43}{10} g^{\prime}(0)^{2} f^{\prime \prime}(0)-\frac{29}{6} g^{\prime}(0)^{2} f^{\prime}(0)^{2}-\frac{446}{15} g^{\prime}(0) f^{\prime}(0)^{3} \\
& -\frac{551}{30} g^{\prime}(0)^{2} g^{\prime \prime}(0)-\frac{115}{6} g^{\prime}(0) g^{\prime \prime}(0) f^{\prime}(0)-\frac{157}{10} g^{\prime}(0) f^{\prime \prime}(0) f^{\prime}(0)+\frac{259}{30} g^{\prime}(0)^{4}-\frac{249}{40} g^{\prime \prime}(0)^{2} .
\end{aligned}
$$

Agora, calculando $\tau^{(4)}(0)-t_{2}^{(4)}(0)$ e isolando $g^{(4)}(0)$, temos

$$
\begin{aligned}
g^{(4)}(0) & =-56 f^{\prime}(0) f^{(3)}(0)+275 f^{\prime}(0)^{2} f^{\prime \prime}(0)+5 f^{(4)}(0)-\frac{730}{3} f^{\prime}(0)^{4}-\frac{171}{8} f^{\prime \prime}(0)^{2} \\
& +\frac{33}{8} g^{\prime \prime}(0)^{2}-\frac{7}{2} g^{\prime}(0)^{4}-\frac{8}{3} g^{\prime}(0) g^{(3)}(0)+\frac{57}{2} g^{\prime}(0)^{2} f^{\prime \prime}(0)-\frac{21}{4} g^{\prime \prime}(0) f^{\prime \prime}(0) \\
& +238 g^{\prime}(0) f^{\prime}(0)^{3}-\frac{203}{2} g^{\prime}(0)^{2} f^{\prime}(0)^{2}+\frac{91}{3} g^{\prime}(0)^{3} f^{\prime}(0)-\frac{89}{3} g^{\prime \prime}(0) f^{\prime}(0)^{2}-\frac{23}{6} g^{\prime}(0)^{2} g^{\prime \prime}(0) \\
& +\frac{44}{3} g^{\prime}(0) f^{\prime \prime \prime}(0)+12 g^{(3)}(0) f^{\prime}(0)-\frac{263}{2} g^{\prime}(0) f^{\prime \prime}(0) f^{\prime}(0)+\frac{3}{2} g^{\prime}(0) g^{\prime \prime}(0) f^{\prime}(0)
\end{aligned}
$$

Igualando as duas expressões acima e isolando $g^{(3)}(0)$, 


$$
\begin{aligned}
g^{(3)}(0)\left(f^{\prime}(0)-g^{\prime}(0)\right) & =+\frac{91}{48} g^{\prime}(0)^{4}-2 g^{\prime}(0) f^{(3)}(0)-\frac{781}{24} g^{\prime}(0) f^{\prime}(0)^{3}-\frac{47}{24} g^{\prime}(0)^{3} f^{\prime}(0)+\frac{725}{48} g^{\prime}(0)^{2} f^{\prime}(0)^{2} \\
& +7 f^{\prime}(0) f^{(3)}(0)-\frac{5}{8} f^{(4)}(0)+\frac{385}{12} f^{\prime}(0)^{4}-\frac{275}{8} f^{\prime}(0)^{2} f^{\prime \prime}(0)+\frac{171}{64} f^{\prime \prime}(0)^{2}+\frac{21}{64} g^{\prime \prime}(0)^{2} \\
& -\frac{155}{48} g^{\prime}(0) g^{\prime \prime}(0) f^{\prime}(0)+\frac{579}{32} g^{\prime}(0) f^{\prime \prime}(0) f^{\prime}(0)+\frac{59}{16} g^{\prime \prime}(0) f^{\prime}(0)^{2}-\frac{121}{32} g^{\prime}(0)^{2} f^{\prime \prime}(0) \\
& +\frac{33}{64} g^{\prime \prime}(0) f^{\prime \prime}(0)-\frac{109}{48} g^{\prime}(0)^{2} g^{\prime \prime}(0) .
\end{aligned}
$$

Podemos escolher uma das duas fórmulas para $g^{(4)}(0)$, optamos pela primeira.

Observe que temos duas condições distintas para $g^{\prime}(0)$, para cada uma dessas condições temos uma condição diferente para $g^{\prime \prime}(0)$, e assim sucessivamente. Logo, obtemos duas condições distintas para cada derivada de $g(x)$ calculada em zero.

Corolário 7.1.2. Supondo $f$ e $g$ de classe $C^{4}$ satisfazendo $f(0)=g(0)=1$ e $3 f^{\prime \prime}(0)=4 f^{\prime}(0)^{2}$. Se (5.1) possui $\tau\left(x_{0}\right)$-isocronismo, então

$$
\begin{gathered}
g^{\prime}(0)=f^{\prime}(0), \quad g^{\prime \prime}(0)=f^{\prime \prime}(0), \\
g^{(4)}(0)=-\frac{4}{3} f^{\prime}(0) f^{(3)}(0)-\frac{40}{3} f^{\prime}(0)^{4}+\frac{28}{3} g^{(3)}(0) f^{\prime}(0) .
\end{gathered}
$$

Demonstração. Vamos aplicar o Teorema 5.1.2.

Para $k=2$, os valores de $g^{\prime}(0)$ e $g^{\prime \prime}(0)$ são dados no Teorema 6.1.1, ou seja,

$$
\begin{gathered}
g^{\prime}(0)=f^{\prime}(0) \pm \frac{1}{4} \sqrt{-24 f^{\prime}(0)^{2}+18 f^{\prime \prime}(0)}, \\
g^{\prime \prime}(0)=\frac{9 f^{\prime \prime}(0)}{4} \pm \frac{5 f^{\prime}(0)}{6} \sqrt{-24 f^{\prime}(0)^{2}+18 f^{\prime \prime}(0)}-\frac{5 f^{\prime}(0)^{2}}{3} .
\end{gathered}
$$

Usando que $3 f^{\prime \prime}(0)=4 f^{\prime}(0)^{2}$, segue que:

$$
g^{\prime}(0)=f^{\prime}(0), \quad g^{\prime \prime}(0)=f^{\prime \prime}(0) .
$$

Para $k>2$, as contas são as mesmas da demonstração do corolário anterior. Assim, na fórmula de $g^{(3)}(0)$, usando que $g^{\prime}(0)=f^{\prime}(0)$ e $g^{\prime \prime}(0)=f^{\prime \prime}(0)=\frac{4}{3} f^{\prime}(0)^{2}$, obtemos

$$
f^{(4)}(0)=-\frac{40}{3} f^{\prime}(0)^{(4)}+8 f^{\prime}(0) f^{(3)}(0)
$$

Na expressão de $g^{(4)}(0)$, usando que $g^{\prime}(0)=f^{\prime}(0), \quad 3 g^{\prime \prime}(0)=3 f^{\prime \prime}(0)=4 f^{\prime}(0)^{2}$ e (7.1), temos

$$
g^{(4)}(0)=-\frac{4}{3} f^{\prime}(0) f^{(3)}(0)-\frac{40}{3} f^{\prime}(0)^{4}+\frac{28}{3} g^{(3)}(0) f^{\prime}(0)
$$

Observamos que não há restrição sobre $g^{(3)}(0)$. O mesmo se mantêm para todas as derivadas ímpares de $g(x)$ em zero de ordem maior que 3 . 


\subsection{Aplicações}

Nesta seção apresentamos algumas aplicações dos Corolários 7.1.1 e 7.1.2. Nosso objetivo é mostrar como os nossos resultados estão relacionados com resultados já conhecidos de estabilidade para sistema de forças quase-centrais.

\subsubsection{Caso $f=g$}

Considere o sistema

$$
\left\{\begin{array}{l}
\ddot{x}=-x f(x), \\
\ddot{y}=-y f(x),
\end{array}\right.
$$

com $f$ definida numa vizinhança da origem $I$, positiva e de classe $C^{4}$.

Como vimos, no Capítulo 3, a estabilidade da origem de sistemas do tipo (7.2) foi estudada em [BNZ86],[BNdOC88] e [Zam88]. Nestes trabalhos mostrou-se que a origem do sistema é estável se e somente se cada solução perto da origem é periódica de menor período $\tau\left(x_{0}\right)$. Logo, a origem do sistema é estável se e somente se o sistema possui $\tau\left(x_{0}\right)$-isocronismo.

Existem funções não triviais em que a origem de (7.2) é estável. Por exemplo, no artigo [BNdOC88] é exibida a seguinte família de funções para as quais a origem é estável (veja o Capítulo $3)$ :

$$
f(x)=(p+q x)^{-3}, \quad p \in \mathbb{R}_{+}^{*}, q \in \mathbb{R} .
$$

No que segue, encontraremos condições necessárias para estabilidade da origem de (7.2), com $f(0)=1$. Iremos reobter a "velha" condição de estabilidade, demonstrada originalmente em [BNdOC88], $3 f^{\prime \prime}(0)^{2}=4 f^{\prime}(0)^{2}$. Além disso, apresentaremos outra condição de estabilidade para $f^{(4)}(0)$, dada em função das derivadas $f^{\prime}(0)$ e $f^{(3)}(0)$.

Aplicação 7.2.1. Se a origem do sistema (7.2) é estável, então

$$
\begin{gathered}
f^{\prime \prime}(0)=\frac{4}{3} f^{\prime}(0)^{2}, \\
f^{(4)}(0)=-\frac{40}{3} f^{\prime}(0)^{4}+8 f^{(3)}(0) f^{\prime}(0) .
\end{gathered}
$$

Demonstração. Como a origem de (7.2) é estável, então o sistema possui $\tau\left(x_{0}\right)$-isocronismo e as seguintes condições são satisfeitas:

$$
\begin{gathered}
g^{\prime}(0)=f^{\prime}(0) \pm \frac{1}{4} \sqrt{-24 f^{\prime}(0)^{2}+18 f^{\prime \prime}(0)} \\
g^{\prime \prime}(0)=\frac{9 f^{\prime \prime}(0)}{4} \pm \frac{5 f^{\prime}(0)}{6} \sqrt{-24 f^{\prime}(0)^{2}+18 f^{\prime \prime}(0)}-\frac{5 f^{\prime}(0)^{2}}{3} .
\end{gathered}
$$

Usando que $f^{\prime}(0)=g^{\prime}(0)$ e $g^{\prime \prime}(0)=f^{\prime \prime}(0)$, obtemos

$$
3 f^{\prime \prime}(0)=4 f^{\prime}(0)^{2} .
$$

Estamos nas hipóteses do Corolário 7.1.2, logo

$$
g^{(4)}(0)=-\frac{4}{3} f^{\prime}(0) f^{(3)}(0)-\frac{40}{3} f^{\prime}(0)^{4}+\frac{28}{3} g^{(3)}(0) f^{\prime}(0) .
$$


Como $g^{(3)}(0)=f^{(3)}(0)$ e $g^{(4)}(0)=f^{(4)}(0)$, segue que

$$
f^{(4)}(0)=8 f^{\prime}(0) f^{(3)}(0)-\frac{40}{3} f^{\prime}(0)^{4} .
$$

Exemplo 7.2.1. Considere o sistema (7.2), com $f(x)=(1+q x)^{3}, q \in \mathbb{R}$.

Sabemos que a origem do sistema é estável. Vamos verificar que as condições (7.3) e (7.4) são satisfeitas.

De fato, temos que

$$
f(0)=1, f^{\prime}(0)=-3 q, \quad f^{\prime \prime}(0)=12 q^{2}, \quad f^{(3)}(0)=-60 q^{3}, \quad f^{(4)}(0)=360 q^{4},
$$

$\log o$

$$
f^{\prime \prime}(0)=12 q^{2}=\frac{4(3 q)^{2}}{3}=\frac{4 f^{\prime}(0)^{2}}{3} .
$$

Desenvolvendo o lado direito de (7.4), temos

$$
8 f^{\prime}(0) f^{(3)}(0)-\frac{40}{3} f^{\prime}(0)^{4}=8(-3 q)\left(-60 q^{3}\right)-\frac{40}{3}(-3 q)^{4}=1440 q^{4}-1080 q^{4}=360 q^{4}=f^{(4)}(0) .
$$

\subsubsection{Centro isócrono}

Considere o sistema

$$
\left\{\begin{array}{l}
\ddot{x}=-x f(x) \\
\ddot{y}=-y\left(f(x)+x f^{\prime}(x)\right) .
\end{array}\right.
$$

Como vimos na Seção 4.2 a origem do sistema (7.5) é estável se, e somente se, o centro do sistema planar $\ddot{x}=-x f(x)$ é isócrono. Neste caso, todas as soluções de 7.5 numa vizinhança da origem em $\mathbb{R}^{4}$ são períodicas de período $\tau\left(x_{0}\right)$.

Concluímos que se o sistema (7.5) possui $\tau\left(x_{0}\right)$ - isocronismo, então a origem em $\mathbb{R}^{4}$ é estável, logo o centro $\ddot{x}=-x f(x)$ é isócrono. Assim, como consequência do Corolário 4.2.1, as seguintes condições devem ser satisfeitas:

$$
f^{\prime \prime}(0)=\frac{20 f^{\prime}(0)^{2}}{9}, \quad f^{(4)}(0)=\frac{56}{5} f^{(3)}(0) f^{\prime}(0)-\frac{448 f^{\prime}(0)^{4}}{9} .
$$

No entanto, a recíproca não é necessariamente verdadeira, ou seja, se a origem de (7.5) é estável, não podemos afirmar que o sistema possui $\tau\left(x_{0}\right)$-isocronismo, uma vez que todas as soluções perto da origem em $\mathbb{R}^{4}$ podem ser periódicas de período $\tau\left(x_{0}\right)$, mas o menor período destas soluções pode ser diferente de $\tau\left(x_{0}\right)$.

No que segue, mostramos que se o sistema (7.5) possui $\tau\left(x_{0}\right)$ - isocronismo, então as condições (7.6) são verificadas.

Aplicação 7.2.2. Considere o sistema (7.5), com $f$ de classe $C^{4}$ e $f(0)=1$. Se (7.5) possui $\tau\left(x_{0}\right)$ isocronismo, então

$$
f^{\prime \prime}(0)=\frac{20}{9} f^{\prime}(0)^{2}, \quad f^{4}(0)=-\frac{448}{9} f^{\prime}(0)^{4}+\frac{56}{5} f^{(3)}(0) f^{\prime}(0) .
$$


Demonstração. Por hipótese o sistema possui $\tau\left(x_{0}\right)$-isocronismo, logo as seguintes condições são satisfeitas:

$$
\begin{gathered}
g^{\prime}(0)=f^{\prime}(0) \pm \frac{1}{4} \sqrt{-24 f^{\prime}(0)^{2}+18 f^{\prime \prime}(0)}, \\
g^{\prime \prime}(0)=\frac{9 f^{\prime \prime}(0)}{4} \pm \frac{5 f^{\prime}(0)}{6} \sqrt{-24 f^{\prime}(0)^{2}+18 f^{\prime \prime}(0)}-\frac{5 f^{\prime}(0)^{2}}{3} .
\end{gathered}
$$

Como $g(x)=f(x)+x f^{\prime}(x)$, então $g^{\prime}(0)=2 f^{\prime}(0)$ e $g^{\prime \prime}(0)=3 f^{\prime \prime}(0)$. Substituindo nas condições dadas acima, obtemos

$$
f^{\prime \prime}(0)=\frac{20}{9} f^{\prime}(0)^{2}
$$

Estamos nas hipóteses do Corolário 7.1.1. Substituindo $g^{(3)}(0)=4 f^{(3)}(0)$ e $g^{(4)}(0)=5 f^{(4)}(0)$ nas fórmulas dadas nesse corolário, temos

$$
f^{4}(0)=-\frac{448}{9} f^{\prime}(0)^{4}+\frac{56}{5} f^{\prime \prime \prime}(0) f^{\prime}(0) .
$$

Agora, supondo que o centro do sistema $\ddot{x}=-x f(x)$ seja isócrono, encontraremos condições necessárias para ocorrência de $\tau\left(x_{0}\right)$ - isocronismo em (7.5).

Aplicação 7.2.3. (Centro isócrono) Considere o sistema (5.1). Suponha $f$ e $g$ de classe $C^{4}$, com $f(0)=g(0)=1$ e tal que a origem $x=0, \dot{x}=0$ é um centro isócrono de $\ddot{x}=-x f(x)$ de período $\tau\left(x_{0}\right)$. Se (5.1) possui $\tau\left(x_{0}\right)$-isocronismo, então

$$
g^{\prime}(0)=g^{\prime \prime}(0)=g^{(3)}(0)=g^{4}(0)=0
$$

ou

$$
g^{\prime}(0)=2 f^{\prime}(0), \quad g^{\prime \prime}(0)=3 f^{\prime \prime}(0), \quad g^{(3)}(0)=4 f^{(3)}(0), \quad g^{(4)}(0)=5 f^{(4)}(0)
$$

Demonstração. Por hipótese, o centro de $\ddot{x}=-x f(x)$ é isócrono, logo

$$
f^{\prime \prime}(0)=\frac{20}{9} f^{\prime}(0)^{2}, \quad f^{4}(0)=-\frac{448}{9} f^{\prime}(0)^{4}+\frac{56}{5} f^{\prime \prime \prime}(0) f^{\prime}(0) .
$$

Como $f^{\prime \prime}(0)=\frac{20}{9} f^{\prime}(0)^{2}$, as hipóteses do Corolário (7.1.1) são satisfeitas, substituindo os valores dados acima nas fórmulas de $g^{\prime}(0), g^{\prime \prime}(0), g^{\prime \prime \prime}(0)$ e $g^{(4)}(0)$, o resultado segue.

\subsubsection{Paridade da função $f(x)$ e $\tau\left(x_{0}\right)$-isocronismo}

Aplicação 7.2.4. Considere o sistema (5.1). Supondo $f(x)$ e $g(x)$ de classe $C^{4}$, com $f(0)=g(0)=$ 1. $e f(x)$ par.

a) Se $f^{\prime \prime}(0)>0$ e (5.1) possui $\tau\left(x_{0}\right)$ - isocronismo, então

$$
g^{\prime}(0)= \pm \frac{3 \sqrt{2 f^{\prime \prime}(0)}}{4}, \quad g^{\prime \prime}(0)=\frac{9 f^{\prime \prime}(0)^{2}}{4}
$$




$$
\begin{aligned}
g^{(3)}(0) g^{\prime}(0) & =\frac{5}{8} f^{(4)}(0)-\frac{171}{64} f^{\prime \prime}(0)^{2}-\frac{91}{48} g^{\prime}(0)^{4}-\frac{21}{64} g^{\prime \prime}(0)^{2}+\frac{121}{32} g^{\prime}(0)^{2} f^{\prime \prime}(0) \\
& -\frac{33}{64} g^{\prime \prime}(0) f^{\prime \prime}(0)+\frac{109}{48} g^{\prime}(0)^{2} g^{\prime \prime}(0) \\
g^{(4)}(0) & =-\frac{171}{40} f^{\prime \prime}(0)^{2}+\frac{43}{10} g^{\prime}(0)^{2} f^{\prime \prime}(0)+\frac{56}{16} g^{\prime}(0) g^{(3)}(0)-\frac{39}{20} g^{\prime \prime}(0) f^{\prime \prime}(0)-\frac{551}{30} g^{\prime}(0)^{2} g^{\prime \prime}(0) \\
& +\frac{259}{30} g^{\prime}(0)^{4}+\frac{249}{40} g^{\prime \prime}(0)^{2}+f^{(4)}(0) .
\end{aligned}
$$

b) $S e f^{\prime \prime}(0)=0$ e (5.1) possui $\tau\left(x_{0}\right)$ - isocronismo, então

$$
g^{\prime}(0)=0, \quad g^{\prime \prime}(0)=0, \quad g^{(4)}(0)=0 .
$$

c) Se $f^{\prime \prime}(0)<0$, então não existe $g(x)$ real tal que (5.1) possui $\tau\left(x_{0}\right)$-isocronismo.

Demonstração. A função $f(x)$ é par, logo todas as derivadas de ordem ímpar são nulas.

Por hipótese, o sistema possui $\tau\left(x_{0}\right)$-isocronismo, utilizando as fórmulas do Teorema 6.1.1, a seguinte condição é satisfeita

$$
g^{\prime}(0)= \pm \frac{1}{4} \sqrt{18 f^{\prime \prime}(0)}
$$

Os itens a) e b) seguem, respectivamente, dos Corolários 7.1.1 e 7.1.2.

Se $f^{\prime \prime}(0)<0$, então a raiz da fórmula de $g^{\prime}(0)$ não está definida, logo não existe $g(x)$ real tal que o sistema (5.1) possui $\tau\left(x_{0}\right)$-isocronismo.

Aplicação 7.2.5. ( $f(x)+1$ impar) Supondo que $f(x)-1$ e $g(x)$ são de classe $C^{k}, k$ natural, satisfazendo $f(0)=g(0)=1$. Se $f(x)-1$ é impar, então não existe $g(x)$ real tal que o sistema (5.1) possui $\tau\left(x_{0}\right)$-isocronismo.

Para o caso $f=g$, no artigo ([BNZ86]), mostrou-se que: se a função $f(x)$ é contínua e par, a origem do sistema é estável se, e somente se, $f(x)$ é localmente constante em zero.

Na próxima aplicação, para o caso $f(x)$ analítica com $f(0)=1$, provamos esse resultado de uma maneira diferente.

Aplicação 7.2.6. Considere o sistema (7.2), com $f$ analítica, par e $f(0)=1$. A origem do sistema é estável se e somente se $f(x)$ é localmente constante em zero.

Demonstração. A condição suficiente é imediata.

Vamos provar a condição necessária. Por hipótese, a origem é estável, logo o sistema (7.2) possui $\tau\left(x_{0}\right)$ - isocronismo.

Fazendo $\tau^{(2 q)}(0)-t_{2}^{(2 q)}(0)=0$ e isolando $g^{(2 q)}(0)$, obtemos a expressão:

$$
g^{(2 q)}(0)=\frac{H_{2}^{2 q}\left[f^{\prime}(0), \ldots, f^{(2 q)}(0), g^{\prime}(0), \ldots, g^{(2 q-1)}(0)\right]}{-2 \pi\left(\frac{(2 q-1) ! !}{(2 q) ! !}-\frac{(2 q+1) ! !}{(2 q+2) ! !}\right)} .
$$

(Vide demonstração do Teorema 6.1.2.)

Note que, $f^{(2 q)}(0)$ aparece apenas na expressão de $\tau^{(4)}(0)$ e, como mostramos no Lema 5.3.1, $f^{(2 q)}(0)$ é múltiplo de $-2 \pi((2 q+1) ! ! /(2 q+2) ! !)$. Logo, em $(7.7), f^{(2 q)}(0)$ é múltiplo de $(2 q+1)$. 
Fazendo $f=g$ nessa expressão,

$$
f_{2}^{(2 q)}(0)=\frac{H_{2}^{2 q}\left[f^{\prime}(0), \ldots, f^{(2 q)}(0)\right]}{-2 \pi\left(\frac{(2 q-1) ! !}{(2 q) ! !}-\frac{(2 q+1) ! !}{(2 q+2) ! !}\right)},
$$

isolando $f^{(2 q)}(0)$ e usando que a função $f(x)$ é par, ou seja, todas as derivadas ímpares de $f(x)$ são nulas, temos

$$
(2 q) f^{(2 q)}(0)=\frac{\tilde{H}_{1}^{(2 q)}\left[f^{\prime \prime}(0), \ldots, f^{(2 q-2)}(0)\right]}{-2 \pi\left(\frac{(2 q-1) ! !}{(2 q+2) ! !}\right)} .
$$

Como o sistema possui $\tau\left(x_{0}\right)$-isocronismo, vale que $f^{\prime \prime}(0)=\frac{4}{3} f^{\prime}(0)^{2}, \log 0 f^{\prime \prime}(0)=0$.

Usando indução sobre $q$ em (7.8), concluí-se que $f^{(2 q)}(0)=0$ para todo natural $q$.

Como a função $f(x)$ é analítica com $f(0)=1$ e $f^{(k)}(0)=0$, para todo $k$ natural, concluímos que $f(x)=1$.

\subsection{Alguns resultados de estabilidade}

Esta seção destoa um pouco do resto da tese, aqui se discutem resultados de estabilidade de sistemas quase-centrais, em que o $\tau\left(x_{0}\right)$-isocronismo aparece de forma literal.

Ressolvemos incluir a seção pois (além de ela ter poucas páginas), aproveitam-se os cálculos feitos para as derivadas de $\tau, t_{1}$ e $t_{2}$ para exibir mais algumas condições computáveis de estabilidade, que não apareciam na literatura e também destaca o fato, bastante esperado, de $\tau\left(x_{0}\right)$-isocronismo, além de ser um fenômeno raro, altamente genérico, não pode ser"finitamente detectado"no sentido dado por A.Barone, M.O.Cesar e G.Gorni em [BNdOCG02].

Como vimos, em [BNdOCG02] é estudado o sistema:

$$
\left\{\begin{array}{l}
\ddot{x}=-x f(x), \\
\ddot{y}=-y g(x),
\end{array}\right.
$$

com $f, g$ funções de classe $C^{2}$ definidas numa vizinhança aberta de $0 \in \mathbb{R}$, com $f(0)>0, g(0)>0$ e $4 g(0)=n^{2} f(0)$, para algum natural $n$.

Para $x_{0}$ suficientemente pequeno é definida a função que caracteriza a estabilidade:

$$
\bar{l}\left(x_{0}\right)=\left\{\begin{array}{rrr}
\frac{\tau\left(x_{0}\right)-t_{1}\left(x_{0}\right)}{\tau\left(x_{0}\right)-t_{2}\left(x_{0}\right)} & \text { se } & t_{2}\left(x_{0}\right) \neq \tau\left(x_{0}\right) \\
1 & \text { se } & t_{1}\left(x_{0}\right)=t_{2}\left(x_{0}\right)=\tau\left(x_{0}\right) \\
+\infty & \text { se } & t_{1}\left(x_{0}\right) \neq t_{2}\left(x_{0}\right)=\tau\left(x_{0}\right) .
\end{array}\right.
$$

O Novo Critério de Estabilidade (Teorema 4.1.1) é dado em termos da função $\bar{l}\left(x_{0}\right)$. Utilizando esse critério são obtidas condições necessárias e/ou suficientes, em termos das derivadas de $f(x)$ e $g(x)$ em zero até a segunda ordem, que permitem verificar se a origem do sistema é estável ou não. Aqui, reproduziremos o resultado para o caso em que estamos interessados, ou seja, $n=2$. Para os demais valores de $n$ veja o Capítulo 4 . 
Teorema 7.3.1. Supondo que $f$ e $g$ são de classe $C^{2}$ e $g(0)=f(0)=1$. Sejam

$$
\begin{aligned}
& p_{2}:=20 f^{\prime}(0)^{2}-10 f^{\prime}(0) g^{\prime}(0)-10 g^{\prime}(0)^{2}+9\left(g^{\prime \prime}(0)-f^{\prime \prime}(0)\right), \\
& p_{3}:=20 f^{\prime}(0)^{2}-14 f^{\prime}(0) g^{\prime}(0)+2 g^{\prime}(0)^{2}+3\left(g^{\prime \prime}(0)-3 f^{\prime \prime}(0)\right),
\end{aligned}
$$

Se $p_{2} p_{3}>0$, então a origem é estável, se $p_{2} p_{3}<0$ ou somente um dos dois é nulo, então a origem é instável.

No teorema anterior, $p_{2}$ e $p_{3}$ são obtidos, respectivamente, fazendo $12\left(\tau^{(2)}(0)-t_{1}^{(2)}(0)\right)$ e $12\left(\tau^{(2)}(0)-t_{2}^{(2)}(0)\right)$.

Uma pergunta natural é: o que ocorre se $p_{2}=p_{3}=0$ ?

Utilizando as fórmulas das derivadas de $\tau, t_{1}$ e $t_{2}$ em zero até a quarta ordem, dadas no Teorema 5.2.1, é possível responder essa pergunta.

Suporemos $f$ e $g$ de classe $C^{4}$ e denotaremos $p_{2}$ por $q_{1}^{(2)}$ e $p_{3}$ por $q_{2}^{(2)}$.

Teorema 7.3.2. Supondo que $f$ e $g$ são de classe $C^{4}$ e $g(0)=f(0)=1$, sejam

$$
\begin{gathered}
q_{1}^{(2)}:=20 f^{\prime}(0)^{2}-10 f^{\prime}(0) g^{\prime}(0)-10 g^{\prime}(0)^{2}+9\left(g^{\prime \prime}(0)-f^{\prime \prime}(0)\right), \\
q_{2}^{(2)}:=20 f^{\prime}(0)^{2}-14 f^{\prime}(0) g^{\prime}(0)+2 g^{\prime}(0)^{2}+3\left(g^{\prime \prime}(0)-3 f^{\prime \prime}(0)\right), \\
q_{1}^{(4)}:=1344 f^{\prime}(0) f^{(3)}(0)-6600 f^{\prime}(0)^{2} f^{\prime \prime}(0)-120 f^{(4)}(0)+6160 f^{\prime}(0)^{4}+513 f^{\prime \prime}(0)^{2} \\
-\quad 672 g^{(3)}(0) f^{\prime}(0)-224 g^{\prime}(0) f^{(3)}(0)+234 g^{\prime \prime}(0) f^{\prime \prime}(0)-2136 g^{\prime}(0)^{3} f^{\prime}(0)+728 g^{\prime \prime}(0) f^{\prime}(0)^{2} \\
-\quad 448 g^{\prime}(0) g^{(3)}(0)-516 g^{\prime}(0)^{2} f^{\prime \prime}(0)+580 g^{\prime}(0)^{2} f^{\prime}(0)^{2}-3568 g^{\prime}(0) f^{\prime}(0)^{3}+2204 g^{\prime}(0)^{2} g^{\prime \prime}(0) \\
+\quad 2300 g^{\prime}(0) g^{\prime \prime}(0) f^{\prime}(0)+1884 g^{\prime}(0) f^{\prime \prime}(0) f^{\prime}(0)+120 g^{(4)}(0)-1036 g^{\prime}(0)^{4}-747 g^{\prime \prime}(0)^{2}, \\
q_{2}^{(4)}:=1344 f^{\prime}(0) f^{(3)}(0)-6600 f^{\prime}(0)^{2} f^{\prime \prime}(0)-120 f^{(4)}(0)+6160 f^{\prime}(0)^{+} 513 f^{\prime \prime}(0)^{2} \\
-\quad 99 g^{\prime \prime}(0)^{2}+84 g^{\prime}(0)^{4}+24 g^{(4)}(0)+64 g^{\prime}(0) g^{(3)}(0)-684 g^{\prime}(0)^{2} f^{\prime \prime}(0)+126 g^{\prime \prime}(0) f^{\prime \prime}(0) \\
-\quad 5712 f^{\prime}(0) f^{\prime}(0)^{3}+2436 g^{\prime}(0)^{2} f^{\prime}(0)^{2}-728 g^{\prime}(0)^{3} f^{\prime}(0)+712 g^{\prime \prime}(0) f^{\prime}(0)^{2}+92 g^{\prime}(0)^{2} g^{\prime \prime}(0) \\
-\quad 352 g^{\prime}(0) f^{(3)}(0)-288 g^{(3)}(0) f^{\prime}(0)+3156 g^{\prime}(0) f^{\prime \prime}(0) f^{\prime}(0)-36 g^{\prime}(0) g^{\prime \prime}(0) f^{\prime}(0) .
\end{gathered}
$$

a) $\operatorname{Se} q_{1}^{(2)} q_{2}^{(2)}>0$, então a origem é estável;

b) $S e q_{1}^{(2)} q_{2}^{(2)}<0$ ou somente um dos dois é nulo, então a origem é instável;

c) $S e q_{1}^{(2)}$ e $q_{2}^{(2)}$ são simultaneamente nulos e $q_{1}^{(4)} q_{2}^{(4)}>0$, então a origem é estável; se $q_{1}^{(4)} q_{2}^{(4)}<0$ ou somente um dos dois é nulo, então a origem é instável.

Demonstração. Os itens $a$ ) e b) estão demonstrados no Capítulo 4.

Vamos demonstrar o item $c$ ).

Para os cálculos envolvendo as derivadas das função $\tau, t_{1}$ e $t_{2}$ em zero, utilizaremos as fórmulas dadas no Teorema 5.2.1.

Da definição de $\bar{l}\left(x_{0}\right)$, temos que

$$
\lim _{x_{0} \rightarrow 0} \bar{l}\left(x_{0}\right)=\lim _{x_{0} \rightarrow 0} \frac{\tau\left(x_{0}\right)-t_{1}\left(x_{0}\right)}{\tau\left(x_{0}\right)-t_{2}\left(x_{0}\right)} .
$$


Como $\tau(0)=t_{1}(0)=t_{2}(0)=2 \pi$, obtemos uma indeterminação do tipo $0 / 0$. Usando a Regra de L'Hôpital:

$$
\lim _{x_{0} \rightarrow 0} \bar{l}\left(x_{0}\right)=\frac{\tau^{(1)}(0)-t_{1}^{(1)}(0)}{\tau^{(1)}(0)-t_{2}^{(1)}(0)} .
$$

Como $\tau^{\prime}(0)=t_{1}^{\prime}(0)=t_{2}^{\prime}(0)=0$, aplicamos novamente a regra de L'Hôpital

$$
\lim _{x_{0} \rightarrow 0} \bar{l}\left(x_{0}\right)=\frac{\tau^{(2)}(0)-t_{1}^{(2)}(0)}{\tau^{(2)}(0)-t_{2}^{(2)}(0)} .
$$

Temos que $\tau^{(2)}(0)-t_{1}^{(2)}(0)=\pi q_{1}^{(2)} / 12$ e $\tau^{(2)}(0)-t_{2}^{(2)}(0)=\pi q_{2}^{(2)} / 12$, assim

$$
\lim _{x_{0} \rightarrow 0} \bar{l}\left(x_{0}\right)=\frac{q_{1}^{(2)}}{q_{2}^{(2)}} .
$$

Como, por hipótese, $q_{1}^{(2)}=q_{2}^{(2)}=0$, utilizamos a regra de L'Hôpital

$$
\lim _{x_{0} \rightarrow 0} \bar{l}\left(x_{0}\right)=\frac{\tau^{(3)}(0)-t_{1}^{(3)}(0)}{\tau^{(3)}(0)-t_{2}^{(3)}(0)} .
$$

Fazendo $\tau^{(3)}(0)-t_{1}^{(3)}(0)$, reorganizando convenientemente os termos da expressão resultante e usando que $q_{1}^{(2)}(0)=0$, segue que $\tau^{(3)}(0)-t_{1}^{(3)}(0)=0$. De maneira análoga, usando que $q_{1}^{(2)}(0)=0$, obtemos $\tau^{(3)}(0)-t_{1}^{(3)}(0)=0$.

Da regra de L'Hôpital,

$$
\lim _{x_{0} \rightarrow 0} \bar{l}\left(x_{0}\right)=\frac{\tau^{(4)}(0)-t_{1}^{(4)}(0)}{\tau^{(4)}(0)-t_{2}^{(4)}(0)} .
$$

$\operatorname{Temos} \tau^{(4)}(0)-t_{1}^{(4)}(0)=\pi q_{1}^{(4)} / 192$ e $\tau^{(4)}(0)-t_{2}^{(4)}(0)=\pi q_{2}^{(4)} / 192$, logo

$$
\lim _{x_{0} \rightarrow 0} \bar{l}\left(x_{0}\right)=\frac{q_{1}^{(4)}}{q_{2}^{(4)}} .
$$

O restante da demonstração é análoga as dos itens $a$ ) e $b$ ).

A seguir, apresentamos algumas aplicações desse teorema.

Aplicação 7.3.1. Critério de estabilidade ou instabilidade para o origem do sistema:

$$
\left\{\begin{array}{l}
\ddot{x}=-x, \\
\ddot{y}=y g(x),
\end{array}\right.
$$

com g de classe $C^{4}$ e $g(0)=1$ :

a) se $g^{\prime}(0) \neq 0$ e

$$
-\frac{2}{3} \leq \frac{g^{\prime \prime}(0)}{g^{\prime}(0)^{2}} \leq \frac{10}{9}
$$

a origem é instável;

b) se $3 g^{\prime \prime}(0)<-2 g^{\prime}(0)^{2}$ ou $9 g^{\prime \prime}(0)>10 g^{\prime}(0)^{2}$, a origem é estável; 
c) se $g^{\prime}(0)=0, g^{\prime \prime}(0)=0$ e $g^{(4)}(0) \neq 0$, a origem é estável.

Exemplo 7.3.1. Se $g(x)=1+a x+b x^{2}+c x^{3}+d x^{4}$, com $a, b, c, d \in \mathbb{R}$, as condições são:

a) se $a \neq 0$ e

$$
-\frac{1}{3} \leq \frac{2 b^{2}}{a^{2}} \leq \frac{10}{9}
$$

a origem é instável;

b) se $c<-a^{2} / 3$ ou $c>5 a^{2} / 9$, a origem é estável;

c) se $a=0, b=0$ e $d \neq 0$, a origem é estável.

Na aplicação a seguir, consideramos $f$ e $g$ polinômios contendo apenas graus pares até o quarto grau e obtemos condições sobre seus coeficientes para que ocorra estabilidade ou instabilidade da origem.

Aplicação 7.3.2. Considere o sistema

$$
\left\{\begin{array}{l}
\dot{x}=-x\left(1+a x^{2}+b x^{4}\right) \\
\dot{y}=-y\left(1+c x^{2}+d x^{4}\right)
\end{array}\right.
$$

com $a, b, c, d \in \mathbb{R}:$

a) se $|c|<|a|$ ou $|c|>3|a|$, então a origem é estável;

b) se $|a| \leq|c|<3|a|$ ou $|a|<|c| \leq 3|a|$ a origem é instável;

c) se $a=c=0$ e $|d|<|b|$ ou $|d|>5|b|$, a origem é estável;

d) se $a=c=0$ e $|b| \leq|d|<5|b|$ ou $|b|<|d| \leq 5|b|$, então a origem é instável.

Exemplo 7.3.2. Considere os sistemas

$$
\left\{\begin{array} { l } 
{ \ddot { x } = - x ( 1 + x ^ { 2 } ) } \\
{ \ddot { y } = - y ( 1 + x ^ { 2 } - x ^ { 4 } ) }
\end{array} \quad \left\{\begin{array}{l}
\ddot{x}=-x\left(1+48 x^{4}\right) \\
\ddot{y}=-y\left(1+48 x^{4}\right)
\end{array}\right.\right.
$$

Em ambos sistemas a origem é instável. A instabilidade segue, respectivamente, dos itens b) $e$ d) da Aplicação 7.3.2.

Definição 7.3.1. Dizemos que o sistema é n-decidivel se a estabilidade ou instabilidade da origem pode ser decidida pelos valores das derivadas de $f(x)$ e $g(x)$ em $x=0$ de ordens 0 até $n$.

No exemplo acima, o primeiro sistema 2-decidível e o segundo é 4-decidível.

Supondo que $f, g$ são funções de classe $C^{\infty}$, com $g(0)=f(0)=1$. Definimos as quantidades

$$
\begin{aligned}
& s_{1}^{(k)}=\tau^{(k)}(0)-t_{1}^{(k)}(0) \\
& s_{2}^{(k)}=\tau^{(k)}(0)-t_{2}^{(k)}(0) .
\end{aligned}
$$

Motivados pela demonstração do Teorema 7.3.2 e pela teoria desenvolvida nos capítulos anteriores, enunciamos o resultado a seguir, que deixa claro que $\tau\left(x_{0}\right)$-isocronismo é uma situação de estabilidade que não e finitamente decidível, que é um fato de todo esperado. 
Teorema 7.3.3. Seja (5.1) com $f$ e $g$ positivas numa vizinhança da origem, de classe $C^{\infty} e$ $g(0)=f(0)=1$ :

i)- se o sistema possui $\tau\left(x_{0}\right)$-isocronismo, então

$$
s_{1}^{(n)}=s_{2}^{(n)}=0, \quad \forall n \in \mathbb{N} .
$$

Neste caso, a origem do sistema é estável. Mas não existe natural n tal que o sistema seja $n$-decidivel.

ii)- se existe $\bar{k} \in \mathbb{N}$ tal que, para todo $k$ natural, com $k<\bar{k}$ :

$$
s_{1}^{(k)}=s_{2}^{(k)}=0 \quad e \quad s_{1}^{(\bar{k})} s_{2}^{(\bar{k})}>0,
$$

então a origem do sistema é estável (não inclui o caso em que ocorre $\tau\left(x_{0}\right)$-isocronismo) e o sistema é $\bar{k}$ - decidível;

iii)- se existe $\bar{k} \in \mathbb{N}$ tal que, para todo $k$ natural, com $k<\bar{k}$ :

$$
s_{1}^{(k)}=s_{2}^{(k)}=0 \quad e \quad s_{1}^{(\bar{k})} s_{2}^{(\bar{k})}<0,
$$

ou somente um dos dois é nulo, então a origem do sistema é instável e o sistema é $\bar{k}$-decidível.

Demonstração. O item $i$ ) segue do Teorema 5.1 .2 e as demonstrações dos itens ii) e iii) é similar à demonstração do Teorema 7.3.2.

Observamos que versões do teorema acima são válidas quando $f$ e $g$ são funções de classe $C^{r}$ ou analíticas. No caso de $f$ e $g$ analíticas, no item $i$ ), temos uma condição necessária e suficiente para ocorrência do $\tau\left(x_{0}\right)$-isocronismo. 


\section{Capítulo 8}

\section{Considerações finais}

Nesta tese estudamos sistemas de forças quase-centrais tais que as funções $f$ e $g$ dependem apenas da variável $x$, isto é,

$$
\ddot{x}=-x f(x), \quad \ddot{y}=-y g(x), \quad f, g \in C^{\omega} .
$$

Nosso problema consiste em: fixada $f$, procurar condições sobre $g(x)$ para existir $\delta>0$ tal que, as soluções do sistema com condições inicias $x(0)=x_{0}, \dot{x}=0, y(0)=y_{0}, \dot{y}(0)=\dot{y}_{0}$ sejam periódicas de menor período $\tau\left(x_{0}\right), \forall\left(x_{0}, y_{0}, \dot{y}_{0}\right) \neq 0$ com $0 \leq x_{0}<\delta$, em que $\tau\left(x_{0}\right)$ é o menor período da solução da primeira equação do sistema com condições iniciais $x(0)=x_{0}, \dot{x}(0)=0$. Denotamos esse fenômeno por $\tau\left(x_{0}\right)$-isocronismo.

Esse problema pode ser abordado em diferentes aspectos. Como um problema de isocronismo de "sistema mecânico", linha abordada por G.Zampieri em [Zam11], ou na linha de coexistência de soluções periódicas abordada pelo mesmo em [Zam89]. Além disso, podemos encará-lo também sob aspecto de equações de Hill, ou seja, obter condições sob uma família de equações de Hill que possuam uma propriedade de isocronismo extremamente forte, o $\tau\left(x_{0}\right)$-isocronismo.

Nosso resultado principal estabelece que para $f$ analítica real, definida e positiva numa vizinhança da origem, $I$, com $f(0)=1$ e satisfazendo $3 f^{\prime \prime}(0)>4 f^{\prime}(0)^{2}$, existem no máximo duas funções $g$, analíticas reais, definidas e positivas em $I$, tal que o sistema possui $\tau\left(x_{0}\right)$-isocronismo. Além disso, exibimos como será o resultado em série de potências das $g$ com essa propriedade, isso permite determinar explicitamente os possíveis jatos de ordem $k$ de $g(x)$ em 0 . Esses ficam completamente determinados por $j^{(k)} g(0)$ e $j^{(k+1)} f(0)$.

Quando $3 f^{\prime \prime}(0)=4 f^{\prime}(0)^{2}$ não se tem o mesmo tipo de resultado, pois o sistema é degenerado. Neste caso, conseguimos determinar apenas os jatos pares de $g$ em 0 . O caso $f=g$ está contido nessa classe de sistemas.

Para $3 f^{\prime \prime}(0)<4 f^{\prime}(0)^{2}$ mostramos que não existe $g$ analítica real tal que o sistema possui $\tau\left(x_{0}\right)$ isocronismo.

Destacamos que isto não é surpreendente, do ponto de vista dos trabalhos de isocronismo, que existam tão poucas possibilidades para a $g$. Uma questão notável é a importância do jato 2 de $f$ em 0 para determinar questões de existência e degenerecência de $g$.

Outro fato notável é a importância que as funções "tempos de parada", $\left(t_{1}, t_{2}\right)$, têm neste problema, de fato, o Teorema 5.1.2 deixa isso evidente.

Os temas abordados nessa tese dão impulso à novas questões interessantes que serão abordadas 
em trabalhos futuros.

Uma delas é estudar as condições de convergência da série de potências das funções $g$ obtidas no nosso teorema, talvez a mais urgente, mas também uma das mais delicadas. A outra é analisar com mais detalhes o caso $3 f^{\prime \prime}(0)=4 f^{\prime}(0)^{2}$, caso em que o sistema é degenerado. 


\section{Apêndice A}

\section{Derivadas de $\tau, t_{1}$ e $t_{2}$ em zero até a quarta ordem}

Para o cálculo das derivadas de $\tau, t_{1}$ e $t_{2}$, utilizamos o software Maple. Aqui, apresentaremos parte das contas envolvidas que não estão presentes na Seção 5.2.1. As constantes $a, b, c, d, \alpha, \beta, \gamma, \omega$ denotam, respectivamente, $f^{\prime}(0), f^{\prime \prime}(0), f^{(3)}(0), f^{(4)}(0), g^{\prime}(0), g^{\prime \prime}(0), g^{(3)}(0), g^{(4)}(0)$.

\section{A.1 Algumas contas referentes ao cálculo das derivadas de $\tau$ em zero até a quarta ordem}

$$
\dot{\mu}_{2}(2 \pi)=0, \quad \ddot{\mu}_{2}(2 \pi)=-2 a, \quad \dot{\mu}_{3}(2 \pi)=5 a^{2} \pi-\frac{9}{4} b \pi, \quad \ddot{\mu}_{3}(2 \pi)=-3 b .
$$

A expressão de $\mu_{4}(t)$ é:

$$
\begin{aligned}
\mu_{4}(t) & =-5 / 2 c+105 / 8 a b+38 / 3 a^{3} \cos (t)^{2}+2 \cos (t)^{2} c+29 / 18 a^{3} \cos (t)-113 / 6 a^{3}+4 / 5 \cos (t) c \\
& -3 / 16 \cos (t) \cos (5 t) a b+25 / 16 \cos (t) a b \cos (3 t)-5 / 3 \cos (t) a^{3} t \sin (3 t)-3 / 2 \cos (t) a b \cos (2 t) \\
& -3 / 8 \cos (t) a b \cos (4 t)-3 / 16 \sin (t) \sin (5 t) a b+15 / 16 \sin (t) \sin (3 t) a b-3 \sin (t) \sin (2 t) a b \\
& +5 / 3 \sin (t) a^{3} t \cos (3 t)-3 / 8 \sin (t) \sin (4 t) a b-1 / 20 \sin (t) \sin (5 t) c-1 / 20 \cos (t) \cos (5 t) c \\
& -1 / 4 \cos (t) \cos (3 t) c+4 \sin (t) \sin (2 t) a^{3}-21 / 2 a b \cos (t)^{2}-31 / 36 a^{3} \cos (t) \cos (3 t) \\
& -1 / 2 \sin (t) \sin (4 t) a^{3}-1 / 12 \sin (t) \sin (5 t) a^{3}-9 / 2 a b t \sin (t)+3 / 4 \cos (t) a b t \sin (3 t) \\
& -3 / 4 \sin (t) a b t \cos (3 t)-9 / 2 a \cos (t) b t \sin (t)-1 / 12 \cos (t) \cos (5 t) a^{3}-1 / 2 \cos (t) a^{3} \cos (4 t) \\
& -41 / 36 \sin (t) \sin (3 t) a^{3}-5 / 12 \sin (t) \sin (3 t) c+10 a^{3} t \sin (t)+6 a^{3} \cos (t) \cos (2 t) \\
& -17 / 8 a b \cos (t)+10 a^{3} \cos (t) t \sin (t) .
\end{aligned}
$$

Derivando com respeito à $t$ e calculando em $t=2 \pi$,

$$
\dot{\mu}(2 \pi)=-15 a b \pi+\frac{100}{3} a^{3} \pi
$$


A expressão de $\mu_{5}(t)$ é obtida resolvendo o sistema

$$
\begin{gathered}
\ddot{\mu_{5}}(t)+\mu_{5}(t)=-5 a \mu_{1}(t) \mu_{4}(t)-10 \mu_{3}(t)\left[b\left(\mu_{1}(x)\right)^{2}+a \mu_{2}(t)\right]-10 \mu_{2}(t)\left[c\left(\mu_{1}(t)\right)^{3}+3 b \mu_{2}(t) \mu_{1}(t)+\mu_{3}(t) a\right] \\
-5 \mu_{1}(t)\left[d\left(\mu_{1}(t)\right)^{4}+6 c \mu_{2}(t) \mu_{1}(t)^{2}+4 \mu_{3}(t) \mu_{1}(t) b+3 b\left(\mu_{2}(t)\right)^{2}+\mu_{4}(t) a\right] \\
\mu_{5}(0)=0, \quad \dot{\mu}_{5}(0)=0 .
\end{gathered}
$$

$$
\begin{aligned}
\mu_{5}(t) & =760 / 9 a^{4} \cos (t)^{2}-375 / 512 \cos (t) b^{2}+55 / 96 \cos (t) d-30 a^{2} \cos (t) b t \sin (t)+185 / 288 a^{4} \cos (t) \\
& +5 \cos (t) a^{2} b t \sin (3 t)-15 / 16 \cos (t) a^{2} b t \sin (4 t)-15 / 2 \cos (t) a^{2} b t \sin (2 t)-5 \sin (t) a^{2} b t \cos (3 t) \\
& +15 / 6 \sin (t) a^{2} b t \cos (4 t)+235 / 2 a^{2} b-135 / 32 \sin (t) b^{2} t \cos (2 t)-31 / 9 \sin (t) \sin (3 t) a c \\
& -1 / 3 \cos (t) \cos (5 t) a c-5 / 4 \sin (t) \sin (5 t) a^{2} b+75 / 8 \cos (t) a^{2} b t^{2}+50 / 3 \cos (t) a^{4} t \sin (2 t) \\
& -7 / 3 \cos (t) \cos (3 t) a c+55 / 4 \cos (t) \cos (3 t) a^{2} * b-5 / 4 \cos (t) \cos (5 t) a^{2} b-11 / 72 \cos (t) \cos (6 t) a c \\
& -25 / 96 \cos (t) \cos (6 t) a^{2} b-25 / 8 \cos (t) a^{4} x \sin (4 t)+115 / 12 \sin (t) a^{2} b \sin (3 t) \\
& +1100 / 9 \sin (t) a^{4} t \cos (3 t)-25 / 96 \sin (t) \sin (6 t) a^{2} b-1 / 3 \sin (t) \sin (5 t) a c-11 / 72 \sin (t) \sin (6 t) a c \\
& +35 / 2 \sin (t) a c t+5 / 12 \cos (t) \cos (4 t) a c+135 / 128 \cos (t) b^{2} t \sin (4 t)+165 / 64 \cos (t) \cos (4 t) a^{2} b \\
& -100 / 9 \cos (t) a^{4} t \sin (3 t)+67 / 8 \cos (t) \cos (2 t) a c+73 / 8 \sin (t) \sin (2 t) a c-1 / 24 \sin (t) \sin (4 t) a c \\
& -135 / 128 \sin (t) b^{2} t \cos (4 t)+115 / 64 \sin (t) a^{2} b \sin (4 t)+25 / 8 \sin (t) a^{4} t \cos (4 t) \\
& -1115 / 32 b \sin (2 t) \sin (t) a^{2}-1365 / 32 \cos (t) \cos (2 t) a^{2} b-25 / 6 a^{4} t \sin (t) \cos (2 t) \\
& +200 / 3 a^{4} \cos (t) t \sin (t)+135 / 512 \cos (t) b^{2} \cos (4 t)+2525 / 36 a^{4} t \sin (t)-52 / 3 a \cos (t)^{2} c \\
& -1130 / 9 a^{4}+65 / 192 a^{2} b \cos (t)-23 / 36 \cos (t) a c-90 a^{2} \cos (t)^{2} b-1435 / 288 \sin (t) a^{4} \sin (4 t) \\
& -5 / 192 \sin (t) \sin (6 t) d-25 / 16 \sin (t) d t+4165 / 144 \sin (t) \sin (2 t) a^{4}+45 / 512 \sin (t) b^{2} \sin (4 t) \\
& -1385 / 288 \cos (t) \cos (4 t) a^{4}-15 / 256 \cos (t) \cos (6 t) b^{2}-25 / 432 \cos (t) \cos (6 t) a^{4} \\
+ & 7435 / 144 a^{4} \cos (t) \cos (2 t)-5 / 9 \sin (t) \sin (5 t) a^{4}-25 / 432 \sin (t) \sin (6 t) a^{4}-75 / 64 \sin (t) \sin (2 t) d \\
+ & 585 / 256 \sin (t) \sin (2 t) b^{2}-205 / 27 \sin (t) a^{4} \sin (3 t)-15 / 256 \sin (t) \sin (6 t) b^{2}-15 / 64 \sin (t) \sin (4 t) d \\
+ & 45 / 64 \sin (t) b^{2} t-125 / 12 \cos (t) a^{4} x^{2}-5 / 9 \cos (t) \cos (5 t) a^{4}-155 / 27 a^{4} \cos (3 t) \cos (t) \\
+ & 45 / 4 b t \sin (t) a^{2} \cos (2 t)-545 / 8 b t \sin (t) a^{2}-68 / 3 a c . \\
&
\end{aligned}
$$

Derivando com respeito à $t$ e calculando em $t=2 \pi$,

$$
\dot{\mu}_{5}(2 \pi)=35 a c \pi-\frac{1215}{8} b a^{2} \pi-\frac{25}{8} d \pi+\frac{2725}{12} a^{4} \pi-\frac{585}{64} b^{2} \pi .
$$

\section{A.2 Algumas contas referentes ao cálculo das derivadas de $t_{1} \mathrm{em}$ zero até a quarta ordem}

$$
\dot{v}_{1}(2 \pi)=0, \quad \ddot{v}_{1}(2 \pi)=-\alpha, \quad \dot{v}_{2}(2 \pi)=\frac{5}{6} a \alpha \pi+\frac{5}{6} \alpha^{2} \pi-\frac{3}{4} \beta \pi, \quad \ddot{v}_{2}(2 \pi)=-\beta
$$


A expressão de $v_{3}(t)$ é obtida resolvendo o sistema:

$$
\begin{gathered}
\ddot{v}_{3}(t)+v_{3}(t)=-3 \alpha \mu_{1}(t) v_{2}(t)-3 \beta v_{1}(t) \mu_{1}(t)^{2}-3 \alpha v_{1}(t) \mu_{2}(t) \\
-\gamma v_{0}(t) \mu_{1}(t)^{3}-3 \beta v_{0}(t) \mu_{2}(t) \mu_{1}(t)-\alpha v_{0}(t) \mu_{3}(t), \\
v_{3}(0)=0, \quad \dot{v}_{3}(0)=0 .
\end{gathered}
$$

$v_{3}(t)=-5 / 8 \gamma+3 / 32 \cos (t) \alpha \beta t \sin (3 t)-25 / 32 \alpha^{3}-227 / 96 \alpha^{2} a+77 / 64 \alpha \beta+23 / 24 \alpha^{3} \cos (t)^{2}$

$-229 / 720 \alpha^{3} \cos (t)+1 / 2 \cos (t)^{2} \gamma+7 / 4 a \beta-25 / 16 \alpha a^{2}+21 / 64 \alpha b-3 / 4 \sin (t) \beta a t$

$-1 / 4 \sin (t) \sin (2 t) \alpha \beta+1 / 2 \sin (t) \sin (2 t) \alpha^{2} a-1 / 16 \cos (t) \cos (4 t) \alpha^{2} a-1 / 16 \cos (t) \cos (4 t) \beta a$

- $11 / 960 \sin (t) \sin (5 t) \alpha^{2} a-1 / 32 \cos (t) \cos (4 t) \alpha \beta-1 / 24 \cos (x) \cos (4 x) \alpha a^{2}+977 / 720 \alpha^{2} \cos (x) a$

- $229 / 360 \alpha \cos (t) a^{2}+1 / 6 \sin (t) \sin (2 t) \alpha^{3}-5 / 48 \sin (t) \sin (3 t) \gamma-1 / 48 \cos (t) \cos (4 t) \alpha^{3}$

$+5 / 12 \alpha^{3} t \sin (t)-1 / 320 \sin (t) \sin (5 t) \alpha^{3}-1 / 16 \cos (t) \cos (3 t) \gamma-1 / 48 \sin (t) \sin (4 t) \alpha^{3}$

- $1 / 80 \sin (t) \sin (5 t) \gamma-53 / 480 \alpha \beta \cos (t)-1 / 320 \cos (t) \cos (5 t) \alpha^{3}-49 / 576 \cos (t) \alpha^{3} \cos (3 t)$

$+1 / 4 \cos (t) \cos (2 t) \alpha^{3}+7 / 24 \alpha^{2} \cos (t)^{2} a+23 / 12 \alpha \cos (t)^{2} a^{2}-55 / 576 \sin (t) \sin (3 t) \alpha^{3}$

- $9 / 16 \alpha b \cos (t)^{2}-149 / 240 \beta a \cos (t)+1 / 5 \alpha b \cos (t)-1 / 80 \cos (t) \cos (5 t) \gamma-\beta \cos (t)^{2} a$

$+5 / 8 \alpha^{2} \cos (t) \sin (t) a t-1 / 4 \cos (t) \cos (2 t) \beta a-3 / 8 \alpha \beta t \sin (t)+5 / 128 \cos (t) \alpha b \cos (3 t)$

$-1 / 8 \cos (t) \cos (2 t) \alpha \beta+5 / 6 \alpha a^{2} t \sin (t)+5 / 4 \alpha^{2} a t \sin (t)+55 / 384 \cos (t) \alpha \beta \cos (3 t)$

- $1 / 160 \cos (t) \cos (5 t) \alpha a^{2}+5 / 24 \cos (t) \cos (3 t) \beta a-5 / 48 \cos (t) \alpha^{3} t \sin (3 t)+23 / 576 \cos (t) \alpha^{2} a \cos (3 t)$

$-1 / 2 \sin (t) \sin (2 t) \beta a-11 / 960 \cos (t) \cos (5 t) \alpha^{2} a-3 / 640 \cos (t) \cos (5 t) \alpha b-1 / 40 \cos (t) \cos (5 t) \beta a$

- $11 / 640 \cos (t) \cos (5 t) \alpha \beta+1 / 576 \sin (t) \sin (3 t) \alpha^{2} a-55 / 288 \sin (t) \sin (3 t) \alpha a^{2}+1 / 3 \sin (t) \sin (2 t) \alpha a^{2}$

- $1 / 16 \sin (t) \sin (4 t) \alpha^{2} a-1 / 16 \sin (t) \sin (4 t) \beta a+11 / 128 \sin (t) \sin (3 t) \alpha \beta+3 / 128 \sin (t) \sin (3 t) \alpha b$

$-9 / 16 \alpha \cos (t) b t \sin (t)+5 / 4 \alpha \cos (t) a^{2} t \sin (t)-9 / 16 \alpha \cos (t) \sin (t) \beta t+3 / 32 \cos (t) \alpha b t \sin (3 t)$

$+\quad 5 / 48 \sin (t) \alpha^{2} a t \cos (3 t)+5 / 24 \sin (t) \alpha a^{2} t \cos (3 t)-3 / 32 \sin (t) \alpha \beta t \cos (3 t)+1 / 5 \cos (t) \gamma$

$-3 / 32 \sin (t) \alpha b t \cos (3 t)+1 / 8 \sin (t) \sin (3 t) \beta a-3 / 640 \sin (t) \sin (5 t) \alpha b-1 / 40 \sin (t) \sin (5 t) \beta a$

- $11 / 640 \sin (t) \sin (5 t) \alpha \beta-1 / 160 \sin (t) \sin (5 t) \alpha a^{2}+5 / 48 \sin (t) \alpha^{3} t \cos (3 t)-1 / 32 \sin (t) \sin (4 t) \alpha \beta$

$-1 / 24 \sin (t) \sin (4 t) \alpha a^{2}+5 / 8 \alpha^{3} \cos (t) \sin (t) t+3 / 4 \alpha^{2} a \cos (t) \cos (2 t)+1 / 2 \alpha \cos (t) a^{2} \cos (2 t)$

- $49 / 288 \alpha \cos (t) a^{2} \cos (3 t)-17 / 16 \alpha \cos (t)^{2} \beta-5 / 24 \cos (t) \alpha a^{2} t \sin (3 t)-5 / 48 \cos (t) \alpha^{2} a t \sin (3 t)$.

Derivando com respeito à $t$ e calculando em $t=2 \pi$

$$
\dot{v}_{3}(2 \pi)=\frac{5}{3} \alpha^{3} \pi+\frac{10}{3} \alpha a^{2} \pi-\frac{3}{2} \alpha \beta \pi-\frac{3}{2} \beta a \pi-\frac{3}{4} \alpha b \pi+\frac{10}{3} \alpha^{2} a \pi .
$$

A expressão de $v_{4}(t)$ é obtida resolvendo o sistema:

$$
\begin{gathered}
\ddot{v}_{4}(t)+v_{4}(t)=-4 \alpha \mu_{1}(t) v_{3}(t)+6 v_{2}(t)\left[-\beta\left(\mu_{1}(t)\right)^{2}-\alpha \mu_{2}(t)\right] \\
+4 v_{1}(t)\left[-\gamma\left(\mu_{1}(t)\right)^{3}-3 \mu_{2}(t) \mu_{1}(t) \beta-\mu_{3}(t) \alpha\right] \\
+\quad v_{0}(t)\left[-\omega\left(\mu_{1}(t)\right)^{4}-6 \gamma \mu_{2}(t) \mu_{1}(t)^{2}-4 \beta \mu_{3}(t) \mu_{1}(t)-3 \beta\left(\mu_{2}(t)\right)^{2}-\mu_{4}(t) \alpha\right], \\
v_{4}(0)=0, \quad \dot{v}_{4}(0)=0 .
\end{gathered}
$$


Derivando $v_{4}(t)$ com respeito à $t$ e calculando em $t=2 \pi$,

$$
\begin{aligned}
\dot{v}_{4}(2 \pi) & =-\frac{5}{16} \alpha^{2} b \pi-\frac{91}{24} \beta a^{2} \pi+\frac{7}{6} \alpha c \pi-\frac{157}{16} \alpha b a \pi+\frac{165}{16} \alpha^{2} a^{2} \pi+\frac{33}{16} \alpha^{4} \pi-\frac{39}{32} \beta b \pi+\frac{7}{2} \gamma a \pi-\frac{623}{48} \beta \alpha a \pi \\
& +\frac{223}{12} \alpha a^{3} \pi-\frac{167}{48} \alpha^{2} \beta p i+\frac{7}{3} \alpha \gamma \pi+\frac{347}{24} \alpha^{3} a \pi-\frac{5}{8} \omega \pi-\frac{39}{64} \beta^{2} \pi .
\end{aligned}
$$

\section{A.3 Algumas contas referentes ao cálculo das derivadas de $t_{2} \mathrm{em}$ zero até a quarta ordem}

$$
\dot{u}_{1}(2 \pi)=0, \quad u_{2}(2 \pi)=-\frac{7}{6} \alpha a \pi+\frac{1}{4} \beta \pi+\frac{1}{6} \alpha^{2} \pi, \quad \dot{u}_{2}(2 \pi)=0
$$

A expressão de $u_{3}(t)$ é obtida resolvendo o sistema:

$$
\begin{gathered}
\ddot{u}_{3}(t)+u_{3}(t)=-3 \alpha \mu_{1}(t) u_{2}(t)-3 \beta u_{1}(t) \mu_{1}(t)^{2}-3 \alpha u_{1}(t) \mu_{2}(t) \\
-\quad u_{0}(t) \gamma \mu_{1}(t)^{3}-3 \beta u_{0}(t) \mu_{2}(t) \mu_{1}(t)-\alpha u_{0}(t) \mu_{3}(t), \\
u_{3}(0)=0, \quad \dot{u}_{3}(0)=0 .
\end{gathered}
$$

$u_{3}(t)=-1 / 5 \sin (t) \gamma+139 / 720 \alpha^{3} \sin (t)+7 / 48 \sin (t) \alpha^{2} a t \sin (3 t)+7 / 48 \cos (t) \alpha^{2} a t \cos (3 t)$

$+1 / 16 \sin (t) \cos (3 t) \gamma-1 / 12 \sin (t) \cos (2 t) \alpha^{3}-1 / 48 \sin (t) \cos (4 t) \alpha^{3}+1 / 320 \sin (t) \cos (5 t) \alpha^{3}$

$+1 / 48 \cos (t) \sin (4 t) \alpha^{3}+9 / 32 \alpha b t-5 / 8 \alpha a^{2} t-9 / 32 \alpha \beta t-1147 / 720 \alpha^{2} a \sin (t)-7 / 8 \alpha^{2} \cos (t)^{2} a t$

$+3 / 16 \alpha \cos (t)^{2} \beta t-7 / 32 \alpha \cos (t) \beta \sin (t)+5 / 12 \alpha^{2} a \sin (t) \cos (2 t)+43 / 24 \alpha \sin (t) a^{2} \cos (t)$

- $1 / 2 \alpha \sin (t) a^{2} \cos (2 t)-3 / 2 \beta \sin (t) a \cos (t)+49 / 288 \alpha \sin (t) a^{2} \cos (3 t)+25 / 48 \alpha^{2} \cos (t) a \sin (t)$

- $1 / 48 \sin (t) \alpha^{3} t \sin (3 t)+5 / 576 \sin (t) \alpha^{2} a \cos (3 t)-5 / 24 \sin (t) \cos (3 t) \beta a+1 / 4 \sin (t) \cos (2 t) \beta a$

$+11 / 960 \sin (t) \cos (5 t) \alpha^{2} a+5 / 384 \sin (t) \alpha \beta \cos (3 t)+1 / 160 \sin (x) \cos (5 t) \alpha a^{2}-1 / 8 \sin (t) \cos (2 t) \alpha \beta$

$+1 / 48 \sin (t) \cos (4 t) \alpha^{2} a+1 / 24 \sin (t) \cos (4 t) \alpha a^{2}-1 / 32 \sin (t) \cos (4 t) \alpha \beta+1 / 16 \sin (t) \cos (4 t) \beta a$

$+11 / 640 \sin (t) \cos (5 t) \alpha \beta+1 / 40 \sin (t) \cos (5 t) \beta a-5 / 128 \sin (t) \alpha b \cos (3 t)-21 / 32 \sin (t) \cos (t) \alpha b$

$+7 / 24 \cos (t) \sin (3 t) \beta a+17 / 384 \cos (t) \sin (3 t) \alpha \beta+2 / 3 \cos (t) \sin (2 t) \alpha a^{2}-1 / 3 \cos (t) \sin (2 t) \alpha^{2} a$

- $1 / 48 \cos (t) \alpha^{3} t \cos (3 t)+1 / 32 \cos (t) \sin (4 t) \alpha \beta-5 / 4 \alpha a^{2} t \cos (t)^{2}-3 / 32 \sin (t) \alpha b t \sin (3 t)$

$+5 / 24 \sin (t) \alpha a^{2} t \sin (3 t)-1 / 32 \sin (t) \alpha \beta t \sin (3 t)+5 / 24 \cos (t) \alpha a^{2} t \cos (3 a t)-3 / 16 \alpha^{3} t$

- $1 / 32 \cos (t) \alpha \beta t \cos (3 t)-3 / 32 \cos (t) \alpha b t \cos (3 t)+1 / 4 \cos (t) \beta a t-1 / 24 \cos (t) \sin (4 t) \alpha a^{2}$

- $1 / 48 \cos (t) \sin (4 t) \alpha^{2} a+9 / 16 \alpha b t \cos (t)^{2}+3 / 640 \sin (t) \cos (5 t) \alpha b-43 / 288 \cos (t) \sin (3 t) \alpha a^{2}$

$+\quad 7 / 128 \cos (t) \sin (3 t) \alpha b+17 / 576 \cos (t) \sin (3 t) \alpha^{2} a-1 / 160 \cos (t) \sin (5 t) \alpha^{2}+1 / 8 \alpha^{3} \cos (t)^{2} t$

- $1 / 40 \cos (t) \sin (5 t) \beta a-11 / 640 \cos (t) \sin (5 t) \alpha \beta-1 / 16 \cos (t) \sin (4 t) \beta a+1 / 48 \alpha^{3} \cos (t) \sin (t)$

$+11 / 960 \cos (t) \sin (5 t) \alpha^{2} a-3 / 640 \cos (t) \sin (5 t) \alpha b+3 / 4 \alpha^{2} a t \cos (t)-7 / 6 \alpha a^{2} t \cos (t)$

$-1 / 8 \alpha \beta t \cos (t)+1 / 8 \sin (t) \cos (5 t) \gamma+149 / 240 \sin (t) \beta a+1 / 4 \sin (t) \cos (t) \gamma-1 / 12 \alpha^{3} t \cos (t)$

- $1 / 320 \cos (t) \sin (5 t) \alpha^{3}-1 / 48 \cos (t) \sin (3 t) \gamma+13 / 576 \sin (t) \alpha^{3} \cos (3 t)-1 / 12 \alpha^{3} t \cos (t)$

- $7 / 576 \cos (t) \sin (3 t) \alpha^{3}-1 / 5 \alpha b \sin (t)+203 / 480 \alpha \beta \sin (t)-1 / 80 \cos (t) \sin (5 t) \gamma+21 / 16 \alpha^{2} a t$

$+229 / 360 \alpha \sin (t) a^{2}$. 
Calculando em $t=2 \pi$

$$
u_{3}(2 \pi)=-\frac{17}{3} \alpha a^{2} \pi+\frac{8}{3} \alpha^{2} a \pi-\frac{1}{3} \alpha^{3} \pi-\frac{1}{2} \alpha \beta \pi+\frac{3}{2} \alpha b \pi+\frac{1}{2} \beta a \pi .
$$

A expressão de $u_{4}(t)$ é obtida resolvendo o sistema:

$$
\begin{gathered}
\ddot{u}_{4}(t)+u_{4}(t)=-4 \alpha \mu_{1}(t) u_{3}(t)+6 u_{2}(t)\left[-\beta\left(\mu_{1}(t)\right)^{2}-\alpha \mu_{2}(t)\right] \\
+4 u_{1}(t)\left[-\gamma\left(\mu_{1}(t)\right)^{3}-3 \mu_{2}(t) \mu_{1}(t) \beta-\mu_{3}(t) \alpha\right] \\
+u_{0}(t)\left[-h\left(\mu_{1}(t)\right)^{4}-6 \gamma \mu_{2}(t) \mu_{1}(t)^{2}-4 \beta \mu_{3}(t) \mu_{1}(t)-3 \beta\left(\mu_{2}(t)\right)^{2}-\mu_{4}(t) \alpha\right], \\
u_{4}(0)=0, \quad \dot{u}_{4}(0)=0 .
\end{gathered}
$$

Calculando em $t=2 \pi$

$$
\begin{aligned}
u_{4}(2 \pi) & =-\frac{33}{64} \beta^{2} \pi+\frac{7}{16} \alpha^{4} \pi+\frac{1}{8} \omega \pi+\frac{1}{3} \alpha \gamma \pi-\frac{57}{16} \alpha^{2} b \pi+\frac{21}{32} \beta b \pi-\frac{119}{4} \alpha a^{3} \pi \\
& +\frac{203}{16} \alpha^{2} a^{2} \pi-\frac{91}{24} \alpha^{3} a \pi+\frac{89}{24} \beta a^{2} \pi+\frac{23}{48} \alpha^{2} \beta \pi-\frac{263}{16} \alpha b a \pi-\frac{3}{16} \alpha \beta a .
\end{aligned}
$$


APÊNDICE A 


\section{Apêndice B}

\section{Demonstrações dos lemas auxiliares}

\section{B.1 Demonstração do Lema 6.1.3}

Lema B.1.1. Para todo q natural, vale

$$
A_{1}^{2 q}=-\frac{4}{3} a_{2 q} \pi(2 q+3)\left(\frac{(2 q-1) ! !}{(2 q+2) ! !}\right)
$$

Demonstração. Devemos resolver o sistema:

$$
\begin{gathered}
\ddot{v}_{2 q}+v_{2 q}=-a_{2 q} v_{0}(t) \mu_{1}(t)^{2 q-2}(t) \mu_{2}(t) g^{(2 q-1)(0)} \\
v_{2 q}(0)=0, \quad \dot{v}_{2 q}(0)=0
\end{gathered}
$$

derivar a solução com respeito a $t$ e calcular em $t=2 \pi$.

Substituindo $v_{0}(t), \mu_{1}(t)$ e $\mu_{2}(t)$, temos

$$
\begin{gathered}
\ddot{v}_{2 q}+v_{2 q}=-a_{2 q} \cos (t)^{(2 q-1)}\left(\frac{2}{3} \cos (t)+\frac{1}{3} \cos (2 t)-1\right) \\
v_{2 q}(0)=0, \quad \dot{v}_{2 q}(0)=0,
\end{gathered}
$$

Reescrevemos (B.2) em sistemas independentes e depois encontramos as soluções desses sistema. A soma destas soluções é a solução geral do sistema original, ou seja, $v_{2 q}(t)$.

- Resolução do sistema $(S 1)$

$$
\begin{gathered}
\ddot{v}_{2 q}^{(1)}+v_{2 q}^{(1)}=-\frac{2}{3} a_{2 q} \cos (t)^{2 q} \\
v_{2 q}^{(1)}(0)=0, \quad \dot{v}_{2 q}^{(1)}(0)=0,
\end{gathered}
$$

Seja $K_{1}=\frac{2}{3} a_{2 q}$.

Aplicando o Método da Variação dos Parâmetros a solução do sistema é

$$
\begin{aligned}
v_{2 q}^{(1)}(t) & =K_{1}\left[\left(\int \sin (t)(\cos (t))^{2 q} d t\right) \cos (t)-\left(\int(\cos (t))^{2 q+1} d t\right) \sin (t)\right. \\
& \left.+c_{1} \cos (t)+c_{2} \sin (t)\right],
\end{aligned}
$$


Como, $\forall q \in \mathbb{N}$

$$
\int \sin (t)(\cos (t))^{2 q} d t=-\frac{1}{2 q+1} \cos (t)^{(2 q+1)}
$$

$$
\int(\cos (t))^{2 q+1} d t=\frac{(2 q) ! !}{(2 q+1) ! !}\left(\sin (t) \sum_{p=0}^{q} \frac{(2 p-1) ! !}{(2 p) ! !}(\cos (t))^{2 p}\right),
$$

então

$$
\begin{aligned}
v_{2 q}^{(1)}(t) & =K_{1}\left(-\frac{1}{2 q+1}(\cos (t))^{2 q+2}+\frac{1}{2 q+1} \cos (t)\right) \\
& -K_{1} \frac{(2 q) ! !}{(2 q+1) ! !}\left((\sin (t))^{2} \sum_{p=0}^{q} \frac{(2 p-1) ! !}{(2 p) ! !}(\cos (t))^{2 p}\right)
\end{aligned}
$$

- Resolução do sistema $(S 2)$

$$
\begin{gathered}
\ddot{v}_{2 q}^{(2)}+v_{2 q}^{(2)}=-\left(-a_{2 q} \cos (t)^{2 q-1}\right) \\
v_{2 q}^{(2)}(0)=0, \quad \dot{v}_{2 q}^{(2)}(0)=0
\end{gathered}
$$

Seja $k_{2}=-a_{2 q}$.

Aplicando o Método da Variação dos Parâmetros a solução do sistema é

$$
\begin{aligned}
v_{2 q}^{(2)}(t) & =K_{2}\left[\left(\int \sin (t)(\cos (t))^{2 q-1} d t\right) \cos (t)-\left(\int(\cos (t))^{2 q} d t\right) \sin (t)\right. \\
& \left.+c_{1} \cos (t)+c_{2} \sin (t)\right]
\end{aligned}
$$

Como, $\forall q \in \mathbb{N}$

$$
\int \sin (t)(\cos (t))^{2 q-1} d t=-\frac{1}{2 q} \cos (t)^{(2 q)}
$$

e

$$
\int(\cos (t))^{2 q} d t=\frac{(2 q-1) ! !}{(2 q) ! !}\left(\sin (t) \sum_{p=0}^{q-1} \frac{(2 p) ! !}{(2 p+1) ! !}(\cos (t))^{2 p+1}+t\right)
$$

então

$$
\begin{aligned}
v_{2 q}^{(2)}(t) & =K_{2}\left(-\frac{1}{2 q}(\cos (t))^{2 q+1}+\frac{1}{2 q} \cos (t)\right) \\
& -K_{2} \frac{(2 q-1) ! !}{(2 q) ! !}\left(\sin (t)^{2} \sum_{p=0}^{q-1} \frac{(2 p) ! !}{(2 p+1) ! !}(\cos (t))^{2 p+1}+t \sin (t)\right)
\end{aligned}
$$

- Resolução do sistema $(S 3)$

$$
\begin{gathered}
\ddot{v}_{2 q}^{(3)}+v_{2 q}^{(3)}=-\frac{1}{3} a_{2 q} \cos (t)^{2 q-1} \cos (2 t) \\
v_{2 q}^{(3)}(0)=0, \quad \dot{v}_{2 q}^{(3)}(0)=0
\end{gathered}
$$


Para todo $q$ natural,

$$
\cos (t)^{2 q-1} \cos (2 t)=2 \cos (t)^{2 q+1)}-\cos (t)^{(2 q-1)} .
$$

Assim, (S3) pode ser reescrito como

$$
\begin{gathered}
\ddot{v}_{2 q}^{(3)}+v_{2 q}^{(3)}=-\frac{1}{3} a_{2 q}\left(2 \cos (t)^{2 q+1}-\cos (t)^{2 q-1}\right) \\
v_{2 q}^{(3)}(0)=0, \quad \dot{v}_{2 q}^{(3)}(0)=0
\end{gathered}
$$

- Resolução do sistema $(S 3)_{1}$

$$
\begin{gathered}
\ddot{v}_{2 q}^{(3)_{1}}+v_{2 q}^{(3)_{1}}=-\frac{2}{3} a_{2 q} \cos (t)^{2 q+1} \\
v_{2 q}^{(3)_{1}}(0)=0, \quad \dot{v}_{2 q}^{(3)_{1}}(0)=0
\end{gathered}
$$

Seja $\left(K_{3}\right)_{1}=\frac{2}{3} a_{2 q}$.

Aplicando o Método da Variação dos Parâmetros a solução do sistema é

$$
\begin{aligned}
v_{2 q}^{(3)_{1}}(t) & =\left(K_{3}\right)_{1}\left[\left(\int \sin (t)(\cos (t))^{2 q+1} d t\right) \cos (t)-\left(\int(\cos (t))^{2 q+2} d t\right) \sin (t)\right. \\
& \left.+c_{1} \cos (t)+c_{2} \sin (t)\right]
\end{aligned}
$$

Como, $\forall q \in \mathbb{N}$

$$
\int \sin (t)(\cos (t))^{2 q+1} d t=-\frac{1}{2 q+2} \cos (t)^{(2 q+2)}
$$

$\mathrm{e}$

$$
\int(\cos (t))^{2 q+2} d t=\frac{(2 q+1) ! !}{(2 q+2) ! !}\left(\sin (t) \sum_{p=0}^{q} \frac{(2 p) ! !}{(2 p+1) ! !}(\cos (t))^{2 p+1}+t\right)
$$

então

$$
\begin{aligned}
v_{2 q}^{(3)_{1}}(t) & =\left(K_{3}\right)_{1}\left(-\frac{1}{2 q+2}(\cos (t))^{2 q+3}+\frac{1}{2 q+2} \cos (t)\right) \\
& -\left(K_{3}\right)_{1} \frac{(2 q+1) ! !}{(2 q+2) ! !}\left(\sin (t)^{2} \sum_{p=0}^{q} \frac{(2 p-1) ! !}{(2 p) ! !}(\cos (t))^{2 p}+t \sin (t)\right)
\end{aligned}
$$

- Resolução do sistema $(S 3)_{2}$

$$
\begin{gathered}
\ddot{v}_{2 q}^{(3)_{2}}+v_{2 q}^{(3)_{2}}=-\left(-\frac{1}{3} a_{2 q}\right) \cos (t)^{2 q-1} \\
v_{2 q}^{(3)_{2}}(0)=0, \quad \dot{v}_{2 q}^{(3)_{2}}(0)=0
\end{gathered}
$$

Seja $\left(K_{3}\right)_{2}=-\frac{1}{3} a_{2 q}$. 
Aplicando o Método da Variação dos Parâmetros a solução do sistema é

$$
\begin{aligned}
v_{2 q}^{(3)_{2}}(t) & =\left(K_{3}\right)_{2}\left[\left(\int \sin (t)(\cos (t))^{2 q-1} d t\right) \cos (t)-\left(\int(\cos (t))^{2 q} d t\right) \sin (t)\right. \\
& \left.+c_{1} \cos (t)+c_{2} \sin (t)\right] .
\end{aligned}
$$

Como, $\forall q \in \mathbb{N}$

$$
\int \sin (t)(\cos (t))^{2 q-1} d t=-\frac{1}{2 q} \cos (t)^{2 q}
$$

$$
\int(\cos (t))^{2 q} d t=\frac{(2 q-1) ! !}{(2 q) ! !}\left(\sin (t) \sum_{p=0}^{q-1} \frac{(2 p) ! !}{(2 p+1) ! !}(\cos (t))^{2 p+1}+t\right)
$$

então

$$
\begin{aligned}
v_{2 q}^{(3)_{2}}(t) & =\left(K_{3}\right)_{2}\left(-\frac{1}{2 q}(\cos (t))^{2 q+1}+\frac{1}{2 q} \cos (t)\right) \\
& -\left(K_{3}\right)_{2} \frac{(2 q-1) ! !}{(2 q) ! !}\left(\sin (t)^{2} \sum_{p=0}^{q-1} \frac{(2 p-1) ! !}{(2 p) ! !}(\cos (t))^{2 p}+t \sin (t)\right)
\end{aligned}
$$

A solução do sistema $(B .2), v_{2 q}(t)$, é a soma das soluções dos sistemas $S 1, S 2,(S 3)_{1}$ e $(S 3)_{2}$.

$$
\begin{aligned}
v_{2 q}(t) & =K_{1}\left(-\frac{1}{2 q+1}(\cos (t))^{2 q+2}+\frac{1}{2 q+1} \cos (t)\right) \\
& -K_{1} \frac{(2 q) ! !}{(2 q+1) ! !}\left((\sin (t))^{2} \sum_{p=0}^{q} \frac{(2 p-1) ! !}{(2 p) ! !}(\cos (t))^{2 p}\right) \\
& +K_{2}\left(-\frac{1}{2 q}(\cos (t))^{2 q+1}+\frac{1}{2 q} \cos (t)\right) \\
& -K_{2} \frac{(2 q-1) ! !}{(2 q) ! !}\left(\sin (t)^{2} \sum_{p=0}^{q-1} \frac{(2 p) ! !}{(2 p+1) ! !}(\cos (t))^{2 p+1}+t \sin (t)\right) \\
& -\left(K_{3}\right)_{1} \frac{(2 q+1) ! !}{(2 q+2) ! !}\left(\sin (t)^{2} \sum_{p=0}^{q} \frac{(2 p-1) ! !}{(2 p) ! !}(\cos (t))^{2 p}+t \sin (t)\right) \\
& +\left(K_{3}\right)_{2}\left(-\frac{1}{2 q}(\cos (t))^{2 q+1}+\frac{1}{2 q} \cos (t)\right) \\
& -\left(K_{3}\right)_{2} \frac{(2 q-1) ! !}{(2 q) ! !}\left(\sin (t)^{2} \sum_{p=0}^{q-1} \frac{(2 p-1) ! !}{(2 p) ! !}(\cos (t))^{2 p}+t \sin (t)\right)
\end{aligned}
$$

Derivando $v_{2 q}(t)$ com respeito a $t$ e calculando em $t=2 \pi$, obtemos

$$
A_{1}^{2 q}=-2 \pi K_{2} \frac{(2 q-1) ! !}{(2 q) ! !}-2 \pi\left(K_{3}\right)_{1} \frac{(2 q+1) ! !}{(2 q+2) ! !}-2 \pi\left(K_{3}\right)_{2} \frac{(2 q-1) ! !}{(2 q) ! !}
$$

Substituindo os valores de $K_{2},\left(K_{3}\right)_{1}$ e $\left(K_{3}\right)_{2}$, temos

$$
A_{1}^{2 q}=-\frac{4 \pi}{3} a_{2 q} \frac{(2 q+1) ! !}{(2 q+2) ! !}+\frac{8 \pi}{3} a_{2 q} \frac{(2 q-1) ! !}{(2 q) ! !}
$$


Simplificando,

$$
A_{1}^{(2 q)}=\frac{4 \pi a_{2 q}}{3}(2 q+3)\left(\frac{(2 q-1) ! !}{(2 q+2) ! !}\right) .
$$

\section{B.2 Demonstração do Lema 6.1.4}

Lema B.2.1. Para todo natural $q$, vale

$$
B_{1}^{2 q}=-\frac{4 \pi a_{2 q}}{3}(2 q+5)\left(\frac{(2 q-3) ! !}{(2 q+2) ! !}\right) .
$$

Demonstração. Devemos encontrar a solução do sistema:

$$
\begin{gathered}
\ddot{u}_{2 q}+u_{2 q}=-a_{2 q} u_{0}(t) \mu_{1}(t)^{2 q-2}(t) \mu_{2}(t) \\
u_{2 q}(0)=0, \quad \dot{u}_{2 q}(0)=0,
\end{gathered}
$$

e calcular em $t=2 \pi$.

Substituindo $u_{0}(t), \mu_{1}(t)$ e $\mu_{2}(t)$, temos

$$
\begin{gathered}
\ddot{u}_{2 q}+u_{2 q}=-a_{2 q} \sin (t) \cos (t)^{(2 q-2)}\left(\frac{2}{3} \cos (t)+\frac{1}{3} \cos (2 t)-1\right) \\
u_{2 q}(0)=0, \quad \dot{u}_{2 q}(0)=0,
\end{gathered}
$$

Reescrevemos o sistema B.4 em três sistemas independentes e depois somamos as soluções de cada um, obtendo $u_{2 q}(t)$.

- Resolução do sistema $(P 1)$

$$
\begin{gathered}
\ddot{u}_{2 q}^{(1)}+u_{2 q}^{(1)}=-\frac{2}{3} a_{2 q} \sin (t) \cos (t)^{(2 q-1)} \\
u_{2 q}^{(1)}(0)=0, \quad \dot{u}_{2 q}^{(1)}(0)=0
\end{gathered}
$$

Aplicando o Método da Variação dos Parâmetros a solução do sistema é

$$
\begin{aligned}
u_{2 q}^{(1)}(t) & =K_{1}\left[\left(\int \sin (t)^{2}(\cos (t))^{2 q-1} d t\right) \cos (t)-\left(\int(\sin (t) \cos (t))^{2 q} d t\right) \sin (t)\right. \\
& \left.+c_{1} \cos (t)+c_{2} \sin (t)\right] \\
& =K_{1}\left[\left(\int \cos (t)^{2 q-1} d t-\int \cos (t)^{2 q+1} d t\right) \cos (t)-\left(\int(\sin (t) \cos (t))^{2 q} d t\right) \sin (t)\right. \\
& \left.+c_{1} \cos (t)+c_{2} \sin (t)\right]
\end{aligned}
$$

Como, $\forall q \in \mathbb{N}$

$$
\int \cos (t)^{2 q-1} d t=\frac{(2 q-2) ! !}{(2 q-1) ! !}\left(\sin (t) \sum_{p=0}^{q-1} \frac{(2 p-1) ! !}{(2 p) ! !}(\cos (t))^{2 p}\right),
$$




$$
\int \cos (t)^{2 q+1} d t=\frac{(2 q) ! !}{(2 q+1) ! !}\left(\sin (t) \sum_{p=0}^{q} \frac{(2 p-1) ! !}{(2 p) ! !}(\cos (t))^{2 p}\right)
$$

e

$$
\int \sin (t) \cos (t)^{2 q} d t=-\frac{1}{2 q+1} \cos (t)^{2 q+1}
$$

temos

$$
\begin{aligned}
u_{2 q}^{(1)}(t) & =K_{1} \frac{(2 q-2) ! !}{(2 q-1) ! !}\left(\sin (t) \sum_{p=0}^{q-1} \frac{(2 p-1) ! !}{(2 p) ! !}(\cos (t))^{2 p}\right) \cos (t) \\
& -K_{1} \frac{(2 q) ! !}{(2 q+1) ! !}\left(\sin (t) \sum_{p=0}^{q} \frac{(2 p-1) ! !}{(2 p) ! !}(\cos (t))^{2 p}\right) \cos (t) \\
& +K_{1}\left(\frac{1}{2 q+1} \cos (t)^{2 q+1} \sin (t)-\frac{1}{2 q+1} \sin (t)\right) \\
& =K_{1}\left[\left(\frac{(2 q-2) ! !}{(2 q-1) ! !}-\frac{(2 q)}{(2 q+1) ! !}\right)\left(\sin (t) \sum_{p=0}^{q-1} \frac{(2 p-1) ! !}{(2 p) ! !}(\cos (t))^{2 p+1}\right)-\frac{1}{2 q+1} \sin (t)\right]
\end{aligned}
$$

- Resolução do sistema (P2)

$$
\begin{gathered}
\ddot{u}_{2 q}^{(2)}+u_{2 q}^{(2)}=-\left(-a_{2 q} \cos (t)^{(2 q-2)}\right) \\
u_{2 q}^{(2)}(0)=0, \quad \dot{u}_{2 q}^{(2)}(0)=0
\end{gathered}
$$

Aplicando o Método da Variação dos Parâmetros a solução do sistema é

$$
\begin{aligned}
u_{2 q}^{(2)}(t) & =K_{2}\left[\left(\int \sin (t)^{2}(\cos (t))^{2 q-2} d t\right) \cos (t)-\left(\int \sin (t)(\cos (t))^{2 q-1} d t\right) \sin (t)\right. \\
& \left.+c_{1} \cos (t)+c_{2} \sin (t)\right],
\end{aligned}
$$

Como, $\forall q \in \mathbb{N}$

$$
\begin{aligned}
& \int \sin (t)(\cos (t))^{2 q-1} d t=-\frac{1}{2 q} \cos (t)^{(2 q)} \\
& \int \sin (t)^{2}(\cos (t))^{2 q-2} d t=\left(\frac{(2 q-3) ! !}{(2 q-2) ! !}-\frac{(2 q-1) ! !}{(2 q) ! !}\right)\left(\sin (t) \sum_{p=0}^{q-1} \frac{(2 p) ! !}{(2 p+1) ! !}(\cos (t))^{2 p+1}+t\right) \\
&-\frac{1}{2 q} \sin (t) \cos (t)^{2 q} .
\end{aligned}
$$

$$
\begin{aligned}
u_{2 q}^{(2)}(t) & =K_{2}\left(\frac{(2 q-3) ! !}{(2 q-2) ! !}-\frac{(2 q-1) ! !}{(2 q) ! !}\right)\left(\cos (t) \sin (t) \sum_{p=0}^{q-1} \frac{(2 p) ! !}{(2 p+1) ! !}(\cos (t))^{2 p+1}+t \cos (t)\right) \\
& -K_{2} \frac{1}{2 q} \sin (t)
\end{aligned}
$$


- Resolução do sistema (P3)

$$
\begin{gathered}
\ddot{u}_{2 q}^{(3)}+u_{2 q}^{(3)}=-\frac{1}{3} a_{2 q} \sin (t) \cos (t)^{2 q-2} \cos (2 t) \\
u_{2 q}^{(3)}(0)=0, \quad \dot{u}_{2 q}^{(3)}(0)=0
\end{gathered}
$$

Para todo $q$ natural,

$$
\cos (t)^{2 q-1} \cos (2 t)=+2 \cos (t)^{2 q)}-\cos (t)^{(2 q-2)},
$$

e $P 3$ pode ser reescrito como

$$
\begin{gathered}
\ddot{u}_{2 q}^{(3)}+u_{2 q}^{(3)}=-\frac{1}{3} a_{2 q} \sin (t)\left(2 \cos (t)^{2 q}-\cos (t)^{2 q-2}\right. \\
u_{2 q}^{(3)}(0)=0, \quad \dot{u}_{2 q}^{(3)}(0)=0
\end{gathered}
$$

- Resolução do sistema $(P 3)_{1}$

$$
\begin{gathered}
\ddot{u}_{2 q}^{(3)_{1}}+u_{2 q}^{(3)_{1}}=-\left(\frac{2}{3} a_{2 q}\right) \sin (t) \cos (t)^{2 q} \\
u_{2 q}^{(3)_{1}}(0)=0, \quad \dot{u}_{2 q}^{(3)_{1}}(0)=0,
\end{gathered}
$$

Aplicando o Método da Variação dos Parâmetros a solução do sistema é

$$
\begin{aligned}
u_{2 q}^{(3)_{1}}(t) & =\left(K_{3}\right)_{1}\left[\left(\int \sin (t)^{2}(\cos (t))^{2 q} d t\right) \cos (t)-\left(\int \sin (t)(\cos (t))^{2 q+1} d t\right) \sin (t)\right. \\
& \left.+c_{1} \cos (t)+c_{2} \sin (t)\right] \\
& =\left(K_{3}\right)_{1}\left[\left(\int(\cos (t))^{2 q} d t-\int(\cos (t))^{2 q+2} d t\right) \cos (t)-\left(\int \sin (t)(\cos (t))^{2 q+1} d t\right) \sin (t)\right. \\
& \left.+c_{1} \cos (t)+c_{2} \sin (t)\right]
\end{aligned}
$$

Como, $\forall q \in \mathbb{N}$

$$
\begin{aligned}
& \int \sin (t)(\cos (t))^{2 q+1} d t=-\frac{1}{2 q+2} \cos (t)^{(2 q+2)} \\
& \int(\cos (t))^{2 q} d t-\int(\cos (t))^{2 q+2} d t=\left(\frac{(2 q-1) ! !}{(2 q) ! !}-\frac{(2 q+1) ! !}{(2 q+2) ! !}\right)\left(\sin (t) \sum_{p=0}^{q-1} \frac{(2 p) ! !}{(2 p+1) ! !}(\cos (t))^{2 p+1}+t\right) \\
&-\frac{1}{2 q+2} \cos (t)^{2 q+1} \sin (t)
\end{aligned}
$$


então

$$
\begin{aligned}
u_{2 q}^{(3)_{1}}(t) & =\left(K_{3}\right)_{1}\left(\frac{(2 q-1) ! !}{(2 q) ! !}-\frac{(2 q+1) ! !}{(2 q+2) ! !}\right)\left(\sin (t) \cos (t) \sum_{p=0}^{q-1} \frac{(2 p) ! !}{(2 p+1) ! !}(\cos (t))^{2 p+1}+t \cos (t)\right) \\
& -\left(K_{3}\right)_{1} \frac{1}{2 q+2} \sin (t)
\end{aligned}
$$

- Resolução do sistema $(P 3)_{2}$

$$
\begin{gathered}
\ddot{u}_{2 q}^{(3)_{2}}+u_{2 q}^{(3)_{2}}=-\left(-\frac{1}{3} a_{2 q}\right) \sin (t) \cos (t)^{2 q-2} \\
u_{2 q}^{(3)_{2}}(0)=0, \quad \dot{u}_{2 q}^{(3)_{2}}(0)=0,
\end{gathered}
$$

Aplicando o Método da Variação dos Parâmetros a solução do sistema é

$$
\begin{aligned}
u_{2 q}^{(3)_{2}}(t) & =\left(K_{3}\right)_{2}\left[\left(\int \sin (t)^{2}(\cos (t))^{2 q-2} d t\right) \cos (t)-\left(\int \sin (t)(\cos (t))^{2 q-1} d t\right) \sin (t)\right. \\
& \left.+c_{1} \cos (t)+c_{2} \sin (t)\right] \\
& +\left(K_{3}\right)_{2}\left[\left(\int(\cos (t))^{2 q-2} d t-\int(\cos (t))^{2 q} d t\right) \cos (t)-\left(\int \sin (t)(\cos (t))^{2 q-1} d t\right) \sin (t)\right. \\
& \left.+c_{1} \cos (t)+c_{2} \sin (t)\right]
\end{aligned}
$$

Como, $\forall q \in \mathbb{N}$

$$
\begin{aligned}
\int \sin (t) & (\cos (t))^{2 q-1} d t=-\frac{1}{2 q} \cos (t)^{(2 q)} \\
\int(\cos (t))^{2 q-2} d t-\int(\cos (t))^{2 q} d t & =\left(\frac{(2 q-3) ! !}{(2 q-2) ! !}-\frac{(2 q-1) ! !}{(2 q) ! !}\right)\left(\sin (t) \sum_{p=0}^{q-2} \frac{(2 p) ! !}{(2 p+1) ! !}(\cos (t))^{2 p+1}+t\right) \\
& -\frac{1}{2 q} \cos (t)^{2 q} \sin (t) .
\end{aligned}
$$

Assim,

$$
\begin{aligned}
u_{2 q}^{(3)_{2}}(t) & =\left(K_{3}\right)_{2}\left(\frac{(2 q-3) ! !}{(2 q-2) ! !}-\frac{(2 q-1) ! !}{(2 q) ! !}\right)\left(\sin (t) \cos (t) \sum_{p=0}^{q-1} \frac{(2 p) ! !}{(2 p+1) ! !}(\cos (t))^{2 p+1}+t \cos (t)\right) \\
& -\left(K_{3}\right)_{2} \frac{1}{2 q} \sin (t)
\end{aligned}
$$


A solução do sistema $(B .4)$ é a soma das soluções dos sistemas $P 1, P 2,(P 3)_{1}$ e $(P 3)_{2}$.

$$
\begin{aligned}
u_{2 q}(t) & =K_{1}\left[\left(\frac{(2 q-2) ! !}{(2 q-1) ! !}-\frac{(2 q)}{(2 q+1) ! !}\right)\left(\sin (t) \sum_{p=0}^{q-1} \frac{(2 p-1) ! !}{(2 p) ! !}(\cos (t))^{2 p+1}\right)-\frac{1}{2 q+1} \sin (t)\right] \\
& +K_{2}\left[\left(\frac{(2 q-3) ! !}{(2 q-2) ! !}-\frac{(2 q-1) ! !}{(2 q) ! !}\right)\left(\cos (t) \sin (t) \sum_{p=0}^{q-1} \frac{(2 p) ! !}{(2 p+1) ! !}(\cos (t))^{2 p+1}+t \cos (t)\right)\right] \\
& -K_{2} \frac{1}{2 q} \sin (t) \\
& +\left(K_{3}\right)_{1}\left(\frac{(2 q-1) ! !}{(2 q) ! !}-\frac{(2 q+1) ! !}{(2 q+2) ! !}\right)\left(\sin (t) \cos (t) \sum_{p=0}^{q-1} \frac{(2 p) ! !}{(2 p+1) ! !}(\cos (t))^{2 p+1}+t \cos (t)\right) \\
& -\left(K_{3}\right)_{1} \frac{1}{2 q+2} \sin (t) \\
& =\left(K_{3}\right)_{2}\left(\frac{(2 q-3) ! !}{(2 q-2) ! !}-\frac{(2 q-1) ! !}{(2 q) ! !}\right)\left(\sin (t) \cos (t) \sum_{p=0}^{q-2} \frac{(2 p) ! !}{(2 p+1) ! !}(\cos (t))^{2 p+1}+t \cos (t)\right) \\
& -\left(K_{3}\right)_{2} \frac{1}{2 q} \sin (t)
\end{aligned}
$$

Calculando $u_{2 q}$ em $t=2 \pi$

$$
\begin{aligned}
B_{1}^{2 q} & =+2 \pi K_{2}\left(\frac{(2 q-3) ! !}{(2 q-2) ! !}-\frac{(2 q-1) ! !}{(2 q) ! !}\right)+2 \pi\left(K_{3}\right)_{1}\left(\frac{(2 q-1) ! !}{(2 q) ! !}-\frac{(2 q+1) ! !}{(2 q+2) ! !}\right) \\
& +2 \pi\left(K_{3}\right)_{2}\left(\frac{(2 q-3) ! !}{(2 q-2) ! !}-\frac{(2 q-1) ! !}{(2 q) ! !}\right)
\end{aligned}
$$

Substituindo os valores de $K_{2},\left(K_{3}\right)_{1}$ e $\left(K_{3}\right)_{2}$, e simplificando, temos

$$
B_{1}^{2 q}=-\frac{8 \pi}{3} a_{2 q} \frac{(2 q-3) ! !}{(2 q-2) ! !}+4 \pi a_{2 q} \frac{(2 q-1) ! !}{(2 q) ! !}-\frac{4 \pi}{3} a_{2 q} \frac{(2 q+1) ! !}{(2 q+2) ! !} .
$$

Simplificando,

$$
B_{1}^{2 q}=-\frac{4 \pi a_{2 q}}{3}(2 q+5)\left(\frac{(2 q-3) ! !}{(2 q+2) ! !}\right)
$$

\section{B.3 Demonstração do Lema 6.1.5}

Lema B.3.1. Para todo q natural, vale

$$
A_{2}^{2 q}=\frac{8 q(2 q+3)}{3}\left(\frac{(2 q-1) ! !}{(2 q+2) ! !}\right)
$$

Demonstração. Para calcular o coeficiente $A_{2}^{2 q}$ devemos resolver o sistema

$$
\begin{aligned}
\ddot{v}_{2 q}+v_{2 q} & =-2 q \mu_{1}(t) v_{1}(t)^{2 q-1} g^{(2 q-1)}(0) \\
& -2 q \mu_{1}(t) \bar{v}_{2 q-1}(t) g^{\prime}(0)
\end{aligned}
$$




$$
v_{(2 q)}(0)=0, \quad \dot{v}_{2 q}(0)=0
$$

em que $\bar{v}_{2 q-1}(t)$ é a solução do sistema

$$
\begin{gathered}
\ddot{\bar{v}}_{2 q-1}+\bar{v}_{2 q-1}=-v_{0}(t) \mu_{1}(t)^{2 q-1}(t) g^{(2 q-1)(0)} \\
\bar{v}_{2 q-1}(0)=0, \quad \dot{\bar{v}}_{2 q-1}(0)=0 .
\end{gathered}
$$

Substituindo os valores de $\mu_{1}(t), v_{1}(t)$ e $\bar{v}_{2 q-1}(t)$ em $B .9$, obtemos

$$
\begin{gathered}
\ddot{\bar{v}}_{2 q-1}+\bar{v}_{2 q-1}=-\cos (t)^{2 q} g^{(2 q-1)}(0) \\
\bar{v}_{2 q-1}(0)=0, \quad \dot{\bar{v}}_{2 q-1}(0)=0,
\end{gathered}
$$

cuja solução é

$$
\begin{aligned}
\bar{v}_{2 q-1}(t) & =g^{(2 q-1)}(0)\left[-\frac{1}{2 q+1} \cos (t)^{2 q+2}+\frac{1}{(2 q+1)} \cos (t)\right. \\
& \left.-\frac{(2 q) ! !}{(2 q+1) ! !}\left(\sin (t)^{2} \sum_{p=0}^{q} \frac{(2 p-1) ! !}{(2 p) ! !}(\cos (t))^{2 p}\right) \cdot\right]
\end{aligned}
$$

Substituindo os valores de $\mu_{1}(t), v_{1}(t)$ e $\bar{v}_{2 q-1}(t)$ em (B.7), temos

$$
\begin{aligned}
\ddot{v}_{2 q}+v_{2 q} & =-2 q \cos (t)^{2 q-1}\left(\frac{1}{3} \cos (t)^{2}-\frac{2}{3}+\frac{1}{3} \cos (t)\right) g^{\prime}(0) g^{(2 q-1)}(0) \\
& -2 q \cos (t)\left(-\frac{1}{2 q+1} \cos (t)^{2 q+2}+\frac{1}{(2 q+1)} \cos (t)\right) g^{\prime}(0) g^{(2 q-1)}(0) \\
& -2 q \cos (t)\left[\frac{(2 q) ! !}{(2 q+1) ! !}\left(\sin (t)^{2} \sum_{p=0}^{q} \frac{(2 p-1) ! !}{(2 p) ! !}(\cos (t))^{2 p}\right)\right] g^{\prime}(0) g^{(2 q-1)}(0) .
\end{aligned}
$$

- Resolução do sistema $S_{i}$

$$
\begin{gathered}
\ddot{v}_{2 q}^{(i)}+v_{2 q}^{(i)}=-\frac{2}{3} q \cos (t)^{2 q+1} \\
v_{(2 q)}^{(i)}(0)=0, \quad \dot{v}_{2 q}^{(i)}(0)=0
\end{gathered}
$$

Seja $L_{1}=\frac{2}{3} q$.

Aplicando o Método da Variação dos Parâmetros a solução do sistema é

$$
\begin{aligned}
v_{2 q}^{(i)}(t) & =L_{1}\left[\left(\int \sin (t)(\cos (t))^{2 q+1} d t\right) \cos (t)-\left(\int(\cos (t))^{2 q+2} d t\right) \sin (t)\right. \\
& \left.+c_{1} \cos (t)+c_{2} \sin (t)\right]
\end{aligned}
$$

Como, $\forall q \in \mathbb{N}$

$$
\int \sin (t)(\cos (t))^{2 q+1} d t=-\frac{1}{2 q+2} \cos (t)^{(2 q+2)}
$$


e

$$
\int(\cos (t))^{2 q+2} d t=\frac{(2 q+1) ! !}{(2 q+2) ! !}\left(\sin (t) \sum_{p=0}^{q} \frac{(2 p) ! !}{(2 p+1) ! !}(\cos (t))^{2 p+1}+t\right)
$$

então,

$$
\begin{aligned}
v_{2 q}^{(i)}(t) & =L_{1}\left(-\frac{1}{2 q+2}(\cos (t))^{2 q+3}+\frac{1}{2 q+2} \cos (t)\right) \\
& -L_{1} \frac{(2 q+1) ! !}{(2 q+2) ! !}\left((\sin (t))^{2} \sum_{p=0}^{q} \frac{(2 p) ! !}{(2 p+1) ! !}(\cos (t))^{2 p+1}+t \sin (t)\right)
\end{aligned}
$$

- Resolução do sistema $\left(S_{i i}\right)$

$$
\begin{gathered}
\ddot{v}_{2 q}^{(i i)}+v_{2 q}^{(i i)}=-\left(-\frac{4}{3} q\right) \cos (t)^{(2 q-1)} \\
v_{2 q}^{(i i)}(0)=0, \quad \dot{v}_{2 q}^{(i i)}(0)=0 .
\end{gathered}
$$

Seja $L_{2}=-\frac{4}{3} q$.

Aplicando o Método da Variação dos Parâmetros a solução do sistema é

$$
\begin{aligned}
v_{2 q}^{(i i)}(t) & =L_{2}\left(-\frac{1}{2 q}(\cos (t))^{2 q+1}+\frac{1}{2 q} \cos (t)\right) \\
& -L_{2} \frac{(2 q-1) ! !}{(2 q) ! !}\left(\sin (t)^{2} \sum_{p=0}^{q-1} \frac{(2 p) ! !}{(2 p+1) ! !}(\cos (t))^{2 p+1}+t \sin (t)\right) .
\end{aligned}
$$

- Resolução do sistema $S_{(i i i)}$

$$
\begin{gathered}
\ddot{v}_{2 q}^{(i i i)}+v_{2 q}^{(i i i)}=-\left(\frac{2}{3} q\right) \cos (t)^{2 q} \\
v_{2 q}^{(i i i)}(0)=0, \quad \dot{v}_{2 q}^{(i i i)}(0)=0
\end{gathered}
$$

Seja $\left(L_{3}\right)=\frac{2}{3} q$

Aplicando o Método da Variação dos Parâmetros a solução do sistema é

$$
\begin{aligned}
v_{2 q}^{(i i i)}(t) & =L_{3}\left[\left(\int \sin (t)(\cos (t))^{2 q} d t\right) \cos (t)-\left(\int(\cos (t))^{2 q+1} d t\right) \sin (t)\right. \\
& \left.+c_{1} \cos (t)+c_{2} \sin (t)\right]
\end{aligned}
$$

Como, $\forall q \in \mathbb{N}$

$$
\int \sin (t)(\cos (t))^{2 q} d t=-\frac{1}{2 q+1} \cos (t)^{(2 q+1)}
$$

e

$$
\int(\cos (t))^{2 q+1} d t=\frac{(2 q) ! !}{(2 q+1) ! !}\left(\sin (t) \sum_{p=0}^{q} \frac{(2 p-1) ! !}{(2 p) ! !}(\cos (t))^{2 p}\right)
$$


então

$$
\begin{aligned}
v_{2 q}^{(i i i)}(t) & =L_{3}\left(-\frac{1}{2 q+1}(\cos (t))^{2 q+2}+\frac{1}{2 q+1} \cos (t)\right) \\
& -L_{3} \frac{(2 q) ! !}{(2 q+1) ! !}\left(\sin (t)^{2} \sum_{p=0}^{q} \frac{(2 p-1) ! !}{(2 p) ! !}(\cos (t))^{2 p}\right)
\end{aligned}
$$

- Resolução do sistema $S_{(i v)}$

$$
\begin{gathered}
\ddot{v}_{2 q}^{(i v)}+v_{2 q}^{(i v)}=-\left(-\frac{2 q}{2 q+1}\right) \cos (t)^{2 q+3} \\
v_{2 q}^{(i v)}(0)=0, \quad \dot{v}_{2 q}^{(i v)}(0)=0
\end{gathered}
$$

Seja $L_{4}=-\frac{2 q}{2 q+1}$.

Aplicando o Método da Variação dos Parâmetros a solução do sistema é

$$
\begin{aligned}
v_{2 q}^{(i v)}(t) & =L_{4}\left[\left(\int \sin (t)(\cos (t))^{2 q+3} d t\right) \cos (t)-\left(\int(\cos (t))^{2 q+4} d t\right) \sin (t)\right. \\
& \left.+c_{1} \cos (t)+c_{2} \sin (t)\right]
\end{aligned}
$$

Como, $\forall q \in \mathbb{N}$

$$
\int \sin (t)(\cos (t))^{2 q+3} d t=-\frac{1}{2 q+4} \cos (t)^{2 q+4}
$$

$$
\int(\cos (t))^{2 q+4} d t=\frac{(2 q+3) ! !}{(2 q+4) ! !}\left(\sin (t) \sum_{p=0}^{q+1} \frac{(2 p) ! !}{(2 p+1) ! !}(\cos (t))^{2 p+1}+t\right)
$$

então

$$
\begin{aligned}
v_{2 q}^{(i v)}(t) & =L_{4}\left(-\frac{1}{2 q+4}(\cos (t))^{2 q+5}+\frac{1}{2 q+4} \cos (t)\right) \\
& -L_{4} \frac{(2 q+3) ! !}{(2 q+4) ! !}\left(\sin (t)^{2} \sum_{p=0}^{q-1} \frac{(2 p-1) ! !}{(2 p) ! !}(\cos (t))^{2 p}+t \sin (t)\right) .
\end{aligned}
$$

- Resolução do sistema $S_{(v)}$

$$
\begin{gathered}
\ddot{v}_{2 q}^{(v)}+v_{2 q}^{(v)}=-\left(\frac{2 q}{2 q+1}\right) \cos (t)^{2} \\
v_{2 q}^{(v)}(0)=0, \quad \dot{v}_{2 q}^{(v)}(0)=0 .
\end{gathered}
$$

Seja $L_{5}=\frac{2 q}{2 q+1}$.

Aplicando o Método da Variação dos Parâmetros a solução do sistema é 


$$
\begin{aligned}
v_{2 q}^{(v)}(t) & =L_{5}\left(-\frac{1}{3}(\cos (t))^{4}+\frac{1}{3} \cos (t)\right) \\
& -L_{5} \frac{(6) ! !}{(5) ! !}\left(\sin (t)^{2} \sum_{p=0}^{1} \frac{(2 p-1) ! !}{(2 p) ! !}(\cos (t))^{2 p}\right) .
\end{aligned}
$$

- Resolução do sistema $S_{(v i)}$

$$
\begin{gathered}
\ddot{v}_{2 q}^{(v i)}+v_{2 q}^{(v i)}=-\left(-2 q \frac{(2 q) ! !}{(2 q+1) ! !}\right) \cos (t)\left(\sin (t)^{2} \sum_{p=0}^{q} \frac{(2 p-1) ! !}{(2 p) ! !}(\cos (t))^{2 p}\right) \\
=-\left(-2 q \frac{(2 q) ! !}{(2 q+1) ! !}\right)\left(\left(\cos (t)-\cos (t)^{3}\right) \sum_{p=0}^{q} \frac{(2 p-1) ! !}{(2 p) ! !}(\cos (t))^{2 p}\right) \\
v_{2 q}^{(v i)}(0)=0, \quad \dot{v}_{2 q}^{(v i)}(0)=0 .
\end{gathered}
$$

Seja $L_{6}=-2 q \frac{(2 q) ! !}{(2 q+1) ! !}$

- Resolução de $S_{(v i)_{1}}$

$$
\begin{gathered}
\ddot{v}_{2 q}^{(v i)_{1}}+v_{2 q}^{(v i)_{1}}=-L_{6} \cos (t)\left(\sum_{p=0}^{q} \frac{(2 p-1) ! !}{(2 p) ! !}(\cos (t))^{2 p}\right) \\
v_{2 q}^{(v i)_{1}}(0)=0, \quad \dot{v}_{2 q}^{(v i)_{1}}(0)=0 .
\end{gathered}
$$

Aplicando o Método da Variação dos Parâmetros a solução do sistema é

$$
\begin{aligned}
v_{2 q}^{(v i)_{1}}(t) & =L_{6}\left[\int \sin (t) \cos (t)\left(\sum_{p=0}^{q} \frac{(2 p-1) ! !}{(2 p) ! !}(\cos (t))^{2 p}\right) d t\right] \cos (t) \\
& -L_{6}\left[\int \cos (t)^{2}\left(\sum_{p=0}^{q} \frac{(2 p-1) ! !}{(2 p) ! !}(\cos (t))^{2 p}\right) d t\right] \sin (t) \\
& +c_{1} \cos (t)+c_{2} \sin (t) .
\end{aligned}
$$

$$
\begin{aligned}
v_{2 q}^{(v i)_{1}}(t) & =L_{6}\left(\sum_{p=0}^{q} \frac{(2 p-1) ! !}{(2 p) ! !} \int \sin (t)(\cos (t))^{2 p+1} d t\right) \cos (t) \\
& -L_{6}\left(\sum_{p=0}^{q} \frac{(2 p-1) ! !}{(2 p) ! !} \int(\cos (t))^{2 p+2} d t\right) \sin (t) \\
& +c_{1} \cos (t)+c_{2} \sin (t)
\end{aligned}
$$


Como, $\forall q \in \mathbb{N}$

$$
\int \sin (t)(\cos (t))^{2 p+1} d t=-\frac{1}{2 p+2} \cos (t)^{2 p+2}
$$

$$
\int(\cos (t))^{2 p+2} d t=\frac{(2 p+1) ! !}{(2 p+2) ! !}\left(\sin (t) \sum_{l=0}^{p} \frac{(2 l) ! !}{(2 l+1) ! !}(\cos (t))^{2 l+1}+t\right) .
$$

Assim,

$$
\begin{aligned}
v_{2 q}^{(v i)_{1}}(t) & =L_{6}\left[-\sum_{p=0}^{q} \frac{(2 p-1) ! !}{(2 p) ! !} \frac{1}{2 p+2} \cos (t)^{2 p+3}+\sum_{p=0}^{q} \frac{(2 p-1) ! !}{(2 p) ! !} \frac{1}{2 p+2} \cos (t)\right] \\
& -L_{6}\left[\sum_{p=0}^{q} \frac{(2 p-1) ! !}{(2 p) ! !} \frac{(2 p+1) ! !}{(2 p+2) ! !}\left(\sin (t)^{2} \sum_{l=0}^{p} \frac{(2 l) ! !}{(2 l+1) ! !}(\cos (t))^{2 l+1}+t \sin (t)\right)\right]
\end{aligned}
$$

- Resolução de $S_{(v i)_{2}}$

$$
\begin{gathered}
\ddot{v}_{2 q}^{(v i)_{2}}+v_{2 q}^{(v i)_{2}}=-L_{6}\left(-\cos (t)^{3}\right)\left(\sum_{p=0}^{q} \frac{(2 p-1) ! !}{(2 p) ! !}(\cos (t))^{2 p}\right) \\
v_{2 q}^{(v i)_{2}}(0)=0, \quad \dot{v}_{2 q}^{(v i)_{2}}(0)=0 .
\end{gathered}
$$

Aplicando o Método da Variação dos Parâmetros a solução do sistema é

$$
\begin{aligned}
v_{2 q}^{(v i)_{2}}(t) & =-L_{6}\left[\int \sin (t) \cos (t)^{3}\left(\sum_{p=0}^{q} \frac{(2 p-1) ! !}{(2 p) ! !}(\cos (t))^{2 p}\right) d t\right] \cos (t) \\
& +L_{6}\left[\int \cos (t)^{4}\left(\sum_{p=0}^{q} \frac{(2 p-1) ! !}{(2 p) ! !}(\cos (t))^{2 p}\right) d t\right] \sin (t) \\
& +c_{1} \cos (t)+c_{2} \sin (t) .
\end{aligned}
$$

$$
\begin{aligned}
v_{2 q}^{(v i)_{2}}(t) & =-L_{6}\left(\sum_{p=0}^{q} \frac{(2 p-1) ! !}{(2 p) ! !} \int \sin (t)(\cos (t))^{2 p+3} d t\right) \cos (t) \\
& +L_{6}\left(\sum_{p=0}^{q} \frac{(2 p-1) ! !}{(2 p) ! !} \int(\cos (t))^{2 p+4} d t\right) \sin (t) \\
& +c_{1} \cos (t)+c_{2} \sin (t) .
\end{aligned}
$$

Como, $\forall q \in \mathbb{N}$

$$
\int \sin (t)(\cos (t))^{2 p+3} d t=-\frac{1}{2 p+4} \cos (t)^{2 p+4}
$$


e

$$
\int(\cos (t))^{2 p+4} d t=\frac{(2 p+3) ! !}{(2 p+4) ! !}\left(\sin (t) \sum_{l=0}^{p+1} \frac{(2 l) ! !}{(2 l+1) ! !}(\cos (t))^{2 l+1}+t\right)
$$

então

$$
\begin{aligned}
v_{2 q}^{(v i)}(t) & =L_{6}\left[\sum_{p=0}^{q} \frac{(2 p-1) ! !}{(2 p) ! !} \frac{1}{2 p+4} \cos (t)^{2 p+5}-\sum_{p=0}^{q} \frac{(2 p-1) ! !}{(2 p) ! !} \frac{1}{2 p+4} \cos (t)\right] \\
& +L_{6}\left[\sum_{p=0}^{q} \frac{(2 p-1) ! !}{(2 p) ! !} \frac{(2 p+3) ! !}{(2 p+4) ! !}\left(\sin (t)^{2} \sum_{l=0}^{p+1} \frac{(2 l) ! !}{(2 l+1) ! !}(\cos (t))^{2 l+1}+t \sin (t)\right)\right]
\end{aligned}
$$


A solução do sistema (B.7) é a soma das soluções dos sistemas $S_{i}, S_{i i}, S_{i i i}, S_{i v}, S_{v}, S_{(v i)_{1}}$ e $S_{(v i)_{2}}$.

$$
\begin{aligned}
& v_{2 q}(t)=L_{1}\left(-\frac{1}{2 q+2}(\cos (t))^{2 q+3}+\frac{1}{2 q+2} \cos (t)\right) \\
& \text { - } L_{1} \frac{(2 q+1) ! !}{(2 q+2) ! !}\left((\sin (t))^{2} \sum_{p=0}^{q} \frac{(2 p) ! !}{(2 p+1) ! !}(\cos (t))^{2 p+1}+t \sin (t)\right) \\
& +L_{2}\left(-\frac{1}{2 q}(\cos (t))^{2 q+1}+\frac{1}{2 q} \cos (t)\right) \\
& -\quad L_{2} \frac{(2 q-1) ! !}{(2 q) ! !}\left(\sin (t)^{2} \sum_{p=0}^{q-1} \frac{(2 p) ! !}{(2 p+1) ! !}(\cos (t))^{2 p+1}+t \sin (t)\right) \\
& +L_{3}\left(-\frac{1}{2 q+1}(\cos (t))^{2 q+2}+\frac{1}{2 q+1} \cos (t)\right) \\
& \text { - } L_{3} \frac{(2 q) ! !}{(2 q+1) ! !}\left(\sin (t)^{2} \sum_{p=0}^{q} \frac{(2 p-1) ! !}{(2 p) ! !}(\cos (t))^{2 p}\right) \\
& +L_{4}\left(-\frac{1}{2 q+4}(\cos (t))^{2 q+5}+\frac{1}{2 q+4} \cos (t)\right) \\
& \text { - } L_{4} \frac{(2 q+3) ! !}{(2 q+4) ! !}\left(\sin (t)^{2} \sum_{p=0}^{q-1} \frac{(2 p-1) ! !}{(2 p) ! !}(\cos (t))^{2 p}+t \sin (t)\right) \\
& +\quad L_{5}\left(-\frac{1}{3}(\cos (t))^{4}+\frac{1}{3} \cos (t)\right) \\
& \text { - } L_{5} \frac{(6) ! !}{(5) ! !}\left(\sin (t)^{2} \sum_{p=0}^{1} \frac{(2 p-1) ! !}{(2 p) ! !}(\cos (t))^{2 p}\right) \\
& +\quad L_{6}\left[-\sum_{p=0}^{q} \frac{(2 p-1) ! !}{(2 p) ! !} \frac{1}{2 p+2} \cos (t)^{2 p+3}+\sum_{p=0}^{q} \frac{(2 p-1) ! !}{(2 p) ! !} \frac{1}{2 p+2} \cos (t)\right] \\
& \text { - } L_{6}\left[\sum_{p=0}^{q} \frac{(2 p-1) ! !}{(2 p) ! !} \frac{(2 p+1) ! !}{(2 p+2) ! !}\left(\sin (t)^{2} \sum_{l=0}^{p} \frac{(2 l) ! !}{(2 l+1) ! !}(\cos (t))^{2 l+1}+t \sin (t)\right)\right] \\
& +\quad L_{6}\left[\sum_{p=0}^{q} \frac{(2 p-1) ! !}{(2 p) ! !} \frac{1}{2 p+4} \cos (t)^{2 p+5}-\sum_{p=0}^{q} \frac{(2 p-1) ! !}{(2 p) ! !} \frac{1}{2 p+4} \cos (t)\right] \\
& +\quad L_{6}\left[\sum_{p=0}^{q} \frac{(2 p-1) ! !}{(2 p) ! !} \frac{(2 p+3) ! !}{(2 p+4) ! !}\left(\sin (t)^{2} \sum_{l=0}^{p+1} \frac{(2 l) ! !}{(2 l+1) ! !}(\cos (t))^{2 l+1}+t \sin (t)\right)\right] .
\end{aligned}
$$

Derivando $v_{2 q}(t)$ com respeito a $t$, calculando em $t=2 \pi$ e substituindo os valores $L_{i}, 1 \leq i \leq 6$, segue que

$$
\begin{aligned}
A_{2}^{2 q} & =-\frac{4 q \pi}{3}\left(\frac{(2 q+1) ! !}{(2 q+2) ! !}\right)+\frac{8 q \pi}{3}\left(\frac{(2 q-1) ! !}{(2 q) ! !}\right)+\frac{4 q \pi}{2 q+1}\left(\frac{(2 q+3) ! !}{(2 q+4) ! !}\right) \\
& -4 q \pi\left(\frac{(2 q) ! !}{(2 q+1) ! !}\right) \sum_{p=0}^{q} \frac{(2 p-1) ! !}{(2 p) ! !}\left(\frac{(2 q+3) ! !}{(2 q+4) ! !}-\frac{(2 q+1) ! !}{(2 q+2) ! !}\right)
\end{aligned}
$$


Vamos simplificar essa expressão

$$
\begin{aligned}
A_{2}^{2 q}= & \frac{4 q \pi}{3}\left(-\frac{(2 q+1) ! !}{(2 q+2) ! !}+2 \frac{(2 q-1) ! !}{(2 q) ! !}\right)+\frac{4 q \pi}{(2 q+1)} \frac{(2 q+3) ! !}{(2 q+4) ! !} \\
- & 4 q \pi \frac{(2 q) ! !}{(2 q+1) ! !} \sum_{p=0}^{q-1} \frac{(2 p-1) ! !}{(2 p) ! !}\left(\frac{(2 p+3) ! !}{(2 p+4) ! !}-\frac{(2 p+1) ! !}{(2 p+2) ! !}\right) \\
- & 4 q \pi \frac{(2 q) ! !}{(2 q+1) ! !} \frac{(2 q-1) ! !}{(2 q) ! !} \frac{(2 q+3) ! !}{(2 q+4) ! !}+4 q \pi \frac{(2 q) ! !}{(2 q+1) ! !} \frac{(2 q-1) ! !}{(2 q) ! !} \frac{(2 q+1) ! !}{(2 q+2) ! !} \\
= & \frac{4 q \pi}{3}\left(-\frac{(2 q+1) ! !+2(2 q-1) ! !}{(2 q+2) ! !}\right)+4 q \pi \frac{(2 q) ! !}{(2 q+1) ! !} \frac{(2 q-1) ! !}{(2 q) ! !} \frac{(2 q+1) ! !}{(2 q+2) ! !} \\
- & 4 q \pi \sum_{p=0}^{q-1} \frac{(2 p-1) ! !}{(2 p) ! !}\left(\frac{(2 p+3) ! !}{(2 p+4) ! !}-\frac{(2 p+1) ! !}{(2 p+2) ! !}\right) \\
= & \frac{4 q \pi}{3}(2 q+3)\left(\frac{(2 q-1) ! !}{(2 q+2) ! !}\right)+4 q \pi\left(\frac{(2 q-1) ! !}{(2 q+2) ! !}\right)+4 q \pi \sum_{p=0}^{q-1} \frac{(2 p-1) ! !}{(2 p) ! !}\left(\frac{(2 p+1) ! !}{(2 p+4) ! !}\right) \\
& A_{2}^{(2 q)}=\frac{4 q \pi}{3}(2 q+6)\left(\frac{(2 q-1) ! !}{(2 q+2) ! !}\right)+4 q \pi \sum_{p=0}^{q-1} \frac{(2 p-1) ! !}{(2 p) ! !}\left(\frac{(2 p+1) ! !}{(2 p+4) ! !}\right) .
\end{aligned}
$$

Para completar a demonstração, utilizamos:

Afirmação B.3.1. Para todo $q \geq 2 \in \mathbb{N}$,

$$
\frac{2 q}{3}\left(\frac{(2 q-1) ! !}{(2 q+2) ! !}\right)=\frac{(2 q) ! !}{(2 q+1) ! !} \sum_{p=0}^{q-1} \frac{(2 p-1) ! !}{(2 p) ! !}\left(\frac{(2 p+1) ! !}{(2 p+4) ! !}\right)
$$

De (B.10) e da Afirmação (B.3.1),

$$
A_{2}^{2 q}=\frac{8 q(2 q+3)}{3}\left(\frac{(2 q-1) ! !}{(2 q+2) ! !}\right) .
$$

\section{B.4 Demonstração do Lema 6.1.6}

Lema B.4.1. Para todo q natural, vale

$$
B_{2}^{2 q}=+\frac{8 q \pi}{3}\left(\frac{(2 q-1) ! !}{(2 q+2) ! !}\right) .
$$

Demonstração. Vamos calcular o valor de $B_{2}^{2 q}$, para isso devemos encontrar a solução do sistema:

$$
\begin{gathered}
\ddot{u}_{2 q}(t)+u_{2 q}(t)= \\
-2 q \mu_{1}(t)^{(2 q-1)} u_{1}(t) g^{(2 q-1)}(0) \\
-2 q \mu_{1}(t) \bar{u}_{2 q-1}(t) g^{\prime}(0) . \\
u_{2 q}(0)=0, \quad \dot{u}_{2 q}(0)=0 .
\end{gathered}
$$


em que $\bar{u}_{2 q-1}(t)$ é a solução do sistema

$$
\begin{gathered}
\ddot{\bar{u}}_{(2 q-1)}(t)+\bar{u}_{(2 q-1)}(t)=u_{0}(t) \mu_{1}(t)^{(2 q-1)} g^{(2 q-1)}(0) \\
\bar{u}_{2 q-1}(0)=0, \quad \dot{\bar{u}}_{2 q-1}(0)=0 .
\end{gathered}
$$

Substituindo os valores de $u_{0}(t)$ e $\mu_{1}(t)$ em (B.14), obtemos

$$
\begin{gathered}
\ddot{\bar{u}}_{2 q-1}+u_{2 q-1}=-\sin (t) \cos (t)^{2 q-1}(t) g^{(2 q-1)(0)} \\
\bar{u}_{2 q-1}(0)=0, \quad \dot{\bar{u}}_{2 q-1}(0)=0,
\end{gathered}
$$

cuja solução é

$$
\bar{u}_{2 q-1}=g^{(2 q-1)}(0)\left[\left(\frac{(2 q-2) ! !}{(2 q-1) ! !}-\frac{(2 q) ! !}{(2 q+1) ! !}\right) \sin (t) \sum_{p=0}^{q-1} \frac{(2 p-1) ! !}{(2 p) ! !} \cos (t)^{2 p+1}-\frac{1}{q+1} \sin (t)\right] .
$$

Substituindo os valores de $u_{1}(t), \mu_{1}(t)$ e $\bar{u}_{2 q-1}$ em $(B .13)$

$$
\begin{aligned}
\ddot{u}_{2 q}+u_{2 q} & =-2 q \cos (t)^{(2 q-1)}\left(\frac{1}{3} \cos (t) \sin (t)-\frac{1}{3} \sin (t)\right) g^{\prime}(0) g^{(2 q-1)}(0) \\
& -2 q \cos (t)\left[\left(\frac{(2 q-2) ! !}{(2 q-1) ! !}-\frac{(2 q) ! !}{(2 q+1) ! !}\right) \sin (t) \sum_{p=0}^{q-1} \frac{(2 p-1) ! !}{(2 p) ! !} \cos (t)^{2 k+1}-\frac{1}{q+1} \sin (t)\right] \\
& \times g^{\prime}(0) g^{(2 q-1)}(0)
\end{aligned}
$$

- Resolução do sistema $P_{(i)}$

$$
\begin{gathered}
\ddot{u}_{2 q}^{(i)}+u_{2 q}^{(i)}=-\frac{2 q}{3} \sin (t) \cos (t)^{(2 q)} \\
u_{2 q}^{(2)}(0)=0, \quad \dot{u}_{2 q}^{(2)}(0)=0,
\end{gathered}
$$

Seja $M_{1}=\frac{2 q}{3}$.

Aplicando o Método da Variação dos Parâmetros a solução do sistema é

$$
\begin{aligned}
u_{2 q}^{(i)}(t) & =M_{1}\left[\left(\int \sin (t)^{2}(\cos (t))^{2 q} d t\right) \cos (t)-\left(\int \sin (t)(\cos (t))^{2 q+1} d t\right) \sin (t)\right. \\
& \left.+c_{1} \cos (t)+c_{2} \sin (t)\right],
\end{aligned}
$$

Como, $\forall q \in \mathbb{N}$

$$
\int \sin (t)(\cos (t))^{2 q+1} d t=-\frac{1}{2 q+2} \cos (t)^{(2 q+2)}
$$

e

$$
\begin{aligned}
\int \sin (t)^{2}(\cos (t))^{2 q} d t & =\left(\frac{(2 q-1) ! !}{(2 q) ! !}-\frac{(2 q+1) ! !}{(2 q+2) ! !}\right)\left(\sin (t) \sum_{p=0}^{q-1} \frac{(2 p) ! !}{(2 p+1) ! !}(\cos (t))^{2 p+1}+t\right) \\
& -\frac{1}{2 q+2} \sin (t) \cos (t)^{2 q+1}
\end{aligned}
$$


Assim,

$$
\begin{aligned}
u_{2 q}^{(i)}(t) & =M_{1}\left[\left(\frac{(2 q-1) ! !}{(2 q) ! !}-\frac{(2 q+1) ! !}{(2 q+2) ! !}\right)\left(\sin (t) \sum_{p=0}^{q-1} \frac{(2 p) ! !}{(2 p+1) ! !}(\cos (t))^{2 p+1}+t\right)\right] \cos (t) \\
& =-M_{1}\left[\frac{1}{2 q+2} \sin (t) \cos (t)^{2 q+2}+\frac{1}{2 q+2} \sin (t) \cos (t)^{2 q+2}-\frac{1}{2 q+2} \sin (t)\right]
\end{aligned}
$$

- Resolução do sistema $P_{(i i)}$

$$
\begin{gathered}
\ddot{u}_{2 q}^{(i i)}+u_{2 q}^{(i i)}=-\left(-\frac{2 q}{3} \sin (t) \cos (t)^{(2 q-1)}\right) \\
u_{2 q}^{(2)}(0)=0, \quad \dot{u}_{2 q}^{(2)}(0)=0,
\end{gathered}
$$

Aplicando o Método da Variação dos Parâmetros a solução do sistema é

$$
\begin{aligned}
u_{2 q}^{(2)}(t) & =-M_{1}\left[\left(\int \sin (t)^{2}(\cos (t))^{2 q-1} d t\right) \cos (t)-\left(\int \sin (t)(\cos (t))^{2 q} d t\right) \sin (t)\right. \\
& \left.+c_{1} \cos (t)+c_{2} \sin (t)\right]
\end{aligned}
$$

Como, $\forall q \in \mathbb{N}$

$$
\int \sin (t)(\cos (t))^{2 q} d t=-\frac{1}{2 q+1} \cos (t)^{(2 q+1)}
$$

e

$$
\begin{aligned}
\int \sin (t)^{2}(\cos (t))^{2 q-1} d t & =\left(\frac{(2 q-2) ! !}{(2 q-1) ! !}-\frac{(2 q) ! !}{(2 q+1) ! !}\right)\left(\sin (t) \sum_{p=0}^{q-1} \frac{(2 p-1) ! !}{(2 p) ! !}(\cos (t))^{2 p}\right) \\
& -\frac{1}{2 q+2} \sin (t) \cos (t)^{2 q+1}
\end{aligned}
$$

Assim,

$$
\begin{aligned}
u_{2 q}^{(i i)}(t) & =-M_{1}\left[\left(\frac{(2 q-2) ! !}{(2 q-1) ! !}-\frac{(2 q) ! !}{(2 q+1) ! !}\right)\left(\sin (t) \sum_{p=0}^{q-1} \frac{(2 p-1) ! !}{(2 p) ! !}(\cos (t))^{2 p}\right)\right] \cos (t) \\
& =-M_{1}\left(-\frac{1}{2 q+1} \sin (t) \cos (t)^{2 q+1}+\frac{1}{2 q+1} \sin (t) \cos (t)^{2 q+1}-\frac{1}{2 q+1} \sin (t)\right)
\end{aligned}
$$

- Resolução do sistema $P_{(i i i)}$

$$
\begin{gathered}
\ddot{u}_{2 q}^{(i i i)}+u_{2 q}^{(i i i)}=-2 q \cos (t)\left(\frac{(2 q-2) ! !}{(2 q-1) ! !}-\frac{(2 q) ! !}{(2 q+1) ! !}\right) \sin (t) \sum_{p=0}^{q-1} \frac{(2 p-1) ! !}{(2 p) ! !} \cos (t)^{2 p+1} \\
u_{2 q}^{(2)}(0)=0, \quad \dot{u}_{2 q}^{(2)}(0)=0 .
\end{gathered}
$$

Seja $M_{3}=2 q\left(\frac{(2 q-2) ! !}{(2 q-1) ! !}-\frac{(2 q) ! !}{(2 q+1) ! !}\right)$. 
Aplicando o Método da Variação dos Parâmetros a solução do sistema é

$$
\begin{aligned}
u_{2 q}^{(i i i)}(t) & =M_{3}\left[\left(\int \sin (t)^{2} \sum_{p=0}^{q-1} \frac{(2 p-1) ! !}{(2 p) ! !} \cos (t)^{2 p+2} d t\right) \cos (t)\right. \\
& -\left(\int \sin (t) \sum_{p=0}^{q-1} \frac{(2 p-1) ! !}{(2 p) ! !} \cos (t)^{2 p+3} d t\right) \sin (t) \\
& \left.+c_{1} \cos (t)+c_{2} \sin (t)\right],
\end{aligned}
$$

que pode ser reescrito como:

$$
\begin{aligned}
u_{2 q}^{(i i i)}(t) & =M_{3}\left[\left(\sum_{p=0}^{q-1} \frac{(2 p-1) ! !}{(2 p) ! !} \int \sin (t)^{2} \cos (t)^{2 p+2} d t\right) \cos (t)\right. \\
& -\left(\sum_{p=0}^{q-1} \frac{(2 p-1) ! !}{(2 p) ! !} \int \sin (t) \cos (t)^{2 p+3} d t\right) \sin (t) \\
& \left.+c_{1} \cos (t)+c_{2} \sin (t)\right]
\end{aligned}
$$

Como, $\forall q \in \mathbb{N}$

$$
\int \sin (t)(\cos (t))^{2 p+3} d t=-\frac{1}{2 p+4} \cos (t)^{(2 p+3)}
$$

e

$$
\begin{aligned}
\int \sin (t)^{2}(\cos (t))^{2 p+2} d t & =\left(\frac{(2 p+1) ! !}{(2 p+2) ! !}-\frac{(2 p+3) ! !}{(2 p+4) ! !}\right)\left(\sin (t) \sum_{l=0}^{p} \frac{(2 l) ! !}{(2 l+1) ! !}(\cos (t))^{2 l+1}+t\right) \\
& +\frac{1}{2 p+4} \sin (t) \cos (t)^{2 p+3}
\end{aligned}
$$

então

$$
\begin{aligned}
u_{2 q}^{(i i i)}(t) & =M_{3}\left[\sum_{p=0}^{q-1} \frac{(2 p-1) ! !}{(2 p) ! !}\left(\frac{(2 p+1) ! !}{(2 p+2) ! !}-\frac{(2 p+3) ! !}{(2 p+4) ! !}\right) \sin (t) \sum_{l=0}^{p} \frac{(2 l) ! !}{(2 l+1) ! !}(\cos (t))^{2 l+1}+t\right] \cos (t) \\
& +M_{3}\left(\sum_{p=0}^{q-1} \frac{(2 p-1) ! !}{(2 p) ! !} \frac{1}{2 p+4} \sin (t) \cos (t)^{2 p+4}-\sum_{p=0}^{q-1} \frac{(2 p-1) ! !}{(2 p) ! !} \frac{1}{2 p+4} \sin (t) \cos (t)^{2 p+4}\right) \\
& +\sum_{p=0}^{q-1} \frac{(2 p-1) ! !}{(2 p) ! !} \frac{1}{2 p+4} \sin (t)
\end{aligned}
$$

A solução do sistema $B .13$ é a soma das soluções dos sistemas $P_{(i)}, P_{(i i)}$ e $P_{(i i i)}$ ou seja 


$$
\begin{aligned}
u_{2 q}(t) & =M_{1}\left[\left(\frac{(2 q-1) ! !}{(2 q) ! !}-\frac{(2 q+1) ! !}{(2 q+2) ! !}\right)\left(\sin (t) \sum_{p=0}^{q-1} \frac{(2 p) ! !}{(2 p+1) ! !}(\cos (t))^{2 p+1}+t\right)\right] \cos (t) \\
& -M_{1}\left[\frac{1}{2 q+2} \sin (t) \cos (t)^{2 q+2}+\frac{1}{2 q+2} \sin (t) \cos (t)^{2 q+2}-\frac{1}{2 q+2} \sin (t)\right] \\
& -M_{1}\left[\left(\frac{(2 q-2) ! !}{(2 q-1) ! !}-\frac{(2 q) ! !}{(2 q+1) ! !}\right)\left(\sin (t) \sum_{p=0}^{q-1} \frac{(2 p-1) ! !}{(2 p) ! !}(\cos (t))^{2 p}\right)\right] \cos (t) \\
& -M_{1}\left(-\frac{1}{2 q+1} \sin (t) \cos (t)^{2 q+1}+\frac{1}{2 q+1} \sin (t) \cos (t)^{2 q+1}-\frac{1}{2 q+1} \sin (t)\right) \\
& +M_{3}\left[\sum_{p=0}^{q-1} \frac{(2 p-1) ! !}{(2 p) ! !}\left(\frac{(2 p+1) ! !}{(2 p+2) ! !}-\frac{(2 p+3) ! !}{(2 p+4) ! !}\right) \sin (t) \sum_{l=0}^{p} \frac{(2 l) ! !}{(2 l+1) ! !}(\cos (t))^{2 l+1}+t\right] \cos (t) \\
& +M_{3}\left(\sum_{p=0}^{q-1} \frac{(2 p-1) ! !}{(2 p) ! !} \frac{1}{2 p+4} \sin (t) \cos (t)^{2 p+4}-\sum_{p=0}^{q-1} \frac{(2 p-1) ! !}{(2 p) ! !} \frac{1}{2 p+4} \sin (t) \cos (t)^{2 p+4}\right) \\
& +\sum_{p=0}^{q-1} \frac{(2 p-1) ! !}{(2 p) ! !} \frac{1}{2 p+4} \sin (t)
\end{aligned}
$$

Calculando em $t=2 \pi$ e substituindo os valores de $M_{1}$ e $M_{3}$, temos

$$
\begin{aligned}
B_{2}^{2 q} & =4 q \pi\left(\frac{(2 q-2) ! !}{(2 q-1) ! !}-\frac{(2 q) ! !}{(2 q+1) ! !}\right) \sum_{p=0}^{q-1} \frac{(2 p-1) ! !}{(2 p) ! !}\left(\frac{(2 p+1) ! !}{(2 p+2) ! !}-\frac{(2 p+3) ! !}{(2 p+4) ! !}\right) \\
& +\frac{4 q \pi}{3}\left(\frac{(2 q-1) ! !}{(2 q) ! !}-\frac{(2 q+1) ! !}{(2 q+2) ! !}\right) \\
& =4 q \pi\left(\frac{(2 q-2) ! !(2 q-1) ! !-(2 q) ! !}{(2 q+1) ! !}\right) \sum_{p=0}^{q-1} \frac{(2 p-1) ! !}{(2 p) ! !} \frac{(2 p+1) ! !}{(2 p+4) ! !} \\
& +\frac{4 q \pi}{3}\left(\frac{(2 q+2) ! !(2 q-1) ! !-(2 q+1) ! !}{(2 q+2) ! !}\right) \\
& B_{2}^{2 q}=4 q \pi\left(\frac{(2 q-2) ! !}{(2 q+1) ! !}\right) \sum_{p=0}^{q-1} \frac{(2 p-1) ! !}{(2 p) ! !} \frac{(2 p+1) ! !}{(2 p+4) ! !}+\frac{4 q \pi}{3}\left(\frac{(2 q-1) ! !}{(2 q+2)}\right) .
\end{aligned}
$$

Afirmação B.4.1. Para todo $q \geq 2 \in \mathbb{N}$,

$$
\frac{1}{3}\left(\frac{(2 q-1) ! !}{(2 q+2) ! !}\right)=\frac{(2 q-2) ! !}{(2 q+1) ! !} \sum_{p=0}^{q-1} \frac{(2 p-1) ! !}{(2 p) ! !}\left(\frac{(2 p+1) ! !}{(2 p+4) ! !}\right)
$$

Da Afirmação (B.4.1) e de (B.15), temos que

$$
B_{2}^{2 q}=+\frac{8 q \pi}{3}\left(\frac{(2 q-1) ! !}{(2 q+2) ! !}\right)
$$


APÊNDICE B 


\section{Referências Bibliográficas}

[BNdOC88] Angelo Barone-Netto e Mauro de Oliveira Cesar. Non-conservative positional systems stability. Dynamics and Stability of Systems, 2(3-4):213-221, 1988. 11, 13, 14, 18, $19,20,72$

[BNdOC91] Angelo Barone-Netto e Mauro de Oliveira Cesar. The existence of liapunov functions for some non-conservative positional mechanical systems. Journal of Differential Equations, 91(2):235-244, 1991. 15, 16, 20

[BNdOC92] Angelo Barone-Netto e Mauro de Oliveira Cesar. A necessary and sufficient condition for the stability of the equilibrium. Journal of Differential Equations, 96(2):142 151, 1992. 15, 17

[BNdOC05] Angelo Barone-Netto e Mauro de Oliveira Cesar. Some central forces-stability. Qualitative theory of Dynamical Systems, 6:1-8, 2005. 2

[BNdOCG02] Angelo Barone-Netto, Mauro de Oliveira Cesar e Gianluca Gorni. A computational method for the stability of a class of mechanical systems. Journal of Differential Equations, 184(1):1 - 19, 2002. 2, 4, 15, 17, 19, 20, 30, 32, 76

[BNZ86] Angelo Barone-Netto e Gaetano Zampieri. Atractive central forces may yield liapunov instability. Dynamical Systems and Partial Differential Equations, Proceedings of the rth ELAM, Editorial Equinoccio (Caracas), páginas 105-112, 1986. 11, 13, 15, 72, 75

[dS02] Emivan Ferreira da Silva. A função período para uma classe de sistemas hamiltonianos. Tese de Doutorado, Instituto de Matemática e Estatística, Universidade de São Paulo, Brasil, Novembro 2002. 9, 11

[MS66] W. Magnus e S.Wilker. Hill's Equations. Interscience, 1966. 5, 12, 15, 16, 18

[MSR04] M.W.Hirsh, S.Smale e R.L.Devaney. Differential Equations, Dynamical Systems, and a Introduction to Chaos. Elsevier,USA, 2004. 5

[Sai11] Marcelo José Saia. Uma introdução à Teoria de Singularidades. Notas Didáticas,ICMC-USP, 2011. 5

[Wei] Eric W Weisstein. Cosine Integral. From MathWorld-A Wolfram Web Resource. https://mathworld.wolfram.com/CosineIntegral.html. Acesso em 10 de nov. 2020. 46

[Zam88] Gaetano Zampieri. Liapunov stability for some central forces. Journal of Differential Equations, 74(2):254-265, 1988. 2, 4, 9, 11, 15, 72

[Zam89] Gaetano Zampieri. Solving a collection of free coexistence-like problems in stability. Rendiconti del Seminario Matematico dell'Università di Padova, 81:95-106, 1989. 17, 18, 81 
[Zam99] Gaetano Zampieri. The last free coexistence-like problem. An International Jounal for Theory \& Applications, 3:933-943, 1999. 17, 18

[Zam11] Gaetano Zampieri. Completely integrable hamiltonian systems with weak lyapunov instability or isochrony. Communications in Mathematical Physics, 303:73 - 87, 2011. $9,15,19,22,23,24,81$ 